Dimas Betioli Ribeiro

\title{
ANÁLISE DA INTERAC̄̃̃O SOLO-ESTRUTURA VIA ACOPLAMENTO MEC-MEF
}

Dissertação apresentada à Escola de Engenharia de São Carlos da Universidade de São Paulo, como parte dos requisitos para a obtenção do Título de Mestre em Engenharia de Estruturas.

Orientador : Professor Doutor João Batista de Paiva

São Carlos 


\section{Agradecimentos}

Agradeço primeiramente ao meu orientador João Batista de Paiva por toda a atenção e paciência e também à indispensável ajuda do Valério, sem a qual não teria sido possível concluir este trabalho. Também aos professores Nelson Aoki, Libânio Miranda Pinheiro e José Samuel Giongo pela assistência.

Agradeço também à minha namorada, Ana Carolina Lorena, por toda a ajuda para com o Latex e também na correção do texto da dissertação.

À minha família por toda compreensão e apoio.

À ajuda de vários dos meus colegas do departamento, os velhos e novos amigos, que me auxiliaram em diversos detalhes referentes ao trabalho. São tantos que nem cabem aqui.

Por fim, agradeço ao pessoal da secretaria do SET por toda a força de vontade e atenção. 


\section{Resumo}

RIBEIRO, D. B. (2005). Análise da Interação Solo-Estrutura via Acoplamento MEC-MEF. 121p. Dissertação (Mestrado) - Escola de Engenharia de São Carlos, Universidade de São Paulo, São Carlos, 2005.

O objetivo central deste trabalho é o estudo da interação do solo com a estrutura. Para tanto, são introduzidos mais recursos na ferramenta numérica desenvolvida no trabalho de ALMEIDA (2003a). O solo é modelado pelo Método dos Elementos de Contorno (MEC) tridimensional, aplicando a solução fundamental de Kelvin. É possível analisar problemas nos quais o solo é composto por camadas de diferentes características físicas, apoiadas em uma superfície de deslocamento nulo e enrijecidas por elementos de fundação, também modelados pelo MEC tridimensional. A superestrutura tridimensional, diferentemente do modelo utilizado em ALMEIDA (2003a), é simulada pelo Método dos Elementos Finitos (MEF), sendo composta por elementos planos e reticulares com seis graus de liberdade por nó. Também é introduzido no programa o recurso de simular um número qualquer de blocos, modelados pelo MEC tridimensional, apoiados sobre o solo. Estes blocos podem ser utilizados como elementos de fundação para o edifício, permitindo estudar a interação do solo em conjunto com os blocos e o edifício. São analisados alguns exemplos, nos quais é validada a formulação empregada e é demonstrada a necessidade de se considerar a interação do solo com a estrutura em problemas práticos de engenharia.

Palavras-chave: interação solo/estrutura; método dos elementos de contorno; solo não-homogêneo; acoplamento MEC/MEF; bloco; edifício. 


\section{Abstract}

RIBEIRO, D. B. (2005). Analysis of soil-structure interaction using BEM-FEM coupling. 121p. M.Sc. Dissertation - Escola de Engenharia de São Carlos, Universidade de São Paulo, São Carlos, 2005.

The main objective of this work is to study the soil structure interaction problem. For such, more resources in the numerical tool developed in ALMEIDA (2003a) are introduced. The soil is simulated by the three-dimensional Boundary Element Method (BEM), applying Kelvin's fundamental solution. It is possible to analyze problems in which the soil is composed by layers of different physical characteristics, supported by a rigid and adhesive interface and reinforced by foundation elements, also simulated by the three-dimensional BEM. The three-dimensional superstructure is simulated using the Finite Element Method (FEM), with shell and frame elements with six degrees of freedom by node. This model is different of the one used in ALMEIDA (2003a). It is also introduced in the program the resource to consider blocks, simulated by the three-dimensional BEM and supported by the soil. These blocks can be used as foundation elements for the building, coupling the non-homogeneous soil-foundation-blocks-superstructure system as a whole. Some examples are analyzed, in order to validate the theory employed and demonstrate the necessity of considering the soil structure interaction in practical problems of engineering.

Key-words: soil-structure interaction; boundary element method; non-homogeneous soil; coupling BEM-FEM; block; building. 


\section{Sumário}

Agradecimentos ii

Resumo iii

Abstract iv

Sumário $\quad$ v

$\begin{array}{lll}1 & \text { Introdução } & 1\end{array}$

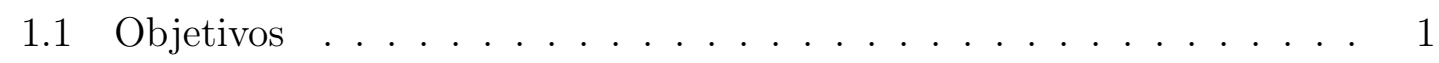

1.2 Revisão bibliográfica . . . . . . . . . . . . . . . . . . . . . . . . . . . . . . . . . . . . .

1.3 Organização da dissertação . . . . . . . . . . . . . . . . . . . 9 9

2 O Método dos Elementos de Contorno 10

2.1 Introdução . . . . . . . . . . . . . . . . . . . . . . . 10

2.2 Equacionamento básico do problema elástico linear . . . . . . . . . . 11

2.2 .1 O estado de tensão . . . . . . . . . . . . . . . . . . . . 11

2.2 .2 O estado de deformação . . . . . . . . . . . . . . . . . 14

2.2 .3 Relações constitutivas . . . . . . . . . . . . . . . . . . . . 15

2.3 Soluções fundamentais . . . . . . . . . . . . . . . . . . 16

2.4 Equações integrais de contorno $\quad \ldots \ldots \ldots$

$2.4 .1 \quad$ Equação integral para pontos do contorno . . . . . . . . . . 22

2.4 .2 Formulação de elementos de contorno . . . . . . . . . . . . . . 24

2.4 .3 Transformação de coordenadas . . . . . . . . . . . . . . . . . . 28

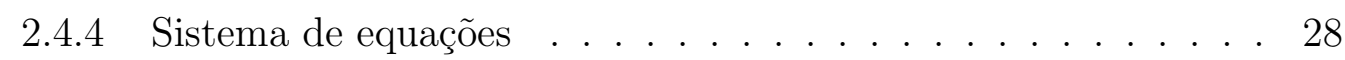

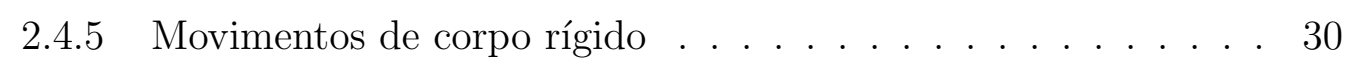

2.4 .6 Pontos internos . . . . . . . . . . . . . . . . . . 32

2.4 .7 Subelementação . . . . . . . . . . . . . . . . . . 33

2.5 Considerações finais $\ldots \ldots \ldots$. . . . . . . . . . . . . . . . . . . 34

3 O Método dos Elementos Finitos 36

3.1 Introdução . . . . . . . . . . . . . . . . . . . 36 
$3.2 \quad$ O Princípio dos Trabalhos Virtuais (PTV) . . . . . . . . . . . . 36

3.3 Elementos finitos laminares $\ldots \ldots$. . . . . . . . . . . . . . 40

3.4 Graus de liberdade do elemento finito laminar . . . . . . . . . . . . 43

3.5 Rotação de eixos . . . . . . . . . . . . . . . . . . . . . . 45

3.6 Elementos utilizados no edifício $\ldots \ldots \ldots$. . . . . . . . . . . . 46

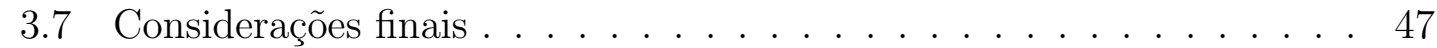

4 O Método da Rigidez Sucessiva 48

4.1 Introdução . . . . . . . . . . . . . . . . . . . . . . . . . . . . 48

$4.2 \quad$ O MRS aplicado ao solo estratificado . . . . . . . . . . . . . . . . . 49

$4.3 \quad$ O MRS aplicado ao solo com estacas . . . . . . . . . . . . . 56

4.4 Considerações finais . . . . . . . . . . . . . . . . . . . . 66

$\begin{array}{lll}5 & \text { Interação do solo com estrutura } & 68\end{array}$

$5.1 \quad$ Introdução . . . . . . . . . . . . . . . . . . . . . . . 68

5.2 Acoplamento entre o solo e um bloco . . . . . . . . . . . . . . 68

5.3 Acoplamento entre o solo e $N$ blocos $\ldots \ldots \ldots \ldots$. . . . . . . 72

5.4 Acoplamento entre o solo e o edifício . . . . . . . . . . . . . . 77

5.5 Acoplamento entre solo, blocos e edifício . . . . . . . . . . . . . 81

5.6 Considerações finais $\ldots \ldots \ldots$. . . . . . . . . . . . . . . . . . . . 83

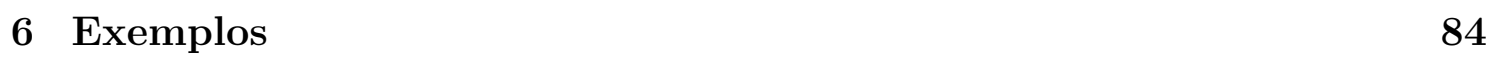

6.1 Introdução . . . . . . . . . . . . . . . . . . . . . . 84

6.2 Bloco sem estacas . . . . . . . . . . . . . . . . . . . . . . 84

6.3 Bloco sobre uma estaca . . . . . . . . . . . . . . . . . . . . . . . . 89

6.4 Interação entre dois blocos . . . . . . . . . . . . . . . . . . . . 95

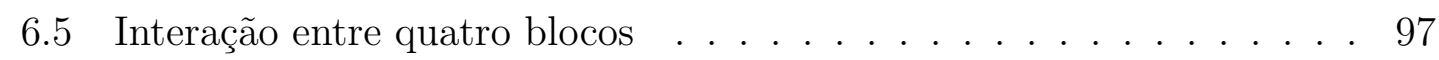

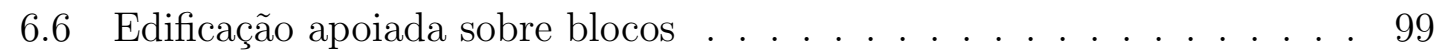

6.7 Considerações finais . . . . . . . . . . . . . . . . . 106

\begin{tabular}{lll}
\hline 7 & Conclusões & 107
\end{tabular}

7.1 Observações finais . . . . . . . . . . . . . . . . . . . . . . . . . . 107

7.2 Propostas para trabalhos futuros . . . . . . . . . . . . . . 109

\begin{tabular}{lr}
\hline Referências Bibliográficas & 111
\end{tabular}

\begin{tabular}{|lr}
\hline A Integral singular & 119
\end{tabular} 


\section{Lista de Figuras}

2.1 Sólido tridimensional . . . . . . . . . . . . . . . . . . . . . . . 11

2.2 Equilíbrio em um elemento infinitesimal $\ldots \ldots \ldots \ldots \ldots$

$2.3 \quad$ Equilíbrio em um elemento infinitesimal $\ldots \ldots \ldots \ldots \ldots$

$2.4 \quad$ Equilíbrio de um tetraedro . . . . . . . . . . . . . . . . 13

2.5 Condições de contorno arbitrárias . . . . . . . . . . . . . . . 19

$2.6 \quad$ Ponto $i$ envolvido por uma semi-esfera de raio $\varepsilon \ldots \ldots \ldots \ldots . \ldots 22$

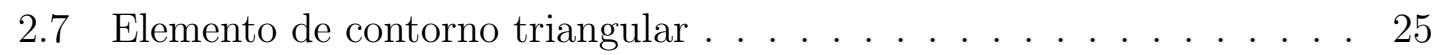

2.8 Funções interpoladoras adotadas . . . . . . . . . . . . . . . . . 26

2.9 Sistemas de coordenadas global e local . . . . . . . . . . . . 26

2.10 Subelementação convencional . . . . . . . . . . . . . . 34

2.11 Subelementação progressiva . . . . . . . . . . . . . . . . . 34

3.1 Sólido qualquer com condições de contorno arbitrárias . . . . . . . . 37

3.2 Direção dos deslocamentos e posição dos pontos $P$ e $P$. . . . . . . . 41

3.3 Graus de liberdade do elemento finito de membrana $\mathrm{FF}$. . . . . . . . 43

3.4 Graus de liberdade do elemento finito DKT . . . . . . . . . . . . 44

3.5 Graus de liberdade do elemento finito laminar DKT/FF . . . . . . . 44

$3.6 \quad$ Sistema de coordenadas global $x_{1} x_{2} x_{3}$ e local $x_{1}^{l} x_{2}^{l} x_{3}^{l} \ldots \ldots \ldots .45$

4.1 Solo estratificado em camadas . . . . . . . . . . . . . . . . . 50

4.2 Camada $i$ isolada . . . . . . . . . . . . . . . . . . . . . 50

$4.3 \quad$ Equilíbrio e compatibilidade entre as camadas 1 e $2 \ldots \ldots \ldots 5$

4.4 Ordem de obtenção das matrizes no processo iterativo . . . . . . . . . 54

4.5 Ordem do cálculo dos valores de contorno no processo iterativo. . . . 56

4.6 Contorno da camada $i$ dividido em trechos $\ldots \ldots \ldots \ldots \ldots$

4.7 Caso mais geral para uma camada $i \ldots \ldots \ldots \ldots \ldots$

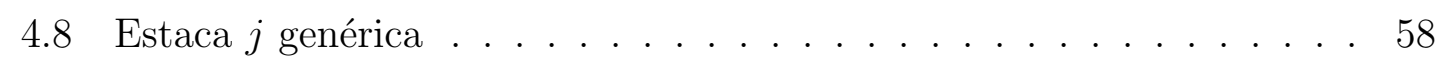

5.1 Conjunto formado pelo solo e o bloco . . . . . . . . . . . . . . 69

5.2 Interação do solo com o edifício . . . . . . . . . . . . . . . 77

5.3 Incompatibilidade entre as cargas de superfície e as cargas nodais . . 78

$5.4 \quad$ Aproximações lineares adotadas para $w \mathrm{e} p \ldots \ldots \ldots \ldots$ 
5.5 Graus de liberdade do MEF e do MEC . . . . . . . . . . . . . . . . 81

5.6 Conjunto formado pelo solo, blocos e edifício . . . . . . . . . . . . . 82

5.7 Casca flexível conectada ao topo do bloco . . . . . . . . . . . . . . . . 82

$6.1 \quad$ Vista em planta . . . . . . . . . . . . . . . . . . . 84

6.2 Vista em perfil . . . . . . . . . . . . . . . . . . . 84

6.3 Vista em planta dos deslocamentos verticais . . . . . . . . . . . 85

6.4 Deslocamentos no contato . . . . . . . . . . . . . . . 86

6.5 Tensões de contato . . . . . . . . . . . . . . . . . . . . 87

6.6 Tensões de contato . . . . . . . . . . . . . . . . . . . . . 87

6.7 Superfície de deslocamentos nulos . . . . . . . . . . . . . . . . 88

6.8 Deslocamentos verticais em planta considerando a superfície de deslocamentos nulos . . . . . . . . . . . . . . . . . . . 88

6.9 Comparação dos deslocamentos no contato . . . . . . . . . . . . . . 89

6.10 Vista em perfil . . . . . . . . . . . . . . . . 90

6.11 Vista em planta . . . . . . . . . . . . . . . . . . . . . 90

6.12 Vista em planta dos deslocamentos verticais . . . . . . . . . . . . 90

6.13 Deslocamentos no contato . . . . . . . . . . . . . . . . . . 91

6.14 Vista em planta das tensões de contato . . . . . . . . . . . . . . . . . 91

6.15 Tensões no contato . . . . . . . . . . . . . . . . . . . 92

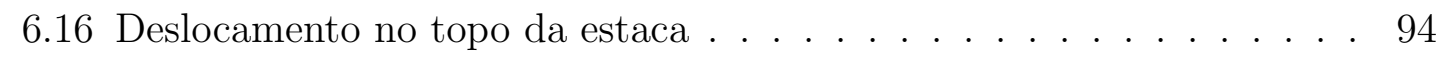

6.17 Vista em planta . . . . . . . . . . . . . . . . . . . . 95

6.18 Vista em perfil . . . . . . . . . . . . . . . . . . 95

6.19 Vista em planta dos deslocamentos verticais . . . . . . . . . . . . 96

6.20 Deslocamento vertical na base dos blocos . . . . . . . . . . . . . . 96

6.21 Tensão de contato na base dos blocos . . . . . . . . . . . . . . . . . . 97

6.22 Vista em planta . . . . . . . . . . . . . . . . . . 97

6.23 Vista em planta dos deslocamentos verticais . . . . . . . . . . . 98

6.24 Deslocamento vertical na base dos blocos . . . . . . . . . . . . . . . . 99

6.25 Tensão de contato na base dos blocos . . . . . . . . . . . . . . . . . . 99

6.26 Edificação tridimensional em perspectiva . . . . . . . . . . . . . 100

6.27 Edificação tridimensional em perfil . . . . . . . . . . . . . . . 100

6.28 Vista em planta dos blocos . . . . . . . . . . . . . . . . . 100

6.29 Seção transversal dos pilares e vigas . . . . . . . . . . . . . . . . . . 101

6.30 Geometria da laje . . . . . . . . . . . . . . . . . . . 101

6.31 Vista em planta dos deslocamentos verticais . . . . . . . . . . . . . 102

6.32 Deslocamento vertical na base dos blocos . . . . . . . . . . . . . . . . 103

6.33 Tensão de contato na base dos blocos . . . . . . . . . . . . . . . . 103

6.34 Deslocamento vertical no eixo $s_{1} \ldots \ldots$. . . . . . . . . . . 104 
6.35 Deslocamento vertical no eixo $s_{2} \ldots \ldots$. . . . . . . . . . 104

6.36 Momento MX no eixo $s_{1} \ldots \ldots \ldots$. . . . . . . . . . . . . 105

6.37 Momento MY no eixo $s_{1} \ldots \ldots$. . . . . . . . . . . . 105

6.38 Deslocamento horizontal do pilar . . . . . . . . . . . . . 106

A.1 Sistema de coordenadas esféricas . . . . . . . . . . . . . . . 120 


\section{Capítulo 1}

\section{Introdução}

\section{$1.1 \quad$ Objetivos}

O objetivo deste trabalho é alterar o programa desenvolvido em ALMEIDA (2003a) para analisar um conjunto composto por um solo estratificado e blocos, ambos modelados pelo MEC em três dimensões. A parcela referente às camadas de solo pode ser aproveitada do programa computacional desenvolvido em ALMEIDA (2003a), sendo necessário então implementar o acoplamento entre blocos tridimensionais e este conjunto. Para isto, cada bloco é considerado uma nova subregião e acoplado ao sistema final de equações regentes do problema.

O ojetivo final, finalizando a parte de implementação, é acoplar ao conjunto formado por solo e blocos um edifício tridimensional modelado pelo MEF.

\subsection{Revisão bibliográfica}

A interação do solo com a estrutura representa um sistema mecânico integrado. Este mecanismo, no entanto, geralmente é estudado separando-se a parte estrutural da parte geotécnica. Esta simplificação advém do alto grau de complexidade envolvido em se avaliar este fenômeno mecânico. Cada um dos subsistemas isolado leva a um vasto campo de estudo, tanto na variabilidade de parâmetros físicos e geométricos como nas correspondentes idealizações dos modelos mecânicos. Somase a isto o fato de que cada pesquisador tende a focar mais sua própria área de conhecimento em seus estudos.

O engenheiro geotécnico, ao analisar a integração entre a subestrutura e o maciço de solos, geralmente não considera mudanças de configuração que possam ocorrer na superestrutura. Estas mudanças podem levar a um estado de tensões não previsto no sistema formado pela subestrutura e o maciço. Em contrapartida, o engenheiro estrutural, por estar voltado aos fenômenos que ocorrem na superestrutura, 
dificilmente leva em conta os efeitos que ocorrem no solo devido à absorção das ações. Estes efeitos causam modificações na superestrutura que nem sempre são desprezíveis.

Além disto, um fenômeno comum que ambos os pesquisadores usualmente não enfatizam é o efeito de grupo que altera o estado de tensões do sistema envolvido. Assim, além de se avaliar o sistema formado pela superestrutura, subestrutura e maciço de solos de forma não acoplada, considera-se este sistema livre do efeito de vizinhança. Isto pode levar a estados de deformações e tensões diferentes dos previstos nos projetos envolvidos.

Como a análise da interação do solo com a estrutura de forma integrada é complexa, é comum encontrar na literatura trabalhos que considerem estes sub-sistemas de forma separada. Em se tratando da superestrutura isolada, diversos trabalhos podem ser citados.

Em BATOZ (1980) são estudadas placas fletidas com o emprego do Método dos Elementos Finitos (MEF), mais precisamente com o elemento DKT. Também empregam o MEF em análise de estruturas os trabalhos de BATHE (1982) e ONATE (1995). Em BEZERRA (1995) utiliza-se o MEF no cálculo de edifícios, enquanto em RIOS (1991) analisa-se a envoltória de esforços em edifícios altos.

Como trabalhos mais recentes, pode-se citar PELETEIRO (1996), MESQUITA (1998) e DUARTE, BABUSCA \& ODEN (2000). O primeiro utiliza a teoria do MEF na descrição de um elemento de membrana com graus de liberdade rotacionais nos nós. O segundo analisa estruturas com elementos de casca e o terceiro aplica o MEF tridimensional em problemas de mecânica estrutural.

Considerando os trabalhos que envolvem interação do solo com a estrutura, é possível distinguir alguns modelos nos quais é baseada a simulação do solo.

O modelo de WINKLER (1867) consiste na substituição do solo por um conjunto de molas discretas. Os coeficientes das molas são adotados segundo o problema que se está analisando. Geralmente são utilizadas correlações empíricas na determinação destes coeficientes, o que pode prejudicar a precisão dos resultados. Pode-se considerar uma estaca imersa no solo, modelando-a como um elemento de viga ou uma barra rígida. Entretanto, caso se queira analisar uma estaca de comprimento genérico imersa em um solo qualquer, os resultados perdem precisão devido à dificuldade de se determinar os coeficientes das molas. A maior vantagem do modelo de Winkler é sua simplicidade e consequente facilidade de implementação, podendo-se citar os trabalhos de CHEUNG \& ZIENKIEWICZ (1965), RANDOLPH \& WROTH (1979) e WITT (1984). Também relacionados a este tema os trabalhos de LEE (1993) e MYLONAKIS \& GAZETAS (1998) analisam grupos de estacas, calculando recalques e tensões e considerando o solo não-homogêneo.

Outra técnica que pode ser encontrada na literatura, baseada na teoria da elas- 
ticidade, parte do manuseio de equações diferenciais e integrais com o intuito de encontrar soluções analíticas ou semi-analíticas para problemas específicos de interação do solo com a estrutura. O solo é geralmente assumido como elástico, linear e homogêneo, simplificando assim o equacionamento. A principal desvantagem desta técnica é que ela se restringe a um pequeno grupo de problemas, não podendo ser aplicada em casos gerais. Relacionado a este contexto, pode-se citar o trabalho de BURMISTER (1945a), no qual é analisado um solo formado por duas camadas, sendo a inferior um meio semi-infinito. A carga externa, aplicada na superfície livre do solo, é uma força concentrada. Partindo do equacionamento da teoria da elasticidade, são obtidas soluções semi-analíticas de deslocamento e tensão no domínio das camadas. Em BURMISTER (1945b), esta análise é extendida para três camadas de solo.

As soluções de Burmister foram utilizadas por outros autores. Em CHAN, KARASUDHI \& LEE (1974) é feito um estudo para um solo composto por duas camadas, aplicando uma força concentrada horizontal ou vertical no interior destes domínios. O ponto de partida são as equações apresentadas por BURMISTER (1945a), chegando então a um procedimento mais abrangente. Outro autor que aplicou as soluções de Burmister foi POULOS (1967), com o intuito de obter fatores de influência do solo para diferentes carregamentos aplicados em sua superfície.

Para melhorar os resultados obtidos em CHAN, KARASUDHI \& LEE (1974), DAVIES \& BANERJEE (1978) aplicam métodos de quadratura, tornando o cálculo das integrais mais preciso. As funções de deslocamento são obtidas para várias combinações de parâmetros elásticos. Em casos simples, esta abordagem leva a bons resultados e com alta convergência. Nos casos mais complexos, entretanto, esta técnica se torna muito trabalhosa e se faz necessária a utilização de soluções numéricas. Em casos que envolvam meios não-homogêneos e grupos de estacas, por exemplo, as integrais se tornam muito complexas e a precisão dos resultados fica prejudicada.

Um modelo conhecido que pode ser aplicado na simulação do solo é o Método da Camada Finita (MCF). Neste método, o Método dos Elementos Finitos (MEF) é combinado à técnica da transformada de Fourier. Aplicando esta teoria em um problema tridimensional este fica reduzido a apenas duas dimensões, o que torna simples sua implementação. Esta ferramenta é eficiente em problemas elásticos, podendo o solo ser formado por camadas de diferentes propriedades físicas e anisótropo. É possível também considerar estacas imersas no solo, bastando considerar condições de compatibilidade de deslocamentos e equilíbrio de forças na superfície de contato entre o solo e o fuste das estacas. Uma falha que pode ser apontada no MCF é que esta ferramenta pode ser aplicada somente em problemas de domínio elástico. Alguns trabalhos relacionados ao MCF que podem ser citados são SMALL \& 
BOOKER (1984), BOOKER, CARTER \& SMALL (1989), LEE \& SMALL (1991), SOUTHCOTT \& SMALL (1996) e TA \& SMALL (1998). Ainda relacionado a este tema pode ser citado o trabalho de CHEUNG, THAM \& GUO (1988), no qual é apresentada uma variação do MCF denominada Método da Camada Infinita (MCI).

Outra técnica numérica, amplamente utilizada em problemas de engenharia, é o Método dos Elementos Finitos (MEF). Esta ferramenta é extremamente versátil e, na maioria dos casos, é a opção mais eficiente e prática para a análise de estruturas. No entanto, as vantagens do MEF são poucas quando se quer analisar situações de domínio infinito, que constitui o caso de problemas de interação do solo com a estrutura. Isto acontece porque o MEF é um método de domínio, sendo necessário dividir o domínio do problema em elementos. Para simular um domínio semi-infinito se torna necessário aplicar as condições de contorno do problema a grandes distâncias, resultando em um grande número de elementos, nós e, consequentemente, equações a serem resolvidas. Além disto, o armazenamento de informações tais como coordenadas de nós e conectividades entre nós e elementos é onerosa. Estes problemas se tornam acentuados principalmente em análises tridimensionais. Por estes motivos são escassos na literatura trabalhos que abordem a interação do solo com a estrutura simulando o solo pelo MEF, podendo-se citar OTTAVIANI (1975). Em CHOW \& TEH (1991), o MEF é aplicado no problema de uma placa rígida com estacas apoiada em um solo elástico, linear e finito, estando a placa em contato com o solo. O módulo de elasticidade do solo foi adotado variando linearmente com a profundidade.

Uma ferramenta numérica que pode ser considerada eficiente para modelar o solo em problemas de interação do solo com a estrutura é o Método dos Elementos de Contorno (MEC). Como somente o contorno do domínio do problema é dividido em elementos, a análise fica reduzida em uma dimensão. Isto diminui o custo computacional envolvido na resolução de equações, além de simplificar o armazenamento de dados. Devido a estas vantagens vários autores utilizam o MEC na análise da interação do solo com a estrutura, conforme pode ser observado nos trabalhos citados a seguir.

No trabalho de MINDLIN (1936), foram obtidas soluções fundamentais em deslocamento e força para uma força concentrada unitária aplicada no interior de um meio semi-infinito homogêneo, elástico, linear e isotrópico. Como estas soluções podem ser empregadas no equacionamento do MEC, vários pesquisadores analisam problemas de semi-espaço infinito com estas soluções.

No trabalho de POULOS (1967) é empregado o modelo de STEINBRENNER (1934), utilizando as soluções de Mindlin para calcular valores de deslocamento no interior de um solo apoiado em uma superfície de deslocamento nulo. Neste modelo, escolhe-se a profundidade na qual se encontra a superfície de deslocamento 
nulo e calcula-se, por Mindlin, o deslocamento de um ponto pertencente a esta superfície. Em seguida determina-se, também por Mindlin, o deslocamento em um ponto qualquer da camada de solo. O deslocamento neste ponto é obtido então pela diferença entre o valor calculado no ponto e o valor calculado na superfície de deslocamento nulo.

O modelo de Steinbrenner foi aplicado novamente em POULOS \& DAVIES (1968), considerando agora uma estaca incompressível imersa no solo. Submetida a uma carga axial, esta estaca é dividida em elementos cilíndricos, cada qual submetido a uma tensão de cisalhamento uniforme. A ponta da estaca é uma base alargada, na qual se considera unicamente a tensão axial.

Esta mesma formulação foi empregada em POULOS (1968), considerando então grupos de estacas. O ponto de partida é a interação de duas estacas, a partir da qual é obtido um coeficiente de influência $\alpha$. Para grupos com mais de duas estacas é feita uma superposição de efeitos, tomando as estacas duas a duas. São analisados grupos simétricos e gerais, sendo as estacas idênticas e estando submetidas ao mesmo carregamento.

Esta abordagem foi utilizada também em MATTES \& POULOS (1969), considerando as estacas compressíveis na direção vertical. São calculados deslocamentos verticais utilizando a técnica das diferenças finitas no cálculo de uma equação diferencial. Estes deslocamentos são obtidos após a determinação das tensões de cisalhamento ao longo da estaca.

Foi adicionado, em POULOS (1971a), o recurso de aplicar cargas horizontais e momentos no topo de uma estaca isolada. Este procedimento foi então estendido para grupos de estacas em POULOS (1971b).

Em BUTTERFIELD \& BANERJEE (1971), são analisados grupos de estacas ligadas por uma placa rígida. É aplicada uma força concentrada e vertical na placa, determinando então o deslocamento vertical estabelecido no sistema. Em BANERJEE (1976) é feito um estudo semelhante, considerando então estacas inclinadas. É utilizado, neste trabalho, o método indireto das equações integrais. A carga aplicada na placa pode ser uma força vertical, horizontal ou um momento. Outra extensão foi adicionada a este trabalho em BANERJEE (1978), tornando possível simular um solo com módulo de elasticidade linearmente variável com a profundidade.

Em MAIER \& NOVATI (1987), foi desenvolvida uma técnica baseada no MEC para a análise de solos estratificados denominada de "Método da Rigidez Sucessiva" (MRS). Tratando cada estrato como uma sub-região e utilizando condições de equilíbrio e compatibilidade existentes entre estratos adjacentes, é possível transferir a rigidez da camada inferior para sua adjacente superior. Desta forma, chega-se à camada da superfície com uma matriz na qual estão incorporadas as influências de todas as outras camadas inferiores. No trabalho de MAIER \& NOVATI (1987) este 
procedimento é aplicado em exemplos bidimensionais. Na conclusão chega-se a uma matriz final que não é mal-condicionada e cuja resolução demanda um custo computacional inferior ao requerido pela técnica convencional de sub-regiões do MEC. Este método pode ser aplicado na introdução de outras sub-regiões, como em análise de túneis ou de elementos de fundação.

Podem ser encontrados também, na literatura, trabalhos que envolvam sólidos elásticos tridimensionais modelados pelo MEC. Estes estudos utilizam as soluções fundamentais de Kelvin, descritas em LOVE (1944). Em NAKAGUMA (1979) esta teoria foi empregada em conjunto com as soluções fundamentais de MINDLIN (1936) e BOUSSINESQ (1885) no estudo de um meio semi-infinito. São apresentados exemplos relacionados à interação do solo com estrutura. O método dispensa a divisão da superfície livre em elementos, o que torna a ferramenta versátil. Relacionados à solução fundamental de Kelvin, também podem ser citados BANERJEE (1976) e BANERJEE \& DAVIES (1977). Estes autores apresentam uma ferramenta para a análise de estacas conectadas ou não por uma placa rígida e imersas em um meio heterogêneo.

Com o intuito de aumentar a abrangência de seus trabalhos, alguns autores estudam o acoplamento de diferentes formulações. Os trabalhos de BREBBIA \& GEORGIOU (1979) e BEER \& MEEK (1981) podem ser citados neste contexto por utilizarem uma combinação do MEC com o MEF na análise de problemas relacionados à geomecânica. O trabalho de MEEK (1988) também utiliza uma combinação entre o MEC e o MEF no estudo de problemas elásticos tridimensionais.

Existem ainda técnicas para simulação do solo que não podem ser enquadradas nas descritas anteriormente, podendo-se citar os trabalhos de HETENYI (1950), onde é definida a fundação de Hetenyi, WANG,IANG \& WANG (2001), que emprega a fundação de Pasternak, KERR (1964), que utiliza a fundação de Kerr e KERR (1965), que emprega a fundação generalizada. Todos estes métodos partem do modelo de Winkler em conjunto com modelos contínuos. São introduzidos elementos estrututrais para conectar as molas discretas ou então são adotadas simplificações para os modelos contínuos. Estas simplificações são ajustadas por valores reais de deslocamento ou tensão.

No trabalho de CHIN \& CHOW (1990) o MEC é empregado na análise de grupos de estacas, porém a solução fundamental utilizada na formulação é obtida a partir de CHAN, KARASUDHI \& LEE (1974). Esta solução corresponde a uma força concentrada horizontal ou vertical aplicada no interior de um solo composto por duas camadas.

Em FRASER \& WARDLE (1976), é apresentada uma interessante técnica derivada do MEF. Nesta ferramenta, utilizada na análise da interação de uma placa flexível com o solo, somente a porção carregada do solo necessita ser dividida em 
elementos. O método é intitulado aproximação por elemento de superfície, e se baseia em funções ponderadoras de meio semi infinito. A placa flexível é abordada pelo MEF convencional.

Em PAN (1997) é analisado um solo formado por diferentes camadas isotrópicas. Para tornar mais eficiente a implementação da interação das camadas, modeladas pelo MEC, são utilizadas as funções de Green. Uma falha desta ferramenta é a impossibilidade de se considerar elementos de fundação, uma vez que o método permite unicamente a estratificação horizontal das camadas.

São estudadas em SADECKA (2000) placas grossas apoiadas sobre o solo. Este admite camadas de diferentes propriedades além de uma superfície indeslocável localizada a uma distância prescrita. Utiliza-se o MEF na placa e o modelo de Kolar-Nemec no solo, acoplando as formulações posteriormente. Para representar a superfície do meio semi-infinito, foram utilizados elementos infinitos. Apesar de permitir placas grossas e solos estratificados a abordagem é limitada somente a estas estruturas, impossibilitando a análise de sistemas mais complexos.

Entre os pesquisadores brasileiros, pode-se citar vários trabalhos relacionados à interação do solo com estrutura. O trabalho de AOKI \& LOPES (1975) faz um estudo das fundações profundas, determinando deslocamentos e tensões cisalhantes de forma semelhante ao trabalho de POULOS (1968). Em REIS (2000) esta teoria é empregada novamente, agora no estudo de fundações rasas.

O trabalho de GUSMÃO (1990) também utiliza a formulação de AOKI \& LOPES (1975). São analisados deslocamentos em um edifício bidimensional, apoiado em um solo estratificado que se encontra sobre um superfície de deslocamento nulo.

Em MOURA (1995) é analisada a interação de um edifício tridimensional com o solo. O edifício, modelado pelo MEF, é composto por elementos reticulares que representam os pilares e vigas e por diafragmas rígidos para simular as lajes. O solo, considerado uma camada homogênea apoiada em uma superfície de deslocamento nulo, é modelado segundo o procedimento apresentado em AOKI \& LOPES (1975). A conecção dos pilares do eficício com o solo é feita por meio de elementos de sapata.

No trabalho de HOLANDA (1998), é empregado o procedimento descrito em POULOS (1967) para simular uma camada de solo homogêneo apoiada em uma superfície de deslocamento nulo. A estrutura considerada é um edifício de concreto armado apoiado em fundações diretas.

Em FERRO (1998) é analisada a interação de estacas modeladas pelo MEF e um solo simulado pelo MEC em abordagem tridimensional. Também é feita a interação de uma placa com o solo, sendo a placa modelada pelo MEF com o elemento DKT. O solo é homogêneo e infinito, e pode ser analisado com comportamento não linear.

Em ANTUNES \& IWAMOTO (2000), estuda-se a interação de estacas com um solo estratificado. O solo, apoiado em uma camada de deslocamento nulo, é mode- 
lado utilizando a teoria descrita em POULOS (1967). São aplicados incrementos de carga na estaca, e sua ponta absorve carga somente após a mobilização de todo o fuste.

Em MESQUITA \& CODA (2000) é estudado um pórtico plano apoiado sobre uma camada de solo finita. Nesta análise visco-elástica, o solo é modelado pelo MEC em abordagem bidimensional e a conecção deste com o pórtico é feita por meio de sapatas, sendo possível apoiar cada uma em um meio com propriedades diferentes. Em LEITE, CODA \& VENTURINI (2001) é utilizada esta mesma teoria, incluindo elementos enrigecedores no solo representando estacas.

No trabalho de MENDONÇA \& PAIVA (2003) são analisados grupos de estacas, que podem estar conectadas por uma placa flexível. O solo é considerado um semiespaço infinito elástico, linear e homogêneo, representado por equações integrais utilizando a solução fundamental de Mindlin. Cada estaca é representada por um único elemento finito reticular com três nós, e a força vertical cisalhante ao longo do fuste é aproximada por uma função quadrática. Na extremidade inferior da estaca a tensão é considerada constante ao longo da seção transversal, e um dos nós se localiza nesta extremidade. A placa é modelada pelo MEF utilizando dois tipos de elementos finitos planos, o DKT e o HSM. Em MENDONÇA \& PAIVA (2000) é feita uma análise semelhante, mas modelando a placa flexível também por equações integrais ao invés de elementos finitos.

Em ALMEIDA (2003a) é proposta uma formulação para a análise da interação do solo com a estrutura na qual o solo, composto por uma ou mais camadas apoiadas em uma superfície de deslocamento nulo, é modelado pelo MEC em abordagem tridimensional. Além disto aplica-se no solo o Método da Rigidez Sucessiva (MRS), proposto em MAIER \& NOVATI (1987), estendendo-o ao caso tridimensional e à inclusão de subregiões, também tridimensionais. Estas sub-regiões simulam elementos de fundação tais como estacas, sapatas, tubulões, buracos ou túneis, podendo estes ultrapassar ou não as diferentes camadas de solo. A superestrutura, que pode ser até um edifício tridimensional, é simulada pelo MEF. Os pilares e vigas são representados por elementos reticulares e as lajes são consideradas diafragmas rígidos. O edifício pode ser apoiado em uma placa flexível (radier), que é modelada por elementos finitos laminares. Para avaliar a interação da superestrutura modelada pelo MEF com a subestrutura formulada pelo MEC, é feito o acoplamento a partir das matrizes provenientes de ambos subsistemas. O resultado é um sistema de equações que representa todo o conjunto.

A formulação de ALMEIDA (2003a) foi utilizada nos trabalhos de RIBEIRO, ALMEIDA \& PAIVA (2004) e ALMEIDA \& PAIVA (2004), para analisar problemas de interação do solo com a estrutura. Foram estudados problemas complexos em RIBEIRO, ALMEIDA \& PAIVA (2004), tais como um edifício composto por ele- 
mentos reticulares e membranas rígidas e um silo formado por elementos laminares, demonstrando a coerência da formulação.

Outros trabalhos envolvendo o tema da interação do solo com a estrutura e que

podem ser citados são SILVA (1994), MOURA (1999), LORENTZ (1985), MELLO (1984), VEIGA (2000), BARRETTO (1995), MENDONÇA (1997), IWAMOTO (2000), JORDÃO (2003), ALMEIDA (2003b), OSHIMA (2004) e PACCOLA (2004).

\subsection{Organização da dissertação}

O Capítulo 2 trata de maneira sucinta da formulação do Método dos Elementos de Contorno (MEC), voltando-a para para o campo de aplicação deste trabalho.

No Capítulo 3 é brevemente descrito o Método dos Elementos Finitos (MEF), abordando cada um dos diferentes tipos de elementos utilizados neste trabalho.

O Capítulo 4 contém o desenvolvimento do Método da Rigidez Sucessiva (MRS) aplicado ao maciço de solos, estendido para o caso tridimensional e com elementos de fundação.

O Capítulo 5 aborda a teoria desenvolvida para simular a interação do solo com a estrutura. São descritas a interação de um ou mais blocos tridimensionais com o solo, a interação de um edifício tridimensional com o solo e a interação do conjunto formado pelo solo, os blocos e um edifício tridimensional.

São apresentados, no Capítulo 6, exemplos de aplicação do programa, comparando os resultados obtidos com os de outros autores ou com o programa Ansys. Os exemplos mais complexos não tiveram seus resultados comparados devido à lacuna existente na literatura de análises mais completas.

O Capítulo 7 contém as conclusões do trabalho.

No Apêndice A é feita a dedução para se obter uma parcela específica da equação integral de contorno apresentada no capítulo 2. 


\section{Capítulo 2}

\section{O Método dos Elementos de Contorno}

\subsection{Introdução}

Este capítulo tem como objetivo apresentar sucintamente a formulação do Método dos Elementos de Contorno (MEC) para a análise estática de sólidos tridimensionais homogêneos em teoria linear. Este modelo é utilizado para simular as camadas de solo e os blocos no programa computacional deste trabalho, fazendo parte das extensões desenvolvidas pelo aluno.

A partir do equacionamento básico do problema elástico linear e de técnicas de resíduos ponderados chega-se à Identidade Somigliana, que é uma equação que permite o cálculo de deslocamentos em qualquer ponto do sólido. Deve-se então aplicar técnicas matemáticas para escrever a Identidade Somigliana para pontos do contorno do sólido. A equação resultante em conjunto com uma aproximação linear de deslocamento e força e as soluções fundamentais de Kelvin, LOVE (1944), permitem representar o problema por meio de um sistema de equações lineares, cuja solução são os valores incógnitos de contorno.

Resolvido o problema do valor de contorno, torna-se possível retornar à Identidade Somigliana e aplicar técnicas numéricas para calcular deslocamentos e tensões em pontos internos do sólido. Também são apresentadas técnicas de subelementação ao final do capítulo.

O MEC é descrito de forma semelhante à apresentada em BREBBIA \& DOMINGUEZ (1989). 


\subsection{Equacionamento básico do problema elástico linear}

Dado um sólido, seu comportamento se torna conhecido caso estejam determinados seus campos de tensões, deformações e deslocamentos. Assim, torna-se possível determinar-se o estado de tensão, deformação e deslocamento em qualquer ponto do sólido. Os dois primeiros são representados por tensores de segunda ordem, descritos a seguir, que podem ser relacionados por equações constitutivas. Estas relações são referentes às características do material do qual o sólido é formado.

\subsubsection{O estado de tensão}

O estado de tensão em um ponto $P$ qualquer de um sólido tridimensional, conforme mostrado na figura 2.1, é composto de nove componentes. Estas componentes podem ser agrupadas em um tensor, conforme a expressão 2.1.

$$
\sigma=\left[\begin{array}{lll}
\sigma_{11} & \sigma_{12} & \sigma_{13} \\
\sigma_{21} & \sigma_{22} & \sigma_{23} \\
\sigma_{31} & \sigma_{32} & \sigma_{33}
\end{array}\right]
$$
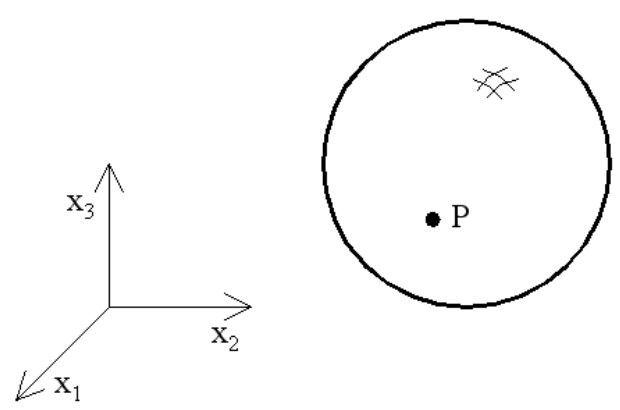

Figura 2.1: Sólido tridimensional

As componentes $\sigma_{i j}$ do tensor 2.1 obedecem condições de equilíbrio, que podem ser em momento ou em força. As equações de equilíbrio em momento podem ser escritas para um elemento infinitesimal bidimensional, conforme ilustrado na figura 2.2. Assim, obtém-se as relações:

$$
\begin{gathered}
\Sigma M=0 \\
\sigma_{i j} d x_{j} d x_{i}-\sigma_{j i} d x_{i} d x_{j}=0 \\
\sigma_{i j}=\sigma_{j i}
\end{gathered}
$$




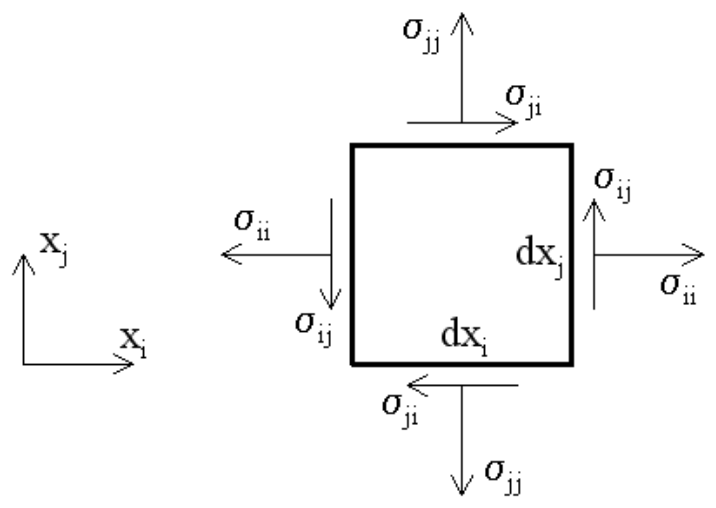

Figura 2.2: Equilíbrio em um elemento infinitesimal

Em um sólido tridimensional, a relação 2.4 é válida para quaisquer $i, j=1,2$, 3. Portanto, pode-se escrever as igualdades:

$$
\sigma_{12}=\sigma_{21}, \quad \sigma_{13}=\sigma_{31}, \quad \sigma_{23}=\sigma_{32}
$$

Isto reduz as componentes do estado de tensão de nove para seis. Mais equações podem ser obtidas a partir do equilíbrio de forças nas direções $x_{1}, x_{2}$ e $x_{3}$. A figura 2.3 ilustra o caso geral de uma parcela infinitesimal de um sólido tridimensional submetida a um estado de tensão e forças de domínio $b_{i}$ quaisquer. Considerando a soma de forças na direção do eixo $x_{1}$, conforme ilustrado na figura 2.3 , obtém-se a equação:

$$
\sum F x_{1}=0
$$

$\mathrm{Ou}$

$$
\sigma_{11}-\sigma_{11}-\frac{\partial \sigma_{11}}{\partial x_{1}} d x_{1}+\sigma_{12}-\sigma_{12}-\frac{\partial \sigma_{12}}{\partial x_{2}} d x_{1}+\sigma_{13}-\sigma_{13}-\frac{\partial \sigma_{13}}{\partial x_{3}} d x_{1}+b_{1} d x_{1}=0
$$
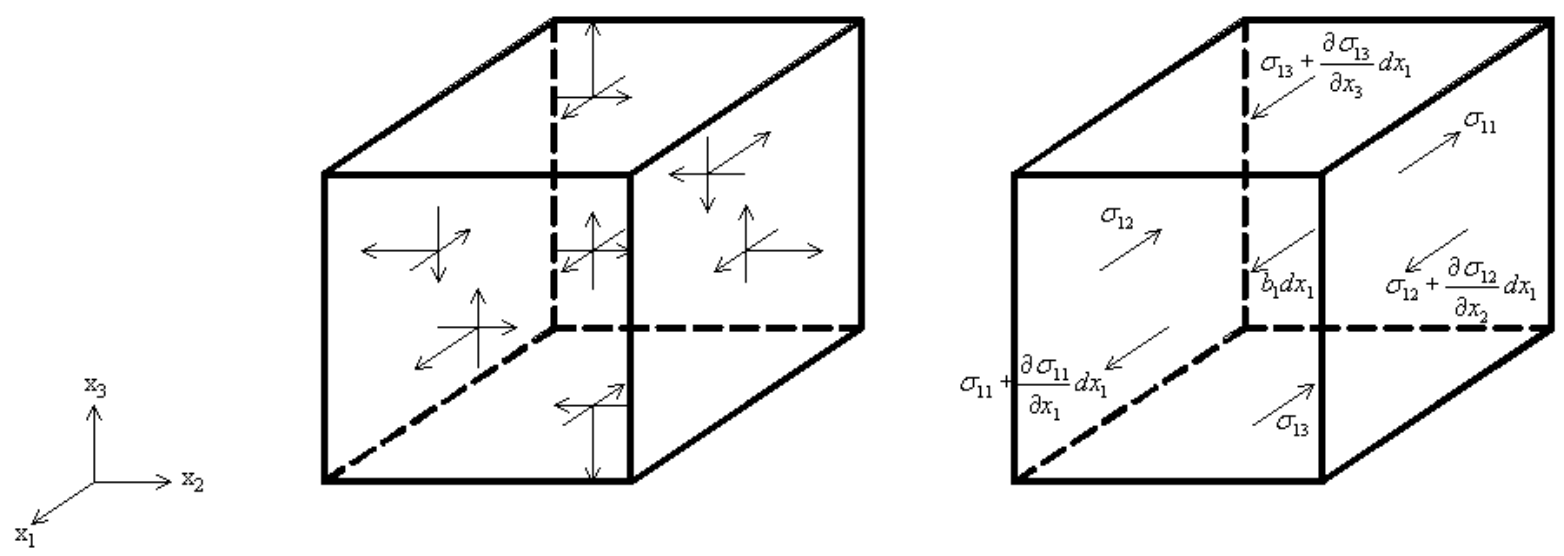

Figura 2.3: Equilíbrio em um elemento infinitesimal 
Desenvolvendo-se a equação 2.7 .

$$
\frac{\partial \sigma_{11}}{\partial x_{1}} d x_{1}+\frac{\partial \sigma_{12}}{\partial x_{2}} d x_{1}+\frac{\partial \sigma_{13}}{\partial x_{3}} d x_{1}+b_{1} d x_{1}=0
$$

E, por fim, obtém-se a equação de equilíbrio:

$$
\frac{\partial \sigma_{11}}{\partial x_{1}}+\frac{\partial \sigma_{12}}{\partial x_{2}}+\frac{\partial \sigma_{13}}{\partial x_{3}}+b_{1}=0
$$

O somatório de forças feito para a direção do eixo $x_{1}$ é válido também para as direções $x_{2}$ e $x_{3}$. Portanto:

$$
\begin{aligned}
& \frac{\partial \sigma_{21}}{\partial x_{1}}+\frac{\partial \sigma_{22}}{\partial x_{2}}+\frac{\partial \sigma_{23}}{\partial x_{3}}+b_{2}=0 \\
& \frac{\partial \sigma_{31}}{\partial x_{1}}+\frac{\partial \sigma_{32}}{\partial x_{2}}+\frac{\partial \sigma_{33}}{\partial x_{3}}+b_{3}=0
\end{aligned}
$$

Utilizando notação indicial, as equações 2.9, 2.10 e 2.11 se reduzem à seguinte igualdade:

$$
\sigma_{i j, j}+b_{i}=0
$$

Na sequência, é estudado o equilíbrio de um tetraedro definido pelos planos $x=0$, $y=0, z=0$ e um outro plano qualquer. Para que o tetraedro fique equilibrado em conjunto com o restante do sólido, aparecem componentes de força nas três direções cartesianas. Esta situação pode ser visualizada na figura 2.4 .

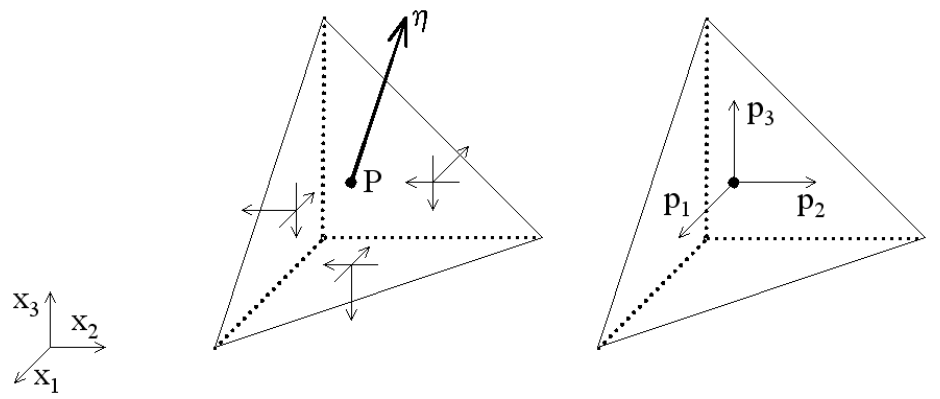

Figura 2.4: Equilíbrio de um tetraedro

Na figura 2.4, $p_{1}, p_{2}$ e $p_{3}$ são as componentes, nas direções dos eixos, da força por unidade de área resultante que equilibra o plano. O versor $\eta$ indica a orientação do plano e também pode ser decomposto nas direções cartesianas, obtendo $\eta_{1}, \eta_{2}$ e $\eta_{3}$. Estas componentes são denominadas co-senos diretores do plano. A partir da figura 2.4, escreve-se as expressões:

$$
p_{1}=\sigma_{11} \eta_{1}+\sigma_{12} \eta_{2}+\sigma_{13} \eta_{3}
$$




$$
\begin{aligned}
& p_{2}=\sigma_{21} \eta_{1}+\sigma_{22} \eta_{2}+\sigma_{23} \eta_{3} \\
& p_{3}=\sigma_{31} \eta_{1}+\sigma_{32} \eta_{2}+\sigma_{33} \eta_{3}
\end{aligned}
$$

As relações 2.13, 2.14 e 2.15 podem ser reduzidas a uma única expressão, utilizando notação indicial. Esta expressão é:

$$
p_{i}=\sigma_{i j} \eta_{j}
$$

Os co-senos diretores aparecem multiplicando as componentes do tensor de tensões porque estas atuam em parcelas dos planos $x=0, y=0$ e $z=0$, correspondentes às faces do tetraedro em estudo.

As equações 2.13, 2.14 e 2.15 são válidas para qualquer plano interno do sólido tridimensional em questão, no entanto geralmente são aplicadas ao seu contorno. Este contorno é uma superfície $\Gamma$, na qual deve-se prescrever valores de contorno que podem ser em força ou em deslocamento. No caso tridimensional, seis valores de contorno para cada ponto do contorno $\Gamma$, um deslocamento e uma força para cada direção cartesiana. Dado um ponto e uma direção, deve-se prescrever a força ou o deslocamento, resultando em um valor conhecido e seu conjugado incógnito.

\subsubsection{O estado de deformação}

O campo de deformações é função do campo de deslocamentos ao qual está sujeito o sólido. As relações entre estes campos estão mostradas a seguir:

$$
\begin{gathered}
\varepsilon_{11}=\frac{\partial u_{1}}{\partial x_{1}} \\
\varepsilon_{22}=\frac{\partial u_{2}}{\partial x_{2}} \\
\varepsilon_{33}=\frac{\partial u_{3}}{\partial x_{3}} \\
\varepsilon_{12}=\varepsilon_{21}=\frac{1}{2}\left(\frac{\partial u_{1}}{\partial x_{2}}+\frac{\partial u_{2}}{\partial x_{1}}\right) \\
\varepsilon_{13}=\varepsilon_{31}=\frac{1}{2}\left(\frac{\partial u_{1}}{\partial x_{3}}+\frac{\partial u_{3}}{\partial x_{1}}\right) \\
\varepsilon_{23}=\varepsilon_{32}=\frac{1}{2}\left(\frac{\partial u_{2}}{\partial x_{3}}+\frac{\partial u_{3}}{\partial x_{2}}\right)
\end{gathered}
$$

Caso se queira utilizar notação indicial, pode-se reduzir estas seis relações a uma só, que é:

$$
\varepsilon_{i j}=\frac{1}{2}\left(\frac{\partial u_{i}}{\partial x_{j}}+\frac{\partial u_{j}}{\partial x_{i}}\right)
$$


Assim como as tensões, as deformações podem ser agrupadas em um tensor, conforme mostrado a seguir:

$$
\varepsilon=\left[\begin{array}{lll}
\varepsilon_{11} & \varepsilon_{12} & \varepsilon_{13} \\
\varepsilon_{21} & \varepsilon_{22} & \varepsilon_{23} \\
\varepsilon_{31} & \varepsilon_{32} & \varepsilon_{33}
\end{array}\right]
$$

\subsubsection{Relações constitutivas}

O estado de tensões em um ponto qualquer pode ser relacionado ao estado de deformações no mesmo ponto por meio de relações constitutivas referentes ao material do sólido. Para problemas elásticos e lineares, podem ser definidas as constantes de Lamé $\lambda$ e $\mu$. Com estas constantes, pode-se encontrar o estado de tensões em um ponto a partir do estado de deformações com a expressão:

$$
\sigma_{i j}=\lambda \delta_{i j} \varepsilon_{k k}+2 \mu \varepsilon_{i j}
$$

Na expressão 2.25, o termo $\delta_{i j}$ corresponde à função Delta de Kronecker, que é igual a um quando $i$ é igual a $j$ e igual a zero quando $i$ é diferente de $j$. Caso se queira encontrar o estado de deformações a partir do estado de tensões, utiliza-se a expressão:

$$
\varepsilon_{i j}=\frac{-\lambda \delta_{i j}}{2 \mu(3 \lambda+2 \mu)} \sigma_{k k}+\frac{1}{2 \mu} \sigma_{i j}
$$

As constantes de Lamé estão relacionadas ao módulo de elasticidade longitudinal do material e ao coeficiente de Poisson pelas expressões:

$$
\begin{gathered}
\mu=\frac{E}{2(1+\nu)} \\
\lambda=\frac{\nu E}{(1+\nu)(1-2 \nu)}
\end{gathered}
$$

Na expressão 2.27, $E$ é o módulo de elasticidade longitudinal do material e $\nu$ é o coeficiente de Poisson. As expressões 2.25 e 2.26 podem então ser escritas em função de $E$ e $\nu$, se tornando:

$$
\begin{gathered}
\sigma_{i j}=\frac{E}{(1+\nu)}\left[\frac{\nu}{(1-2 \nu)} \delta_{i j} \varepsilon_{k k}+\varepsilon_{i j}\right] \\
\varepsilon_{i j}=\frac{-\nu}{E} \sigma_{k k} \delta_{i j}+\frac{1+\nu}{E} \sigma_{i j}
\end{gathered}
$$

$\mathrm{Na}$ análise feita nesta seção relativa ao problema elástico, foram definidas três componentes de deslocamento, seis componentes de tensão e seis componentes de deformação, totalizando quinze variáveis a serem determinadas. Para auxiliar na 
obtenção destas variáveis, foram escritas três equações de equilíbrio, seis equações de compatibilidade entre deslocamentos e deformações e a equação 2.25 ou 2.26 . Esta última está em notação indicial e representa seis equações constitutivas, somando com as demais quinze equações. Desta forma, com o número de equações igual ao número de incógnitas, torna-se possível determinar todas as componentes de deslocamento, deformação e tensão.

\subsection{Soluções fundamentais}

Nesta seção são apresentadas as soluções fundamentais para um meio infinito, tridimensional e elástico, resultantes de uma força concentrada e unitária aplicada em seu domínio, conforme feito por Kelvin e publicado em LOVE (1944).

Inicialmente, deve-se reescrever as equações de equilíbrio 2.12 em termos de deslocamentos. Para isto, substitui-se 2.29 em 2.12, obtendo:

$$
\frac{E}{(1+\nu)}\left[\frac{\nu}{(1-2 \nu)} \delta_{l j} \varepsilon_{m m, j}+\varepsilon_{l j, j}\right]+b_{l}=0
$$

Na sequência, substitui-se em 2.31 as relações entre deformação e deslocamento dadas por 2.23. Com isto, chega-se à equação:

$$
\frac{E}{(1+\nu)}\left[\frac{\nu}{(1-2 \nu)} \delta_{l j} u_{m, m j}+\frac{u_{l, j j}}{2}+\frac{u_{j, l j}}{2}\right]+b_{l}=0
$$

Dividindo 2.32 por $\mu$, constante de Lamé definida pela expressão 2.27, obtém-se:

$$
\frac{\nu}{(1-2 \nu)} u_{j, j l}+\frac{u_{l, j j}}{2}+\frac{u_{j, j l}}{2}+\frac{b_{l}}{\mu}=0
$$

Foi utilizada a propriedade $\delta_{l j} u_{m, m j}=u_{m, m l}$, e depois trocou-se o índice mudo $m$ por $j$. Desenvolvendo 2.33 , obtém-se a equação:

$$
\frac{1}{(1-2 \nu)} u_{j, j l}+u_{l, j j}+\frac{1}{\mu} b_{l}=0
$$

A expressão 2.34 representa as equações de equilíbrio de Navier, ou equações de equilíbrio em deslocamentos. A solução fundamental de Kelvin é obtida a partir da equação 2.34 quando uma força concentrada unitária é aplicada em um ponto $i$ na direção de um versor $e_{l}$, ou seja:

$$
b_{l}=\Delta^{i} e_{l}
$$

em que o termo $\Delta^{i}$ representa a função Delta de Dirac. Para tornar mais simples a dedução da solução fundamental, os deslocamentos podem ser representados pelo vetor de Galerkin. O deslocamento em uma determinada direção $j$ pode ser obtido 
a partir do vetor de Galerkin $G$ pela expressão:

$$
u_{j}=G_{j, m m}-\frac{1}{2(1-\nu)} G_{m, j m}
$$

onde $G$ é um vetor a ser determinado. Para prosseguir com o equacionamento, deve-se substituir as expressões 2.35 e 2.36 em 2.34. Assim:

$$
\frac{1}{(1-2 \nu)}\left[G_{j, m m j l}-\frac{1}{2(1-\nu)} G_{m, j m j l}\right]+\left[G_{l, m m j j}-\frac{1}{2(1-\nu)} G_{m, j m j j}\right]+\frac{1}{\mu} \Delta^{i} e_{l}=0
$$

Como o índice $m$ é mudo, ele pode ser trocado por qualquer outro. Na equação 2.37, troca-se alguns índices $m$ por $j$ e outros por $l$, de forma conveniente. Após a troca de índices, a equação 2.37 se torna:

$$
\frac{1}{(1-2 \nu)}\left[G_{j, j j j l}-\frac{1}{2(1-\nu)} G_{j, j j j l}\right]+\left[G_{l, j j l l}-\frac{1}{2(1-\nu)} G_{j, j j j l}\right]+\frac{1}{\mu} \Delta^{i} e_{l}=0
$$

Desenvolvendo a expressão 2.38, obtém-se a equação:

$$
\nabla^{2}\left(\nabla^{2} G_{l}\right)+\frac{1}{\mu} \Delta^{i} e_{l}=0
$$

Para problemas de estado plano de deformação, a expressão 2.39 pode ser escrita como:

$$
\nabla^{2}\left(F_{l}\right)+\frac{1}{\mu} \Delta^{i} e_{l}=0
$$

em que

$$
F_{l}=\nabla^{2} G_{l}
$$

A equação 2.40 é semelhante à que representa o problema potencial em BREBBIA \& DOMINGUEZ (1989). Por isso, ela admite a seguinte solução:

$$
F_{l}=\frac{1}{4 \pi r \mu} e_{l}
$$

em que $r$ é a distância entre o ponto de aplicação da carga unitária e o ponto para o qual $F_{l}$ está sendo calculado. O ponto de aplicação da carga é denominado "ponto fonte" e o ponto no qual é feito o cálculo é denominado "ponto campo". Substituindose a expressão 2.42 em 2.41, obtém-se:

$$
\nabla^{2} G_{l}=\frac{1}{4 \pi r \mu} e_{l}
$$


A solução desta equação é dada por:

$$
G_{l}=\frac{1}{8 \pi \mu} r e_{l}
$$

Tomando-se cada carga independentemente, pode-se escrever a igualdade:

$$
G_{l k}=G \delta_{l k}
$$

em que

$$
G=\frac{1}{8 \pi \mu} r
$$

e $\delta_{l k}$ é a função Delta de Kronecker. O índice $k$ se refere à componente do vetor de Galerkin e $l$ é a direção da carga unitária aplicada no ponto $i$. O deslocamento em um ponto qualquer do domínio, considerando cada direção como independente, pode ser escrito como:

$$
u_{k}^{*}=u_{l k}^{*} e_{l}
$$

em que $u_{k}^{*}$ é o deslocamento em qualquer ponto na direção $k$, quando é aplicada uma carga unitária no ponto $i$ e na direção $l$. De acordo com a definição dada por 2.36. pode-se escrever:

$$
u_{l k}^{*}=G_{l k, m m}-\frac{1}{2(1-\nu)} G_{l m, k m}
$$

O passo seguinte é substituir 2.45 e 2.46 em 2.48, obtendo:

$$
u_{l k}^{*}=\frac{1}{16 \pi \mu(1-\nu) r}\left[(3-4 \nu) \delta_{l k}+r_{, l} r_{, k}\right]
$$

A expressão 2.49 correponde à solução fundamental de deslocamento de Kelvin. A expressão 2.44 não é a única solução possível para a equação 2.40. Qualquer expressão de dimensão $r$ é viável, resultando em uma solução fundamental igual à da expressão 2.49, exceto por um movimeto de corpo rígido. Este pode ser desprezado por não alterar os campos de tensão e deformação do sólido.

O tensor de tensões em qualquer ponto interno pode ser encontrado substituindo a expressão 2.49 em 2.23 e o resultado em 2.29. A expressão resultante, representada em notação indicial, é:

$$
\sigma_{k j}^{*}=S_{l k j}^{*} e_{l}
$$

em que $S_{l k j}^{*}$ é um tensor. Substituindo-se a expressão 2.50 em 2.16, obtém-se o valor da tração em um ponto qualquer do contorno $\Gamma$, que é:

$$
p_{k}^{*}=p_{l k}^{*} e_{l}
$$


em que as componentes de tensão para o caso tridimensional são:

$$
p_{l k}^{*}=\frac{-1}{8 \pi(1-\nu) r^{2}}\left[\frac{\partial r}{\partial \eta}\left[(1-2 \nu) \delta_{l k}+3 r_{, l} r_{, k}\right]+(1-2 \nu)\left(\eta_{l} r_{, k}-\eta_{k} r_{, l}\right)\right]
$$

A expressão 2.52 corresponde à solução fundamental de força de Kelvin. Os termos $\eta_{l}$ e $\eta_{k}$ são os co-senos diretores relativos à normal $\eta$ e aos eixos cartesianos $x_{l}$ e $x_{k}$.

\subsection{Equações integrais de contorno}

Considera-se um sólido tridimensional, conforme ilustrado na figura 2.5. Nesta figura, o domínio do sólido é tratado por $\Omega$ e seu cotorno por $\Gamma$. Os pontos $s$ e $P$ são, respectivamente, o ponto fonte e o ponto campo. O ponto fonte é onde a carga unitária está aplicada e o ponto campo é onde se quer calcular valores. O contorno do sólido está dividido em dois trechos. No primeiro, denominado $\Gamma_{1}$, estão definidas as condições de contorno essenciais do problema. Isto significa que o deslocamento está prescrito em $\Gamma_{1}$, ou seja:

$$
u_{i}=\overline{u_{i}}
$$
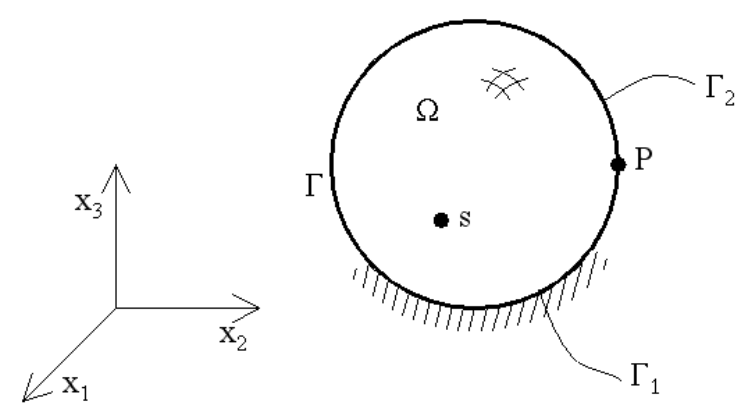

Figura 2.5: Condições de contorno arbitrárias

No segundo trecho, denominado $\Gamma_{2}$, estão definidas as condições de contorno naturais do problema. Isto significa que, em $\Gamma_{2}$, foi prescrito força, isto é:

$$
p_{i}=\overline{p_{i}}
$$

A equação integral governante do problema elástico será obtida pelo uso de técnicas de resíduos ponderados. O ponto de partida é a equação diferencial de equilíbrio em tensões deduzida anteriormente, que é:

$$
\sigma_{i j, j}+b_{i}=0
$$


Aplicando resíduos ponderados em 2.55, com uma função ponderadora em deslocamentos $u_{k}^{*}$, chega-se à expressão:

$$
\int_{\Omega}\left(\sigma_{k j, j}+b_{k}\right) u_{k}^{*} d \Omega=0
$$

A equação 2.56 também pode ser escrita como:

$$
\int_{\Omega}\left[\frac{\partial \sigma_{k 1}}{\partial x_{1}} u_{k}^{*}+\frac{\partial \sigma_{k 2}}{\partial x_{2}} u_{k}^{*}+\frac{\partial \sigma_{k 3}}{\partial x_{3}} u_{k}^{*}+b_{k} u_{k}^{*}\right] d \Omega=0
$$

Integrando 2.57 por partes, obtém-se:

$$
-\int_{\Omega}\left[\sigma_{k 1} \frac{\partial u_{k}^{*}}{\partial x_{1}}+\sigma_{k 2} \frac{\partial u_{k}^{*}}{\partial x_{2}}+\sigma_{k 3} \frac{\partial u_{k}^{*}}{\partial x_{3}}+b_{k} u_{k}^{*}\right] d \Omega+\int_{\Gamma} p_{k} u_{k}^{*} d \Gamma=0
$$

Na passagem de 2.57 para 2.58, foi utilizada a definição:

$$
\sigma_{k j} \eta_{j}=p_{k}
$$

Utilizando as equações de compatibilidade entre deslocamentos e deformações definidas pela expressão 2.23, pode-se reescrever 2.58 como:

$$
-\int_{\Omega} \sigma_{k j} \varepsilon_{k j}^{*} d \Omega+\int_{\Omega} b_{k} u_{k}^{*} d \Omega=-\int_{\Gamma} p_{k} u_{k}^{*} d \Gamma
$$

O teorema de Betti garante que:

$$
\int_{\Omega} \sigma_{k j} \varepsilon_{k j}^{*} d \Omega=\int_{\Omega} \sigma_{k j}^{*} \varepsilon_{k j} d \Omega
$$

Aplicando 2.61 em 2.60, obtém-se:

$$
-\int_{\Omega} \sigma_{k j}^{*} \varepsilon_{k j} d \Omega+\int_{\Omega} b_{k} u_{k}^{*} d \Omega=-\int_{\Gamma} p_{k} u_{k}^{*} d \Gamma
$$

Integrando 2.62 por partes, chega-se a uma equação similar a 2.56, que é:

$$
\int_{\Omega} \sigma_{k j, j}^{*} u_{k} d \Omega+\int_{\Omega} b_{k} u_{k}^{*} d \Omega=-\int_{\Gamma} p_{k} u_{k}^{*} d \Gamma+\int_{\Gamma} u_{k} p_{k}^{*} d \Gamma
$$

Imaginando o contorno $\Gamma$ dividido nas parcelas $\Gamma_{1}$ e $\Gamma_{2}$ nas quais são válidas as condições de contorno 2.53 e 2.54, reescreve-se 2.63 como:

$$
\int_{\Omega} \sigma_{k j, j}^{*} u_{k} d \Omega+\int_{\Omega} b_{k} u_{k}^{*} d \Omega=\int_{\Gamma_{1}} \bar{u}_{k} p_{k}^{*} d \Gamma+\int_{\Gamma_{2}} u_{k} p_{k}^{*} d \Gamma-\int_{\Gamma_{1}} p_{k} u_{k}^{*} d \Gamma-\int_{\Gamma_{2}} \bar{p}_{k} u_{k}^{*} d \Gamma
$$


Na sequência, deve-se integrar 2.64 por partes e aplicar o teorema de Betti de forma a retornar à expressão 2.56. Assim, integrando 2.64 por partes, chega-se à equação:

$$
\int_{\Omega} \sigma_{k j}^{*} \varepsilon_{k j} d \Omega+\int_{\Omega} b_{k} u_{k}^{*} d \Omega=\int_{\Gamma_{1}}\left(\bar{u}_{k}-u_{k}\right) p_{k}^{*} d \Gamma-\int_{\Gamma_{1}} p_{k} u_{k}^{*} d \Gamma-\int_{\Gamma_{2}} \bar{p}_{k} u_{k}^{*} d \Gamma
$$

Aplicando o teorema de Betti em 2.65, obtém-se:

$$
\int_{\Omega} \sigma_{k j} \varepsilon_{k j}^{*} d \Omega+\int_{\Omega} b_{k} u_{k}^{*} d \Omega=\int_{\Gamma_{1}}\left(\bar{u}_{k}-u_{k}\right) p_{k}^{*} d \Gamma-\int_{\Gamma_{1}} p_{k} u_{k}^{*} d \Gamma-\int_{\Gamma_{2}} \bar{p}_{k} u_{k}^{*} d \Gamma
$$

Integrando 2.66 por partes, obtém-se a igualdade:

$$
\int_{\Omega} \sigma_{k j, j} u_{k}^{*} d \Omega+\int_{\Omega} b_{k} u_{k}^{*} d \Omega=\int_{\Gamma_{1}}\left(\bar{u}_{k}-u_{k}\right) p_{k}^{*} d \Gamma+\int_{\Gamma_{2}}\left(p_{k}-\bar{p}_{k}\right) u_{k}^{*} d \Gamma
$$

A expressão 2.67 pode ser utilizada na obtenção das equações integrais de contorno. Para isto, deve-se aplicar as soluções fundamentais deduzidas anteriormente nesta expressão. Deste modo, a carga externa a ser considerada é uma força concentrada unitária aplicada em um ponto $i$ do domínio $\Omega$. A partir desta hipótese, a equação diferencial de equilíbrio em tensões 2.55 pode ser escrita como:

$$
\sigma_{k j, j}^{*}+\Delta^{i} e_{l}=0
$$

$\mathrm{Ou}$

$$
\sigma_{k j, j}^{*}=-\Delta^{i} e_{l}
$$

em que $\Delta^{i}$ é a função Delta de Dirac concentrada no ponto $i$ de aplicação da carga. A igualdade 2.69 pode ser utilizada na primeira integral à esquerda de 2.67, que se torna:

$$
\int_{\Omega} \sigma_{k j, j}^{*} u_{k} d \Omega=\int_{\Omega}\left(-\Delta^{i} e_{l}\right) u_{l} d \Omega=-u_{l}^{i} e_{l}
$$

Nas igualdades 2.70, $u_{l}^{i}$ é o deslocamento no ponto $i$ onde a carga unitária foi aplicada e na direção $l$. Substituindo 2.70 em 2.67, chega-se à equação:

$$
u_{l}^{i} e_{l}+\int_{\Gamma_{1}} \bar{u}_{k} p_{l k}^{*} d \Gamma e_{l}+\int_{\Gamma_{2}} u_{k} p_{l k}^{*} d \Gamma e_{l}=\int_{\Gamma_{1}} p_{k} u_{l k}^{*} d \Gamma e_{l}+\int_{\Gamma_{2}} \bar{p}_{k} u_{l k}^{*} d \Gamma e_{l}+\int_{\Omega} b_{k} u_{l k}^{*} d \Omega e_{l}
$$

O versor $e_{l}$ aparece em todos os termos, portanto pode ser excluído. Assim:

$$
u_{l}^{i}+\int_{\Gamma_{1}} \bar{u}_{k} p_{l k}^{*} d \Gamma+\int_{\Gamma_{2}} u_{k} p_{l k}^{*} d \Gamma=\int_{\Gamma_{1}} p_{k} u_{l k}^{*} d \Gamma+\int_{\Gamma_{2}} \bar{p}_{k} u_{l k}^{*} d \Gamma+\int_{\Omega} b_{k} u_{l k}^{*} d \Omega
$$


A equação 2.72 pode ser escrita de forma mais compacta caso as condições de contorno sejam, por enquanto, ignoradas. Desta forma:

$$
u_{l}^{i}+\int_{\Gamma} u_{k} p_{l k}^{*} d \Gamma=\int_{\Gamma} p_{k} u_{l k}^{*} d \Gamma+\int_{\Omega} b_{k} u_{l k}^{*} d \Omega
$$

A equação 2.73 é chamada de Indentidade Somigliana. Por meio dela, é possível determinar componentes de deslocamento em qualquer ponto do domínio $\Omega \mathrm{em}$ função dos valores de contorno $u_{k}$ e $p_{k}$. É necessário que também sejam conhecidas as soluções fundamentais em deslocamento $u_{l k}^{*}$ e força $p_{l k}^{*}$, que foram deduzidas anteriormente, além das cargas externas de domínio $b_{k}$.

\subsubsection{Equação integral para pontos do contorno}

Para determinar as componentes de deslocamento em um ponto $i$ do domínio $\Omega$ a partir da Identidade Somigliana 2.73, devem ser conhecidos os valores de contorno dados por $u_{k}$ e $p_{k}$. Portanto, para calcular valores em pontos internos do sólido devese primeiramente resolver o problema do valor de contorno. Como a equação 2.73 é válida somente no domínio $\Omega$ e não no contorno $\Gamma$, deve-se obter uma equação integral de contorno levando-se 2.73 para o contorno. Neste procedimento, cada termo deve ser analisado separadamente para tratar de forma especial as integrais que se tornem singulares. Para maior simplicidade, inicialmente é considerado que o contorno $\Gamma$ é suave no ponto $i$ analisado. Com isto, torna-se possível englobar o ponto $i$ ao interior de $\Omega$ por meio de uma semi-esfera, cujo raio $\varepsilon$ será posteriormente levado a zero no limite. Isto está ilustrado na figura 2.6.

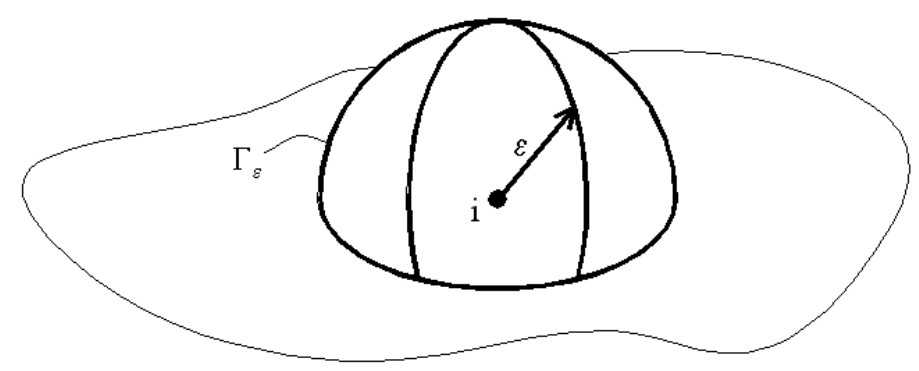

Figura 2.6: Ponto $i$ envolvido por uma semi-esfera de raio $\varepsilon$

Iniciando a análise, toma-se a primeira integral à direita da equação 2.73. Conforme ilustrado na figura 2.6, a semi-esfera contribui com uma parcela $\Gamma_{\varepsilon}$ do contorno Г. Assim, esta integral pode ser dividida em duas partes:

$$
\int_{\Gamma} u_{l k}^{*} p_{k} d \Gamma=\lim _{\varepsilon \rightarrow 0}\left\{\int_{\Gamma-\Gamma_{\varepsilon}} u_{l k}^{*} p_{k} d \Gamma\right\}+\lim _{\varepsilon \rightarrow 0}\left\{\int_{\Gamma_{\varepsilon}} u_{l k}^{*} p_{k} d \Gamma\right\}
$$


$\mathrm{O}$ primeiro limite à direita de 2.74 torna $\Gamma_{\varepsilon}$ infinitamente menor que $\Gamma$, o que leva à conclusão de que este limite é igual à própria integral inicial. O segundo limite à direita pode ser escrito como:

$$
\lim _{\varepsilon \rightarrow 0}\left\{\int_{\Gamma_{\varepsilon}} u_{l k}^{*} p_{k} d \Gamma\right\}=p_{k}^{i} \lim _{\varepsilon \rightarrow 0}\left\{\int_{\Gamma_{\varepsilon}} u_{l k}^{*} d \Gamma\right\}
$$

em que $p_{k}^{i}$ é uma força no ponto $i$ na direção $k$. A solução fundamental $u_{l k}^{*}$ tem dimensão $1 / r$ ou $1 / \varepsilon$, conforme demonstrado na expressão 2.49. Como a integral de superfície na expressão 2.74 produz um termo $\varepsilon^{2}$ multiplicando a expressão, restará do desenvolvimento desta equação um $\varepsilon$ multiplicando toda a expressão. Isto é suficiente para concluir que este termo tende a zero no limite. Portanto, a primeira integral à direita de 2.73 não sofre influência da singularidade no ponto $i$.

A próxima integral a ser analisada é a da esquerda da expressão 2.73 .

$$
\int_{\Gamma} p_{l k}^{*} u_{k} d \Gamma=\lim _{\varepsilon \rightarrow 0}\left\{\int_{\Gamma-\Gamma_{\varepsilon}} p_{l k}^{*} u_{k} d \Gamma\right\}+\lim _{\varepsilon \rightarrow 0}\left\{\int_{\Gamma_{\varepsilon}} p_{l k}^{*} u_{k} d \Gamma\right\}
$$

O primeiro limite à direita de 2.76 se comporta da mesma maneira que o primeiro limite à direita de 2.74, portanto é igual à integral à esquerda de 2.76. O segundo limite à direita de 2.76 pode ser escrito como:

$$
\lim _{\varepsilon \rightarrow 0}\left\{\int_{\Gamma_{\varepsilon}} p_{l k}^{*} u_{k} d \Gamma\right\}=u_{k}^{i} \lim _{\varepsilon \rightarrow 0}\left\{\int_{\Gamma_{\varepsilon}} p_{l k}^{*} d \Gamma\right\}
$$

A solução fundamental $p_{l k}^{*}$, dada pela expressão 2.52, tem dimensão $1 / r^{2}$, enquanto a integral de superfície de 2.77 produz um termo $\varepsilon^{2}$ multiplicando toda a expressão. Isto significa que o limite 2.77 não é igual a zero, e sim um termo livre. Para encontrar este termo é necessário desenvolver analiticamente a integral 2.77. No apêndice A está exposta esta dedução. Após os procedimentos matemáticos, chega-se à igualdade:

$$
\lim _{\varepsilon \rightarrow 0}\left\{\int_{\Gamma_{\varepsilon}} p_{l k}^{*} d \Gamma\right\}=-\frac{1}{2} \delta_{l k}
$$

Portanto, a expressão 2.76 se torna:

$$
\int_{\Gamma} p_{l k}^{*} u_{k} d \Gamma=\int_{\Gamma} p_{l k}^{*} u_{k} d \Gamma-\frac{1}{2} \delta_{l k} u_{k}^{i}=\int_{\Gamma} p_{l k}^{*} u_{k} d \Gamma-\frac{1}{2} u_{l}^{i}
$$

A integral de 2.79 é definida como Valor Principal de Cauchy.

Após estas análises, pode-se retornar à expressão 2.73 e reescrevê-la da seguinte 
forma:

$$
c_{l k}^{i} u_{k}^{i}+\int_{\Gamma} p_{l k}^{*} u_{k} d \Gamma=\int_{\Gamma} u_{l k}^{*} p_{k} d \Gamma+\int_{\Omega} u_{l k}^{*} b_{k} d \Omega
$$

Cada integral da expressão 2.80 é definida como Valor Principal de Cauchy e, para o caso de $i$ se encontrar em um trecho suave de $\Gamma$, o termo $c_{l k}^{i}$ é igual à função Delta de Kronecker dividida por dois.

Quando o ponto $i$ se encontra em um trecho de $\Gamma$ que não é suave, as integrais da equação 2.79 levam a diferentes valores de $c_{l k}^{i}$, tornando difícil encontrar uma expressão geral para este termo. No entanto, calcular este valor analiticamente não é necessário, pois ele pode ser obtido aplicando movimentos de corpo rígido ao sólido. Isto é explicado mais adiante, na dedução das matrizes $H$ e $G$.

A equação 2.80 permite resolver o problema de valor de contorno para o problema elástico tridimensional. Em cada ponto definido em $\Gamma$ e para cada direção, devese prescrever um valor de contorno em força ou deslocamento. Caso em todos os pontos de $\Gamma$ os deslocamentos sejam prescritos, 2.80 produz uma equação integral de primeiro tipo. Caso o que esteja prescrito em todos os pontos seja força, então a equação integral se torna de segundo tipo. A combinação de ambas as condições de contorno, que é o mais usual, resulta em uma equação integral mista.

\subsubsection{Formulação de elementos de contorno}

A equação 2.80 pode ser resolvida numericamente dividindo-se o contorno $\Gamma$ em elementos. Neste trabalho são utilizados elementos planos triangulares, conforme mostrado na figura 2.7. Nesta figura, a superfície do elemento encontra-se representada por $\Gamma_{e}$. Em cada nó do elemento, estão definidos seis graus de liberdade. Como o sistema cartesiano é de três eixos, $x_{1}, x_{2}$ e $x_{3}$, cada nó tem três componentes de deslocamento $u$ e três componentes de força $p$, indicados na figura. Nestes valores, o índice subscrito indica a direção cartesiana e o índice sobrescrito indica a numeração local do nó.

Ao longo de cada elemento deve ser definida uma função para representar os deslocamentos e as forças a partir de seus valores nodais. Ao escrever 2.80 para cada ponto nodal, é obtido um sistema de equações lineares, ao qual devem ser aplicadas as condições de contorno do problema. Então o sistema pode ser resolvido, obtendo-se todos os deslocamentos e forças incógnitos no contorno. Estes valores, em conjunto com as condições de contorno prescritas, constituem a solução do problema de valor de contorno.

Inicialmente, representa-se os deslocamentos $u$ e forças $p$ por funções conhecidas. Estas funções, contínuas ao longo de cada elemento $j$, podem ser escritas em forma 


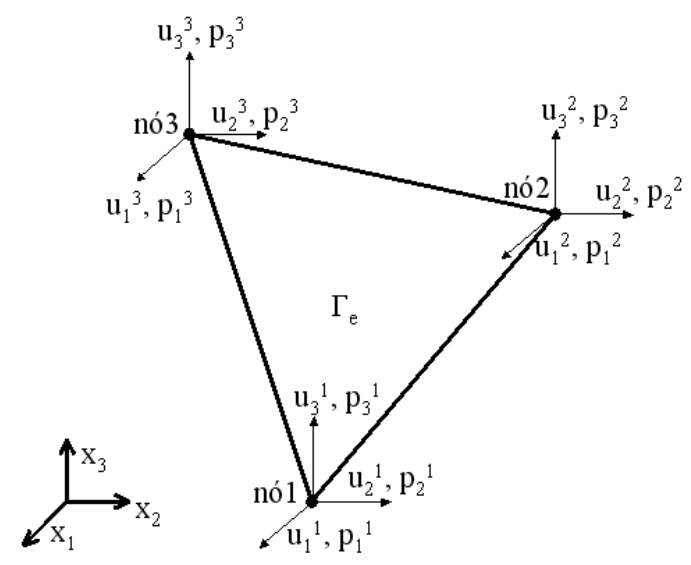

Figura 2.7: Elemento de contorno triangular

matricial como:

$$
\begin{aligned}
& \mathbf{u}=\boldsymbol{\Phi} \mathbf{u}^{j} \\
& \mathbf{p}=\boldsymbol{\Phi} \mathbf{p}^{j}
\end{aligned}
$$

Os vetores $\mathbf{u}^{\mathbf{j}}$ e $\mathbf{p}^{\mathbf{j}}$ são, respectivamente, os deslocamentos e forças estabelecidos nos nós do elemento $j$. Para o caso tridimensional estes vetores têm $3 Q$ linhas, sendo $Q$ o número de nós por elemento. Os vetores $u$ e $p$ contêm, respectivamente, os deslocamentos e forças nas três direções em um ponto qualquer da superfície $\Gamma_{e}$ do elemento. Ou seja:

$$
\begin{gathered}
\mathbf{u}=\left\{\begin{array}{l}
u_{1} \\
u_{2} \\
u_{3}
\end{array}\right\} \\
\mathbf{p}=\left\{\begin{array}{l}
p_{1} \\
p_{2} \\
p_{3}
\end{array}\right\}
\end{gathered}
$$

A matriz de interpolação $\boldsymbol{\Phi}$ tem dimensão $3 \times 3 Q$ e possui $Q$ funções $\phi$ diferentes. Para o elemento de contorno de três nós da figura 2.7, a matriz $\Phi$ é:

$$
\boldsymbol{\Phi}=\left[\begin{array}{ccccccccc}
\phi_{1} & 0 & 0 & \phi_{2} & 0 & 0 & \phi_{3} & 0 & 0 \\
0 & \phi_{1} & 0 & 0 & \phi_{2} & 0 & 0 & \phi_{3} & 0 \\
0 & 0 & \phi_{1} & 0 & 0 & \phi_{2} & 0 & 0 & \phi_{3}
\end{array}\right]
$$

e o vetor $\mathbf{u}^{\mathbf{j}}$ é:

$$
\left\{\mathbf{u}^{j}\right\}^{T}=\left\{\begin{array}{lllllllll}
u_{1}^{1} & u_{2}^{1} & u_{3}^{1} & u_{1}^{2} & u_{2}^{2} & u_{3}^{2} & u_{1}^{3} & u_{2}^{3} & u_{3}^{3}
\end{array}\right\}
$$


Portanto, o deslocamento em um ponto qualquer de $\Gamma_{e}$ pode ser escrito como:

$$
\mathbf{u}=\left\{\begin{array}{l}
u_{1} \\
u_{2} \\
u_{3}
\end{array}\right\}=\left\{\begin{array}{c}
u_{1}^{1} \phi_{1}+u_{1}^{2} \phi_{2}+u_{1}^{3} \phi_{3} \\
u_{2}^{1} \phi_{1}+u_{2}^{2} \phi_{2}+u_{2}^{3} \phi_{3} \\
u_{3}^{1} \phi_{1}+u_{3}^{2} \phi_{2}+u_{3}^{3} \phi_{3}
\end{array}\right\}
$$

Pode-se observar, pela igualdade 2.87, que o deslocamento em uma direção de um ponto qualquer do elemento $j$ é função dos deslocamentos nesta direção dos três nós do elemento. Observa-se também que as mesmas funções são utilizadas nas três direções.

A igualdade 2.87 também é válida para as forças, ou seja:

$$
\mathbf{p}=\left\{\begin{array}{c}
p_{1} \\
p_{2} \\
p_{3}
\end{array}\right\}=\left\{\begin{array}{c}
p_{1}^{1} \phi_{1}+p_{1}^{2} \phi_{2}+p_{1}^{3} \phi_{3} \\
p_{2}^{1} \phi_{1}+p_{2}^{2} \phi_{2}+p_{2}^{3} \phi_{3} \\
p_{3}^{1} \phi_{1}+p_{3}^{2} \phi_{2}+p_{3}^{3} \phi_{3}
\end{array}\right\}
$$

São adotadas funções $\phi$ lineares, conforme mostrado na figura 2.8 .

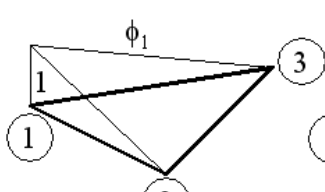

(2)

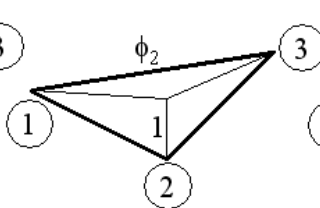

(2)

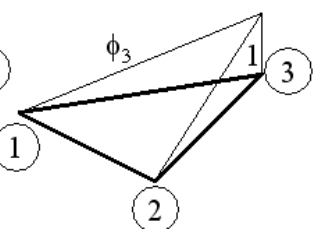

(2)

Figura 2.8: Funções interpoladoras adotadas

Para tornar a resolução das integrais mais simples, as funções $\phi_{1}, \phi_{2}$ e $\phi_{3}$ são definidas segundo um sistema de coordenadas local. Este sistema, juntamente com o global, está ilustrado na figura 2.9.

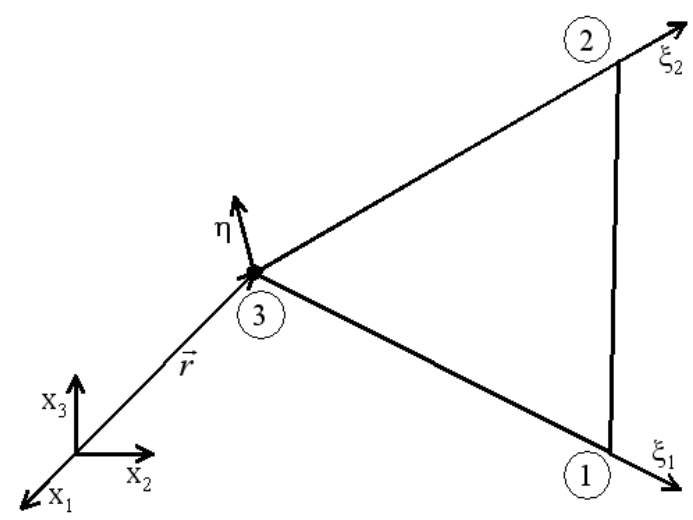

Figura 2.9: Sistemas de coordenadas global e local

Por serem lineares, as fuções $\phi_{1}, \phi_{2}$ e $\phi_{3}$ devem ser do tipo:

$$
\phi_{i}\left(\xi_{1}, \xi_{2}\right)=\alpha \xi_{1}+\beta \xi_{2}+\chi
$$


em que $\alpha, \beta$ e $\chi$ são constantes e $\xi_{1}$ e $\xi_{2}$ são coordenadas adimensionais. Uma opção para determinar estas constantes é obter equações a partir da figura 2.8. Assim:

$$
\begin{gathered}
\xi_{1}=0 \rightarrow \phi_{1}=1, \quad \phi_{2}=0, \quad \phi_{3}=0 \\
\xi_{2}=0 \rightarrow \phi_{1}=0, \quad \phi_{2}=1, \quad \phi_{3}=0 \\
\xi_{1}=0 \quad e \quad \xi_{2}=0 \rightarrow \phi_{1}=0, \quad \phi_{2}=0, \quad \phi_{3}=1
\end{gathered}
$$

A partir das equações 2.90, 2.91 e 2.92, pode-se concluir que:

$$
\begin{gathered}
\phi_{1}=\xi_{1} \\
\phi_{2}=\xi_{2} \\
\phi_{3}=-\xi_{1}-\xi_{2}+1
\end{gathered}
$$

Além dos deslocamentos $\mathbf{u}$ e das forças $\mathbf{p}$, também devem ser representadas de forma matricial as cargas volumétricas e as soluções fundamentais. As forças externas de domínio podem ser organizadas em um vetor que contenha suas componentes, ou seja:

$$
\mathbf{b}=\left\{\begin{array}{l}
b_{1} \\
b_{2} \\
b_{3}
\end{array}\right\}
$$

As soluções fundamentais podem ser representadas em forma matricial como segue:

$$
\begin{aligned}
& \mathbf{u}^{*}=\left[\begin{array}{lll}
u_{11}^{*} & u_{12}^{*} & u_{13}^{*} \\
u_{21}^{*} & u_{22}^{*} & u_{23}^{*} \\
u_{31}^{*} & u_{32}^{*} & u_{33}^{*}
\end{array}\right] \\
& \mathbf{p}^{*}=\left[\begin{array}{lll}
p_{11}^{*} & p_{12}^{*} & p_{13}^{*} \\
p_{21}^{*} & p_{22}^{*} & p_{23}^{*} \\
p_{31}^{*} & p_{32}^{*} & p_{33}^{*}
\end{array}\right]
\end{aligned}
$$

Os coeficientes subscritos dos termos $u_{l k}^{*}$ e $p_{l k}^{*}$ das matrizes indicam a direção $l$ da carga e a direção $k$ do deslocamento ou força.

Aplicando a notação matricial apresentada, a equação 2.80 se torna:

$$
\mathrm{c}^{\mathrm{i}} \mathbf{u}^{\mathrm{i}}+\int_{\Gamma} \mathrm{p}^{*} \mathrm{ud} \Gamma=\int_{\Gamma} \mathbf{u}^{*} \operatorname{pd} \Gamma+\int_{\Omega} \mathbf{u}^{*} \mathrm{bd} \Omega
$$

Considerando agora que as funções de forma $\boldsymbol{\Phi}$ sejam substituídas na equação 2.99, chega-se a uma equação onde são discriminadas as influências de cada elemento, 
isto é:

$$
\mathbf{c}^{\mathrm{i}} \mathbf{u}^{\mathrm{i}}+\sum_{\mathbf{j}=\mathbf{1}}^{\mathrm{ne}}\left[\left(\int_{\Gamma_{\mathbf{j}}} \mathrm{p}^{*} \boldsymbol{\Phi} \mathrm{d} \Gamma\right) \mathbf{u}^{\mathrm{j}}\right]=\sum_{\mathbf{j}=\mathbf{1}}^{\mathrm{ne}}\left[\left(\int_{\Gamma_{\mathbf{j}}} \mathbf{u}^{*} \Phi \mathrm{d} \Gamma\right) \mathbf{p}^{\mathrm{j}}\right]
$$

em que ne é o número de elementos definido no contorno. Foi considerado na equação 2.100 que as forças externas de domínio, $b$, são nulas. É somada a influência da superfície $\Gamma_{j}$ de cada elemento de um até o número de elementos, ne. Os vetores $\mathbf{u}^{\mathbf{j}}$ e $\mathbf{p}^{\mathbf{j}}$ contêm, respectivamente, os deslocamentos e forças nos nós do elemento $j$.

\subsubsection{Transformação de coordenadas}

O sistema de coordenadas local pode ser relacionado ao global utilizando um Jacobiano, como mostrado abaixo:

$$
d \Gamma=|\mathbf{J}| d \xi_{1} d \xi_{2}
$$

Substituindo a expressão 2.101 na equação 2.100, obtém-se:

$$
\mathbf{c}^{\mathbf{i}} \mathbf{u}^{\mathbf{i}}+\sum_{\mathbf{j}=\mathbf{1}}^{\mathbf{n e}}\left[\left\{\int_{\Gamma_{\mathbf{j}}} \mathbf{p}^{*} \boldsymbol{\Phi}|\mathbf{J}| d \xi_{1} d \xi_{2}\right\} \mathbf{u}^{\mathbf{j}}\right]=\sum_{\mathbf{j}=\mathbf{1}}^{\mathbf{n}}\left[\left\{\int_{\Gamma_{\mathbf{j}}} \mathbf{u}^{*} \boldsymbol{\Phi}|\mathbf{J}| d \xi_{1} d \xi_{2}\right\} \mathbf{p}^{\mathbf{j}}\right]
$$

As integrais da equação 2.102 são calculadas numericamente. O resultado é a expressão:

$$
\mathbf{c}^{\mathbf{i}} \mathbf{u}^{\mathbf{i}}+\sum_{\mathbf{j}=1}^{\mathrm{ne}}\left[\left\{\sum_{\mathbf{k}=1}^{\mathrm{l}} \mathbf{w}_{\mathbf{k}}\left(\mathbf{p}^{*} \boldsymbol{\Phi}\right)_{\mathbf{k}}|\mathbf{J}|\right\} \mathbf{u}^{\mathbf{j}}\right]=\sum_{\mathbf{j}=1}^{\mathrm{ne}}\left[\left\{\sum_{\mathbf{k}=1}^{\mathrm{l}} \mathbf{w}_{\mathbf{k}}\left(\mathbf{u}^{*} \boldsymbol{\Phi}\right)_{\mathbf{k}}|\mathbf{J}|\right\} \mathbf{p}^{\mathbf{j}}\right]
$$

O termo $l$ se refere ao número de pontos de integração definidos sobre a superfície dos elementos de contorno. O peso de cada ponto é definido pela função $w_{k}$.

\subsubsection{Sistema de equações}

A equação 2.103 é válida para um nó $i$ qualquer pertencente ao domínio $\Omega$ do sólido. Para simplificar a implementação desta equação, é mais interessante que os somatórios sejam em nós ao invés de elementos. Para efetuar esta transformação, são definidas as relações:

$$
\begin{aligned}
& \hat{\mathbf{H}}_{\mathrm{ij}}=\sum_{\mathrm{t}} \int_{\Gamma_{\mathrm{t}}} \mathrm{p}^{*} \Phi_{\mathrm{q}} \mathrm{d} \Gamma \\
& \mathrm{G}_{\mathrm{ij}}=\sum_{\mathrm{t}} \int_{\Gamma_{\mathrm{t}}} \mathbf{u}^{*} \Phi_{\mathrm{q}} \mathrm{d} \Gamma
\end{aligned}
$$

Nestas relações, o índice $i$ é o número do nó onde se encontra o ponto campo e o índice $j$ é o número do nó onde se encontra o ponto fonte. O índice $q$ indica a 
numeração local do nó $j$ no elemento $t$. A soma referenciada por $t$ vai de 1 até o número de elementos dos quais o nó $j$ é vértice.

Assim, com as relações 2.104 e 2.105 , pode-se reescrever a equação 2.103 como:

$$
c^{i} u^{i}+\sum_{j=1}^{3 n} \hat{H}_{i j} u^{j}=\sum_{j=1}^{3 n} G_{i j} p^{j}
$$

em que $n$ é o número de nós no contorno. Para simplificar a equação 2.106, impõemse as condições:

$$
\begin{gathered}
\mathbf{H}_{\mathbf{i} \mathbf{j}}=\hat{\mathbf{H}}_{\mathbf{i j}} \text { para } i \neq j \\
\mathbf{H}_{\mathbf{i j}}=\hat{\mathbf{H}}_{\mathbf{i j}}+\mathbf{c}^{\mathbf{i}} \text { para } i=j
\end{gathered}
$$

Substituindo $\mathbf{H}_{\mathbf{i j}}$, dado nas relações 2.107 e 2.108, na equação 2.106, obtém-se:

$$
\sum_{j=1}^{3 n} H_{i j} u^{j}=\sum_{j=1}^{3 n} G_{i j} p^{j}
$$

A equação 2.109 pode ser escrita para todos os nós do contorno, resultando um sistema de $3 n$ equações cuja forma matricial é:

$$
[\mathbf{H}]\{\mathbf{u}\}=[\mathbf{G}]\{\mathbf{p}\}
$$

Conforme mostrado nas relações 2.107 e 2.108, os termos $\mathbf{c}^{\mathbf{i}}$ são computados na matriz H. Eles compõem uma série de submatrizes de dimensão $3 \times 3$, somadas na região da diagonal principal de $\mathbf{H}$. Para encontrar os valores das submatrizes $\mathbf{c}^{\mathbf{i}}$ em casos gerais nos quais o contorno $\Gamma$ não é suave, pode-se aplicar propriedades da matriz $\mathbf{H}$ decorrentes de movimentos de corpo rígido. Isto será visto mais adiante, na seção 2.4.5.

Para que se possa resolver o problema do valor de contorno a partir das equações representadas por 2.110, é necessário adaptar este sistema de equações às condições de contorno do problema. Isto pode ser feito prescrevendo, para cada direção de cada ponto do contorno, um valor de deslocamento ou um valor de força. Desta forma, ambos os vetores $\mathbf{u}$ e $\mathbf{p}$ se tornam parcialmente conhecidos e parcialmente incógnitos. Além disto, caso o valor de deslocamento em uma determinada linha de $\mathbf{u}$ seja prescrito, o valor nesta mesma linha de $\mathbf{p}$ obrigatoriamente deve estar incógnito e vice-versa. Desta forma, o número de incógnitas se torna igual ao número de equações e o sistema tem solução única.

Para obter os valores de contorno incógnitos na equação 2.110 , é necessário que todos eles estejam no mesmo lado da equação. Da mesma forma, todos os valores prescritos devem estar do outro lado. Isto pode ser feito a partir da equação 2.110. passando para o lado esquerdo cada valor incógnito de $\mathbf{p}$ e o substituindo no lado 
direito pelo correspondente conhecido de $\mathbf{u}$. As matrizes $\mathbf{H}$ e $\mathbf{G}$ também devem ser modificadas, trocando entre elas a coluna correspondente à linha de $\mathbf{p}$ e $\mathbf{u}$ para a qual foi feita a troca. Repetindo este procedimento para todas as forças incógnitas, obtém-se a equação:

$$
\mathrm{Ax}=\mathrm{By}
$$

$\mathrm{Na}$ equação 2.111, todos os termos do vetor $\mathbf{x}$ são incógnitos e todos os termos do vetor $\mathbf{y}$ são conhecidos. Além disto, todos os termos das matrizes $\mathbf{A}$ e $\mathbf{B}$ também são conhecidos, pois elas são resultado da troca de colunas entre as matrizes $\mathbf{H}$ e $\mathbf{G}$. Por isto, pode-se fazer o produto entre a matriz $\mathbf{B}$ e o vetor $\mathbf{y}$ à direita da igualdade. O resultado é um sistema de $3 n$ equações com $3 n$ incógnitas, ou seja:

$$
\mathrm{Ax}=\mathbf{f}
$$

Pela resolução do sistema 2.112 podem ser obtidos todos os valores de contorno incógnitos, resolvendo o problema elástico tridimensional do valor de contorno.

\subsubsection{Movimentos de corpo rígido}

O conceito de movimento de corpo rígido envolve deslocamentos na ausência de forças. Isto implica em prescrever todas as forças no contorno $\Gamma$ do sólido iguais a zero. Ao fazer isto na equação 2.110, o produto Gp fica igual a zero. Assim:

$$
\mathbf{H u}=\mathbf{0}
$$

A interpretação física do problema de valor de contorno representado pela equação 2.113 é de um sólido sem restrição alguma, portanto solto no espaço. Extendendo esta interpretação, uma configuração possível é deslocar todos os nós do contorno do sólido de uma distância unitária no sentido positivo de um dos eixos coordenados. Escolhendo o eixo $x_{1}$, por exemplo, o vetor $\mathbf{u}_{\mathbf{1}}$ para um movimento de corpo rígido unitário positivo em sua direção é:

$$
\left\{\mathbf{u}_{1}\right\}^{T}=\left\{\begin{array}{llllllllll}
1 & 0 & 0 & 1 & 0 & 0 & \cdots & 1 & 0 & 0
\end{array}\right\}
$$

O produto de uma linha $i$ qualquer da matriz $\mathbf{H}$ pelo vetor $\mathbf{u}_{\mathbf{1}}$ deve ser igual a zero, conforme imposto na equação 2.113. Assim, obtém-se uma importante propriedade da matriz $\mathbf{H}$, que é:

$$
H_{1}^{i}+H_{4}^{i}+H_{7}^{i}+\cdots+H_{(3 n-2)}^{i}=0
$$


O mesmo pode ser feito para as direções de $x_{2}$ e $x_{3}$, obtendo outras duas equações:

$$
\begin{gathered}
H_{2}^{i}+H_{5}^{i}+H_{8}^{i}+\cdots+H_{(3 n-1)}^{i}=0 \\
H_{3}^{i}+H_{6}^{i}+H_{9}^{i}+\cdots+H_{(3 n)}^{i}=0
\end{gathered}
$$

As equações 2.115, 2.116 e 2.117 podem ser utilizadas para determinar indiretamente o valor dos termos das submatrizes $\mathbf{c}^{\mathrm{i}}$. Depois da obtenção da matriz $\hat{\mathbf{H}}$, percorre-se cada linha $i$ com as operações:

$$
\begin{aligned}
& \mathbf{R}_{1}^{\mathrm{i}}=\sum_{\mathbf{j}=1}^{\mathrm{n}}\left[\hat{\mathbf{H}}_{(3 \mathbf{j}-2)}^{\mathrm{i}}\right] \\
& \mathbf{R}_{2}^{\mathrm{i}}=\sum_{\mathbf{j}=1}^{\mathrm{n}}\left[\hat{\mathbf{H}}_{(3 \mathbf{j}-1)}^{\mathrm{i}}\right] \\
& \mathbf{R}_{3}^{\mathrm{i}}=\sum_{\mathbf{j}=1}^{\mathrm{n}}\left[\hat{\mathbf{H}}_{(3 \mathbf{j})}^{\mathrm{i}}\right]
\end{aligned}
$$

Os vetores $\mathbf{R}_{\mathbf{1}}^{\mathbf{i}}, \mathbf{R}_{\mathbf{2}}^{\mathbf{i}}$ e $\mathbf{R}_{\mathbf{3}}^{\mathbf{i}}$ contêm a soma dos termos de cada linha $i$ da matriz $\hat{\mathbf{H}}$, correspondentes aos movimentos de corpo rígido nas direções $x_{1}, x_{2}$ e $x_{3}$, respectivamente. Para obter a matriz final $\mathbf{H}$, utilizada na equação 2.110, basta subtrair os termos $\mathbf{R}_{\mathbf{1}}^{\mathbf{i}}, \mathbf{R}_{\mathbf{2}}^{\mathbf{i}}$ e $\mathbf{R}_{\mathbf{3}}^{\mathbf{i}}$ calculados para cada linha das posições correspondentes de cada linha. Assim, os três termos corretores da submatriz $\mathbf{c}^{\mathbf{i}}$ para uma linha $i$ qualquer de $\hat{\mathbf{H}}$ são:

$$
\begin{aligned}
& \mathbf{c}_{1}^{\mathrm{i}}=-\mathbf{R}_{1}^{\mathrm{i}} \\
& \mathbf{c}_{2}^{\mathrm{i}}=-\mathbf{R}_{2}^{\mathrm{i}} \\
& \mathbf{c}_{3}^{\mathrm{i}}=-\mathbf{R}_{3}^{\mathrm{i}}
\end{aligned}
$$

A posição na linha $i$ da matriz $\hat{\mathbf{H}}$ na qual cada termo de $\mathbf{c}^{\mathbf{i}}$ deve ser somado pode ser encontrada a partir do número $j$ do nó ao qual pertence o deslocamento em questão. Aplicando a regra imposta pela relação 2.108, conclui-se que o termo $\mathbf{c}_{1}^{\mathbf{i}}$ deve ser somado na posição $3 j-2$ da linha, o termo $\mathbf{c}_{2}^{\mathbf{i}}$ deve ser somado na posição $3 j-1$ e o termo $\mathbf{c}_{3}^{\mathbf{i}}$ deve ser somado na posição $3 j$.

Apesar de estar resolvido o problema da submatriz $\mathbf{c}^{\mathbf{i}}$, deve-se ressaltar que os deslocamentos segundo os três eixos coordenados não são os únicos movimentos de corpo rígido possíveis. Ainda podem ocorrer três rotações, uma para cada eixo. Assim, caso se tenha dúvida quanto à matriz $\mathbf{H}$ mesmo após a correção com as submatrizes $\mathbf{c}^{\mathbf{i}}$, ainda é possível conferir suas propriedades quanto aos movimentos de corpo rígido rotacionais. 


\subsubsection{Pontos internos}

Após determinar todos os valores de contorno é possível, a partir deles, determinar deslocamentos e tensões em qualquer ponto interno ao domínio $\Omega$ do sólido. Para obter uma equação cuja solução sejam os deslocamentos em pontos internos, primeiramente deve-se voltar à equação 2.73 com $b_{k}$ igual a zero, ou seja:

$$
u_{l}^{i}+\int_{\Gamma} u_{k} p_{l k}^{*} d \Gamma=\int_{\Gamma} p_{k} u_{l k}^{*} d \Gamma
$$

Para resolver a equação 2.124 para um ponto no contorno $\Gamma$ do sólido, foi necessário analisar a integral à direta da igualdade em 2.124 de forma especial, devido à singularidade no ponto. Porém, quando se considera um ponto interno ao domínio $\Omega$ do sólido, não há sigularidade porque o ponto fonte nunca coincide com o ponto campo. Assim, pode-se efetuar ambas as integrais da equação 2.124 sem se preocupar com singularidades. A partir da dedução feita para o ponto do contorno, aplicando os mesmos procedimentos numéricos entre as equações 2.73 e 2.106 na equação 2.124, pode-se deduzir que a equação resultante é a seguinte:

$$
u^{i}=\sum_{j=1}^{3 n} G_{i j} p^{j}-\sum_{j=1}^{3 n} H_{i j} u^{j}
$$

Na equação 2.125, $n$ corresponde ao número de nós, $i$ correponde ao número nó interno ou ponto campo e $j$ é o número do nó do contorno ou ponto fonte. A partir dela, pode-se determinar diretamente o deslocamento em qualquer ponto interno.

Além dos deslocamentos, também é possível determinar o tensor de tensões em um ponto interno $i$. Para isto, primeiramente é necessário encontrar a equação diferencial em deslocamentos que permite obter tensões no interior de um sólido elástico. Parte-se da relação 2.23 , que é:

$$
\varepsilon_{i j}=\frac{1}{2}\left(\frac{\partial u_{i}}{\partial x_{j}}+\frac{\partial u_{j}}{\partial x_{i}}\right)
$$

Outra expressão a ser utilizada é a seguinte relação constitutiva:

$$
\sigma_{i j}=\frac{E}{(1+\nu)}\left[\frac{\nu}{(1-2 \nu)} \delta_{i j} \varepsilon_{k k}+\varepsilon_{i j}\right]
$$

Substituindo a relação 2.126 na expressão 2.127, obtém-se a expressão:

$$
\sigma_{i j}=\frac{E}{(1+\nu)}\left[\frac{\nu}{(1-2 \nu)} \delta_{i j} u_{k, k}+\frac{1}{2}\left(u_{i, j}+u_{j, i}\right)\right]
$$


$\mathrm{Ou}$

$$
\sigma_{i j}=\frac{2 \nu G}{(1-2 \nu)} \delta_{i j} u_{l, l}+\left(u_{i, j}+u_{j, i}\right) G
$$

Isolando o deslocameto $\mathbf{u}^{\mathbf{i}}$ na equação 2.124 e substituindo em 2.129 , obtém-se:

$$
\begin{aligned}
\sigma_{i j} & =\int_{\Gamma}\left\{\frac{2 \nu G}{(1-2 \nu)} \delta_{i j} u_{l k, l}^{*}+\left(u_{i k, j}^{*}+u_{j k, i}^{*}\right) G\right\} p_{k} d \Gamma+ \\
& -\int_{\Gamma}\left\{\frac{2 \nu G}{(1-2 \nu)} \delta_{i j} p_{l k, l}^{*}+\left(p_{i k, j}^{*}+p_{j k, i}^{*}\right) G\right\} u_{k} d \Gamma
\end{aligned}
$$

Todas as derivadas da expressão 2.130 são tomadas no ponto interno, considerando onde está sendo aplicada a solução fundamental. Esta relação pode ser reescrita de forma mais compacta da seguinte maneira:

$$
\sigma_{i j}=\int_{\Gamma} D_{k i j} p_{k} d \Gamma-\int_{\Gamma} S_{k i j} u_{k} d \Gamma
$$

Em que os termos $D_{k i j}$ e $S_{k i j}$ são tensores. Estes tensores são dados por:

$$
\begin{gathered}
D_{k i j}=\frac{1}{8 \pi(1-\nu) r^{2}}\left\{(1-2 \nu)\left[\delta_{k i} r_{, j}+\delta_{k j} r_{, i}-\delta_{i j} r_{, k}\right]+3 r_{, k} r_{, i} r_{, j}\right\} \\
S_{k i j}=\frac{G}{4 \pi(1-\nu) r^{3}}\left\{3 r_{, \eta}\left[(1-2 \nu) \delta_{i j} r_{, k}+\nu\left(\delta_{i k} r_{, j}+\delta_{j k} r_{, i}\right)-5 r_{, k} r_{, i} r_{, j}\right]+\right. \\
\left.+3 \nu\left(\eta_{i} r_{, j} r_{, k}+\eta_{j} r_{, i} r_{, k}\right)+(1-2 \nu)\left(3 \eta_{k} r_{, i} r_{, j}+\eta_{j} \delta_{i k}+\eta_{i} \delta_{j k}\right)-(1-4 \nu) \eta_{k} \delta_{i j}\right\}
\end{gathered}
$$

\subsubsection{Subelementação}

Para melhorar a precisão dos resultados caso o ponto fonte esteja muito próximo ao elemento de contorno integrado, divide-se este elemento em áreas menores denominadas subelementos. Desta forma, para cada subelemento, pode-se definir mais pontos de integração. Percorre-se então todos os subelementos definidos no elemento e soma-se cada influência para obter a influência total do elemento.

No trabalho apresentado em ALMEIDA (2003a), dois tipos de subelementação são empregadas, convencional e progressiva. Na subelementação convencional o elemento é dividido de forma regular, conforme mostrado na figura 2.10.

O tamanho dos subelementos é definido a partir da distância do ponto fonte ao elemento em questão. Caso o ponto fonte esteja muito próximo, são definidos subelementos muito pequenos e em grande quantidade no elemento. Caso esteja distante o suficiente, é definido um único subelemento que computa toda a influência do elemento.

Caso seja feita subelementação progressiva, a divisão no elemento é mais intensa em sua região mais próxima do ponto fonte. Na figura 2.11, por exemplo, é mostrada uma possível configuração quando o ponto fonte se encontra próximo ao vértice $P$ 


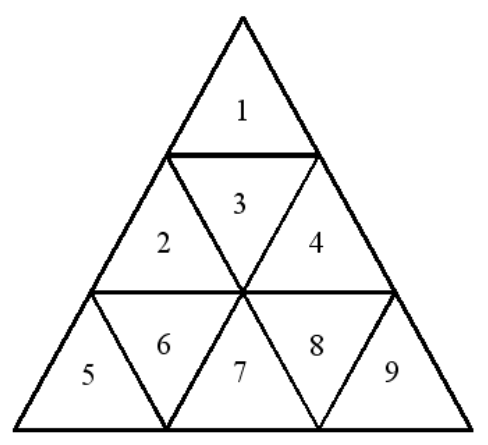

Figura 2.10: Subelementação convencional

do elemento de contorno.

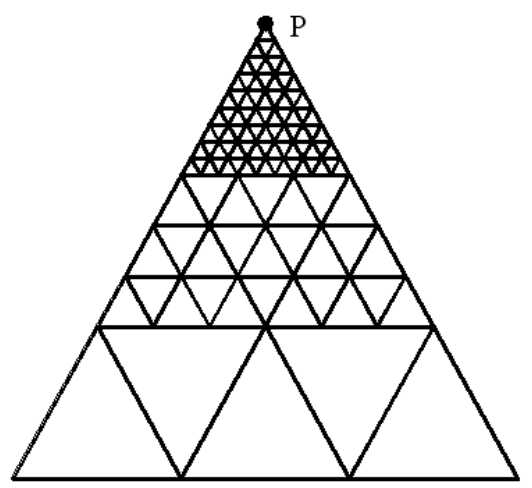

Figura 2.11: Subelementação progressiva

A vantagem da subelementação progressiva é que ela reduz o tamanho dos subelementos onde é mais necessário. Com isto, é possível obter resultados precisos e com menor tempo de processamento quando comparado com a subelementação convencional.

A subelementação demanda muito tempo de processamento, sendo a parte do programa que exige maior esforço computacional. Isto torna valiosa a utilização de subelementação progressiva, pois a diferença de tempo é significativa. O programa computacional utilizado neste trabalho contém os dois tipos, podendo-se optar entre um e outro.

\subsection{Considerações finais}

Neste capítulo, a partir de equações advindas da mecânica dos sólidos e de técnicas de resíduos ponderados, deduziu-se a equação integral de contorno denominada Identidade Somigliana. Apresentou-se também as soluções fundamentais de Kelvin de deslocamento e força para uma carga unitária aplicada em um meio infinito. A partir da Identidade Somigliana e das soluções fundamentais, formulou-se o problema do valor de contorno e foi obtida a equação 2.80, que é o ponto de partida do 
MEC.

Adotando aproximações lineares para deslocamentos e forças, foi obtido um sistema de equações cuja solução leva aos valores incógnitos nos pontos definidos no contorno. Descreveu-se então o cálculo de deslocamentos e tensões em pontos internos do sólido a partir dos valores de contorno.

Ao final do capítulo, foram abordados os tipos de subelementação contidos no programa utilizado neste trabalho. Concluiu-se que a subelementação progressiva, por produzir subelementos menores onde é mais necessário, é mais eficiente que a convencional e leva a resultados mais precisos. 


\section{Capítulo 3}

\section{O Método dos Elementos Finitos}

\subsection{Introdução}

O Método dos Elementos Finitos (MEF) é uma ferramenta numérica utilizada na resolução de diversos problemas de engenharia. Neste trabalho esta ferramenta é empregada na simulação de estruturas compostas por lâminas e retículas, em análise estática, elástica e linear. A teoria é apresentada de forma sucinta, referenciando publicações complementares.

A partir do equacionamento básico do problema elástico, obtém-se uma equação diferencial que representa o equilíbrio de um sólido qualquer. Aplicando-se nesta equação técnicas de resíduos ponderados, chega-se a uma expressão que representa os trabalhos interno e externo associados à estrutura em estudo. Divide-se então a estrutura em um número qualquer de subdomínios, denominados elementos finitos. A cada elemento é aplicada a equação dos trabalhos interno e externo, adotando aproximações para os deslocamentos e deformações. O resultado é um sistema de equações para cada elemento, tornando-se possível então montar um único sistema válido para toda a estrutura. Após considerar as condições de contorno do problema é possível resolver este sistema, obtendo-se deslocamentos em pontos definidos na estrutura em questão.

Este mesmo tema pode ser encontrado em ASSAN (2003).

\subsection{O Princípio dos Trabalhos Virtuais (PTV)}

O MEF é, de forma geral, a mais poderosa e eficiente ferramenta para análise de problemas de domínio finito e de geometria qualquer. Neste trabalho o MEF é aplicado utilizando-se o Método dos Deslocamentos, o que implica em aproximar o campo de deslocamentos de cada elemento finito por funções ponderadoras. Estas funções estão relacionadas aos parâmetros nodais do elemento, que são valores de 
deslocamento nos nós do elemento.

No capítulo2 foi descrito sucintamente o problema elástico, e foi obtida a seguinte equação de equilíbrio:

$$
\sigma_{i j, j}+b_{i}=0
$$

Nesta equação, $\sigma_{i j}$ são as componentes de tensão em um ponto qualquer de um sólido tridimensional submetido a cargas externas $b_{i}$. Para equacionar o PTV considera-se a equação 3.1 válida em um sólido tridimensional qualquer, conforme ilustrado na figura 3.1. A este sólido são impostas condições de contorno essenciais e naturais arbitrárias.
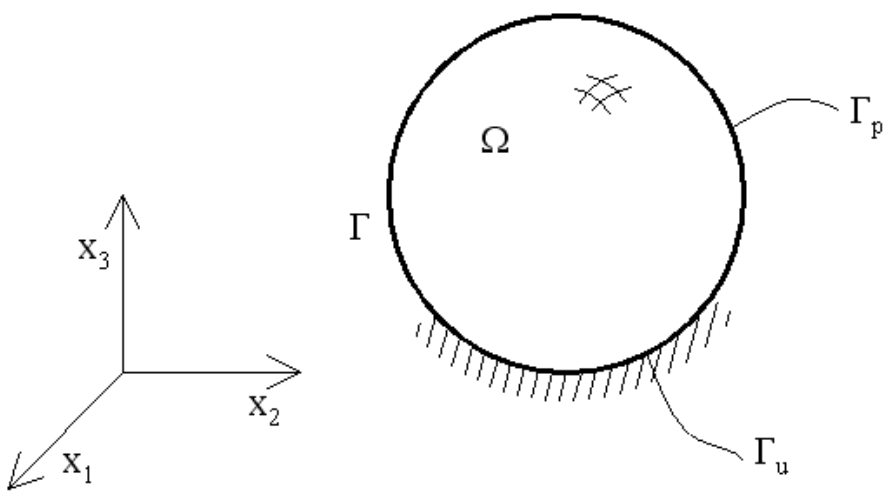

Figura 3.1: Sólido qualquer com condições de contorno arbitrárias

Na figura 3.1. $\Omega$ é o domínio do sólido e $\Gamma$ é seu contorno. As condições de contorno essenciais ou em deslocamento estão aplicadas na parcela $\Gamma_{u}$ do contorno $\Gamma$ do sólido. Isto é, em $\Gamma_{u}$ :

$$
u_{i}=\bar{u}_{i}
$$

sendo $i$ uma direção do sistema $x_{1} x_{2} x_{3}$.

Por outro lado, as condições de contorno naturais ou em força estão aplicadas no trecho $\Gamma_{p}$ do contorno. Ou seja, em $\Gamma_{p}$ :

$$
p_{i}=\bar{p}_{i}
$$

A soma dos trechos $\Gamma_{u}$ e $\Gamma_{p}$ compõe o contorno total $\Gamma$ do sólido, ou seja:

$$
\Gamma=\Gamma_{u}+\Gamma_{p}
$$

Definido o problema em questão, a equação 3.1 pode ser trabalhada aplicando-se técnicas de resíduos ponderados. A função ponderadora a ser escolhida corresponde a um campo de deslocamentos virtuais $\tilde{u}_{i}$, o qual deve satisfazer as condições de contorno essenciais impostas ao problema. Para simplificar, considera-se que no 
contorno $\Gamma_{u}$ prescreveu-se deslocamentos iguais a zero. Ou seja, em $\Gamma_{u}$ :

$$
\tilde{u}_{i}=0
$$

Definida a função ponderadora, escreve-se a expressão:

$$
\int_{\Omega}\left(\sigma_{i j, j}+b_{i}\right) \tilde{u}_{i} d \Omega=0
$$

Integrando a expressão 3.6 por partes, obtém-se:

$$
-\int_{\Omega} \sigma_{i j} \tilde{u}_{i, j} d \Omega+\int_{\Omega} b_{i} \tilde{u}_{i} d \Omega+\int_{\Gamma} p_{i} \tilde{u}_{i} d \Gamma=0
$$

A expressão 3.7, escrita em forma matricial, é equivalente a:

$$
\int_{\Omega}[\tilde{\varepsilon}]^{\mathbf{T}}[\sigma] \mathbf{d} \Omega=\int_{\Omega}\{\tilde{\mathbf{u}}\}^{\mathbf{T}}\{\mathbf{b}\} \mathbf{d} \boldsymbol{\Omega}+\int_{\Gamma}\{\tilde{\mathbf{u}}\}^{\mathbf{T}}\{\mathbf{p}\} \mathbf{d} \boldsymbol{\Gamma}
$$

em que $\tilde{\varepsilon}^{\mathbf{T}}$ corresponde ao campo de deformações virtuais decorrentes do campo de deslocamentos virtuais $\tilde{\mathbf{u}}$. Considerando que as condições de contorno prescritas em deslocamento são iguais a zero, a segunda integral à direita da equação 3.8 fica restrita ao contorno $\boldsymbol{\Gamma}_{\mathbf{p}}$. Assim, chega-se à equação integral:

$$
\int_{\boldsymbol{\Omega}}[\tilde{\varepsilon}]^{\mathbf{T}}[\sigma] \mathbf{d} \boldsymbol{\Omega}=\int_{\boldsymbol{\Omega}}\{\tilde{\mathbf{u}}\}^{\mathbf{T}}\{\mathbf{b}\} \mathbf{d} \boldsymbol{\Omega}+\int_{\boldsymbol{\Gamma}_{\mathbf{p}}}\{\tilde{\mathbf{u}}\}^{\mathbf{T}}\{\overline{\mathbf{p}}\} \mathbf{d} \boldsymbol{\Gamma}
$$

em que $\overline{\mathbf{p}}$ são as condições de contorno naturais do problema.

A integral à esquerda da equação 3.9 é o trabalho virtual interno mobilizado no sólido, enquanto as integrais à direita são o trabalho virtual externo decorrente das cargas externas aplicadas no sólido. O trabalho interno, que está diretamente ligado aos esforços internos estabelecidos no sólido, está equacionado em função das tensões $\sigma$ atuantes no sólido. Para dar continuidade às deduções, é preciso expressar esta integral em função das deformações reais $\varepsilon$ correspondentes às tensões $\sigma$. Pode-se relacionar o campo de tensões ao de deformações por meio de relações constitutivas. Estas relações podem ser representadas matricialmente pela relação:

$$
[\sigma]=[\mathbf{D}][\varepsilon]
$$

A matriz D representa um tensor de quarta ordem que traduz as características do material. Uma das hipóteses iniciais deste trabalho é considerar o material elástico, linear e homogêneo, e por isto a matriz D é constante. 
Substituindo a relação 3.10 na expressão 3.9 , obtém-se:

$$
\int_{\boldsymbol{\Omega}}[\tilde{\varepsilon}]^{\mathbf{T}}[\mathbf{D}][\varepsilon] \mathbf{d} \boldsymbol{\Omega}=\int_{\boldsymbol{\Omega}}\{\tilde{\mathbf{u}}\}^{\mathbf{T}}\{\mathbf{b}\} \mathbf{d} \boldsymbol{\Omega}+\int_{\boldsymbol{\Gamma}_{\mathbf{p}}}\{\tilde{\mathbf{u}}\}^{\mathbf{T}}\{\overline{\mathbf{p}}\} \mathbf{d} \boldsymbol{\Gamma}
$$

Na expressão 3.11, a função ponderadora ũ pode ser qualquer uma que satisfaça as condições de contorno essenciais do problema. Pelo Método de Galerkin, descrito em ASSAN (2003), esta função é o próprio campo de deslocamentos real u do problema. Assim, adotando este procedimento, a expressão 3.11 se torna:

$$
\int_{\boldsymbol{\Omega}}[\varepsilon]^{\mathbf{T}}[\mathbf{D}][\varepsilon] \mathbf{d} \boldsymbol{\Omega}=\int_{\boldsymbol{\Omega}}\{\mathbf{u}\}^{\mathbf{T}}\{\mathbf{b}\} \mathbf{d} \boldsymbol{\Omega}+\int_{\boldsymbol{\Gamma}_{\mathbf{p}}}\{\mathbf{u}\}^{\mathbf{T}}\{\overline{\mathbf{p}}\} \mathbf{d} \boldsymbol{\Gamma}
$$

A equação 3.12 representa o Princípio dos Trabalhos Virtuais (PTV). Para resolver esta equação pelo MEF, deve-se dividir o domínio $\Omega$ do sólido em um determinado número de subdomínios $\boldsymbol{\Omega}_{\mathbf{e}}$, denominados elementos finitos. A equação 3.12 é então aplicada nestes subdomínios, e a influência de todos eles é somada posteriormente. Considerando um elemento finito qualquer, escreve-se para ele a expressão 3.12 como:

$$
\int_{\boldsymbol{\Omega}_{\mathbf{e}}}[\varepsilon]^{\mathbf{T}}[\mathbf{D}][\varepsilon] \mathbf{d} \boldsymbol{\Omega}=\int_{\boldsymbol{\Omega}_{\mathbf{e}}}\{\mathbf{u}\}^{\mathbf{T}}\{\mathbf{b}\} \mathbf{d} \boldsymbol{\Omega}+\int_{\Gamma_{\mathbf{e}}}\{\mathbf{u}\}^{\mathbf{T}}\{\overline{\mathbf{p}}\} \mathrm{d} \boldsymbol{\Gamma}
$$

em que $\boldsymbol{\Gamma}_{\mathbf{e}}$ é o contorno do elemento finito em questão.

As integrais em 3.13 são calculadas empregando funções interpoladoras que aproximam o campo de deslocamentos u e o campo de deformações $\varepsilon$ em função dos parâmetros nodais do elemento. Estes parâmetros são os valores de deslocamento nos nós do elemento. Desta forma, para o deslocamento u, escreve-se:

$$
\{\mathbf{u}\}=\left[\mathbf{H}_{\left(\mathbf{x}_{\mathbf{i}}\right)}\right]\left\{\mathbf{u}^{\text {nodal }}\right\}
$$

Na igualdade 3.14, $\mathbf{x}_{\mathbf{i}}$ são coordenadas locais definidas em cada elemento finito, $\mathbf{H}$ é uma matriz de funções interpoladoras de deslocamento conhecidas e $\mathbf{u}^{\text {nodal }}$ é um vetor que contém os valores de deslocamento nos nós do elemento. Da mesma forma, para o campo de deformações $\varepsilon$ :

$$
\{\varepsilon\}=\left[\mathbf{B}_{\left(\mathbf{x}_{\mathbf{i}}\right)}\right]\left\{\mathbf{u}^{\text {nodal }}\right\}
$$

em que B é uma matriz de funções interpoladoras de deformação. Substituindo as 
relações 3.14 e 3.15 em 3.13 , chega-se à expressão:

$$
\begin{gathered}
\left\{\mathbf{u}^{\text {nodal }}\right\}^{\mathbf{T}}\left(\int_{\Omega_{\mathrm{e}}}[\mathbf{B}]^{\mathbf{T}}[\mathbf{D}][\mathbf{B}] \mathbf{d} \Omega\right)\left\{\mathbf{u}^{\text {nodal }}\right\}=\left\{\mathbf{u}^{\text {nodal }}\right\}^{\mathbf{T}} \int_{\Omega_{\mathrm{e}}}[\mathbf{H}]^{\mathbf{T}}\{\mathrm{b}\} \mathrm{d} \Omega+ \\
+\left\{\mathbf{u}^{\text {nodal }}\right\}^{\mathbf{T}} \int_{\Gamma_{\mathbf{e}}}[\mathbf{H}]^{\mathbf{T}}\{\overline{\mathbf{p}}\} \mathbf{d} \boldsymbol{\Gamma}
\end{gathered}
$$

Após resolver as integrais, a equação 3.16 resulta em um sistema de equações escritas para o elemento finito em questão. Este sistema é:

$$
\left[\mathbf{K}_{\mathbf{e}}\right]\left\{\mathbf{u}^{\text {nodal }}\right\}=\left\{\mathbf{f}_{\mathbf{e}}\right\}
$$

em que:

$$
\left[\mathbf{K}_{\mathbf{e}}\right]=\left(\int_{\Omega_{\mathbf{e}}}[\mathbf{B}]^{\mathbf{T}}[\mathbf{D}][\mathbf{B}] \mathbf{d} \Omega\right)
$$

$\mathrm{e}$

$$
\left\{\mathbf{f}_{\mathbf{e}}\right\}=\int_{\boldsymbol{\Omega}_{\mathbf{e}}}[\mathbf{H}]^{\mathbf{T}}\{\mathbf{b}\} \mathbf{d} \boldsymbol{\Omega}+\int_{\Gamma_{\mathbf{e}}}[\mathbf{H}]^{\mathbf{T}}\{\overline{\mathbf{p}}\} \mathbf{d} \boldsymbol{\Gamma}
$$

O termo $\mathbf{K}_{\mathbf{e}}$ é a matriz de rigidez do elemento finito e o termo $\mathbf{f}_{\mathbf{e}}$ é o vetor de cargas nodais do elemento. Escrevendo a equação 3.17 para todos os elementos finitos definidos no sólido, é possível montar uma única matriz de rigidez que guarda a influência de todos os elementos, assim como um único vetor de cargas nodais. Para isto, deve-se rotacionar as matrizes $\mathbf{K}_{\mathbf{e}}$ e os vetores $\mathbf{f}_{\mathbf{e}}$ do sistema de coordenadas local $\mathbf{x}_{\mathbf{i}}^{1}$ para o global $\mathbf{x}_{\mathbf{i}}$. A matriz de rigidez global com o vetor de cargas nodais global compõem um sistema de equações do tipo:

$$
[\mathbf{K}]\{\mathbf{u}\}=\{\mathbf{f}\}
$$

Após aplicar as condições de contorno, este sistema pode ser resolvido e sua solução resulta em valores de deslocamento nos nós de todos os elementos.

\subsection{Elementos finitos laminares}

Nesta seção é feita uma breve descrição da formulação empregada nos elementos finitos laminares que são utilizados na superestrutura do programa utilizado neste trabalho. A combinação destes elementos permite a simulação de uma grande variedade de estruturas, como por exemplo silos e galpões. Esta abrangência se dá pela da combinação do elemento de placa DKT com um elemento de membrana, como será visto mais adiante.

Podem ser considerados laminares estruturas tridimensionais nas quais uma dimensão é muito menor que as outras duas. São exemplos disto placas, paredes e 
estruturas em geral formadas por estes dois subsistemas.

Na formulação utilizada neste trabalho, são consideradas as hipóteses de KirchoffLove. Estas são:

- A espessura da lâmina é pequena quando comparada às suas demais dimensões e aos raios de curvatura de sua superfície média.

- As tensões normais à superfície média são desprezíveis em relação às demais.

- Um ponto pertencente a uma reta ortogonal ao plano médio indeformado, após a lâmina ter se deformado continua pertencendo à mesma reta ortogonal ao plano médio deformado.

- Os deslocamentos normais ao plano médio são pequenos quando comparados à espessura da lâmina.

Partindo destas hipóteses, o campo de deslocamentos em um elemento finito laminar pode ser escrito como:

$$
\{\mathbf{u}\}=\left\{\begin{array}{c}
u\left(x_{1}, x_{2}, x_{3}\right) \\
v\left(x_{1}, x_{2}, x_{3}\right) \\
w\left(x_{1}, x_{2}, x_{3}\right)
\end{array}\right\}=\left\{\begin{array}{c}
u_{0}\left(x_{1}, x_{2}\right)-x_{3} \frac{\partial w_{0}}{\partial x_{1}} \\
v_{0}\left(x_{1}, x_{2}\right)-x_{3} \frac{\partial w_{0}}{\partial x_{2}} \\
w_{0}\left(x_{1}, x_{2}\right)
\end{array}\right\}
$$

Na igualdade 3.21, os deslocamentos $u$ e $u_{0}$ encontram-se na direção do eixo $x_{1}$, os deslocamentos $v$ e $v_{0}$ estão na direção do eixo $x_{2}$ e os deslocamentos $w$ e $w_{0}$ estão na direção do eixo $x_{3}$. Os deslocamentos $u, v$ e $w$ se referem a um ponto $P$ qualquer no domínio da placa, e os deslocamentos $u_{0}, v_{0}$ e $w_{0}$ ocorrem na projeção $P^{\prime}$ do ponto $P$ no plano médio da placa deformada. Estas informações podem ser visualizadas na figura 3.2 .
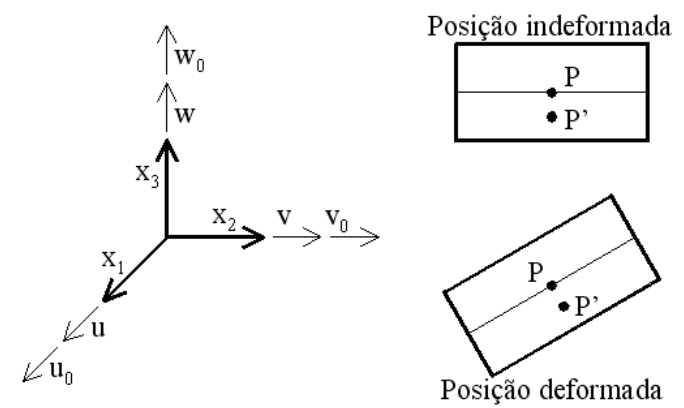

Figura 3.2: Direção dos deslocamentos e posição dos pontos $P$ e $P^{\prime}$

Ao determinar o campo de deformações, deve-se separar as parcelas referentes 
ao efeito da flexão e ao efeito de membrana. As deformações são dadas então por:

$$
\{\varepsilon\}=\{\varepsilon\}_{\mathbf{m}}+\{\varepsilon\}_{\mathbf{f}}=\left\{\begin{array}{c}
\frac{\partial u_{0}}{\partial x_{1}} \\
\frac{\partial v_{0}}{\partial x_{2}} \\
\frac{\partial u_{0}}{\partial x_{2}}+\frac{\partial v_{0}}{\partial x_{1}}
\end{array}\right\}-x_{3}\left\{\begin{array}{c}
\frac{\partial^{2} w_{0}}{\partial x_{1}^{2}} \\
\frac{\partial^{2} w_{0}}{\partial x_{2}^{2}} \\
2 \frac{\partial^{2} w_{0}}{\partial x_{1} \partial x_{2}}
\end{array}\right\}
$$

$\mathrm{Na}$ igualdade 3.22 , o índice subscrito $\mathbf{m}$ indica efeito de membrana e o índice subscrito $\mathbf{f}$ indica efeito de flexão.

Para determinar a matriz de rigidez do elemento considerado, deve-se determinar as matrizes $\mathbf{B}$ e $\mathbf{D}$ que aparecem na equação 3.16. A obtenção de $\mathbf{D}$ parte da aplicação das relações constitutivas entre tensão e deformação. Ou seja:

$$
[\sigma]=[\mathbf{D}][\varepsilon]
$$

A obtenção da matriz $\mathbf{B}$ envolve as aproximações adotadas para os deslocamentos no elemento. Estas são dependentes dos parâmetros nodais do elemento, e podem ser escritas como:

$$
\{\mathbf{u}\}=[\varphi]\left\{\mathbf{u}^{\text {nodal }}\right\}=\left[\begin{array}{cc}
{\left[\varphi_{\mathbf{m}}\right]} & {[\mathbf{0}]} \\
{[\mathbf{0}]} & {\left[\varphi_{\mathbf{f}}\right]}
\end{array}\right]\left\{\begin{array}{l}
\mathbf{u}_{\mathbf{m}}^{\text {nodal }} \\
\mathbf{u}_{\mathbf{f}}^{\text {nodal }}
\end{array}\right\}
$$

O subvetor $\mathbf{u}_{\mathbf{m}}^{\text {nodal }}$ contém todos os graus de liberdade do elemento finito referentes ao efeito de membrana e o subvetor $\mathbf{u}_{\mathbf{f}}^{\text {nodal }}$ contém todos os graus de liberdade referentes ao efeito de flexão. As submatrizes $\varphi_{\mathbf{f}}$ e $\varphi_{\mathbf{m}}$ contêm as funções de forma adotadas para a parcela de flexão e de membrana, respectivamente. A aproximação para as deformações, considerando a relação 3.22 , é:

$$
\{\varepsilon\}=[\mathbf{B}]\left\{\mathbf{u}^{\text {nodal }}\right\}=\left[\left[\mathbf{B}_{\mathbf{m}}\right] \quad \mathbf{x}_{\mathbf{3}}\left[\mathbf{B}_{\mathbf{f}}\right]\right]\left\{\mathbf{u}^{\text {nodal }}\right\}
$$

O termo $\mathbf{B}_{\mathbf{m}}$ é a matriz que contém as funções de interpolação referentes ao efeito de membrana e o termo $\mathbf{B}_{\mathbf{f}}$ é a matriz que contém as funções de interpolação referentes ao efeito de flexão. Substituindo as matrizes B e D na expressão 3.18 , pode-se obter a matriz de rigidez do elemento laminar pela expressão:

$$
\left[\mathbf{K}_{\mathbf{e}}\right]=\int_{\Omega_{\mathbf{e}}}[\mathbf{B}]^{\mathbf{T}}[\mathbf{D}][\mathbf{B}] \mathbf{d} \Omega
$$

em que $\Omega_{\mathbf{e}}$ é o domínio do elemento. Expandindo as matrizes $\mathbf{B}$ e $\mathbf{D}$, a expressão 3.26 se torna:

$$
\left[\mathbf{K}_{\mathbf{e}}\right]=\int_{\boldsymbol{\Omega}_{\mathbf{e}}}\left[\begin{array}{c}
{\left[\mathbf{B}_{\mathbf{m}}\right]^{\mathbf{T}}} \\
x_{3}\left[\mathbf{B}_{\mathbf{f}}\right]^{\mathbf{T}}
\end{array}\right]\left[\begin{array}{cc}
{\left[\mathbf{D}_{\mathbf{m}}\right]} & {\left[\mathbf{D}_{\mathbf{m f}}\right]} \\
{\left[\mathbf{D}_{\mathbf{f m}}\right]} & {\left[\mathbf{D}_{\mathbf{f}}\right]}
\end{array}\right]\left[\left[\mathbf{B}_{\mathbf{m}}\right] x_{3}\left[\mathbf{B}_{\mathbf{f}}\right]\right] \mathbf{d} \boldsymbol{\Omega}
$$


Após efetuar a integral, obtém-se:

$$
\left[\mathbf{K}_{\mathbf{e}}\right]=\left[\begin{array}{cc}
{\left[\mathbf{K}_{\mathbf{m}}\right]} & {[\mathbf{0}]} \\
{[\mathbf{0}]} & {\left[\mathbf{K}_{\mathbf{f}}\right]}
\end{array}\right]
$$

As submatrizes de zeros ocorrem devido ao seguinte fator:

$$
\int_{-h / 2}^{h / 2} x_{3}[\mathbf{D}] \partial x_{3}=[\mathbf{0}]
$$

Assim, pode-se concluir que o termo $\mathbf{K}_{\mathbf{m f}}$ da relação 3.28 é nulo. Como a matriz é simétrica, o termo $\mathbf{K}_{\mathbf{f m}}$ também é nulo. Por causa destas propriedades da matriz $\mathbf{K}$, é possível afirmar que o efeito de membrana é independente do efeito de flexão nesta formulação.

\subsection{Graus de liberdade do elemento finito laminar}

Os elementos finitos laminares utilizados neste trabalho são triangulares e com três nós, havendo um nó em cada vértice. Dos graus de liberdade associados a cada nó, três são referentes ao elemento finito de membrana, FF, e três são referentes ao elemento finito de flexão, DKT. O elemento finito de membrana com os graus de liberdade de seus nós pode ser visualizado na figura 3.3 .
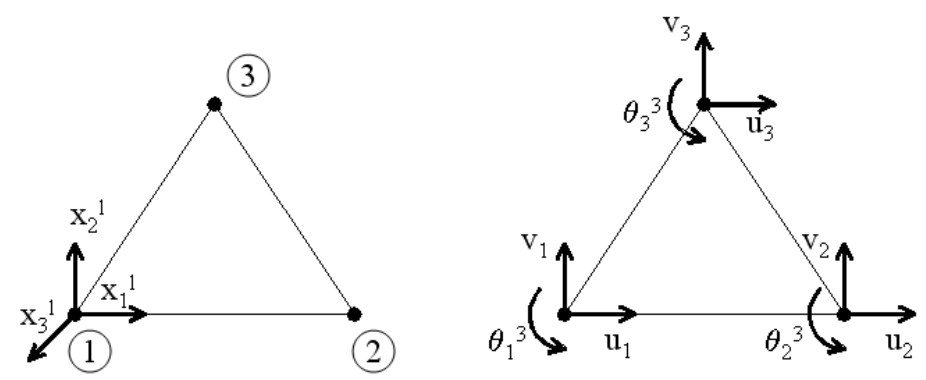

Figura 3.3: Graus de liberdade do elemento finito de membrana FF

Na figura 3.3 está ilustrado o sistema de coordenadas local $x_{i}^{l}$, a numeração dos nós e os graus de liberdade de cada nó referentes ao elemento finito de membrana. No sistema local, a origem está localizada no nó número 1 do elemento. O eixo $x_{1}^{l}$ é alinhado com o lado do elemento cujas extremidades são os nós 1 e 2, apontando para o nó 2. O eixo $x_{2}^{l}$ é perpendicular ao eixo $x_{1}^{l}$, e pertence ao plano do elemento. Por fim, o eixo $x_{3}^{l}$ é ortogonal ao plano do elemento.

Em cada nó do elemento de membrana existem três graus de liberdade. O deslocamento $u$ ocorre na direção do eixo local $x_{1}^{l}$ e o deslocamento $v$ ocorre na direção do eixo local $x_{2}^{l}$. A rotação $\theta$ se dá em torno do eixo $x_{3}^{l}$, sendo positiva do 
eixo $x_{1}^{l}$ para o eixo $x_{2}^{l}$. Os graus de liberdade nos nós do elemento organizados em forma de vetor são:

$$
\left\{\mathbf{u}_{\mathbf{m}}\right\}^{\mathbf{T}}=\left\{\begin{array}{lllllllll}
u_{1} & v_{1} & \theta_{1}^{3} & u_{2} & v_{2} & \theta_{2}^{3} & u_{3} & v_{3} & \theta_{3}^{3}
\end{array}\right\}
$$

O elemento finito utilizado neste trabalho para computar o efeito da flexão foi o DKT, como mencionado anteriormente. Este elemento com seus graus de liberdade está ilustrado na figura 3.4
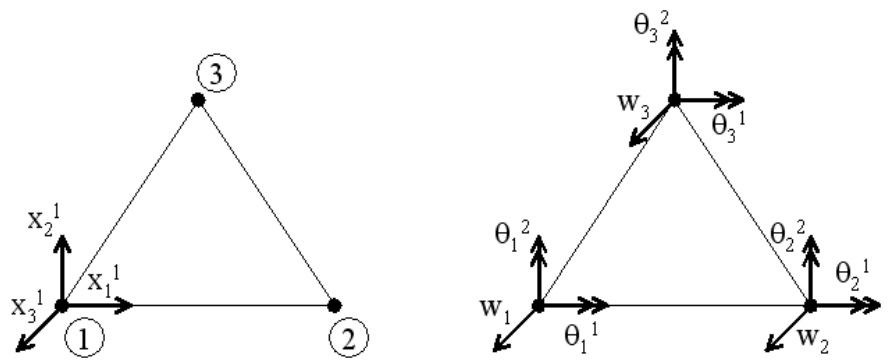

Figura 3.4: Graus de liberdade do elemento finito DKT

Nas rotações $\theta$, indicadas nos nós do elemento DKT na figura 3.4, o índice subscrito indica o número do nó e o índice sobrescrito indica o número do eixo local em torno do qual se dá a rotação. Os deslocamentos $w$ são na direção do eixo local $x_{3}^{l}$. Este graus de liberdade podem ser listados em um vetor como segue:

$$
\left\{\mathbf{u}_{\mathbf{f}}\right\}^{\mathbf{T}}=\left\{\begin{array}{lllllllll}
w_{1} & \theta_{1}^{1} & \theta_{1}^{2} & w_{2} & \theta_{2}^{1} & \theta_{2}^{2} & w_{3} & \theta_{3}^{1} & \theta_{3}^{2}
\end{array}\right\}
$$

O elemento finito de membrana indicado na figura 3.3 em conjunto com o elemento finito DKT indicado na figura 3.4 compõem o elemento finito laminar DKT/FF, que é utilizado neste trabalho. Este elemento com todos os seus graus de liberdade está ilustrado na figura 3.5 .
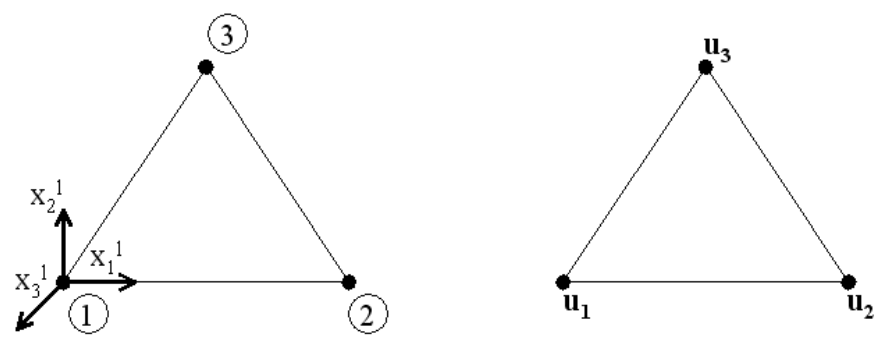

Figura 3.5: Graus de liberdade do elemento finito laminar DKT/FF

Os deslocamentos $\mathbf{u}$ indicados na figura 3.5 são vetores que contêm os graus de liberdade de cada nó. Estes vetores são escritos a seguir:

$$
\left\{\mathbf{u}_{1}\right\}^{\mathbf{T}}=\left\{\begin{array}{llllll}
u_{1} & v_{1} & \theta_{1}^{3} & w_{1} & \theta_{1}^{1} & \theta_{1}^{2}
\end{array}\right\}
$$




$$
\begin{aligned}
& \left\{\mathbf{u}_{2}\right\}^{\mathbf{T}}=\left\{\begin{array}{llllll}
u_{2} & v_{2} & \theta_{2}^{3} & w_{2} & \theta_{2}^{1} & \theta_{2}^{2}
\end{array}\right\} \\
& \left\{\mathbf{u}_{3}\right\}^{\mathbf{T}}=\left\{\begin{array}{llllll}
u_{3} & v_{3} & \theta_{3}^{3} & w_{3} & \theta_{3}^{1} & \theta_{3}^{2}
\end{array}\right\}
\end{aligned}
$$

São seis graus de liberdade por nó, totalizando os dezoito graus de liberdade do elemento finito DKT/FF.

\subsection{Rotação de eixos}

A matriz de rigidez do elemento finito $\mathrm{DKT} / \mathrm{FF}$ pode ser obtida pela expressão 3.28, a partir das submatrizes $\mathbf{K}_{\mathbf{m}}$ e $\mathbf{K}_{\mathbf{f}}$. A teoria envolvida na obtenção destas matrizes é extensa e expô-la neste texto desviaria muito dos objetivos deste trabalho. O desenvolvimento para a obtenção da matriz $\mathbf{K}_{\mathbf{m}}$ pode ser encontrado em BERGAN \& FELIPPA (1985), onde é utilizada a formulação livre. As deduções para a obtenção da matriz $\mathbf{K}_{\mathbf{f}}$, referentes ao elemento DKT, podem ser encontradas em BATOZ (1980).

Após deduzir a matriz de rigidez do elemento DKT/FF, é preciso definir como esta matriz pode ser rotacionada do sistema de coordenadas local para o global. Isto é necessário porque a matriz definida para cada elemento finito da estrutura deve ser rotacionada antes de computar sua influência na matriz de rigidez global da estrutura. Para formular este problema, considera-se um elemento finito genérico orientado segundo uma direção qualquer, conforme mostrado na figura 3.6 .

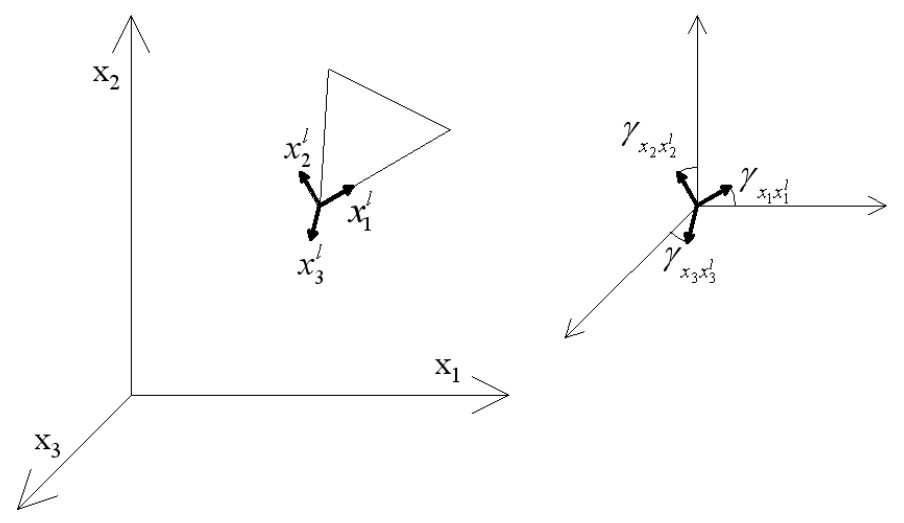

Figura 3.6: Sistema de coordenadas global $x_{1} x_{2} x_{3}$ e local $x_{1}^{l} x_{2}^{l} x_{3}^{l}$

O sistema de coordenadas local $x_{1}^{l} x_{2}^{l} x_{3}^{l}$ pode ser relacionado ao sistema de coordenadas global $x_{1} x_{2} x_{3}$ por meio de uma matriz de rotação $\bar{\beta}$. Assim, escreve-se a igualdade:

$$
\left\{\mathbf{x}_{\mathbf{i}}^{1}\right\}=[\bar{\beta}]\left\{\mathbf{x}_{\mathbf{i}}\right\}
$$


$\mathrm{Ou}$

$$
\left\{\begin{array}{l}
x_{1}^{l} \\
x_{2}^{l} \\
x_{3}^{l}
\end{array}\right\}=[\bar{\beta}]\left\{\begin{array}{l}
x_{1} \\
x_{2} \\
x_{3}
\end{array}\right\}
$$

A matriz de rotação $\bar{\beta}$ é dada por:

$$
[\bar{\beta}]=\left[\begin{array}{lll}
\gamma_{x_{1} x_{1}^{l}} & \gamma_{x_{1} x_{2}^{l}} & \gamma_{x_{1} x_{3}^{l}} \\
\gamma_{x_{2} x_{1}^{l}} & \gamma_{x_{2} x_{2}^{l}} & \gamma_{x_{2} x_{3}^{l}} \\
\gamma_{x_{3} x_{1}^{l}} & \gamma_{x_{3} x_{2}^{l}} & \gamma_{x_{3} x_{3}^{l}}
\end{array}\right]
$$

em que os temos $\gamma$ correspondem aos co-senos diretores entre os eixos locais e globais. Após determinar a matriz de rotação $\bar{\beta}$, é possível determinar a matriz a ser utlizada para rotacionar a matriz de rigidez do elemento DKT/FF. Esta matriz é:

$$
[\beta]=\left[\begin{array}{ll}
{[\bar{\beta}]} & {[\mathbf{0}]} \\
{[\mathbf{0}]} & {[\bar{\beta}]}
\end{array}\right]
$$

A partir da matriz 3.38 , pode-se rotacionar a matriz do elemento aplicando a expressão:

$$
\left[\overline{\mathbf{K}}_{\mathbf{e}}\right]=[\beta]^{\mathbf{T}}\left[\mathbf{K}_{\mathbf{e}}\right][\beta]
$$

A matriz $\overline{\mathbf{K}}_{\mathbf{e}}$ calculada pela expressão 3.39 para cada elemento, está pronta para ser computada na matriz de rigidez global da estrutura.

\subsection{Elementos utilizados no edifício}

No trabalho desenvolvido em ALMEIDA (2003a) foram utilizados dois tipos de elementos estruturais para compor a estrutura do edifício. O primeiro, usado para representar as vigas e pilares, é um elemento finito reticular no espaço tridimensional que não considera o efeito da torção. O segundo foi um diafragma rígido. A teoria envolvida na formulação destes subsistemas pode ser encontrada nas referências RIOS (1991) e BEZERRA (1995). A lâmina composta por elementos DKT/FF podia ser incluída como elemento de fundação para este edifício, sendo necessário ligar os nós dos pilares do andar térreo aos nós desta lâmina.

O fato de considerar as lajes rígidas resulta em deslocamentos e giros horizontais iguais para todos os nós definidos em cada pavimento. Por isto, na formulação empregada em ALMEIDA (2003a), a influência de todas as vigas e pilares são transferidas para um nó mestre, localizado no centro de torção do pavimento-tipo. As ações horizontais foram então prescritas pontualmente neste nó mestre, podendo ser forças ou momentos. Esta formulação foi modificada ao longo deste projeto. 
O elemento DKT/FF é agora também empregado nas lajes, discretizando-as em elementos finitos triangulares com seis graus de liberdade por nó. A formulação dos elementos reticulares também é diferente, estando agora definidos seis graus de liberdade nos nós de suas extremidades, ou seja, considerando também o efeito da torção.

\subsection{Considerações finais}

Neste capítulo, foi apresentada de forma sucinta a formulação do Método dos Elementos Finitos (MEF) empregada na simulação da superestrutura formada por lâminas e retículas, em análise elástica e linear.

Aplicando a teoria advinda da Mecânica dos Sólidos, foi obtida uma equação de equilíbrio válida para um sólido qualquer. A partir desta equação e de técnicas de resíduos ponderados, foi possível obter uma expressão que representa os trabalhos externo e interno, referentes às cargas externas e aos esforços internos, respectivamente. Analisando a estrutura dividida em um número qualquer de subdomínios, denominados elementos finitos, aplicou-se a equação dos trabalhos interno e externo a cada um dos elementos. Adotando aproximações para os deslocamentos e deformações e minimizando a energia potencial total, chegou-se a um sistema de equações para cada elemento, definindo o conceito de matriz de rigidez e vetor de cargas nodais. A partir das matrizes de rigidez e vetores de cargas nodais dos elementos, foi possível montar uma única matriz e um único vetor válidos para toda a estrutura. Após considerar as condições de contorno do problema, resolve-se o sistema de equações, obtendom deslocamentos nos nós definidos na estrutura em questão.

As lajes do edifício, que em ALMEIDA (2003a) foram simuladas por diafragmas rígidos, foram modificadas neste trabalho. Estas são agora também modeladas pelo elemento DKT/FF. Os elementos reticulares também foram modificados, possuindo agora seis graus de liberdade por nó ao invés de cinco. 


\section{Capítulo 4}

\section{O Método da Rigidez Sucessiva}

\subsection{Introdução}

Neste capítulo analisa-se um solo formado por um número qualquer de camadas as quais podem conter um número qualquer de estacas, sendo que a camada mais profunda se encontra apoiada em uma superfície de deslocamento nulo. É obtida então uma matriz que representa a influência de todas estas camadas e de todas as estacas contidas nelas. Para cumprir este objetivo, é apresentada uma formulação baseada no Método da Rigidez Sucessiva (MRS) apresentada em MAIER \& NOVATI (1987). Estes procedimentos teóricos são base de parte do programa computacional descrito em ALMEIDA (2003a), utilizado neste trabalho.

Na formulação apresentada, o vetor de forças de superfície é relacionado ao vetor de deslocamentos por meio de uma matriz. Esta relação, por ser semelhante àquela proveniente do MEF, é o motivo pelo qual o termo "Método da Rigidez Sucessiva" (MRS) é empregado em MAIER \& NOVATI (1987). Apesar disto, a matriz obtida por este método não pode ser considerada de rigidez por não ser simétrica tampouco positiva definida. É dito "sucessiva" porque parte-se da camada em contato com a superfície de deslocamento nulo, incluindo então a influência de cada camada de baixo para cima. O processo é repetido iterativamente até que a superfície livre do solo seja atingida.

Iniciando as descrições desta teoria, na seção 4.2 o MRS é aplicado ao solo tridimensional sem estacas. São apresentadas as deduções para a obtenção da matriz de influência do solo e para o cálculo dos valores de contorno de todas as camadas.

Em seguida, é demonstrado na seção 4.3 como incluir a influência das estacas na formulação do MRS. O resultado é uma matriz referente a uma camada genérica cruzada por um número qualquer de estacas, computando toda a influência em função dos nós de topo e base da camada e das estacas. A partir desta matriz torna-se possível aplicar o MRS da mesma forma que na seção 4.2, obtendo todos 
os valores de contorno referentes às camadas e estacas.

\subsection{O MRS aplicado ao solo estratificado}

Para as deduções feitas nesta seção, são válidas as seguintes hipóteses:

- As camadas do solo são formadas por material elástico-linear, isotrópico e homogêneo.

- A camada mais profunda está apoiada em uma superfície de deslocamento nulo. Por este motivo, os deslocamentos nas três direções de todos os nós situados em sua base são prescritos iguais a zero.

- Em todos os nós do topo da camada superior, correspondente à superfície livre do solo, a força está prescrita nas três direções. Esta é a única superfície na qual o método admite cargas externas aplicadas.

- As camadas, representadas por domínios sólidos tridimensionais, são extensas em planta quando comparadas com a área de aplicação da carga. Esta hipótese permite supor que os deslocamentos estabelecidos no contorno lateral das camadas é desprezível.

Considera-se o caso geral de um solo formado por $N$ camadas, cada qual com uma espessura e diferentes características físicas, conforme ilustrado na figura 4.1. Nesta figura, $E_{j}$ é o módulo de elasticidade de uma camada $j$ qualquer e $\nu_{j}$ é seu coeficiente de Poisson. O domínio da camada está denominado por $\Omega_{j}$. Numera-se as camadas de baixo para cima.

Seja uma camada $i$ qualquer como apresentado na figura 4.2, sendo seu contorno tratado por $\Gamma$. Os subscritos $t, s$ e $b$ são correspondentes a, respectivamente, o topo, as laterais e a base da camada. Sendo ela um domínio tridimensional, é possível aplicar a formulação apresentada no capítulo 2 e obter a seguinte equação:

$$
\left[\mathbf{H}^{\mathrm{i}}\right]\left\{\mathbf{u}^{\mathrm{i}}\right\}=\left[\mathbf{G}^{\mathbf{i}}\right]\left\{\mathbf{p}^{\mathrm{i}}\right\}
$$

A equação 4.1 é válida somente para domínios elástico-lineares e isotrópicos. Os termos $\mathbf{H}^{\mathbf{i}}$ e $\mathbf{G}^{\mathbf{i}}$ são as matrizes de influência obtidas aplicando-se o MEC à camada $i$. O vetor $\mathbf{u}^{\mathbf{i}}$ contém os deslocamentos nas três direções de cada nó do contorno, sendo composto pelos subvetores $\mathbf{u}_{\mathbf{t}}, \mathbf{u}_{\mathbf{s}}$ e $\mathbf{u}_{\mathbf{b}}$ indicados na figura 4.1. O vetor $\mathbf{p}^{\mathbf{i}}$ contém as forças de superfície e é composto pelos subvetores $\mathbf{p}_{\mathbf{t}}, \mathbf{p}_{\mathbf{s}}$ e $\mathbf{p}_{\mathbf{b}}$. 


\begin{tabular}{|c|c|}
\hline \multicolumn{2}{|c|}{ Superficie livre do solo } \\
\hline $\mathrm{E}_{\mathrm{N}}, \nu_{\mathrm{N}}$ & $\Omega_{\mathrm{N}}$ \\
\hline & $\vdots$ \\
\hline $\mathrm{E}_{3}, \nu_{3}$ & $\Omega_{3}$ \\
\hline $\mathrm{E}_{2}, \nu_{2}$ & $\Omega_{2}$ \\
\hline $\mathrm{E}_{1}, \nu_{1}$ & $\Omega_{1}$ \\
\hline
\end{tabular}

Figura 4.1: Solo estratificado em camadas

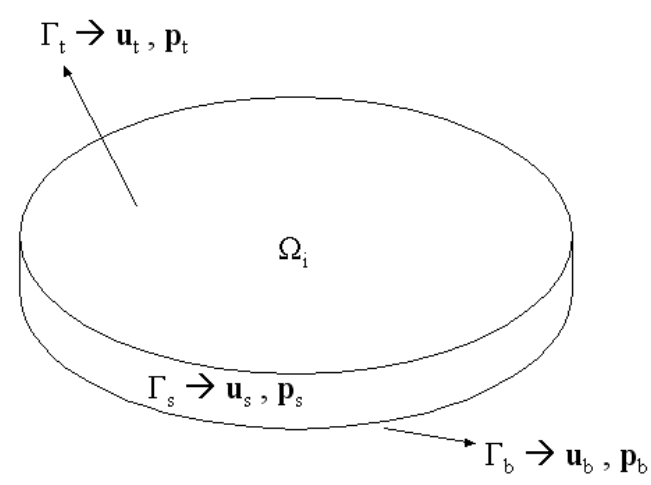

Figura 4.2: Camada $i$ isolada

Como a matriz $\mathbf{G}^{\mathbf{i}}$ é inversível, pode-se multiplicar ambos os lados da equação 4.1 por sua inversa obtendo:

$$
\left[\mathbf{G}^{\mathrm{i}}\right]^{-1}\left[\mathbf{H}^{\mathbf{i}}\right]\left\{\mathbf{u}^{\mathrm{i}}\right\}=\left\{\mathbf{p}^{\mathrm{i}}\right\}
$$

Efetuando o produto matricial à esquerda de 4.2 chega-se à equação:

$$
\left[\mathbf{K}^{\mathbf{i}}\right]\left\{\mathbf{u}^{\mathrm{i}}\right\}=\left\{\mathbf{p}^{\mathrm{i}}\right\}
$$

com

$$
\left[\mathbf{K}^{\mathrm{i}}\right]=\left[\mathbf{G}^{\mathrm{i}}\right]^{-\mathbf{1}}\left[\mathbf{H}^{\mathrm{i}}\right]
$$

A equação 4.3, decomposta nas parcelas do contorno referentes ao topo, às laterais e à base da camada $i$, se torna:

$$
\left[\begin{array}{lll}
K_{t t}^{i} & K_{t b}^{i} & K_{t s}^{i} \\
K_{b t}^{i} & K_{b b}^{i} & K_{b s}^{i} \\
K_{s t}^{i} & K_{s b}^{i} & K_{s s}^{i}
\end{array}\right]\left\{\begin{array}{c}
u_{t}^{i} \\
u_{b}^{i} \\
u_{s}^{i}
\end{array}\right\}=\left\{\begin{array}{l}
p_{t}^{i} \\
p_{b}^{i} \\
p_{s}^{i}
\end{array}\right\}
$$

Nas submatrizes de $\mathbf{K}^{\mathbf{i}}$, o primeiro índice subscrito indica a parcela do contorno que gerou a linha. Já o segundo faz referência ao subvetor de $\mathbf{u}^{\mathbf{i}}$ que multiplica a 
submatriz. O índice sobrescrito $i$ indica o número da camada para a qual se está escrevendo a equação.

Por 4.5, pode-se obter uma equação para cada camada do solo. Estas equações podem ser simplificadas considerando-se a quarta hipótese apresentada no início desta seção. Deste modo, pode-se igualar $\mathbf{u}_{\mathbf{s}}^{\mathrm{i}}$ a zero e reescrever 4.5 da seguinte forma:

$$
\left[\begin{array}{lll}
K_{t t}^{i} & K_{t b}^{i} & K_{t s}^{i} \\
K_{b t}^{i} & K_{b b}^{i} & K_{b s}^{i} \\
K_{s t}^{i} & K_{s b}^{i} & K_{s s}^{i}
\end{array}\right]\left\{\begin{array}{c}
u_{t}^{i} \\
u_{b}^{i} \\
0
\end{array}\right\}=\left\{\begin{array}{c}
p_{t}^{i} \\
p_{b}^{i} \\
p_{s}^{i}
\end{array}\right\}
$$

Considerando somente as duas primeiras linhas de 4.6, nota-se que a terceira coluna da matriz desaparece, restando:

$$
\left[\begin{array}{ll}
K_{t t}^{i} & K_{t b}^{i} \\
K_{b t}^{i} & K_{b b}^{i}
\end{array}\right]\left\{\begin{array}{l}
u_{t}^{i} \\
u_{b}^{i}
\end{array}\right\}=\left\{\begin{array}{l}
p_{t}^{i} \\
p_{b}^{i}
\end{array}\right\}
$$

A terceira linha de 4.6 poderá ser utilizada posteriormente para calcular as forças nos nós do contorno lateral das camadas. Para isto, depois de determinar os deslocamentos para a camada $i$, aplica-se a relação:

$$
\left\{\mathbf{p}_{\mathrm{s}}^{\mathrm{i}}\right\}=\left[\mathbf{K}_{\mathrm{st}}^{\mathrm{i}}\right]\left\{\mathbf{u}_{\mathrm{t}}^{\mathrm{i}}\right\}+\left[\mathbf{K}_{\mathrm{sb}}^{\mathrm{i}}\right]\left\{\mathbf{u}_{\mathrm{b}}^{\mathrm{i}}\right\}
$$

É agora que se inicia o processo iterativo do MRS para a obtenção da matriz final de influência do solo. Considera-se a equação 4.7 para a camada que está em contato com a superfície de deslocamento nulo, ou seja, $i$ igual a 1:

$$
\left[\begin{array}{ll}
K_{\mathrm{tt}}^{1} & \mathbf{K}_{\mathrm{tb}}^{1} \\
\mathbf{K}_{\mathrm{bt}}^{1} & \mathbf{K}_{\mathrm{bb}}^{1}
\end{array}\right]\left\{\begin{array}{l}
\mathbf{u}_{\mathrm{t}}^{1} \\
\mathbf{u}_{\mathrm{b}}^{1}
\end{array}\right\}=\left\{\begin{array}{l}
\mathbf{p}_{\mathrm{t}}^{1} \\
\mathbf{p}_{\mathrm{b}}^{1}
\end{array}\right\}
$$

A segunda hipótese assumida no início desta seção garante que os deslocamentos na base da camada 1 são iguais a zero. Isto permite reescrever a equação 4.9 como:

$$
\left[\begin{array}{ll}
K_{t t}^{1} & K_{t b}^{1} \\
K_{b t}^{1} & K_{b b}^{1}
\end{array}\right]\left\{\begin{array}{c}
u_{t}^{1} \\
0
\end{array}\right\}=\left\{\begin{array}{l}
p_{t}^{1} \\
p_{b}^{1}
\end{array}\right\}
$$

A primeira linha de 4.10 isolada fornece:

$$
\left[\mathbf{K}_{\mathbf{t t}}^{\mathbf{1}}\right]\left\{\mathbf{u}_{\mathbf{t}}^{1}\right\}=\left\{\mathbf{p}_{\mathbf{t}}^{1}\right\}
$$

$\mathrm{Ou}$

$$
\left[\hat{\mathbf{K}}^{1}\right]\left\{\mathbf{u}_{\mathbf{t}}^{1}\right\}=\left\{\mathbf{p}_{\mathbf{t}}^{1}\right\}
$$


com

$$
\left[\hat{\mathbf{K}}^{1}\right]=\left[\mathbf{K}_{\mathbf{t t}}^{\mathbf{1}}\right]
$$

A equação 4.12 relaciona os deslocamentos do topo da camada $1 \mathrm{com}$ as forças de superfície atuantes neste contorno por meio da matriz $\hat{\mathbf{K}}^{\mathbf{1}}$. Nesta matriz estão condensadas as influências de todos os nós do contorno da camada, incluindo os da lateral e da base.

Para relacionar a camada 1 com a camada 2, aplica-se na superfície de contato entre elas condições de equilíbrio de forças e compatibilidade de deslocamentos, conforme ilustrado na figura 4.3 .

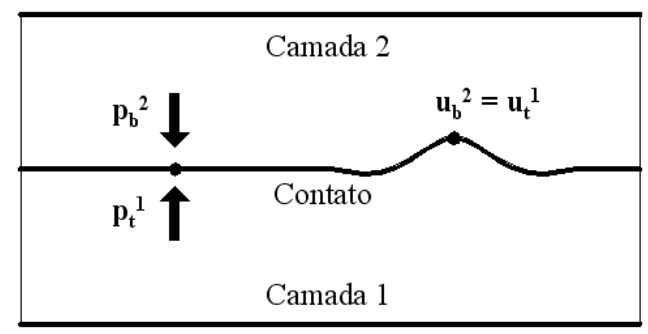

Figura 4.3: Equilíbrio e compatibilidade entre as camadas 1 e 2

Para representar estas condições, escreve-se as igualdades:

$$
\begin{gathered}
\left\{\mathbf{p}_{\mathrm{t}}^{1}\right\}=-\left\{\mathbf{p}_{\mathrm{b}}^{2}\right\} \\
\left\{\mathbf{u}_{\mathrm{t}}^{1}\right\}=\left\{\mathbf{u}_{\mathrm{b}}^{2}\right\}
\end{gathered}
$$

O passo seguinte é obter a equação 4.9 para a camada 2, ou seja:

$$
\left[\begin{array}{ll}
K_{\mathrm{tt}}^{2} & \mathrm{~K}_{\mathrm{tb}}^{2} \\
\mathrm{~K}_{\mathrm{bt}}^{2} & \mathrm{~K}_{\mathrm{bb}}^{2}
\end{array}\right]\left\{\begin{array}{l}
\mathbf{u}_{\mathrm{t}}^{2} \\
\mathbf{u}_{\mathrm{b}}^{2}
\end{array}\right\}=\left\{\begin{array}{l}
\mathrm{p}_{\mathrm{t}}^{2} \\
\mathrm{p}_{\mathrm{b}}^{2}
\end{array}\right\}
$$

Aplicando as igualdades 4.14, 4.15 e 4.12 em 4.16, é possível obter uma relação semelhante a 4.12 mas para a camada 2. Em princípio, escreve-se a segunda linha de 4.16 de forma isolada:

$$
\left[\mathbf{K}_{\mathbf{b t}}^{2}\right]\left\{\mathbf{u}_{\mathbf{t}}^{2}\right\}+\left[\mathbf{K}_{\mathbf{b b}}^{2}\right]\left\{\mathbf{u}_{\mathrm{b}}^{2}\right\}=\left\{\mathbf{p}_{\mathbf{b}}^{2}\right\}
$$

Aplicando as igualdades 4.14 e 4.15 em 4.17, obtém-se:

$$
\left[\mathbf{K}_{\mathrm{bt}}^{2}\right]\left\{\mathbf{u}_{\mathrm{t}}^{2}\right\}+\left[\mathbf{K}_{\mathrm{bb}}^{2}\right]\left\{\mathbf{u}_{\mathbf{t}}^{1}\right\}=-\left\{\mathbf{p}_{\mathrm{t}}^{1}\right\}
$$

Agora é possível substituir a expressão 4.12 ao lado direito de 4.18 , chegando à relação:

$$
\left[\mathbf{K}_{\mathbf{b t}}^{2}\right]\left\{\mathbf{u}_{\mathbf{t}}^{2}\right\}+\left[\mathbf{K}_{\mathbf{b b}}^{2}\right]\left\{\mathbf{u}_{\mathbf{t}}^{1}\right\}=-\left[\hat{\mathbf{K}}^{1}\right]\left\{\mathbf{u}_{\mathbf{t}}^{\mathbf{1}}\right\}
$$


Na sequência, isola-se o vetor $\mathbf{u}_{\mathbf{t}}^{\mathbf{1}}$ em 4.19 , ou seja:

$$
\begin{gathered}
{\left[\mathbf{K}_{\mathrm{bt}}^{2}\right]\left\{\mathbf{u}_{\mathbf{t}}^{2}\right\}=\left(-\left[\mathbf{K}_{\mathbf{b b}}^{2}\right]-\left[\hat{\mathbf{K}}^{\mathbf{1}}\right]\right)\left\{\mathbf{u}_{\mathbf{t}}^{\mathbf{1}}\right\}} \\
\left\{\mathbf{u}_{\mathbf{t}}^{\mathbf{1}}\right\}=\left(-\left[\mathbf{K}_{\mathrm{bb}}^{2}\right]-\left[\hat{\mathbf{K}}^{\mathbf{1}}\right]\right)^{-\mathbf{1}}\left[\mathbf{K}_{\mathbf{b t}}^{2}\right]\left\{\mathbf{u}_{\mathbf{t}}^{2}\right\}
\end{gathered}
$$

Esta expressão obtida para $\mathbf{u}_{\mathbf{t}}^{\mathbf{1}}$ deve ser guardada para ser utilizada mais adiante. Tomando agora a primeira linha de 4.16, escreve-se:

$$
\left[\mathbf{K}_{\mathrm{tt}}^{2}\right]\left\{\mathbf{u}_{\mathrm{t}}^{2}\right\}+\left[\mathbf{K}_{\mathrm{tb}}^{2}\right]\left\{\mathbf{u}_{\mathrm{b}}^{2}\right\}=\left\{\mathbf{p}_{\mathrm{t}}^{2}\right\}
$$

Aplicando a relação 4.15, pode-se reescrever 4.22 como:

$$
\left[\mathbf{K}_{\mathrm{tt}}^{2}\right]\left\{\mathbf{u}_{\mathrm{t}}^{2}\right\}+\left[\mathbf{K}_{\mathrm{tb}}^{2}\right]\left\{\mathbf{u}_{\mathrm{t}}^{1}\right\}=\left\{\mathbf{p}_{\mathrm{t}}^{2}\right\}
$$

Para continuar as deduções, deve-se agora substituir a expressão 4.21 obtida para $\mathbf{u}_{\mathbf{t}}^{\mathbf{1}}$ na igualdade 4.23 , obtendo a equação:

$$
\left[\mathbf{K}_{\mathrm{tt}}^{2}\right]\left\{\mathbf{u}_{\mathbf{t}}^{2}\right\}+\left[\mathbf{K}_{\mathrm{tb}}^{2}\right]\left(\left(-\left[\mathbf{K}_{\mathbf{b b}}^{2}\right]-\left[\hat{\mathbf{K}}^{1}\right]\right)^{-1}\left[\mathbf{K}_{\mathbf{b t}}^{2}\right]\left\{\mathbf{u}_{\mathbf{t}}^{2}\right\}\right)=\left\{\mathbf{p}_{\mathbf{t}}^{2}\right\}
$$

Para concluir a dedução para a camada 2, deve-se trabalhar a equação 4.24 para obter uma relação mais compacta. Tem-se então o equacionamento:

$$
\left(\left[\mathbf{K}_{\mathbf{t t}}^{2}\right]+\left[\mathbf{K}_{\mathbf{t b}}^{2}\right]\left(\left(-\left[\mathbf{K}_{\mathbf{b b}}^{2}\right]-\left[\hat{\mathbf{K}}^{1}\right]\right)^{-1}\left[\mathbf{K}_{\mathbf{b t}}^{2}\right]\right)\right)\left\{\mathbf{u}_{\mathbf{t}}^{2}\right\}=\left\{\mathbf{p}_{\mathbf{t}}^{2}\right\}
$$

Desenvolvendo a equação 4.25 , todos os termos que multiplicam $\mathbf{u}_{\mathbf{t}}^{2}$ se reduzem a uma única matriz. Assim, a equação 4.25 se torna:

$$
\left[\hat{\mathbf{K}}^{2}\right]\left\{\mathbf{u}_{\mathbf{t}}^{2}\right\}=\left\{\mathbf{p}_{\mathbf{t}}^{2}\right\}
$$

A equação 4.25 relaciona os deslocamentos nos nós do topo da camada 2 com as forças nestes nós por meio da matriz $\hat{\mathbf{K}}^{\mathbf{2}}$. Nesta matriz estão presentes as influências de todo o contorno da camada 2 e também de todo o contorno da camada 1 . Conforme pode ser observado, o procedimento adotado entre a equação 4.12 e a equação 4.26 é válido para quaisquer camadas subsequentes $i$ e $i+1$. Portanto, isto poderia ser repetido mais uma vez obtendo, para a camada 3 , a equação:

$$
\left[\hat{\mathbf{K}}^{3}\right]\left\{\mathbf{u}_{\mathbf{t}}^{3}\right\}=\left\{\mathbf{p}_{\mathbf{t}}^{3}\right\}
$$

Da mesma forma, poderiam ser obtidas as equações:

$$
\left[\hat{\mathbf{K}}^{4}\right]\left\{\mathbf{u}_{\mathbf{t}}^{4}\right\}=\left\{\mathbf{p}_{\mathbf{t}}^{4}\right\}
$$




$$
\left[\hat{\mathbf{K}}^{5}\right]\left\{\mathbf{u}_{\mathbf{t}}^{5}\right\}=\left\{\mathbf{p}_{\mathbf{t}}^{\mathbf{5}}\right\}
$$

e assim sucessivamente.

O MRS repete este processo iterativo até que seja atingida a camada da superfície livre do solo. A ordem de obtenção das matrizes se dá, portanto, da camada 1 para a camada $N$. Isto está ilustrado na figura 4.4 .

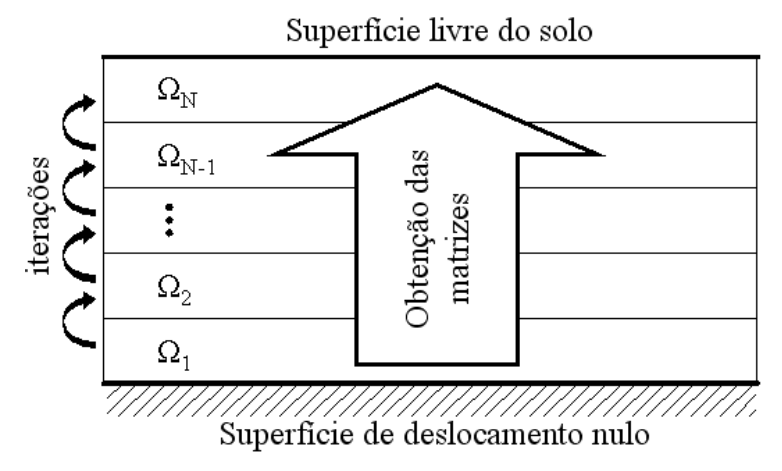

Figura 4.4: Ordem de obtenção das matrizes no processo iterativo

Para a camada $N$ obtém-se:

$$
\left[\hat{\mathbf{K}}^{\mathbf{N}}\right]\left\{\mathbf{u}_{\mathbf{t}}^{\mathbf{N}}\right\}=\left\{\mathbf{p}_{\mathbf{t}}^{\mathbf{N}}\right\}
$$

$\mathrm{O}$ vetor $\mathbf{u}_{\mathbf{t}}^{\mathbf{N}}$ contém os deslocamentos nas três direções nos nós da superfície livre do solo, e o vetor $\mathbf{p}_{\mathbf{t}}^{\mathbf{N}}$ contém as cargas externas atuantes nesta superfície. O vetor $\mathbf{p}_{\mathbf{t}}^{\mathbf{N}}$ é conhecido, conforme a terceira hipótese feita ao início desta seção. A matriz $\hat{\mathbf{K}}^{\mathbf{N}}$ contém a influência de todas as camadas do solo, e relaciona os deslocamentos dos nós da superfície livre do solo com as forças de superfície nestes nós.

Sendo conhecidos todos os termos da matriz $\hat{\mathbf{K}}^{\mathbf{N}}$ e do vetor $\mathbf{p}_{\mathbf{t}}^{\mathbf{N}}$, pode-se determinar os termos do vetor $\mathbf{u}_{\mathbf{t}}^{\mathbf{N}}$ resolvendo-se o sistema de equações lineares 4.30 . Determinados os deslocamentos na superfície livre do solo, fica encerrada a primeira parte do MRS.

Na segunda etapa são determinados todos os valores de contorno que permaneceram incógnitos em todas as camadas, começando pela camada $N$. A equação 4.7. escrita para a camada $N$, se torna:

$$
\left[\begin{array}{ll}
\mathbf{K}_{\mathrm{tt}}^{\mathrm{N}} & \mathbf{K}_{\mathrm{tb}}^{\mathrm{N}} \\
\mathbf{K}_{\mathrm{bt}}^{\mathrm{N}} & \mathbf{K}_{\mathrm{bb}}^{\mathrm{N}}
\end{array}\right]\left\{\begin{array}{l}
\mathbf{u}_{\mathrm{t}}^{\mathrm{N}} \\
\mathbf{u}_{\mathrm{b}}^{\mathbf{N}}
\end{array}\right\}=\left\{\begin{array}{l}
\mathbf{p}_{\mathrm{t}}^{\mathrm{N}} \\
\mathbf{p}_{\mathrm{b}}^{\mathbf{N}}
\end{array}\right\}
$$

Neste ponto são conhecidas todas as submatrizes à esquerda de 4.31 e os subvetores $\mathbf{u}_{\mathbf{t}}^{\mathbf{N}}$ e $\mathbf{p}_{\mathbf{t}}^{\mathbf{N}}$. A primeira linha de 4.31 escrita isoladamente é:

$$
\left[\mathbf{K}_{\mathbf{t t}}^{\mathbf{N}}\right]\left\{\mathbf{u}_{\mathbf{t}}^{\mathbf{N}}\right\}+\left[\mathbf{K}_{\mathbf{t b}}^{\mathbf{N}}\right]\left\{\mathbf{u}_{\mathbf{b}}^{\mathbf{N}}\right\}=\left\{\mathbf{p}_{\mathbf{t}}^{\mathbf{N}}\right\}
$$


Como se pode observar o único termo desconhecido de 4.32 é o vetor $\mathbf{u}_{\mathbf{b}}^{\mathbf{N}}$, que contém os deslocamentos nas três direções dos nós da base da camada $N$. Para determiná-lo basta isolá-lo em 4.32, ou seja:

$$
\begin{gathered}
{\left[\mathbf{K}_{\mathrm{tb}}^{\mathbf{N}}\right]\left\{\mathbf{u}_{\mathbf{b}}^{\mathbf{N}}\right\}=\left\{\mathbf{p}_{\mathbf{t}}^{\mathbf{N}}\right\}-\left[\mathbf{K}_{\mathbf{t t}}^{\mathbf{N}}\right]\left\{\mathbf{u}_{\mathbf{t}}^{\mathbf{N}}\right\}} \\
\left\{\mathbf{u}_{\mathbf{b}}^{\mathbf{N}}\right\}=\left[\mathbf{K}_{\mathbf{t b}}^{\mathbf{N}}\right]^{-1}\left\{\mathbf{p}_{\mathbf{t}}^{\mathbf{N}}\right\}-\left[\mathbf{K}_{\mathbf{t b}}^{\mathbf{N}}\right]^{-1}\left[\mathbf{K}_{\mathrm{tt}}^{\mathbf{N}}\right]\left\{\mathbf{u}_{\mathbf{t}}^{\mathbf{N}}\right\}
\end{gathered}
$$

Após determinar os deslocamentos dos nós da base, pode-se determinar as forças nos nós da base. A segunda linha de 4.31é:

$$
\left[\mathbf{K}_{\mathbf{b t}}^{\mathbf{N}}\right]\left\{\mathbf{u}_{\mathbf{t}}^{\mathbf{N}}\right\}+\left[\mathbf{K}_{\mathbf{b b}}^{\mathbf{N}}\right]\left\{\mathbf{u}_{\mathbf{b}}^{\mathbf{N}}\right\}=\left\{\mathbf{p}_{\mathbf{b}}^{\mathbf{N}}\right\}
$$

O único termo desconhecido na relação 4.35 é o subvetor $\mathbf{p}_{\mathbf{b}}^{\mathbf{N}}$, que pode ser determinado diretamente desta igualdade. Os deslocamentos nos nós do contorno lateral da camada $N$ são nulos, segundo a quarta hipótese feita no início da seção. Os únicos valores de contorno que continuam desconhecidos para a camada $N$ são as forças nos nós do contorno lateral. Estas podem ser determinadas aplicando-se a relação 4.8 à camada $N$, ou seja:

$$
\left\{\mathbf{p}_{\mathbf{s}}^{\mathbf{N}}\right\}=\left[\mathbf{K}_{\mathbf{s t}}^{\mathbf{N}}\right]\left\{\mathbf{u}_{\mathbf{t}}^{\mathbf{N}}\right\}+\left[\mathbf{K}_{\mathbf{s b}}^{\mathbf{N}}\right]\left\{\mathbf{u}_{\mathbf{b}}^{\mathbf{N}}\right\}
$$

Assim, ficam conhecidos todos os valores de contorno relativos à camada $N$. Determinados os valores de contorno, torna-se possível calcular deslocamentos e tensões em nós internos à camada $N$ aplicando as expressões apresentadas no capítulo anterior.

Como os deslocamentos e forças na base da camada $N$ são conhecidos, pode-se obter os deslocamentos e forças no topo da camada $N-1$. Para isto basta aplicar as condições de equilíbrio 4.14 e compatibilidade 4.15 na interface das camadas $N$ e $N-1$, ou seja:

$$
\begin{gathered}
\left\{\mathbf{p}_{\mathbf{t}}^{\mathbf{N}-\mathbf{1}}\right\}=-\left\{\mathbf{p}_{\mathbf{b}}^{\mathbf{N}}\right\} \\
\left\{\mathbf{u}_{\mathbf{t}}^{\mathbf{N}-\mathbf{1}}\right\}=\left\{\mathbf{u}_{\mathbf{b}}^{\mathbf{N}}\right\}
\end{gathered}
$$

Concluindo as deduções escreve-se a equação 4.7 para a camada $N-1$, isto é:

$$
\left[\begin{array}{ll}
\mathrm{K}_{\mathrm{tt}}^{\mathrm{N}-1} & \mathrm{~K}_{\mathrm{tb}}^{\mathrm{N}-1} \\
\mathrm{~K}_{\mathrm{bt}}^{\mathrm{N}-1} & \mathrm{~K}_{\mathrm{bb}}^{\mathrm{N}-1}
\end{array}\right]\left\{\begin{array}{l}
\mathbf{u}_{\mathrm{t}}^{\mathrm{N}-1} \\
\mathbf{u}_{\mathrm{b}}^{\mathrm{N}-1}
\end{array}\right\}=\left\{\begin{array}{l}
\mathrm{p}_{\mathrm{t}}^{\mathrm{N}-1} \\
\mathrm{p}_{\mathrm{b}}^{\mathrm{N}-1}
\end{array}\right\}
$$

Para determinar todos os valores de contorno da camada $N-1$ e chegar ao ponto de partida da camada $N-2$, basta repetir o equacionamento feito entre as igualdades 4.31 e 4.39. Conhecidos os valores de contorno da camada $N-1$, 
torna-se possível calcular deslocamentos e tensões em pontos internos a esta camada também. O procedimento entre as equações 4.31 e 4.39 é repetido até a camada número 1, quando ficam conhecidos os valores de contorno de todas as camadas. Isto possibilita o cálculo de deslocamentos e tensões em pontos internos a qualquer camada. A ordem do cálculo dos valores de contorno no processo iterativo se dá, portanto, da camada $N$ para a camada 1 . Isto está ilustrado na figura 4.5 .

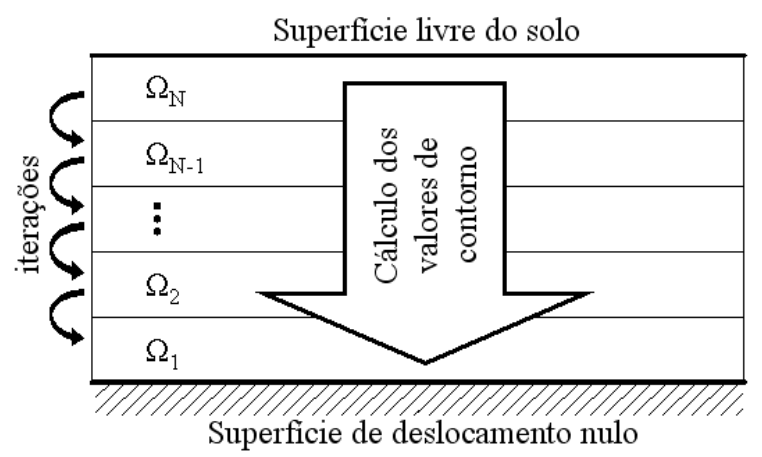

Figura 4.5: Ordem do cálculo dos valores de contorno no processo iterativo

\subsection{O MRS aplicado ao solo com estacas}

Na seção 4.2, foi apresentado o MRS para camadas de solo sem estacas. O ponto de partida do método foi a equação 4.7, na qual a influência de todo o contorno de uma camada $i$ qualquer ficou representada apenas pelo nós de topo e base da camada $i$. Nesta seção é obtida uma equação similar à equação 4.7, porém também considerando a influência de um número qualquer de estacas cruzando a camada $i$. Se torna possível então aplicar o MRS de forma muito semelhante à apresentada na seção 4.2, obtendo todos os valores de contorno referentes a todas as camadas e estacas.

As hipóteses iniciais a serem consideradas nesta seção, além das da seção anterior, são as seguintes:

- Quando uma estaca cruza mais de uma camada, ela fica dividida em segmentos. O comprimento de um segmento é sempre igual à expessura da camada à qual este pertence, e o equilíbrio entre camadas adjacentes garante a continuidade de todas as estacas.

- Cada camada, assim como os segmentos de estaca, é um sólido tridimensional no qual o MEC pode ser aplicado.

Considerando a segunda hipótese adotada, inicia-se as deduções escrevendo, para 
uma camada $i$ qualquer, a equação:

$$
\left[\mathbf{H}^{\mathrm{i}}\right]\left\{\mathbf{u}^{\mathrm{i}}\right\}=\left[\mathbf{G}^{\mathrm{i}}\right]\left\{\mathbf{p}^{\mathrm{i}}\right\}
$$

que é igual à equação 4.1 escrita para uma camada $i$ qualquer na seção 4.2. Também como foi feito na seção anterior pode-se obter, a partir da equação 4.40, a equação:

$$
\left[\mathbf{K}^{\mathbf{i}}\right]\left\{\mathbf{u}^{\mathbf{i}}\right\}=\left\{\mathbf{p}^{\mathbf{i}}\right\}
$$

com

$$
\left[\mathbf{K}^{\mathbf{i}}\right]=\left[\mathbf{G}^{\mathbf{i}}\right]^{-\mathbf{1}}\left[\mathbf{H}^{\mathbf{i}}\right]
$$

A diferença é que agora pretende-se considerar um número qualquer de segmentos de estaca atravessando totalmente a camada $i$. O contorno da camada fica modificado pela inclusão dos trechos correspondentes aos contatos entre o solo e as estacas, além da exclusão dos trechos correspondentes ao topo e base de cada estaca. Tratando o topo da camada pelo índice subscrito $t$, a base pelo índice subscrito $b$ e o contato com o fuste de uma estaca qualquer pelo índice subscrito $f$, pode-se visualizar na figura 4.6 a camada $i$ cruzada por um único segmento de estaca.

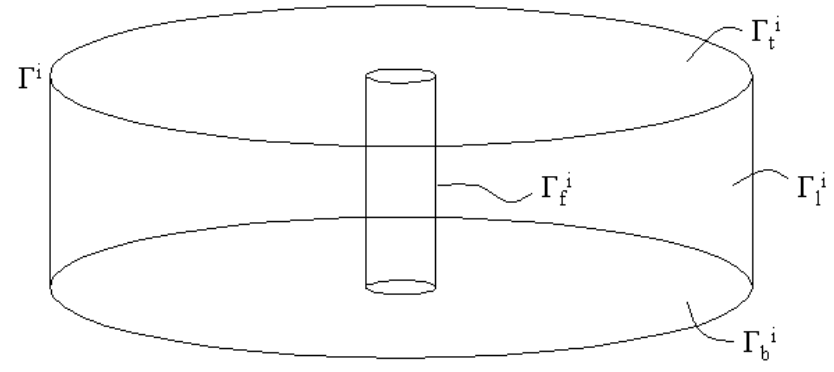

Figura 4.6: Contorno da camada $i$ dividido em trechos

Como se pode observar na figura 4.6, a camada $i$ é um sólido com um furo. O índice sobrescrito $i$ indica o número da camada. O contorno da camada, indicado por $\Gamma^{i}$, está dividido em quatro trechos. O trecho $\Gamma_{t}^{i}$ corresponde ao topo da camada, $\Gamma_{b}^{i}$ corresponde à base da camada, $\Gamma_{l}^{i}$ às laterais e $\Gamma_{f}^{i}$ ao contato entre solo e estaca. Cada uma destas parcelas do contorno deve ser discriminada na equação 4.41 para tornar possível as deduções. Para tratar de um caso mais geral, considera-se uma camada $i$ cruzada por um número qualquer de estacas. Este caso é apresentado esquematicamente na figura 4.7.

Na figura 4.7, aparecem dois índices sobrescritos na indicação das parcelas do contorno $\Gamma^{i}$ correspondentes às estacas. O primeiro indica o número da camada e o segundo o número da estaca. O número total de estacas encontra-se indicado pela letra $n$. 


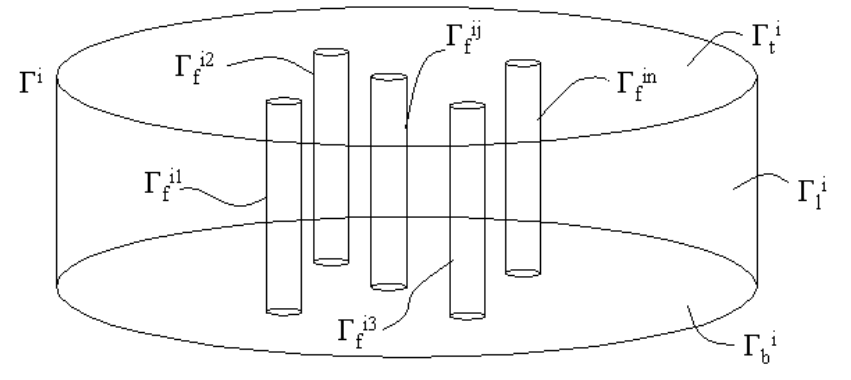

Figura 4.7: Caso mais geral para uma camada $i$

Para o sólido tridimensional ilustrado na figura 4.7, a equação 4.41 pode ser escrita discriminando cada parcela do contorno. Esta equação então se torna:

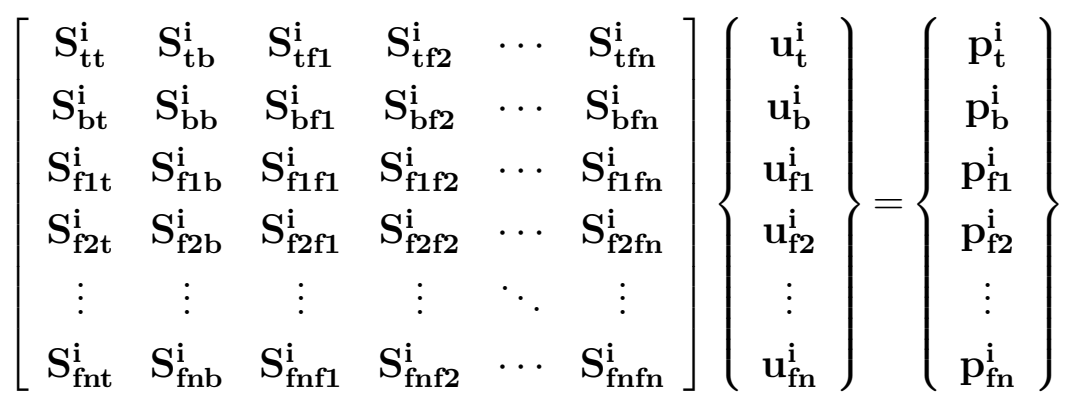

Foi eliminada da equação 4.43 a influência das laterais da camada, igualando os deslocamentos das laterais a zero. Este mesmo procedimento foi feito na seção anterior, entre as equações 4.5 e 4.7.

Após obter a equação 4.43 , deve-se deduzir uma expressão semelhante para cada estaca imersa na camada $i$. Considera-se uma estaca genérica $j$, conforme ilustrado na figura 4.8 .

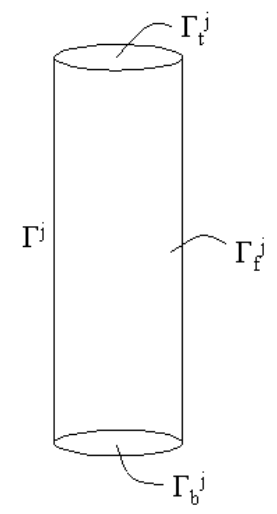

Figura 4.8: Estaca $j$ genérica

Considerando cada trecho do contorno, conclui-se que a equação 4.41 escrita para 
uma estaca genérica $j$ é:

$$
\left[\begin{array}{ccc}
E_{t t}^{j} & E_{t b}^{j} & E_{t f}^{j} \\
E_{b t}^{j} & E_{b b}^{j} & E_{b f}^{j} \\
E_{f t}^{j} & E_{b t}^{j} & E_{f f}^{j}
\end{array}\right]\left\{\begin{array}{c}
u_{t}^{j} \\
u_{b}^{j} \\
u_{f}^{j}
\end{array}\right\}=\left\{\begin{array}{c}
p_{t}^{j} \\
p_{b}^{j} \\
p_{f}^{j}
\end{array}\right\}
$$

Para relacionar as equações 4.43 e 4.44 , pode-se utilizar condições de equilíbrio de forças e compatibilidade de deslocamentos que existem no contato entre a estaca $j$ e a camada $i$. Estas condições são traduzidas pelas relações:

$$
\left\{\mathbf{u}_{\mathrm{fj}}^{\mathrm{i}}\right\}=\left\{\mathbf{u}_{\mathrm{f}}^{\mathrm{j}}\right\}
$$

e

$$
\left\{\mathbf{p}_{\mathrm{fj}}^{\mathrm{i}}\right\}=-\left\{\mathbf{p}_{\mathbf{f}}^{\mathbf{j}}\right\}
$$

A condição de compatibilidade de deslocamentos 4.45 pode ser aplicada diretamente à equação 4.43 . Após as substituições, esta se torna:

$$
\left[\begin{array}{cccccc}
S_{t t}^{i} & S_{t b}^{i} & S_{t f 1}^{i} & S_{t f 2}^{i} & \cdots & S_{t f n}^{i} \\
S_{b t}^{i} & S_{b b}^{i} & S_{b f 1}^{i} & S_{b f 2}^{i} & \cdots & S_{b f n}^{i} \\
S_{f 1 t}^{i} & S_{f 1 b}^{i} & S_{f 1 f 1}^{i} & S_{f 1 f 2}^{i} & \cdots & S_{f 1 f n}^{i} \\
S_{f 2 t}^{i} & S_{f 2 b}^{i} & S_{f 2 f 1}^{i} & S_{f 2 f 2}^{i} & \cdots & S_{f 2 f n}^{i} \\
\vdots & \vdots & \vdots & \vdots & \ddots & \vdots \\
S_{f n t}^{i} & S_{f n b}^{i} & S_{f n f 1}^{i} & S_{f n f 2}^{i} & \cdots & S_{f n f n}^{i}
\end{array}\right]\left\{\begin{array}{c}
u_{t}^{i} \\
u_{b}^{i} \\
u_{f}^{i} \\
u_{f}^{2} \\
\vdots \\
u_{f}^{n}
\end{array}\right\}=\left\{\begin{array}{c}
p_{t}^{i} \\
p_{b}^{i} \\
p_{f 1}^{i} \\
p_{f 2}^{i} \\
\vdots \\
p_{f n}^{i}
\end{array}\right\}
$$

A partir da equação 4.46 aplicada às equações 4.44 e 4.47, deve-se isolar os deslocamentos relacionados aos fustes de todas as estacas. Com isto será possível eliminá-los da equação, juntamente com as forças nos contornos referentes aos contatos. Inicialmente, isola-se da equação 4.47 as linhas correspondentes às forças nos fustes das estacas. Isto resulta na equação:

$$
\left[\begin{array}{cccccc}
S_{f 1 t}^{i} & S_{f 1 b}^{i} & S_{f 1 f 1}^{i} & S_{f 1 f 2}^{i} & \cdots & S_{f 1 f n}^{i} \\
S_{f 2 t}^{i} & S_{f 2 b}^{i} & S_{f 2 f 1}^{i} & S_{f 2 f 2}^{i} & \cdots & S_{f 2 f n}^{i} \\
\vdots & \vdots & \vdots & \vdots & \ddots & \vdots \\
S_{f n t}^{i} & S_{f n b}^{i} & S_{f n f 1}^{i} & S_{f n f 2}^{i} & \cdots & S_{f n f n}^{i}
\end{array}\right]\left\{\begin{array}{c}
u_{t}^{i} \\
u_{b}^{i} \\
u_{f}^{1} \\
u_{f}^{2} \\
\vdots \\
u_{f}^{n}
\end{array}\right\}=\left\{\begin{array}{c}
p_{f 1}^{i} \\
p_{f 2}^{i} \\
\vdots \\
p_{f n}^{i}
\end{array}\right\}
$$

Para seguir com as deduções, deve-se escrever a terceira linha da equação 4.44 para $j$ de 1 até $n$. É mais interessante que estas equações sejam organizadas matri- 
cialmente, resultando então em uma única equação. Esta equação é:

$$
\left[\begin{array}{ccccccccccc}
\mathbf{E}_{\mathrm{ft}}^{1} & \mathbf{E}_{\mathrm{fb}}^{1} & \mathbf{0} & \mathbf{0} & \cdots & \mathbf{0} & \mathbf{0} & \mathrm{E}_{\mathrm{ff}}^{1} & \mathbf{0} & \cdots & \mathbf{0} \\
\mathbf{0} & \mathbf{0} & \mathrm{E}_{\mathrm{ft}}^{2} & \mathrm{E}_{\mathrm{fb}}^{2} & \cdots & \mathbf{0} & \mathbf{0} & \mathbf{0} & \mathrm{E}_{\mathrm{ff}}^{2} & \cdots & \mathbf{0} \\
\vdots & \vdots & \vdots & \vdots & \ddots & \vdots & \vdots & \vdots & \vdots & \ddots & \vdots \\
\mathbf{0} & \mathbf{0} & \mathbf{0} & \mathbf{0} & \cdots & \mathrm{E}_{\mathrm{ft}}^{\mathrm{n}} & \mathrm{E}_{\mathrm{fb}}^{\mathrm{n}} & \mathbf{0} & \mathbf{0} & \cdots & \mathbf{E}_{\mathrm{ff}}^{\mathrm{n}}
\end{array}\right]\left\{\begin{array}{c}
\mathbf{u}_{\mathrm{t}}^{1} \\
\mathbf{u}_{\mathrm{b}}^{1} \\
\mathbf{u}_{\mathrm{t}}^{2} \\
\mathbf{u}_{\mathrm{b}}^{2} \\
\vdots \\
\mathbf{u}_{\mathrm{t}}^{\mathrm{n}} \\
\mathbf{u}_{\mathrm{b}}^{\mathrm{n}} \\
\mathbf{u}_{\mathrm{f}}^{1} \\
\mathbf{u}_{\mathrm{f}}^{2} \\
\vdots \\
\mathbf{u}_{\mathrm{f}}^{\mathrm{n}}
\end{array}\right\}=\left\{\begin{array}{c}
\mathbf{p}_{\mathrm{f}}^{1} \\
\mathbf{p}_{\mathrm{f}}^{2} \\
\vdots \\
\mathbf{p}_{\mathrm{f}}^{\mathrm{n}}
\end{array}\right\}
$$

$\mathrm{Na}$ igualdade 4.49, as equações foram organizadas de forma a deixar todos os deslocamentos referentes aos fustes por último no vetor de deslocamentos.

O passo seguinte é relacionar as equações 4.48 e 4.49 pelas condições de equilíbrio representadas pela relação 4.46. O procedimento consiste em igualar cada linha da equação 4.48 à sua correspondente da equação 4.49 multiplicada por (-1). Em seguida, isola-se no mesmo lado da igualdade da expressão resultante todos os termos relacionados aos deslocamentos de fuste. Considerando uma estaca $j$ genérica, escreve-se a equação:

$$
\begin{aligned}
& {\left[\mathbf{S}_{\mathrm{fjt}}^{\mathrm{i}}\right]\left\{\mathbf{u}_{\mathbf{t}}^{\mathrm{i}}\right\}+\left[\mathbf{S}_{\mathrm{fjb}}^{\mathrm{i}}\right]\left\{\mathbf{u}_{\mathbf{b}}^{\mathrm{i}}\right\}+\left[\mathbf{S}_{\mathrm{fjf} \mathbf{i}}^{\mathrm{i}}\right]\left\{\mathbf{u}_{\mathbf{f}}^{\mathbf{1}}\right\}+\cdots+\left[\mathbf{S}_{\mathrm{fjfn}}^{\mathrm{i}}\right]\left\{\mathbf{u}_{\mathbf{f}}^{\mathbf{n}}\right\}=} \\
& -\left[\mathbf{E}_{\mathrm{ft}}^{\mathbf{j}}\right]\left\{\mathbf{u}_{\mathrm{t}}^{\mathbf{j}}\right\}-\left[\mathbf{E}_{\mathrm{fb}}^{\mathbf{j}}\right]\left\{\mathbf{u}_{\mathrm{b}}^{\mathbf{j}}\right\}-\left[\mathbf{E}_{\mathrm{ff}}^{\mathbf{j}}\right]\left\{\mathbf{u}_{\mathbf{f}}^{\mathbf{j}}\right\}
\end{aligned}
$$

Passando para o lado esquerdo da igualdade todos os termos relacionados aos deslocamentos de fuste, chega-se à expressão:

$$
\begin{aligned}
& {\left[\mathbf{S}_{\mathrm{fjf} 1}^{\mathbf{i}}\right]\left\{\mathbf{u}_{\mathbf{f}}^{\mathbf{1}}\right\}+\cdots+\left[\overline{\mathbf{S}}_{\mathbf{f j f j}}\right]\left\{\mathbf{u}_{\mathbf{f}}^{\mathbf{j}}\right\}+\cdots+\left[\mathbf{S}_{\mathrm{fjfn}}^{\mathbf{i}}\right]\left\{\mathbf{u}_{\mathbf{f}}^{\mathbf{n}}\right\}=} \\
& -\left[\mathbf{S}_{\mathbf{f j t}}^{\mathbf{i}}\right]\left\{\mathbf{u}_{\mathbf{t}}^{\mathbf{i}}\right\}-\left[\mathbf{S}_{\mathbf{f j b}}^{\mathbf{i}}\right]\left\{\mathbf{u}_{\mathrm{b}}^{\mathbf{i}}\right\}-\left[\mathbf{E}_{\mathbf{f t}}^{\mathbf{j}}\right]\left\{\mathbf{u}_{\mathbf{t}}^{\mathbf{j}}\right\}-\left[\mathbf{E}_{\mathrm{fb}}^{\mathbf{j}}\right]\left\{\mathbf{u}_{\mathbf{b}}^{\mathbf{j}}\right\}
\end{aligned}
$$

em que a matriz $\overline{\mathbf{S}}_{\mathrm{fjfj}}$ é resultado da soma das matrizes $\mathbf{S}_{\mathrm{fjfj}}^{\mathbf{i}}$ e $\mathbf{E}_{\mathrm{ff}}^{\mathbf{j}}$. A equação 4.51 
escrita matricialmente é:

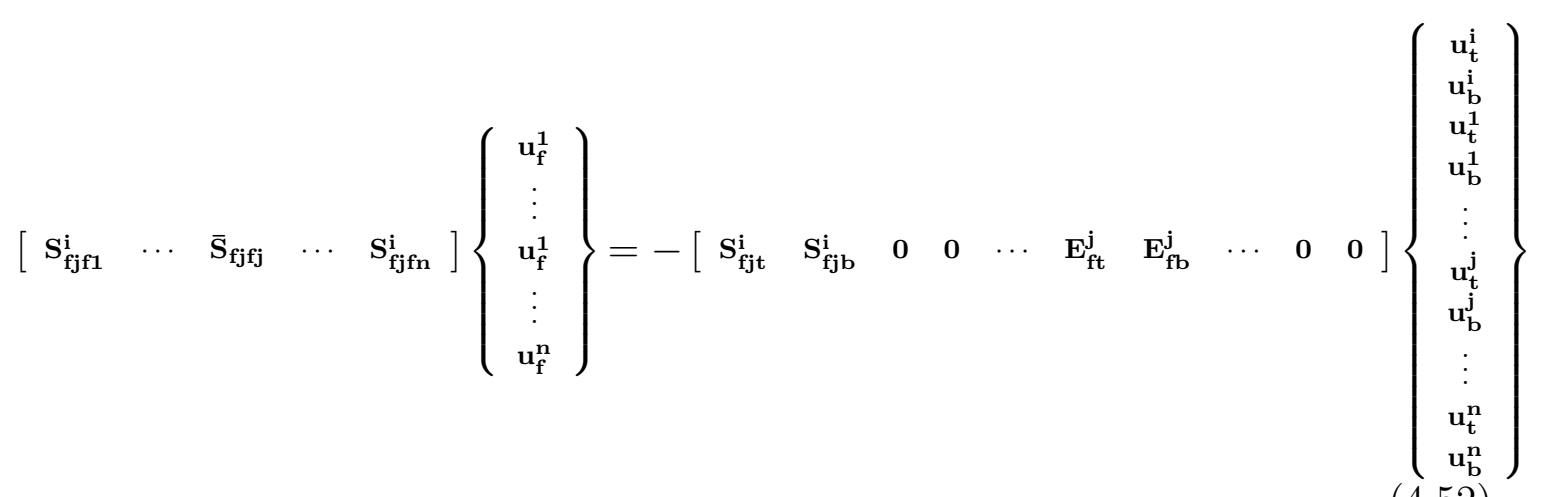

$\mathrm{O}$ conjunto de todas as equações 4.52, escritas para $\mathrm{j}$ de 1 até $n$, pode ser organizado em uma única equação. Esta equação é:

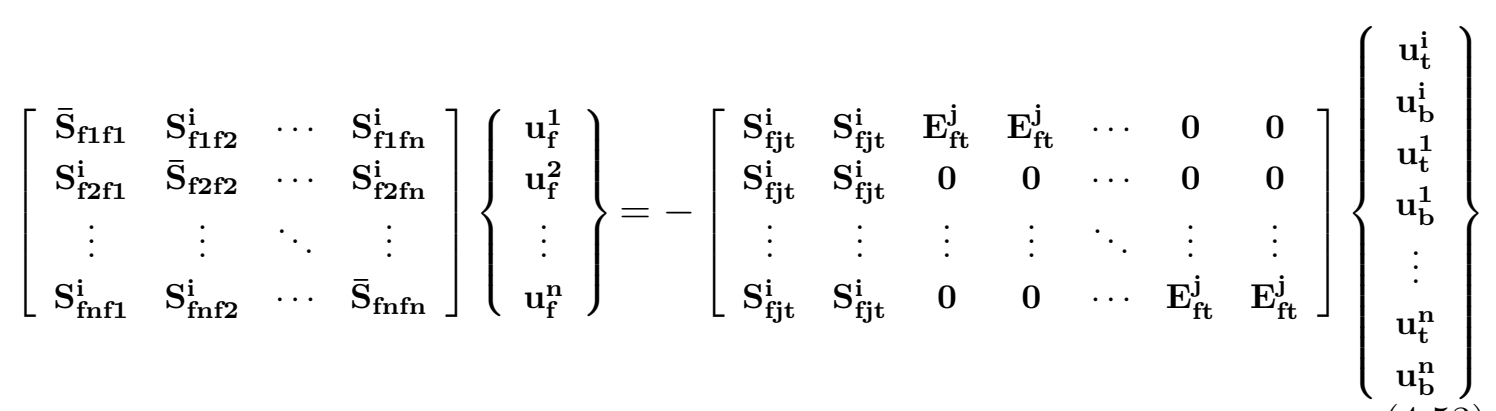

Para isolar os deslocamentos referentes aos fustes das estacas, deve-se inverter a matriz à equerda da igualdade da equação 4.53 e multiplicar o resultado a ambos os lados da equação. Assim, chega-se ao sistema de equações:

$$
\left\{\begin{array}{c}
\mathbf{u}_{\mathrm{f}}^{1} \\
\mathbf{u}_{\mathrm{f}}^{2} \\
\vdots \\
\mathbf{u}_{\mathrm{f}}^{\mathrm{n}}
\end{array}\right\}=\left[\begin{array}{cccc}
\overline{\mathbf{K}}_{11} & \overline{\mathbf{K}}_{12} & \cdots & \overline{\mathbf{K}}_{1(2 n+2)} \\
\overline{\mathbf{K}}_{21} & \overline{\mathbf{K}}_{22} & \cdots & \overline{\mathbf{K}}_{2(2 n+2)} \\
\vdots & \vdots & \ddots & \vdots \\
\overline{\mathbf{K}}_{(2 \mathbf{n}+2) 1} & \overline{\mathbf{K}}_{(2 n+2) 2} & \cdots & \overline{\mathbf{K}}_{(2 n+2)(2 n+2)}
\end{array}\right]\left\{\begin{array}{c}
u_{\mathrm{t}}^{\mathrm{i}} \\
u_{\mathrm{b}}^{\mathrm{i}} \\
\mathbf{u}_{\mathrm{t}}^{1} \\
\mathbf{u}_{\mathrm{b}}^{1} \\
\vdots \\
\mathbf{u}_{\mathrm{t}}^{\mathrm{n}} \\
\mathbf{u}_{\mathrm{b}}^{\mathrm{n}}
\end{array}\right\}
$$

Com o sistema 4.54, conclui-se a tarefa de escrever os deslocamentos dos fustes de todas as estacas em função dos nós de topo e base da camada $i$ e das estacas. Para obter uma parte do sistema de equações final, deve-se escrever isoladamente as 
duas primeiras linhas da equação 4.47. Isto fornece:

$$
\left[\begin{array}{cccccc}
S_{t t}^{i} & S_{t b}^{i} & S_{t f 1}^{i} & S_{t f 2}^{i} & \cdots & S_{t f n}^{i} \\
S_{b t}^{i} & S_{b b}^{i} & S_{b f 1}^{i} & S_{b f 2}^{i} & \cdots & S_{b f n}^{i}
\end{array}\right]\left\{\begin{array}{c}
u_{t}^{i} \\
u_{b}^{i} \\
u_{f}^{1} \\
u_{f}^{2} \\
\vdots \\
u_{f}^{n}
\end{array}\right\}=\left\{\begin{array}{c}
p_{t}^{i} \\
p_{b}^{i}
\end{array}\right\}
$$

Pode-se substituir a equação 4.54 na equação 4.55, eliminando dela os deslocamentos de fuste. O resultado é a seguinte expressão:

$$
\left[\begin{array}{cccc}
K_{11}^{i} & K_{12}^{i} & \cdots & K_{1(2 n+2)}^{i} \\
K_{21}^{i} & K_{22}^{i} & \cdots & K_{2(2 n+2)}^{i}
\end{array}\right]\left\{\begin{array}{c}
u_{t}^{i} \\
u_{b}^{i} \\
u_{t}^{1} \\
u_{b}^{1} \\
u_{t}^{2} \\
u_{b}^{2} \\
\vdots \\
u_{t}^{n} \\
u_{b}^{n}
\end{array}\right\}=\left\{\begin{array}{c}
p_{t}^{i} \\
p_{b}^{i}
\end{array}\right\}
$$

A expressão 4.56 corresponde às duas primeiras linhas da equação final. Podese chegar às demais linhas a partir das duas primeiras linhas da equação 4.44 em conjunto com a equação 4.54. As duas primeiras linhas de 4.44 isoladas são:

$$
\left[\begin{array}{lll}
E_{t t}^{j} & E_{t b}^{j} & E_{t f}^{j} \\
E_{b t}^{j} & E_{b b}^{j} & E_{b f}^{j}
\end{array}\right]\left\{\begin{array}{c}
u_{t}^{j} \\
u_{b}^{j} \\
u_{f}^{j}
\end{array}\right\}=\left\{\begin{array}{c}
p_{t}^{j} \\
p_{b}^{j}
\end{array}\right\}
$$

Como se está analisando uma estaca $j$, busca-se a linha $j$ da equação 4.54 . Esta é:

$$
\begin{aligned}
&\left\{\mathbf{u}_{\mathbf{f}}^{\mathbf{j}}\right\}=\left[\overline{\mathbf{K}}_{\mathbf{j} 1}\right]\left\{\mathbf{u}_{\mathbf{t}}^{\mathbf{i}}\right\}+\left[\overline{\mathbf{K}}_{\mathbf{j} \mathbf{2}}\right]\left\{\mathbf{u}_{\mathbf{b}}^{\mathbf{i}}\right\}+\left[\overline{\mathbf{K}}_{\mathbf{j} \mathbf{3}}\right]\left\{\mathbf{u}_{\mathbf{t}}^{\mathbf{1}}\right\}+\left[\overline{\mathbf{K}}_{\mathbf{j} 4}\right]\left\{\mathbf{u}_{\mathbf{b}}^{\mathbf{1}}\right\}+\cdots \\
&+\left[\overline{\mathbf{K}}_{\mathbf{j}(\mathbf{2 n}+\mathbf{1})}\right]\left\{\mathbf{u}_{\mathbf{t}}^{\mathbf{n}}\right\}+\left[\overline{\mathbf{K}}_{\mathbf{j}(\mathbf{2 n}+\mathbf{2})}\right]\left\{\mathbf{u}_{\mathbf{b}}^{\mathbf{n}}\right\}
\end{aligned}
$$

Substituindo a equação $4.58 \mathrm{em} 4.57$, obtém-se duas linhas da equação final. Isto 
é:

$$
\left[\begin{array}{cccc}
K_{(2 \mathbf{j}+1) 1}^{\mathrm{i}} & \mathbf{K}_{(2 \mathbf{j}+1) 2}^{\mathrm{i}} & \cdots & \mathbf{K}_{(2 \mathbf{j}+1)(2 n+2)}^{\mathrm{i}} \\
\mathbf{K}_{(2 \mathbf{2}+2) 1}^{\mathrm{i}} & \mathbf{K}_{(2 \mathbf{j}+2) 2}^{\mathrm{i}} & \cdots & \mathbf{K}_{(2 \mathbf{j}+2)(2 n+2)}^{\mathrm{i}}
\end{array}\right]\left\{\begin{array}{c}
\mathbf{u}_{\mathrm{t}}^{\mathrm{i}} \\
\mathbf{u}_{\mathrm{b}}^{\mathrm{i}} \\
\mathbf{u}_{\mathrm{t}}^{1} \\
\mathbf{u}_{\mathrm{b}}^{1} \\
\vdots \\
\mathbf{u}_{\mathrm{t}}^{\mathrm{n}} \\
\mathbf{u}_{\mathrm{b}}^{\mathrm{n}}
\end{array}\right\}=\left\{\begin{array}{c}
\mathbf{p}_{\mathrm{t}}^{\mathrm{j}} \\
\mathbf{p}_{\mathrm{b}}^{\mathrm{j}}
\end{array}\right\}
$$

A equação 4.59 deve ser escrita para $j$ de 1 até $n$. Estas equações em conjunto com a equação 4.56 compõem o sistema final de equações, que é o objetivo desta seção. Este sistema é:

$$
\left[\begin{array}{cccc}
K_{11}^{i} & K_{12}^{i} & \cdots & K_{1(2 n+2)}^{i} \\
K_{21}^{i} & K_{22}^{i} & \cdots & K_{2(2 n+2)}^{i} \\
\vdots & \vdots & \ddots & \vdots \\
K_{(2 n+2) 1}^{i} & K_{(2 n+2) 1}^{i} & \cdots & K_{(2 n+2)(2 n+2)}^{i}
\end{array}\right]\left\{\begin{array}{c}
u_{t}^{i} \\
u_{b}^{i} \\
u_{t}^{1} \\
u_{b}^{1} \\
\vdots \\
u_{t}^{n} \\
u_{b}^{n}
\end{array}\right\}=\left\{\begin{array}{c}
p_{t}^{i} \\
p_{b}^{i} \\
p_{t}^{1} \\
p_{b}^{1} \\
\vdots \\
p_{t}^{n} \\
p_{b}^{n}
\end{array}\right\}
$$

A partir da equação 4.60 torna-se possível aplicar o Método da Rigidez Sucessiva (MRS) às diferentes camadas, podendo estas conter um número qualquer de estacas cruzando-as. O procedimento iterativo de obtenção das matrizes $\hat{\mathbf{K}}^{\mathbf{i}}$ é igual ao empregado na seção 4.2, por isto não será refeito aqui. Entretanto, o cálculo dos valores de contorno na segunda etapa do MRS não pode ser feito da mesma forma devido aos contornos referentes aos contatos das camadas com as estacas. Portanto, o procedimento para a obtenção dos valores de contorno é descrito a seguir.

Após a conclusão da primeira etapa do MRS, referente à obtenção das matrizes $\hat{\mathbf{K}}^{\mathbf{i}}$ para todas as camadas, chega-se à seguinte equação:

$$
\left[\mathbf{K}^{\mathbf{n c}}\right]\left\{\mathbf{u}_{\mathbf{t}}^{\mathbf{n c}}\right\}=\left\{\mathbf{p}_{\mathbf{t}}^{\mathbf{n c}}\right\}
$$

em que nc é o número de camadas. Esta equação relaciona os deslocamentos $\mathbf{u}_{\mathbf{t}}^{\mathbf{n c}}$ na superfície livre do solo com as forças $\mathbf{p}_{\mathbf{t}}^{\mathbf{n c}}$ também da superfície, levando em conta a influência de todas as camadas de solo e todos os segmentos de estaca contidos nelas. Pelas hipóteses assumidas ao início da seção 4.2, o vetor $\mathbf{p}_{\mathbf{t}}^{\mathbf{n c}}$ é conhecido, o que torna possível encontrar o vetor $\mathbf{u}_{\mathbf{t}}^{\mathbf{n c}}$ a partir da equação 4.61 .

Após determinar os deslocamentos na superfície livre, escreve-se a equação 4.60 
para a camada nc, ou seja:

$$
\left[\begin{array}{cccc}
\mathbf{K}_{11}^{\mathrm{nc}} & \mathbf{K}_{12}^{\mathrm{nc}} & \cdots & \mathbf{K}_{1(2 n+2)}^{\mathrm{nc}} \\
\mathbf{K}_{21}^{\mathrm{nc}} & \mathbf{K}_{22}^{\mathrm{nc}} & \cdots & \mathbf{K}_{\mathbf{2}(2 \mathbf{n}+2)}^{\mathrm{c}} \\
\vdots & \vdots & \ddots & \vdots \\
\mathbf{K}_{(2 \mathbf{2 n}+2) 1}^{\mathrm{nc}} & \mathbf{K}_{(2 \mathbf{2 n}+2) 1}^{\mathrm{nc}} & \cdots & \mathbf{K}_{(2 \mathbf{2 n}+2)(2 n+2)}^{\mathrm{nc}}
\end{array}\right]\left\{\begin{array}{c}
\mathbf{u}_{\mathrm{t}}^{\mathrm{nc}} \\
\mathbf{u}_{\mathrm{b}}^{\mathrm{nc}} \\
\mathbf{u}_{\mathrm{t}}^{1} \\
\mathbf{u}_{\mathrm{b}}^{1} \\
\vdots \\
\mathbf{u}_{\mathrm{t}}^{\mathrm{n}} \\
\mathbf{u}_{\mathrm{b}}^{\mathrm{n}}
\end{array}\right\}=\left\{\begin{array}{c}
\mathbf{p}_{\mathrm{t}}^{\mathrm{nc}} \\
\mathbf{p}_{\mathrm{b}}^{\mathrm{nc}} \\
\mathbf{p}_{\mathrm{t}}^{1} \\
\mathbf{p}_{\mathrm{b}}^{1} \\
\vdots \\
\mathbf{p}_{\mathrm{t}}^{\mathrm{n}} \\
\mathbf{p}_{\mathrm{b}}^{\mathrm{n}}
\end{array}\right\}
$$

A matriz à esquerda da igualdade já foi deduzida anteriormente. Como são conhecidos todos os valores de contorno referentes ao topo da camada nc, o número de incógnitas na equação 4.62 é igual ao número de equações. Após organizar o sistema, passando para o lado esquerdo todos os valores desconhecidos, torna-se possível resolvê-lo determinando todos os valores de contorno referentes à base da camanda nc. Com estes dados, pode-se calcular os valores de deslocamento e força no fuste de cada estaca. Para isto escreve-se, considerando uma estaca $j$ qualquer, a equação:

$$
\left[\begin{array}{lll}
E_{t t}^{j} & E_{t b}^{j} & E_{t f}^{j} \\
E_{b t}^{j} & E_{b b}^{j} & E_{b f}^{j} \\
E_{f t}^{j} & E_{b t}^{j} & E_{f f}^{j}
\end{array}\right]\left\{\begin{array}{c}
u_{t}^{j} \\
u_{b}^{j} \\
u_{f}^{j}
\end{array}\right\}=\left\{\begin{array}{c}
p_{t}^{j} \\
p_{b}^{j} \\
p_{f}^{j}
\end{array}\right\}
$$

A matriz à esquerda da igualdade é conhecida, assim como os termos $\mathbf{u}_{\mathbf{t}}^{\mathbf{j}}, \mathbf{u}_{\mathbf{b}}^{\mathbf{j}}, \mathbf{p}_{\mathbf{t}}^{\mathbf{j}}$ e $\mathbf{p}_{\mathbf{b}}^{\mathbf{j}}$. Como a equação 4.63 tem duas incógnitas e três equações, pode-se determinar os valores desconhecidos escrevendo duas das esquações. A primeira linha de 4.63 fornece:

$$
\left[\mathbf{E}_{\mathbf{t t}}^{\mathbf{j}}\right]\left\{\mathbf{u}_{\mathbf{t}}^{\mathbf{j}}\right\}+\left[\mathbf{E}_{\mathbf{t b}}^{\mathbf{j}}\right]\left\{\mathbf{u}_{\mathbf{b}}^{\mathbf{j}}\right\}+\left[\mathbf{E}_{\mathbf{t f}}^{\mathbf{j}}\right]\left\{\mathbf{u}_{\mathbf{f}}^{\mathbf{j}}\right\}=\left\{\mathbf{p}_{\mathbf{t}}^{\mathbf{j}}\right\}
$$

Pode-se determinar o termo $\mathbf{u}_{\mathbf{f}}^{\mathbf{j}}$ isolando-o na equação 4.64. Ou seja:

$$
\left\{\mathbf{u}_{\mathbf{f}}^{\mathbf{j}}\right\}=\left[\mathbf{E}_{\mathbf{t f}}^{\mathbf{j}}\right]^{-\mathbf{1}}\left(\left\{\mathbf{p}_{\mathbf{t}}^{\mathbf{j}}\right\}-\left[\mathbf{E}_{\mathbf{t t}}^{\mathbf{j}}\right]\left\{\mathbf{u}_{\mathbf{t}}^{\mathbf{j}}\right\}-\left[\mathbf{E}_{\mathbf{t b}}^{\mathbf{j}}\right]\left\{\mathbf{u}_{\mathbf{b}}^{\mathbf{j}}\right\}\right)
$$

Sendo $\mathbf{u}_{\mathbf{f}}^{\mathbf{j}}$ conhecido, o termo $\mathbf{p}_{\mathbf{f}}^{\mathbf{j}}$ pode ser calculado diretamente da terceira linha de 4.63 , isto é:

$$
\left\{\mathbf{p}_{\mathbf{f}}^{\mathbf{j}}\right\}=\left[\mathbf{E}_{\mathrm{ft}}^{\mathbf{j}}\right]\left\{\mathbf{u}_{\mathbf{t}}^{\mathbf{j}}\right\}+\left[\mathbf{E}_{\mathrm{fb}}^{\mathbf{j}}\right]\left\{\mathbf{u}_{\mathbf{b}}^{\mathbf{j}}\right\}+\left[\mathbf{E}_{\mathrm{ff}}^{\mathbf{j}}\right]\left\{\mathbf{u}_{\mathbf{f}}^{\mathbf{j}}\right\}
$$

Por meio das relações 4.65 e 4.66 é possível obter os valores de contorno referentes aos fustes de todas as estacas, com referência nas estacas. Caso se queira determinar os deslocamentos e forças com referência na camada nc, basta aplicar as seguintes igualdades:

$$
\left\{\mathbf{u}_{\mathrm{fj}}^{\mathrm{i}}\right\}=\left\{\mathbf{u}_{\mathbf{f}}^{\mathrm{j}}\right\}
$$


$\mathrm{e}$

$$
\left\{\mathbf{p}_{\mathrm{fj}}^{\mathrm{i}}\right\}=-\left\{\mathbf{p}_{\mathbf{f}}^{\mathrm{j}}\right\}
$$

que representam as condições de compatibilidade de deslocamentos (relação 4.67) e equilíbrio de forças (relação 4.68), existentes nos contatos entre as estacas e a camada.

Os únicos valores de contorno da camada nc que ainda não foram determinados são aqueles referentes aos contornos laterais da camada, os deslocamentos $\mathbf{u}_{1}^{\text {nc }}$ e as forças $\mathbf{p}_{1}^{\mathbf{n c}}$. Pelas hipóteses assumidas no início da seção 4.2, $\mathbf{u}_{1}^{\mathbf{n c}}$ é igual a zero. Desta forma, o único valor de contorno ainda incógnito é o termo $\mathbf{p}_{\mathbf{l}}^{\mathbf{n c}}$. Para determinar este valor escreve-se, para a camada nc, a equação:

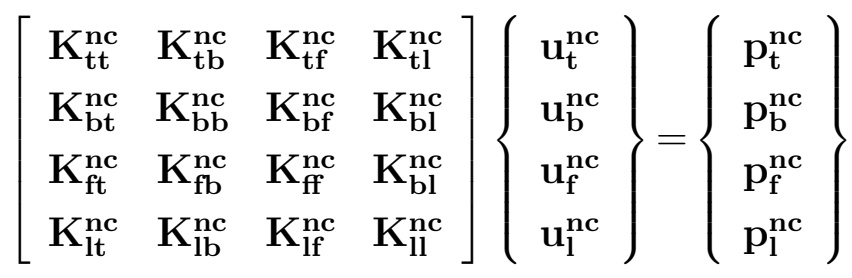

A equação 4.69 pode ser originada motando a equação 4.40 para a camada nc, ivertendo a matriz $\mathbf{G}^{\mathbf{n c}}$ e multiplicando-a ao lado esquerdo da equação. Os vetores $\mathbf{u}_{\mathbf{f}}^{\mathbf{n c}}$ e $\mathbf{p}_{\mathbf{f}}^{\mathbf{n c}}$ contêm os deslocamentos e forças referentes a todos os fustes de todas as estacas. Como pode ser observado, a única incógnita na equação 4.69 é o termo $\mathbf{p}_{\mathbf{l}}^{\mathbf{n c}}$. Este pode ser determinado diretamente da última linha da equação 4.69, ou seja:

$$
\left\{\mathbf{p}_{\mathbf{l}}^{\mathbf{n c}}\right\}=\left[\mathbf{K}_{\mathbf{l t}}^{\mathbf{n c}}\right]\left\{\mathbf{u}_{\mathbf{t}}^{\mathbf{n c}}\right\}+\left[\mathbf{K}_{\mathbf{l b}}^{\mathbf{n c}}\right]\left\{\mathbf{u}_{\mathbf{b}}^{\mathbf{n c}}\right\}+\left[\mathbf{K}_{\mathbf{l f}}^{\mathbf{n c}}\right]\left\{\mathbf{u}_{\mathbf{f}}^{\mathbf{n c}}\right\}+\left[\mathbf{K}_{\mathbf{l l}}^{\mathbf{n c}}\right]\left\{\mathbf{u}_{\mathbf{l}}^{\mathbf{n c}}\right\}
$$

Assim, ficam determinados todos os valores de contorno referentes à camada nc e aos segmentos de estaca contidos nela. Concluídos os cálculos referentes à camada nc, parte-se para a camada nc - 1. Para esta, escreve-se:

$$
\left[\begin{array}{cccc}
K_{11}^{n c-1} & K_{12}^{n c-1} & \cdots & K_{1(2 n+2)}^{n c-1} \\
K_{21}^{n c-1} & K_{22}^{n c-1} & \cdots & K_{2(2 n+2)}^{n c-1} \\
\vdots & \vdots & \ddots & \vdots \\
K_{(2 n+2) 1}^{n c-1} & K_{(2 n+2) 1}^{n c-1} & \cdots & K_{(2 n+2)(2 n+2)}^{n c-1}
\end{array}\right]\left\{\begin{array}{c}
u_{t}^{n c-1} \\
u_{b}^{n c-1} \\
u_{t}^{1} \\
u_{b}^{1} \\
\vdots \\
u_{t}^{n} \\
u_{b}^{n}
\end{array}\right\}=\left\{\begin{array}{c}
p_{t}^{n c-1} \\
p_{b}^{n c-1} \\
p_{t}^{1} \\
p_{b}^{1} \\
\vdots \\
p_{t}^{n} \\
p_{b}^{n}
\end{array}\right\}
$$

A matriz à esquerda da igualdade é conhecida. Pode-se facilmente determinar também todos os valores de contorno referentes ao topo da camada $\mathbf{n c}-\mathbf{1}$ aplicando condições de equilíbrio e compatibilidade entre as camadas nc e nc-1. A partir 
destas condições, escreve-se as igualdades:

$$
\left\{\mathbf{u}_{\mathbf{t}}^{\mathrm{nc}-1}\right\}=\left\{\mathbf{u}_{\mathbf{b}}^{\mathrm{nc}}\right\}
$$

e

$$
\left\{\mathbf{p}_{\mathbf{t}}^{\mathbf{n c}-1}\right\}=-\left\{\mathbf{p}_{\mathbf{b}}^{\mathbf{n c}}\right\}
$$

Repetindo os procedimentos realizados entre as equações 4.62 e 4.71 para a camada nc $\mathbf{- 1}$, determina-se todos os valores de contorno referentes a esta camada e aos segmentos de estaca contidos nela. Este cálcutos são então refeitos para todas as camadas inferiores, determinando todos os valores de contorno de todos os subdomínios do problema. Desta forma, torna-se possível calcular deslocamentos e tensões em pontos internos a qualquer um destes subdomínios.

Com isto, fica concluída toda a teoria utilizada em ALMEIDA 2003a) na simulação do maciço de solos estratificado em camadas e com um número qualquer de estacas.

\subsection{Considerações finais}

Neste capítulo foi empregada uma extensão do Método da Rigidez Sucessiva, apresentado em MAIER \& NOVATI (1987), com o objetivo de obter uma matriz que representa a influência de todas as camadas de solo e de todas as estacas contidas nestes meios.

O MRS foi aplicado inicialmente ao solo tridimensional sem estacas, para melhor entendimento de seu funcionamento. Considerando uma camada genérica como um sólido tridimensional, aplicou-se a formulação do MEC para chegar às matrizes $H$ e $G$ desta camada. A partir de condições de compatibilidade de deslocamentos e equilíbrio de forças, foi obtida uma equação que representa a influência da camada em função de seus nós de topo e base. Tomando a camada em contato com a superfície de deslocamento nulo, chegou-se a outra equação que computa toda a influência desta camada em função apenas dos nós localizados em seu topo. Uma nova aplicação das condições de compatibilidade de deslocamentos e equilíbrio de forças resultou nesta mesma equação para a camada superior. Repetindo este procedimento interativamente atinge-se a camada da superfície, concluindo com um sistema de equações que relaciona os deslocamentos nos nós de topo desta camada às forças nestes mesmos nós por meio de uma matriz que tem computadas as influências de todas as camadas. Resolvendo-se o sistema, obtém-se os deslocamentos na superfície livre do solo. Inicia-se então a segunda etapa do MRS, calculando os valores de contorno de todas as camadas de cima para baixo. Por fim, torna-se possível determinar deslocamentos e tensões em nós internos a qualquer camada. 
Em seguida, na seção 4.3, foi demonstrado como incluir a influência das estacas na formulação do MRS. Chega-se a uma matriz que computa a influência de uma camada qualquer cruzada por um número qualquer de estacas, relacionando os deslocamentos de topo e base desta camada às forças de topo e base. Utilizando esta matriz como ponto de partida torna-se possível aplicar o MRS da mesma forma que na seção 4.2 , obtendo todos os valores de contorno referentes às camadas e estacas. 


\section{Capítulo 5}

\section{Interação do solo com estrutura}

\subsection{Introdução}

Este capítulo trata da interação do solo com dois tipos de estruturas. O primeiro são blocos tridimensionais modelados pelo MEC, e o segundo é um edifício tridimensional modelado pelo MEF. Ao final do capítulo é descrito como este edifício é apoiado sobre os blocos, compondo o conjunto solo/blocos/edifício.

A interação do solo com um bloco, ambos modelados pelo MEC, é tratada inicialmente. Este caso é um problema de subregiões do MEC, e pode ser resolvido aplicando condições de compatibilidade de deslocamentos e equilíbrio de forças nos nós de contato entre os dois meios. Na sequência, é descrita a teoria utilizada no acoplamento entre um número qualquer de blocos e o solo.

O estudo da interação do solo com o edifício envolve domínios modelados por diferentes técnicas numéricas, sendo o solo simulado pelo MEC e o edifício pelo MEF. É possível acoplar estas formulações por meio das condições de compatibilidade e equilíbrio, no entanto o vetor de forças advindo do MEC deve ser compatibilizado antes de ser relacionado à formulação do MEF.

Representando o conjunto formado pelo solo e os blocos com uma única matriz de influência, torna-se possível acoplar o edifício a este sistema utilizando a mesma teoria empregada no acoplamento do solo sem blocos ao edifício.

\subsection{Acoplamento entre o solo e um bloco}

As influências das camadas de solo foram condensadas por meio do Método da Rigidez Sucessiva (MRS), conforme descrito no capítulo 4. O resultado deste procedimento foi um sistema de equações que relaciona os deslocamentos dos nós da superfície livre do solo com as forças prescritas nestes nós. Este sistema, mostrado 
na equação 4.61 , pode ser escrito como:

$$
\left[\mathbf{K}^{\mathbf{S}}\right]\left\{\mathbf{u}^{\mathbf{S}}\right\}=\left\{\mathbf{p}^{\mathbf{S}}\right\}
$$

O índice $\mathbf{S}$ indica que os vetores e matrizes foram originados da formulação feita para o solo. A matriz $\mathbf{K}^{\mathbf{S}}$, como foi visto no capítulo 4, contém a matriz de influência de todas as camadas de solo.

Considerando que o bloco é um sólido tridimensional, pode-se aplicar a ele a formulação do MEC descrita no capítulo 2. Assim sendo, pelo MEC, tem-se a equação 2.110 para o bloco. Esta equação é:

$$
\left[\mathbf{H}^{\mathrm{B}}\right]\left\{\mathbf{u}^{\mathrm{B}}\right\}=\left[\mathbf{G}^{\mathrm{B}}\right]\left\{\mathbf{p}^{\mathrm{B}}\right\}
$$

Para tornar as deduções mais práticas, é interessante obter uma equação similar à equação 5.1 para o bloco. Portanto, inverte-se a matriz $\mathbf{G}_{\mathbf{B}}$ e multiplica-se o resultado a ambos os lados da equação 5.2 . O resultado é a equação:

$$
\left[\mathbf{K}^{\mathbf{B}}\right]\left\{\mathbf{u}^{\mathbf{B}}\right\}=\left\{\mathbf{p}^{\mathbf{B}}\right\}
$$

com

$$
\left[\mathbf{K}^{\mathrm{B}}\right]=\left[\mathbf{G}^{\mathrm{B}}\right]^{-\mathbf{1}}\left[\mathbf{H}^{\mathrm{B}}\right]
$$

O acoplamento entre o solo e o bloco, ilustrado na figura 5.1, é realizado a partir das equações 5.1 e 5.3 .

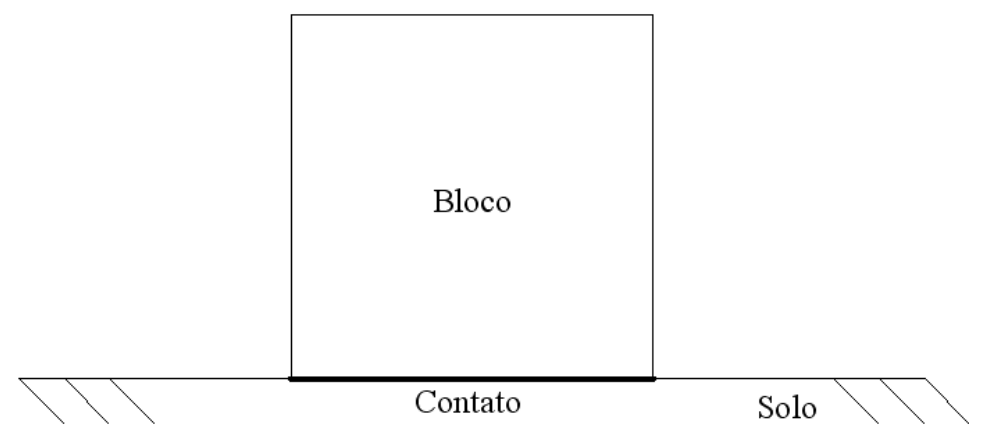

Figura 5.1: Conjunto formado pelo solo e o bloco

Primeiramente deve-se separar, nas equações 5.1] e 5.3, as parcelas referentes aos nós que pertencem ao contato entre os meios das parcelas referentes aos nós que não pertencem ao contato entre os meios. Indicando as parcelas pertencentes ao contato pelo índice subscrito $\mathbf{C}$ e as parcelas pertencentes às superfícies livres pelo índice 
subscrito L, obtém-se as seguintes equações:

$$
\begin{aligned}
& {\left[\begin{array}{ll}
K_{L L}^{S} & K_{L C}^{S} \\
K_{C L}^{S} & K_{C C}^{S}
\end{array}\right]\left\{\begin{array}{l}
u_{L}^{S} \\
u_{C}^{S}
\end{array}\right\}=\left\{\begin{array}{l}
p_{L}^{S} \\
p_{C}^{S}
\end{array}\right\}} \\
& {\left[\begin{array}{ll}
K_{L L}^{B} & K_{L C}^{B} \\
K_{C L}^{B} & K_{C C}^{B}
\end{array}\right]\left\{\begin{array}{l}
u_{L}^{B} \\
u_{C}^{B}
\end{array}\right\}=\left\{\begin{array}{l}
p_{L}^{B} \\
p_{C}^{B}
\end{array}\right\}}
\end{aligned}
$$

Para relacionar as equações 5.5 e 5.6 aplica-se, na superfície de contato entre os dois meios, condições de compatibilidade de deslocamentos e equilíbrio de forças. A compatibilidade de deslocamentos permite escrever as igualdades:

$$
\left\{\mathbf{u}_{\mathbf{C}}^{\mathbf{S}}\right\}=\left\{\mathbf{u}_{\mathbf{C}}^{\mathrm{B}}\right\}=\left\{\mathbf{u}_{\mathbf{C}}\right\}
$$

Aplicando as condições de compatibilidade de deslocamentos representadas por 5.7 nas equações 5.5 e 5.6 , chega-se às equações:

$$
\begin{aligned}
& {\left[\begin{array}{ll}
\mathbf{K}_{\mathbf{L L}}^{\mathrm{S}} & \mathbf{K}_{\mathbf{L C}}^{\mathrm{S}} \\
\mathbf{K}_{\mathbf{C L}}^{\mathrm{S}} & \mathbf{K}_{\mathbf{C C}}^{\mathrm{S}}
\end{array}\right]\left\{\begin{array}{l}
\mathbf{u}_{\mathbf{L}}^{\mathrm{S}} \\
\mathbf{u}_{\mathbf{C}}
\end{array}\right\}=\left\{\begin{array}{l}
\mathbf{p}_{\mathbf{L}}^{\mathrm{S}} \\
\mathbf{p}_{\mathbf{C}}^{\mathrm{S}}
\end{array}\right\}} \\
& {\left[\begin{array}{ll}
\mathbf{K}_{\mathbf{L L}}^{\mathrm{B}} & \mathrm{K}_{\mathrm{LC}}^{\mathrm{B}} \\
\mathbf{K}_{\mathrm{CL}}^{\mathrm{B}} & \mathbf{K}_{\mathrm{CC}}^{\mathrm{B}}
\end{array}\right]\left\{\begin{array}{l}
\mathbf{u}_{\mathbf{L}}^{\mathrm{B}} \\
\mathbf{u}_{\mathrm{C}}
\end{array}\right\}=\left\{\begin{array}{l}
\mathbf{p}_{\mathbf{L}}^{\mathrm{B}} \\
\mathbf{p}_{\mathrm{C}}^{\mathrm{B}}
\end{array}\right\}}
\end{aligned}
$$

Utilizando a partir deste ponto as equações 5.8 e 5.9, torna-se desnecessário recorrer novamente às igualdades 5.7. As equações 5.8 e 5.9 devem ser relacionadas para que os domínios sejam acoplados. Para isto impõe-se no contato condições de equilíbrio de forças, traduzidas pela igualdade:

$$
\left\{\mathbf{p}_{\mathbf{C}}^{\mathbf{S}}\right\}=-\left\{\mathbf{p}_{\mathbf{C}}^{\mathbf{B}}\right\}
$$

Para que a igualdade 5.10 possa ser utilizada escreve-se, a partir das equações 5.8 e 5.9, expressões que representem as forças no contato. Assim, a segunda linha de 5.8 fornece:

$$
\left[\mathbf{K}_{\mathbf{C L}}^{\mathbf{S}}\right]\left\{\mathbf{u}_{\mathbf{L}}^{\mathbf{S}}\right\}+\left[\mathbf{K}_{\mathbf{C C}}^{\mathbf{S}}\right]\left\{\mathbf{u}_{\mathbf{C}}\right\}=\left\{\mathbf{p}_{\mathbf{C}}^{\mathbf{S}}\right\}
$$

E a segunda linha de 5.9 gera a igualdade:

$$
\left[\mathbf{K}_{\mathbf{C L}}^{\mathrm{B}}\right]\left\{\mathbf{u}_{\mathbf{L}}^{\mathrm{B}}\right\}+\left[\mathbf{K}_{\mathbf{C C}}^{\mathrm{B}}\right]\left\{\mathbf{u}_{\mathbf{C}}\right\}=\left\{\mathbf{p}_{\mathbf{C}}^{\mathrm{B}}\right\}
$$

Por meio da igualdade 5.10 , é possível unir as expressões 5.11 e 5.12 em uma única. O resultado é a igualdade:

$$
\left[\mathbf{K}_{\mathbf{C L}}^{\mathbf{S}}\right]\left\{\mathbf{u}_{\mathbf{L}}^{\mathbf{S}}\right\}+\left[\mathbf{K}_{\mathbf{C C}}^{\mathbf{S}}\right]\left\{\mathbf{u}_{\mathbf{C}}\right\}=-\left[\mathbf{K}_{\mathbf{C L}}^{\mathbf{B}}\right]\left\{\mathbf{u}_{\mathbf{L}}^{\mathbf{B}}\right\}-\left[\mathbf{K}_{\mathbf{C C}}^{\mathbf{B}}\right]\left\{\mathbf{u}_{\mathbf{C}}\right\}
$$


Desenvolvendo a expressão 5.13, é possível isolar os deslocamentos do contato, uc. Assim:

$$
\begin{aligned}
& \left(\left[\mathbf{K}_{\mathbf{C C}}^{\mathbf{S}}\right]+\left[\mathbf{K}_{\mathbf{C C}}^{\mathbf{B}}\right]\right)\left\{\mathbf{u}_{\mathbf{C}}\right\}=-\left[\mathbf{K}_{\mathbf{C L}}^{\mathbf{S}}\right]\left\{\mathbf{u}_{\mathbf{L}}^{\mathbf{S}}\right\}-\left[\mathbf{K}_{\mathbf{C L}}^{\mathrm{B}}\right]\left\{\mathbf{u}_{\mathbf{L}}^{\mathbf{B}}\right\} \\
& \left\{\mathbf{u}_{\mathbf{C}}\right\}=-\left[\mathbf{K}_{\mathbf{C C}}\right]^{-\mathbf{1}}\left[\mathbf{K}_{\mathbf{C L}}^{\mathbf{S}}\right]\left\{\mathbf{u}_{\mathbf{L}}^{\mathbf{S}}\right\}-\left[\mathbf{K}_{\mathbf{C C}}\right]^{-\mathbf{1}}\left[\mathbf{K}_{\mathbf{C L}}^{\mathbf{B}}\right]\left\{\mathbf{u}_{\mathbf{L}}^{\mathbf{B}}\right\}
\end{aligned}
$$

com

$$
\left[\mathbf{K}_{\mathbf{C C}}\right]=\left(\left[\mathbf{K}_{\mathbf{C C}}^{\mathbf{S}}\right]+\left[\mathbf{K}_{\mathbf{C C}}^{\mathbf{B}}\right]\right)
$$

A expressão 5.15 relaciona os deslocamentos nos nós do contato aos deslocamentos nas superfícies livres do solo e do bloco. Analisando agora as equações relacionadas às forças na superfície livre do solo, escreve-se a primeira linha da equação 5.8 .

$$
\left[\mathbf{K}_{\mathbf{L L}}^{\mathbf{S}}\right]\left\{\mathbf{u}_{\mathbf{L}}^{\mathbf{S}}\right\}+\left[\mathbf{K}_{\mathbf{L C}}^{\mathbf{S}}\right]\left\{\mathbf{u}_{\mathbf{C}}\right\}=\left\{\mathbf{p}_{\mathbf{L}}^{\mathbf{S}}\right\}
$$

Substituindo a expressão 5.15 na relação 5.17 , chega-se à equação:

$$
\left[\mathbf{K}_{\mathbf{L L}}^{\mathbf{S}}\right]\left\{\mathbf{u}_{\mathbf{L}}^{\mathbf{S}}\right\}+\left[\mathbf{K}_{\mathbf{L C}}^{\mathbf{S}}\right]\left(-\left[\mathbf{K}_{\mathbf{C C}}\right]^{-\mathbf{1}}\left[\mathbf{K}_{\mathbf{C L}}^{\mathbf{S}}\right]\left\{\mathbf{u}_{\mathbf{L}}^{\mathbf{S}}\right\}-\left[\mathbf{K}_{\mathbf{C C}}\right]^{-\mathbf{1}}\left[\mathbf{K}_{\mathbf{C L}}^{\mathbf{B}}\right]\left\{\mathbf{u}_{\mathbf{L}}^{\mathbf{B}}\right\}\right)=\left\{\mathbf{p}_{\mathbf{L}}^{\mathbf{S}}\right\}
$$

A equação 5.18 deve ser desenvolvida de forma a isolar os deslocamentos ao lado direito da igualdade e as forças ao lado esquerdo. Efetuando os produtos de matrizes, conclui-se com a igualdade:

$$
\left[\mathbf{K}_{11}\right]\left\{\mathbf{u}_{\mathbf{L}}^{\mathbf{S}}\right\}+\left[\mathbf{K}_{12}\right]\left\{\mathbf{u}_{\mathbf{L}}^{\mathbf{B}}\right\}=\left\{\mathbf{p}_{\mathbf{L}}^{\mathbf{S}}\right\}
$$

Prosseguindo com as deduções, analisa-se agora as equações relacionadas às forças na superfície livre do bloco. A primeira linha da equação 5.9, referente a estas forças, é:

$$
\left[\mathbf{K}_{\mathbf{L L}}^{\mathbf{B}}\right]\left\{\mathbf{u}_{\mathbf{L}}^{\mathbf{B}}\right\}+\left[\mathbf{K}_{\mathbf{L C}}^{\mathbf{B}}\right]\left\{\mathbf{u}_{\mathbf{C}}\right\}=\left\{\mathbf{p}_{\mathbf{L}}^{\mathbf{B}}\right\}
$$

Substituindo a expressão 5.15 na equação 5.20 , obtém-se a igualdade:

$$
\left[\mathbf{K}_{\mathbf{L L}}^{\mathrm{B}}\right]\left\{\mathbf{u}_{\mathbf{L}}^{\mathrm{B}}\right\}+\left[\mathbf{K}_{\mathbf{L C}}^{\mathrm{B}}\right]\left(-\left[\mathbf{K}_{\mathbf{C C}}\right]^{-\mathbf{1}}\left[\mathbf{K}_{\mathbf{C L}}^{\mathbf{S}}\right]\left\{\mathbf{u}_{\mathbf{L}}^{\mathbf{S}}\right\}-\left[\mathbf{K}_{\mathbf{C C}}\right]^{-\mathbf{1}}\left[\mathbf{K}_{\mathbf{C L}}^{\mathrm{B}}\right]\left\{\mathbf{u}_{\mathbf{L}}^{\mathrm{B}}\right\}\right)=\left\{\mathbf{p}_{\mathbf{L}}^{\mathrm{B}}\right\}
$$

Desenvolvendo a equação 5.21 da mesma forma feita com a equação 5.18 , concluise com a igualdade:

$$
\left[\mathbf{K}_{\mathbf{2 1}}\right]\left\{\mathbf{u}_{\mathbf{L}}^{\mathbf{S}}\right\}+\left[\mathbf{K}_{\mathbf{2 2}}\right]\left\{\mathbf{u}_{\mathbf{L}}^{\mathrm{B}}\right\}=\left\{\mathbf{p}_{\mathbf{L}}^{\mathrm{B}}\right\}
$$

As equações 5.22 e 5.19 compõem o seguinte sistema de equações:

$$
\left[\begin{array}{ll}
K_{11} & K_{12} \\
K_{21} & K_{22}
\end{array}\right]\left\{\begin{array}{l}
u_{L}^{S} \\
u_{L}^{B}
\end{array}\right\}=\left\{\begin{array}{l}
p_{L}^{S} \\
p_{L}^{B}
\end{array}\right\}
$$


$\mathrm{Ou}$

$$
[\mathbf{K}]\{\mathbf{u}\}=\{\mathbf{p}\}
$$

Prescrevendo forças em todos os nós das superfícies livres do solo e do bloco, torna-se possível resolver o sistema de equações 5.23 . Sua solução leva aos deslocamentos nas superfícies livres do solo e do bloco. Determinados os deslocamentos nas superfícies livres, torna-se possível calcular os deslocamentos nos nós do contato a partir da expressão 5.15. Ou seja:

$$
\left\{\mathbf{u}_{\mathbf{C}}\right\}=-\left[\mathbf{K}_{\mathbf{C C}}\right]^{-\mathbf{1}}\left[\mathbf{K}_{\mathbf{C L}}^{\mathbf{S}}\right]\left\{\mathbf{u}_{\mathbf{L}}^{\mathbf{S}}\right\}-\left[\mathbf{K}_{\mathbf{C C}}\right]^{-1}\left[\mathbf{K}_{\mathbf{C L}}^{\mathrm{B}}\right]\left\{\mathbf{u}_{\mathbf{L}}^{\mathbf{B}}\right\}
$$

Depois de determinar todos os deslocamentos referentes ao bloco e à superfície livre do solo, pode-se calcular as forças nos nós do contato pela expressão 5.11 ou pela expressão 5.12 , isto é:

$$
\begin{aligned}
& {\left[\mathbf{K}_{\mathbf{C L}}^{\mathbf{S}}\right]\left\{\mathbf{u}_{\mathbf{L}}^{\mathbf{S}}\right\}+\left[\mathbf{K}_{\mathbf{C C}}^{\mathbf{S}}\right]\left\{\mathbf{u}_{\mathbf{C}}\right\}=\left\{\mathbf{p}_{\mathbf{C}}^{\mathbf{s}}\right\}} \\
& {\left[\mathbf{K}_{\mathbf{C L}}^{\mathbf{B}}\right]\left\{\mathbf{u}_{\mathbf{L}}^{\mathbf{B}}\right\}+\left[\mathbf{K}_{\mathbf{C C}}^{\mathbf{B}}\right]\left\{\mathbf{u}_{\mathbf{C}}\right\}=\left\{\mathbf{p}_{\mathbf{C}}^{\mathbf{B}}\right\}}
\end{aligned}
$$

As forças de contato obtidas pela igualdade 5.26 são iguais às forças de contato obtidas pela relação 5.27, porém com o sinal trocado. Isto acontece por causa da condição de equilíbrio imposta pela igualdade 5.10 , ou seja:

$$
\left\{\mathbf{p}_{\mathbf{C}}^{\mathbf{S}}\right\}=-\left\{\mathbf{p}_{\mathbf{C}}^{\mathrm{B}}\right\}
$$

\subsection{Acoplamento entre o solo e $N$ blocos}

No caso do acoplamento do solo com um único bloco, partiu-se da equação 5.3 . Nesta equação a matriz $\mathbf{K}^{\mathbf{B}}$ representa o bloco nas deduções subsequentes, que resultam na matriz $\mathbf{K}$ da equação 5.24. Caso exista mais de um bloco, uma forma de aproveitar estas mesmas deduções é obter uma matriz $\mathbf{K}^{\mathbf{B}}$ que represente todos os blocos definidos no problema. Pode-se então utilizar esta matriz na equação 5.3 e seguir com as demais deduções até a equação 5.24, representando o acoplamento entre um número qualquer de blocos e o solo. Existindo $N$ blocos, a equação equivalente a 5.3 que deve ser obtida é:

$$
\left[\mathbf{K}^{\mathrm{BN}}\right]\left\{\mathbf{u}^{\mathrm{BN}}\right\}=\left\{\mathbf{p}^{\mathrm{BN}}\right\}
$$

O vetor $\mathbf{u}^{\mathrm{BN}}$ contém os deslocamentos em todos os nós do contorno dos blocos, enquanto o vetor $\mathbf{p}^{\mathbf{B N}}$ contém as forças em todos os nós do contorno dos blocos. A matriz $\mathbf{K}^{\mathbf{B N}}$ relaciona estes dois vetores, e representa a rigidez dos blocos. Para 
facilitar o entendimento, inicialmente é apresentada a dedução da matriz $\mathbf{K}^{\mathbf{B N}}$ para $N$ igual a 2 para depois generalizar o raciocínio para qualquer $N$. Considerando dois blocos, a equação 5.3 pode ser escrita para ambos seguindo as instruções do início da seção 5.2. Ou seja:

$$
\left[\mathbf{K}^{\mathrm{B} 1}\right]\left\{\mathbf{u}^{\mathrm{B} 1}\right\}=\left\{\mathbf{p}^{\mathrm{B} 1}\right\}
$$

e

$$
\left[\mathbf{K}^{\mathrm{B} 2}\right]\left\{\mathbf{u}^{\mathrm{B} 2}\right\}=\left\{\mathbf{p}^{\mathrm{B} 2}\right\}
$$

Também na seção 5.2 , escreveu-se a equação 5.3 separando as parcelas referentes aos nós que pertencem ao contato entre o bloco e o solo das parcelas referentes aos nós que pertencem à superfície livre do bloco. O resultado foi a equação 5.6, que no caso das equações 5.30 e 5.31 se torna:

$$
\left[\begin{array}{ll}
\mathbf{K}_{\mathbf{L} 1}^{\mathrm{B}} & \mathbf{K}_{\mathbf{L C}}^{\mathrm{B} 1} \\
\mathbf{K}_{\mathbf{C L}}^{\mathrm{B} 1} & \mathbf{K}_{\mathbf{C C}}^{\mathrm{B} 1}
\end{array}\right]\left\{\begin{array}{l}
\mathbf{u}_{\mathbf{L}}^{\mathrm{B} 1} \\
\mathbf{u}_{\mathbf{C}}^{\mathrm{B} 1}
\end{array}\right\}=\left\{\begin{array}{l}
\mathbf{p}_{\mathbf{L}}^{\mathrm{B} 1} \\
\mathbf{p}_{\mathbf{C}}^{\mathrm{B} 1}
\end{array}\right\}
$$

e

$$
\left[\begin{array}{ll}
\mathbf{K}_{\mathrm{L}}^{\mathrm{B} 2} & \mathbf{K}_{\mathbf{L C}}^{\mathrm{B} 2} \\
\mathbf{K}_{\mathrm{CL}}^{\mathrm{B} 2} & \mathbf{K}_{\mathbf{C C}}^{\mathrm{B} 2}
\end{array}\right]\left\{\begin{array}{l}
\mathbf{u}_{\mathrm{L}}^{\mathrm{B} 2} \\
\mathbf{u}_{\mathbf{C}}^{\mathrm{B} 2}
\end{array}\right\}=\left\{\begin{array}{l}
\mathbf{p}_{\mathbf{L}}^{\mathrm{B} 2} \\
\mathbf{p}_{\mathbf{C}}^{\mathrm{B} 2}
\end{array}\right\}
$$

A matriz $\mathbf{K}^{\mathbf{B N}}$ procurada pode ser obtida a partir das equações 5.32 e 5.33 . É importante observar que, para que as deduções compreendidas entre as equações 5.3 e 5.24 sejam válidas, nos vetores $\mathbf{u}^{\mathbf{B N}}$ e $\mathbf{p}^{\mathbf{B N}}$ as parcelas referentes aos nós do contato dos dois blocos com o solo devem estar agrupadas na parte inferior dos vetores, enquanto as parcelas referentes aos nós das superfícies livres dos dois blocos devem estar agrupadas na parte superior dos vetores. Isto significa que o vetor de deslocamentos $\mathbf{u}^{\mathbf{B N}}$ deve ser:

$$
\left\{\mathbf{u}^{\mathrm{BN}}\right\}=\left\{\begin{array}{c}
\mathbf{u}_{\mathbf{L}}^{\mathrm{B} 1} \\
\mathbf{u}_{\mathbf{L}}^{\mathrm{B} 2} \\
\mathbf{u}_{\mathbf{C}}^{\mathrm{B} 1} \\
\mathbf{u}_{\mathbf{C}}^{\mathrm{B} 2}
\end{array}\right\}
$$

O vetor de forças $\mathbf{p}^{\mathbf{B N}}$, análogo ao vetor $\mathbf{u}^{\mathbf{B N}}$ considerado na igualdade 5.34 , é dado por:

$$
\left\{\mathbf{p}^{\mathrm{BN}}\right\}=\left\{\begin{array}{l}
\mathbf{p}_{\mathbf{L}}^{\mathrm{B} 1} \\
\mathbf{p}_{\mathbf{L}}^{\mathrm{B} 2} \\
\mathbf{p}_{\mathrm{C} 1}^{\mathrm{B} 1} \\
\mathbf{p}_{\mathrm{C}}^{\mathrm{B} 2}
\end{array}\right\}
$$

A partir das expressões 5.32, 5.33, 5.34 e 5.35, é possível montar a seguinte 
equação:

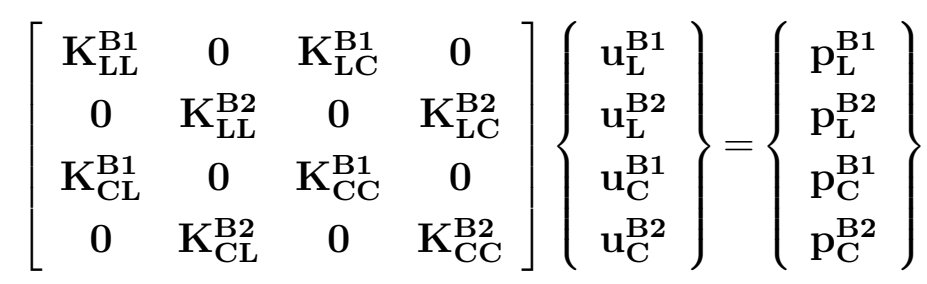

$\mathrm{Ou}$

$$
\left[\mathbf{K}^{\mathrm{BN}}\right]\left\{\mathbf{u}^{\mathrm{BN}}\right\}=\left\{\mathbf{p}^{\mathrm{BN}}\right\}
$$

Como não existe contato entre os blocos, as parcelas referentes aos nós de cada bloco são independentes umas das outras. Isto pode ser observado na matriz $\mathbf{K}^{\mathbf{B N}}$ pelos trechos de zeros que aparecem, nunca ocorrendo o produto de uma submatriz de um bloco por um deslocamento de outro.

Por fim, torna-se possível utilizar a equação 5.6 da seção 5.2 por meio das seguintes associações:

$$
\begin{gathered}
{\left[\mathbf{K}_{\mathbf{L L}}^{\mathrm{B}}\right]=\left[\begin{array}{cc}
\mathbf{K}_{\mathbf{L L}}^{\mathrm{B} 1} & \mathbf{0} \\
\mathbf{0} & \mathbf{K}_{\mathbf{L L}}^{\mathrm{B} 2}
\end{array}\right]} \\
{\left[\mathbf{K}_{\mathbf{L C}}^{\mathrm{B}}\right]=\left[\begin{array}{cc}
\mathbf{K}_{\mathbf{L C}}^{\mathrm{B} 1} & \mathbf{0} \\
\mathbf{0} & \mathbf{K}_{\mathbf{L C}}^{\mathrm{B} 2}
\end{array}\right]} \\
{\left[\mathbf{K}_{\mathbf{C L}}^{\mathrm{B}}\right]=\left[\begin{array}{cc}
\mathbf{K}_{\mathbf{C} \mathbf{L}}^{\mathrm{B}} & \mathbf{0} \\
\mathbf{0} & \mathbf{K}_{\mathbf{C L}}^{\mathrm{B} 2}
\end{array}\right]} \\
{\left[\mathbf{K}_{\mathbf{C C}}^{\mathrm{B}}\right]=\left[\begin{array}{cc}
\mathbf{K}_{\mathbf{C C}}^{\mathrm{B}} & \mathbf{0} \\
\mathbf{0} & \mathbf{K}_{\mathbf{C} \mathbf{C}}^{\mathrm{B} 2}
\end{array}\right]} \\
\left\{\mathbf{u}_{\mathbf{L}}^{\mathrm{B}}\right\}=\left\{\begin{array}{c}
\mathbf{u}_{\mathbf{L}}^{\mathrm{B} 1} \\
\mathbf{u}_{\mathbf{L}}^{\mathrm{B} 2}
\end{array}\right\} \\
\left\{\mathbf{u}_{\mathbf{C}}^{\mathrm{B}}\right\}=\left\{\begin{array}{l}
\mathbf{u}_{\mathbf{C}}^{\mathrm{B} 1} \\
\mathbf{u}_{\mathbf{C}}^{\mathrm{B} 2}
\end{array}\right\} \\
\left\{\mathbf{p}_{\mathbf{L}}^{\mathrm{B}}\right\}=\left\{\begin{array}{l}
\mathbf{p}_{\mathbf{L}}^{\mathrm{B} 1} \\
\mathbf{p}_{\mathbf{L}}^{\mathrm{B} 2}
\end{array}\right\}
\end{gathered}
$$

e

$$
\left\{\mathbf{p}_{\mathbf{C}}^{\mathrm{B}}\right\}=\left\{\begin{array}{l}
\mathbf{p}_{\mathbf{C}}^{\mathrm{B} 1} \\
\mathbf{p}_{\mathbf{C}}^{\mathrm{B} 2}
\end{array}\right\}
$$

A obtenção da matriz $\mathbf{K}^{\mathbf{B N}}$ para um $N$ qualquer segue um raciocínio análogo ao empregado para dois blocos. As equações 5.30 e 5.31 escritas para os blocos 1 e 2 devem ser obtidas para todos os demais blocos. Desta forma, a equação do n-ésimo bloco fica:

$$
\left[\mathbf{K}^{\mathbf{B n}}\right]\left\{\mathbf{u}^{\mathbf{B n}}\right\}=\left\{\mathbf{p}^{\mathbf{B n}}\right\}
$$


$\mathrm{Ou}$

$$
\left[\begin{array}{ll}
\mathbf{K}_{\mathbf{L L}}^{\mathrm{Bn}} & \mathbf{K}_{\mathbf{L C}}^{\mathrm{Bn}} \\
\mathbf{K}_{\mathrm{CL}}^{\mathrm{Bn}} & \mathbf{K}_{\mathbf{C C}}^{\mathrm{Bn}}
\end{array}\right]\left\{\begin{array}{l}
\mathbf{u}_{\mathbf{L}}^{\mathrm{Bn}} \\
\mathbf{u}_{\mathbf{C}}^{\mathrm{Bn}}
\end{array}\right\}=\left\{\begin{array}{l}
\mathbf{p}_{\mathbf{L}}^{\mathrm{Bn}} \\
\mathbf{p}_{\mathbf{C}}^{\mathrm{Bn}}
\end{array}\right\}
$$

Considerando um número qualquer de blocos, o vetor de deslocamentos $\mathbf{u}^{\mathbf{B N}}$ indicado na relação 5.34 é:

$$
\left\{\mathbf{u}^{\mathrm{BN}}\right\}=\left\{\begin{array}{c}
\mathbf{u}_{\mathbf{L}}^{\mathrm{B} 1} \\
\mathbf{u}_{\mathbf{L}}^{\mathrm{B} 2} \\
\vdots \\
\mathbf{u}_{\mathbf{L}}^{\mathrm{Bn}} \\
\mathbf{u}_{\mathrm{C}}^{\mathrm{B} 1} \\
\mathbf{u}_{\mathrm{C}}^{\mathrm{B} 2} \\
\vdots \\
\mathbf{u}_{\mathrm{C}}^{\mathrm{Bn}}
\end{array}\right\}
$$

e o vetor de forças $\mathbf{p}^{\mathbf{B N}}$ se torna:

$$
\left\{p^{B N}\right\}=\left\{\begin{array}{c}
p_{\mathrm{L}}^{\mathrm{B1}} \\
\mathrm{p}_{\mathrm{L}}^{\mathrm{B2}} \\
\vdots \\
\mathrm{p}_{\mathrm{L}}^{\mathrm{Bn}} \\
\mathrm{p}_{\mathrm{C}}^{\mathrm{B1}} \\
\mathrm{p}_{\mathrm{C}}^{\mathrm{B} 2} \\
\vdots \\
\mathrm{p}_{\mathrm{C}}^{\mathrm{Bn}}
\end{array}\right\}
$$

A partir dos vetores 5.48 e 5.49 e analogamente à equação 5.36, obtém-se a equação:

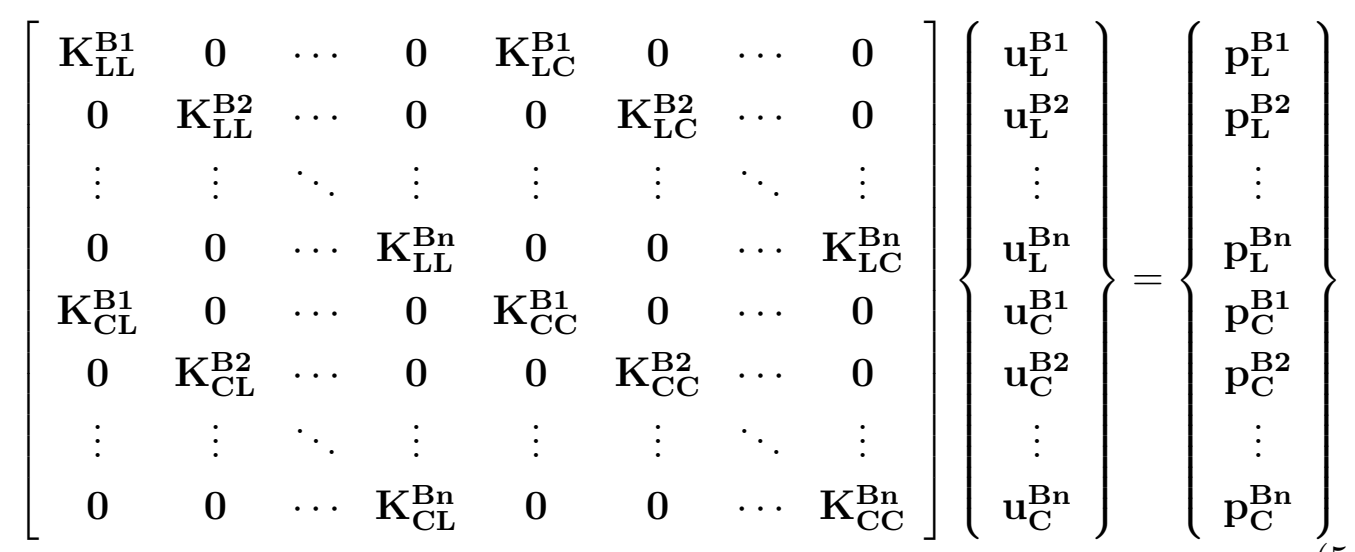

Assim como na equação 5.36, pode-se observar na equação 5.50 a independência entre as parcelas referentes a cada bloco. Isto ocorre porque não existem nós de contato entre os blocos. 
Finalizando, pode-se utilizar a equação 5.6 para um número qualquer de blocos. Para isto, devem ser feitas as seguintes associações:

$$
\begin{aligned}
& {\left[\mathbf{K}_{\mathbf{L L}}^{\mathrm{B}}\right]=\left[\begin{array}{cccc}
\mathbf{K}_{\mathbf{L L}}^{\mathrm{B} 1} & \mathbf{0} & \cdots & \mathbf{0} \\
\mathbf{0} & \mathbf{K}_{\mathbf{L L}}^{\mathrm{B2}} & \cdots & 0 \\
\vdots & \vdots & \ddots & \vdots \\
0 & 0 & \cdots & \mathbf{K}_{\mathbf{L L}}^{\mathrm{Bn}}
\end{array}\right]} \\
& {\left[\mathbf{K}_{\mathbf{L C}}^{\mathrm{B}}\right]=\left[\begin{array}{cccc}
\mathbf{K}_{\mathbf{L C}}^{\mathrm{B} 1} & 0 & \cdots & 0 \\
0 & \mathbf{K}_{\mathbf{L C}}^{\mathrm{B2}} & \cdots & 0 \\
\vdots & \vdots & \ddots & \vdots \\
0 & 0 & \cdots & \mathbf{K}_{\mathbf{L C}}^{\mathrm{Bn}}
\end{array}\right]} \\
& {\left[\mathbf{K}_{\mathbf{C L}}^{\mathrm{B}}\right]=\left[\begin{array}{cccc}
\mathbf{K}_{\mathbf{C L}}^{\mathrm{B} 1} & \mathbf{0} & \cdots & \mathbf{0} \\
\mathbf{0} & \mathbf{K}_{\mathbf{C L}}^{\mathrm{B} 2} & \cdots & 0 \\
\vdots & \vdots & \ddots & \vdots \\
0 & 0 & \cdots & \mathbf{K}_{\mathbf{C L}}^{\mathrm{Bn}}
\end{array}\right]} \\
& {\left[\mathbf{K}_{\mathbf{C C}}^{\mathrm{B}}\right]=\left[\begin{array}{cccc}
\mathbf{K}_{\mathbf{C C}}^{\mathrm{B} 1} & 0 & \cdots & 0 \\
0 & \mathbf{K}_{\mathbf{C C}}^{\mathrm{B} 2} & \cdots & 0 \\
\vdots & \vdots & \ddots & \vdots \\
0 & 0 & \cdots & \mathbf{K}_{\mathbf{C C}}^{\mathrm{Bn}}
\end{array}\right]} \\
& \left\{\mathbf{u}_{\mathbf{L}}^{\mathrm{B}}\right\}=\left\{\begin{array}{c}
\mathbf{u}_{\mathbf{L}}^{\mathrm{B} 1} \\
\mathbf{u}_{\mathbf{L}}^{\mathrm{B} 2} \\
\vdots \\
\mathbf{u}_{\mathbf{L}}^{\mathrm{Bn}}
\end{array}\right\} \\
& \left\{\mathbf{u}_{\mathrm{C}}^{\mathrm{B}}\right\}=\left\{\begin{array}{c}
\mathbf{u}_{\mathrm{C}}^{\mathrm{B} 1} \\
\mathbf{u}_{\mathrm{C}}^{\mathrm{B} 2} \\
\vdots \\
\mathbf{u}_{\mathrm{C}}^{\mathrm{Bn}}
\end{array}\right\} \\
& \left\{p_{\mathbf{L}}^{B}\right\}=\left\{\begin{array}{c}
p_{\mathbf{L}}^{\mathrm{B} 1} \\
\mathbf{p}_{\mathbf{L}}^{\mathrm{B} 2} \\
\vdots \\
\mathrm{p}_{\mathbf{L}}^{\mathrm{Bn}}
\end{array}\right\}
\end{aligned}
$$




$$
\left\{\mathbf{p}_{\mathbf{C}}^{\mathrm{B}}\right\}=\left\{\begin{array}{c}
\mathbf{p}_{\mathbf{C}}^{\mathrm{B} 1} \\
\mathbf{p}_{\mathbf{C}}^{\mathrm{B} 2} \\
\vdots \\
\mathbf{p}_{\mathbf{C}}^{\mathrm{Bn}}
\end{array}\right\}
$$

\subsection{Acoplamento entre o solo e o edifício}

Assim como foi feito para os blocos, o acoplamento entre as formulações do solo com o conjunto formado pelo edifício e a placa, ilustrado na figura 5.2, é baseado em condições de compatibilidade de deslocamentos e equilíbrio de forças nos nós de contato entre os dois meios. É utilizada como ponto de partida a equação 4.61. resultado do Método da Rigidez Sucessiva aplicado ao solo estratificado, e a relação 3.20, advinda da simulação do edifício pelo MEF. Estas equações podem ser escritas, respectivamente, como:

$$
\begin{aligned}
{\left[\mathbf{K}_{\text {MEC }}\right]\left\{\mathbf{u}_{\mathbf{M E C}}\right\} } & =\left\{\mathbf{p}_{\mathbf{M E C}}\right\} \\
{\left[\mathbf{K}_{\mathbf{M E F}}\right]\left\{\mathbf{u}_{\mathbf{M E F}}\right\} } & =\left\{\mathbf{f}_{\mathbf{M E F}}\right\}
\end{aligned}
$$

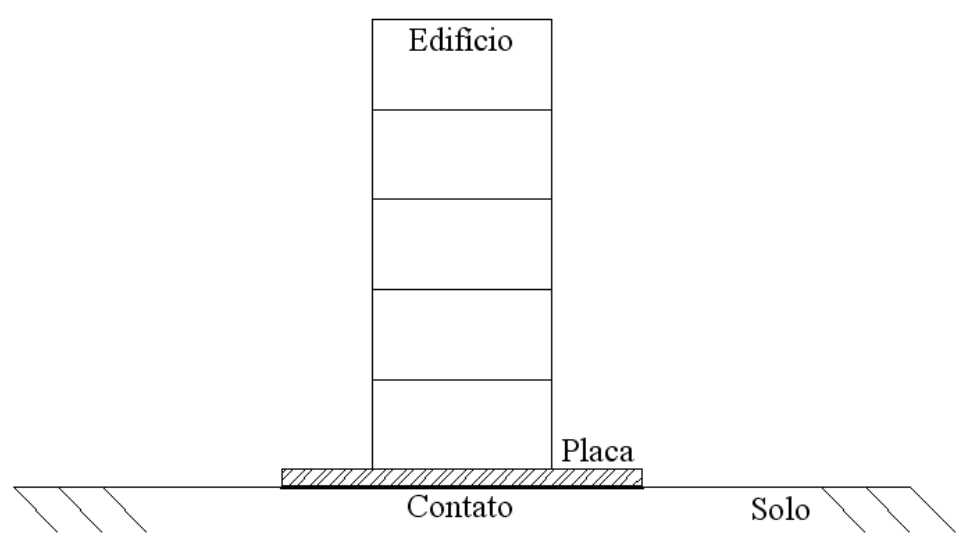

Figura 5.2: Interação do solo com o edifício

Antes de serem relacionadas, as matrizes e vetores das equações 5.59 e 5.60 são expandidos de forma a obter duas equações que levem em conta todos os nós da superfície do solo e do edifício. Como resultado tem-se as equações:

$$
\begin{array}{r}
{\left[\overline{\mathbf{K}}_{\text {MEC }}\right]\{\mathbf{u}\}=\left\{\overline{\mathbf{p}}_{\mathrm{MEC}}\right\}} \\
{\left[\overline{\mathbf{K}}_{\mathrm{MEF}}\right]\{\mathbf{u}\}=\left\{\overline{\mathbf{f}}_{\mathrm{MEF}}\right\}}
\end{array}
$$

O vetor u contém os deslocamentos nos nós de ambos os meios. Em sua montagem foram aplicadas condições de compatibilidade de deslocamentos nos nós do 
contato, possibilitando a obtenção de um único vetor. As matrizes $\overline{\mathbf{K}}_{\text {MEC }}$ e $\overline{\mathbf{K}}_{\text {MEF }}$ possuem trechos de zeros devido às expansões, bem como os vetores $\overline{\mathbf{p}}_{\mathbf{M E C}}$ e $\overline{\mathbf{f}}_{\text {MEF }}$.

Considerando o equilíbrio existente no contato entre o solo e o edifício, é possível relacionar as equações 5.61 e 5.62 considerando o vetor de forças $\overline{\mathbf{p}}_{\mathbf{M E C}}$ como cargas reativas no vetor $\overline{\mathbf{f}}_{\mathbf{M E F}}$. Entretanto, não é possível realizar esta operação subtraindo diretamente do vetor $\overline{\mathbf{f}}_{\text {MEF }}$ o vetor $\overline{\mathbf{p}}_{\text {MEC }}$ devido à incompatibilidade que existe entre as forças contidas nos vetores. Isto pode ser visualizado na figura 5.3. No MEC as forças são tratadas como cargas por unidade de área, enquanto que no MEF as forças são tratadas com cargas concentradas nos nós.
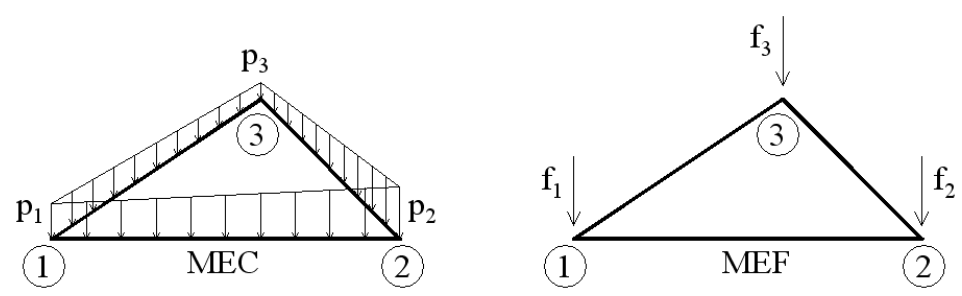

Figura 5.3: Incompatibilidade entre as cargas de superfície e as cargas nodais

Para compatibilizar os vetores, foram estudadas em ALMEIDA (2003a) duas possibilidades. A primeira é transformar o vetor $\overline{\mathbf{f}}_{\mathbf{M E F}}$ em um vetor de cargas de superfície equivalente, e a segunda é transformar o vetor $\overline{\mathbf{p}}_{\mathbf{M E C}}$ em um vetor de cargas nodais equivalente. O critério adotado para optar entre um ou outro procedimento foi o custo computacional envolvido nas transformações. Assim, optou-se por transformar o vetor $\overline{\mathbf{p}}_{\text {MEC }}$ em um vetor de cargas nodais equivalente por ser de menor custo computacional.

Como o procedimento a ser realizado é o mesmo para as três direções do sistema de coordenadas global $x_{1} x_{2} x_{3}$, por questão de praticidade, nas próximas deduções é tomada uma única direção.

Para transformar o vetor de cargas de superfície $\overline{\mathbf{p}}_{\text {MEC }}$ em um vetor de cargas nodais equivalente, parte-se do trabalho realizado por estas cargas de superfície. Este trabalho, analisado em um único elemento finito, pode ser representado pela expressão:

$$
T=\int_{A} p\left(\xi_{1}, \xi_{2}, \xi_{3}\right) w\left(\xi_{1}, \xi_{2}, \xi_{3}\right) d A
$$

em que $\xi_{1}$ e $\xi_{2}$ são coordenadas locais definidas no elemento e $\xi_{3}$ é função das outras duas. A função $p$ é uma aproximação das forças de superfície atuantes no elemento, e a função $w$ é uma aproximação do campo de deslocamentos do elemento. A área do elemento está indicada pela letra $A$.

Como na formulação do MEC apresentada no capítulo 2 é considerada uma aproximação linear de forças e deslocamentos, conforme ilustrado na figura 5.4, as aproximações de $w$ e $p$ são adotadas como lineares. Esta aproximação foi descrita 
no capítulo 2, e resultou nas funções:

$$
w\left(\xi_{1}, \xi_{2}, \xi_{3}\right)=w_{1} \xi_{1}+w_{2} \xi_{2}+w_{3} \xi_{3}
$$

e

$$
p\left(\xi_{1}, \xi_{2}, \xi_{3}\right)=p_{1} \xi_{1}+p_{2} \xi_{2}+p_{3} \xi_{3}
$$

com

$$
\xi_{3}=1-\xi_{1}-\xi_{2}
$$
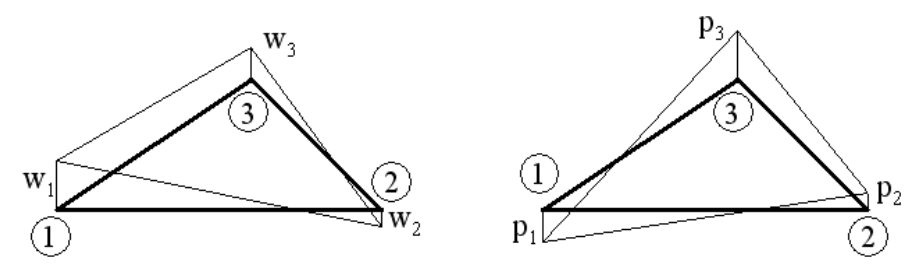

Figura 5.4: Aproximações lineares adotadas para $w$ e $p$

Substituindo as funções 5.64 e 5.65 na expressão 5.63 , obtém-se a expressão:

$$
T=\int_{A}\left(w_{1} \xi_{1}+w_{2} \xi_{2}+w_{3} \xi_{3}\right)\left(p_{1} \xi_{1}+p_{2} \xi_{2}+p_{3} \xi_{3}\right) d A
$$

A expressão final do trabalho realizado pelas forças de superfície é obtida desenvolvendo-se a expressão 5.67 e efetuando-se a integral. Neste procedimento, uma ferramenta útil é a seguinte fórmula:

$$
\int_{A} \xi_{1}^{\eta 1} \xi_{2}^{\eta 2} \xi_{3}^{\eta 3} d A=2 A \frac{\eta_{1} ! \eta_{2} ! \eta_{3} !}{\left(\eta_{1}+\eta_{2}+\eta_{3}+2\right) !}
$$

Depois de chegar à expressão final do trabalho realizado pelas forças de superfície, deve-se minimizar o funcional da energia potencial acumulada no elemento finito. Ao final das deduções, chega-se à igualdade:

$$
\left\{\begin{array}{l}
f_{1} \\
f_{2} \\
f_{3}
\end{array}\right\}=\frac{A}{12}\left[\begin{array}{lll}
2 & 1 & 1 \\
1 & 2 & 1 \\
1 & 1 & 2
\end{array}\right]\left\{\begin{array}{l}
p_{1} \\
p_{2} \\
p_{3}
\end{array}\right\}
$$

O vetor à direita da igualdade em 5.69 contém as cargas de superfície nos nós de um elemento qualquer da superfície do solo, segundo uma direção qualquer. O vetor à esquerda contém as cargas nodais equivalentes, obtido por meio da matriz 
de transformação à direita. Uma forma mais compacta da relação 5.69 é:

$$
\{\mathbf{f}\}=[\overline{\mathbf{Q}}]\{\mathbf{p}\}
$$

com

$$
[\overline{\mathbf{Q}}]=\frac{A}{12}\left[\begin{array}{lll}
2 & 1 & 1 \\
1 & 2 & 1 \\
1 & 1 & 2
\end{array}\right]
$$

Considerando a relação 5.70 aplicada a todos os elementos, obtém-se a igualdade:

$$
\{\mathbf{r}\}=[\mathbf{Q}]\left\{\overline{\mathbf{p}}_{\mathbf{M E C}}\right\}
$$

$\mathrm{O}$ vetor $\mathbf{r}$ contém as cargas nodais equivalentes às forças de superfície contidas no vetor $\overline{\mathbf{p}}_{\text {MEC }}$. O efeito do vetor $\mathbf{r}$ pode ser considerado na equação 5.62 caso ele seja encarado como um conjunto de forças reativas atuantes na base do edifício. Desta forma, chega-se à equação:

$$
\left[\mathbf{K}_{\mathrm{MEF}}\right]\{\mathbf{u}\}=\left\{\overline{\mathbf{f}}_{\mathrm{MEF}}\right\}-\{\mathbf{r}\}
$$

Aplicando a igualdade 5.72 na equação 5.73 , conclui-se que:

$$
\left[\overline{\mathbf{K}}_{\mathrm{MEF}}\right]\{\mathbf{u}\}=\left\{\overline{\mathbf{f}}_{\mathrm{MEF}}\right\}-[\mathbf{Q}]\left\{\overline{\mathbf{p}}_{\mathrm{MEC}}\right\}
$$

Finalmente, considera-se a igualdade dada pela equação 5.61 na equação 5.74 . Assim:

$$
\left[\overline{\mathbf{K}}_{\mathbf{M E F}}\right]\{\mathbf{u}\}=\left\{\overline{\mathbf{f}}_{\mathbf{M E F}}\right\}-[\mathbf{Q}]\left[\overline{\mathbf{K}}_{\mathrm{MEC}}\right]\{\mathbf{u}\}
$$

Para chegar ao sistema de equações final, basta desenvolver a equação 5.75. Desta forma:

$$
\left(\left[\overline{\mathbf{K}}_{\mathbf{M E F}}\right]+[\mathbf{Q}]\left[\overline{\mathbf{K}}_{\mathbf{M E C}}\right]\right)\{\mathbf{u}\}=\left\{\overline{\mathbf{f}}_{\mathrm{MEF}}\right\}
$$

Por fim:

$$
[\mathbf{K}]\{\mathbf{u}\}=\{\mathbf{f}\}
$$

A matriz $\mathbf{K}$ representa o acoplamento entre o MEC e o MEF, e contém a influência das camadas de solo e do edifício. O vetor u contém os deslocamentos dos nós da superfície livre do solo e do edifício, sendo todos incógnitos. O vetor f guarda as cargas externas aplicadas no edifício. A resolução do sistema de equações 5.77 permite a obtenção dos deslocamentos $\mathbf{u}$. 


\subsection{Acoplamento entre solo, blocos e edifício}

Quando o edifício é acoplado ao conjunto formado pelos blocos e o solo, surge um problema a ser contornado. O fato do edifício ter sido modelado pelo MEF e os blocos pelo MEC torna diferentes os graus de liberdade definidos na base dos pilares e nos pontos dos blocos. Conforme explicado no capítulo 3 os nós dos elementos reticulares e planos do edifício têm seis graus de liberdade, sendo três deslocamentos e três rotações. Já os nós dos blocos, que são modelados pelo MEC conforme descrito no capítulo 2, só possuem três graus de liberdade, que são os deslocamentos. Isto está ilustrado na figura 5.5 .

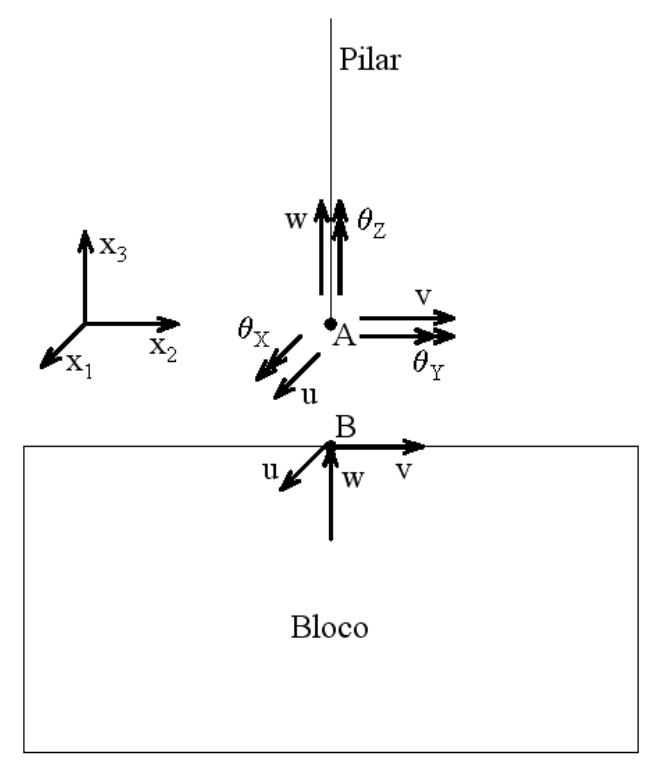

Figura 5.5: Graus de liberdade do MEF e do MEC

Na figura 5.5, o ponto $A$ representa a extremidade do pilar e o ponto $B$ pertence ao bloco. Os deslocamentos estão indicados por $u, v$ e $w$, enquanto as rotações estão indicadas por $\theta_{X}, \theta_{Y}$ e $\theta_{Z}$.

Devido a essa diferença existente entre os graus de liberdade, caso os pilares fossem diretamente conectados aos blocos, as três rotações ficariam livres. Deste modo existiria sempre uma rótula na base dos pilares, algo que tornaria o programa limitado. Outra opção seria restringir as três rotações na base de todos os pilares, mas então as ações advindas do edifício não seriam totalmente transferidas para os blocos. Isto entraria em contradição com um dos propósitos deste trabalho, que é analisar o comportamento da estrutura em conjunto com o solo.

A solução adotada neste trabalho foi definir uma casca flexível conectada ao topo de cada bloco, conforme ilustrado na figura 5.6. Esta casca é modelada com elementos finitos triangulares DKT/FF, sendo adotados módulo de elasticidade e coeficiente de Poisson iguais aos do bloco. A espessura da casca é assumida igual a um quarto da altura do bloco. 


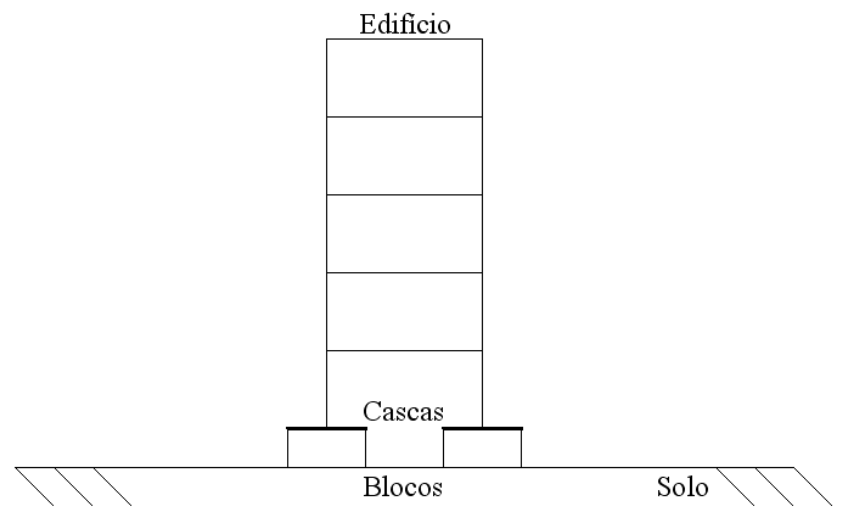

Figura 5.6: Conjunto formado pelo solo, blocos e edifício

Esta opção pode ser considerada mais vantajosa que conectar a base dos pilares diretamente ao bloco, pois é possível conectar os seis graus de liberdade da extremidade do pilar à casca. Apesar de que em cada ponto da casca somente os três deslocamentos são conectados ao bloco, o fato de vários pontos estarem conectados permite que as rotações ocorridas na base do pilar sejam indiretamente transmitidas ao bloco por meio de binários de força. Isto pode ser visualizado na figura 5.7 .

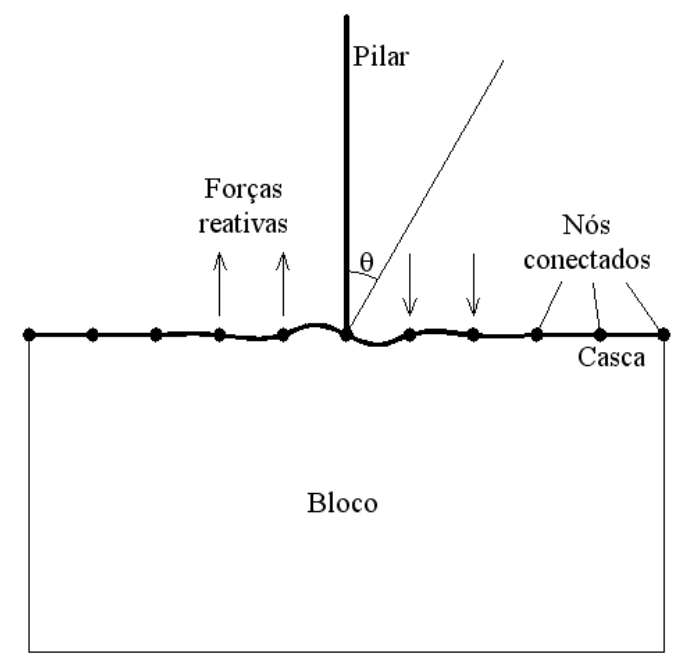

Figura 5.7: Casca flexível conectada ao topo do bloco

A figura 5.7 ilustra uma rotação $\theta$ do pilar sendo transmitida ao bloco por meio da casca. É mostrado o que ocorre com uma das rotações, sendo o raciocínio para as outras duas análogo.

Outra vantagem de utilizar uma casca intermediando a conecção entre o bloco e o edifício é que a teoria utilizada neste acoplamento é a mesma aplicada na seção 5.4, portanto não precisa ser deduzida novamente. 


\subsection{Considerações finais}

Foi apresentada, neste capítulo, a formulação empregada no acoplamento entre solo e estrutura. Dois tipos de estrutura foram analisados interagindo com o solo, sendo o primeiro blocos tridimensionais modelados pelo MEC e o segundo um edifício tridimensional modelado pelo MEF. Também foi descrito o acoplamento de um edifício apoiado sobre blocos, formando o conjunto solo/blocos/edifício.

Na parte inicial do capítulo foram apresentadas as deduções relativas à interação do solo com o bloco, ambos modelados pelo MEC. Por ser um problema de subregiões do MEC, a interação destes dois subsistemas foi resolvida aplicando condições de compatibilidade de deslocamentos e equilíbrio de forças nos nós de contato entre os dois meios. Como resultado, obteve-se um sistema de equações cuja solução são os deslocamentos nos nós das superfícies do solo e do bloco que não pertencem ao contato. Em seguida, a formulação foi generalizada para um número qualquer de blocos apoiados sobre o solo.

Para possibilitar a análise da interação do solo com o edifício de forma acoplada, foi necessário obter um único sistema de equações para representar os dois meios. Sendo o solo simulado pelo MEC e o édifício pelo MEF, ocorre uma incompatibilidade nos vetores de força advindos de cada técnica numérica. A solução adotada foi representar o vetor de forças de superfície originado no MEC por um vetor de cargas nodais equivalentes.

O acoplamento do edifício com o conjunto formado pelo solo e os blocos foi feito utilizando-se a mesma metodologia empregada no acoplamento do edifício com o solo sem blocos. Para uma melhor transferência das ações do edifício para os blocos, empregou-se cascas flexíveis para intermediar o contato das bases dos pilares com os topos dos blocos. 


\section{Capítulo 6}

\section{Exemplos}

\subsection{Introdução}

Neste capítulo são apresentados exemplos, demonstrando assim a funcionalidade do programa utilizado e das extensões densenvolvidas neste trabalho.

\subsection{Bloco sem estacas}

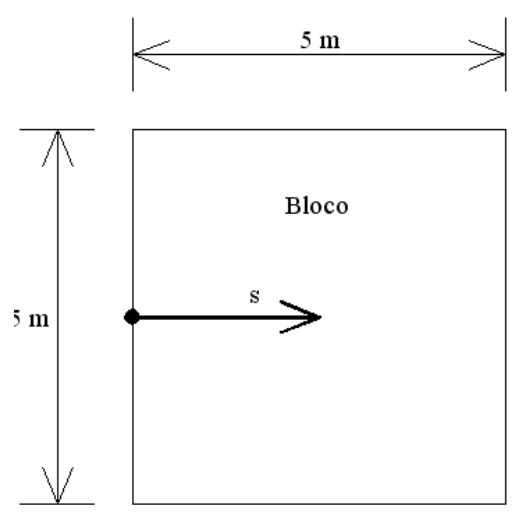

Figura 6.1: Vista em planta

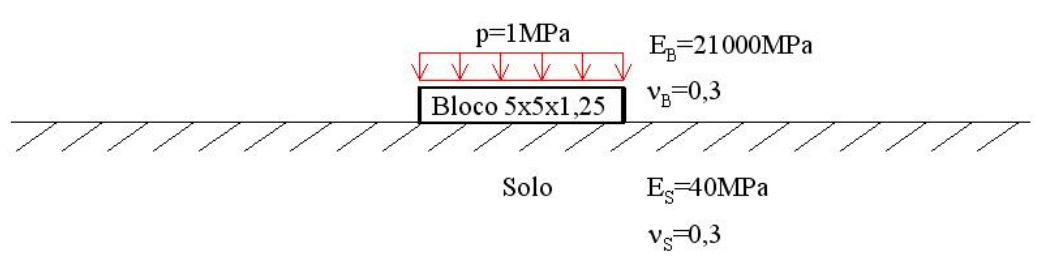

Figura 6.2: Vista em perfil

Neste exemplo é analisado um bloco sem estacas apoiado no solo, conforme ilustrado nas figuras 6.1 e 6.2. No final do exemplo, é estudada a influência de uma superfície de deslocamento nulo a uma distância prescrita. 
As dimensões do bloco são de 5 por 5 metros em planta e 1,25 metros de altura. O módulo de elasticidade adotado para o bloco, compatível ao do concreto, é de $21000000 \mathrm{kN} / \mathrm{m}^{2}$ ou 21000MPa. O coeficiente de Poisson adotado para o bloco é de 0,3. O solo, assumido inicialmente como um semi-espaço infinito, tem módulo de elasticidade igual a $40000 k N / m^{2}$ ou $40 \mathrm{MPa}$ e coeficiente de Poisson igual a 0,3 . A carga externa, aplicada no topo do bloco de forma uniformemente distribuída, é de $1000 \mathrm{kN} / \mathrm{m}^{2}$ ou $1 \mathrm{MPa}$. O eixo $s$, apresentado na figura 6.1, tem origem no ponto médio da lateral da base do bloco.

Em todos os valores apresentados, as unidades estão em metros e kN. Como primeiro resultado obtido pelo programa desenvolvido neste trabalho, são mostrados os deslocamentos verticais ocorridos na superfície do solo. A figura 6.3 ilustra os valores em metros obtidos pelo programa, considerando uma vista em planta. Optouse por mostrar somente a região próxima do bloco porque, a distâncias superiores a 10 metros do centro do bloco, os deslocamentos resultam praticamente nulos.

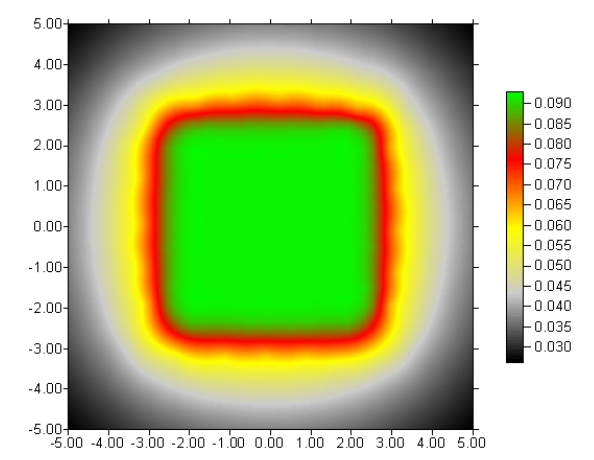

Figura 6.3: Vista em planta dos deslocamentos verticais

Conforme pode ser observado na figura 6.3, os deslocamentos ocorridos no contato são simétricos e praticamente constantes. A simetria ocorre devido ao carregamento externo, aplicado de forma simétrica no topo do bloco. A pequena variação nos deslocamentos pode ser explicada pela alta rigidez do bloco quando comparado com o solo, sendo a relação entre os dois de 525. A área onde ocorreram os maiores deslocamentos, reconhecida pela cor verde, corresponde ao contato entre o bloco e o solo. Este é um comportamento esperado, uma vez que todo o carregamento do sistema está aplicado no topo do bloco.

O primeiro modelo escolhido para comparar resultados de deslocamento foi gerado pelo programa Ansys5.4, com o elemento finito tridimensional solid45. A escolha deste programa pode ser considerada adequada, pois nele é possível simular tanto o bloco como o solo empregando o MEF tridimensional. Isto é compatível com análise feita neste trabalho, que emprega o MEC tridimensional.

Os deslocamentos calculados pelo Ansys5.4 resultaram em uma figura praticamente equivalente à figura 6.3. Para comparar os modelos, tomou-se então o valor 
do deslocamento no centro do bloco. O deslocamento no centro do bloco calculado pelo programa desenvolvido neste trabalho é de aproximadamente 9,26 centímetros, enquanto o Ansys5.4 obteve 8,84 centímetros, implicando em uma diferença de aproximadamente $4,8 \%$.

O segundo modelo empregado para comparar valores de deslocamento foi a formulação desenvolvida em ALMEIDA (2003a). A única diferença entre as formulações empregadas neste trabalho e no trabalho de ALMEIDA (2003a) na resolução deste exemplo é que, em ALMEIDA (2003a), o bloco é modelado pelo MEF com elementos finitos de casca planos. Neste trabalho, o bloco é modelado pelo MEC tridimensional.

A solução obtida para os deslocamentos segundo a formulação de ALMEIDA (2003a), da mesma forma que os valores do programa Ansys, resultaram em uma figura praticamente equivalente à figura 6.3. Tomou-se novamente então o valor do deslocamento no centro do bloco para comparar os modelos. Este valor, calculado pela formulação de ALMEIDA (2003a), é de 9,39 centímetros, enquanto o deslocamento obtido do programa desenvolvido neste trabalho é de 9,26 centímetros, implicando em uma diferença de $1,4 \%$.

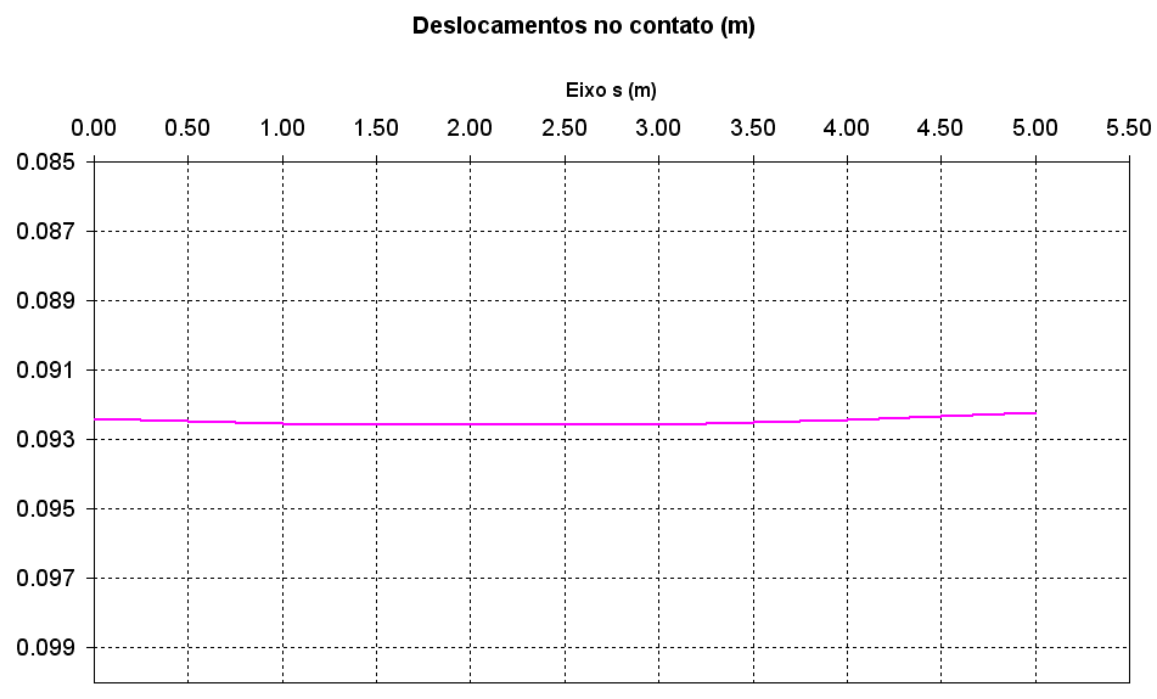

Figura 6.4: Deslocamentos no contato

Para uma melhor visualização dos deslocamentos, organizou-se no gráfico da figura 6.4 os deslocamentos ao longo do eixo $s$ ilustrado na figura 6.1. Neste gráfico pode ser observada boa simetria nos resultados, sendo a diferença de deslocamento entre as bordas de $0,2 \%$.

Um outro dado importante a ser apresentado é o das tensões de contato que ocorrem entre o solo e o bloco. Os valores calculados pelo programa desenvolvido neste trabalho são apresentados na figura 6.5, com unidades em metro e $k N$. Nesta figura está ilustrada a área de contato entre o bloco e o solo, de 5 por 5 metros, 


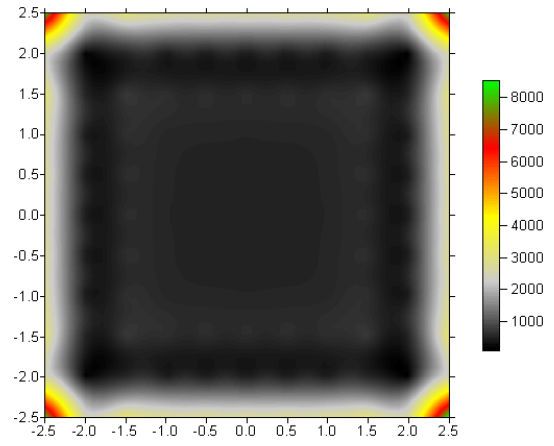

Figura 6.5: Tensões de contato

vista em planta. As tensões nas áreas centrais do contato, identificadas pelas cores escuras, são menores e com pouca variação. Esta distribuição homogênea pode ser explicada pelo carregamento aplicado de forma uniformemente distribuída no topo do bloco. As tensões mais elevadas, identificadas na figura 6.5 pela cor verde, se estabelecem nas regiões próximas às bordas do bloco. Este comportamento pode ser atribuído ao alto módulo de elasticidade do bloco em relação ao solo, impedindo o bloco de moldar-se ao maciço.

Para comparar os valores de tensões escolheu-se a formulação de ALMEIDA (2003a), devido às semelhanças com o modelo empregado neste trabalho. A solução das tensões de contato obtida pela formulação de ALMEIDA (2003a) resulta em uma figura praticamente igual à figura 6.5. Assim, para uma melhor comparação, foi feito o gráfico da figura 6.6. Todos os valores estão em metros e $k N$.

Tensões no contato $\left(\mathrm{KN} / \mathrm{m}^{2}\right)$

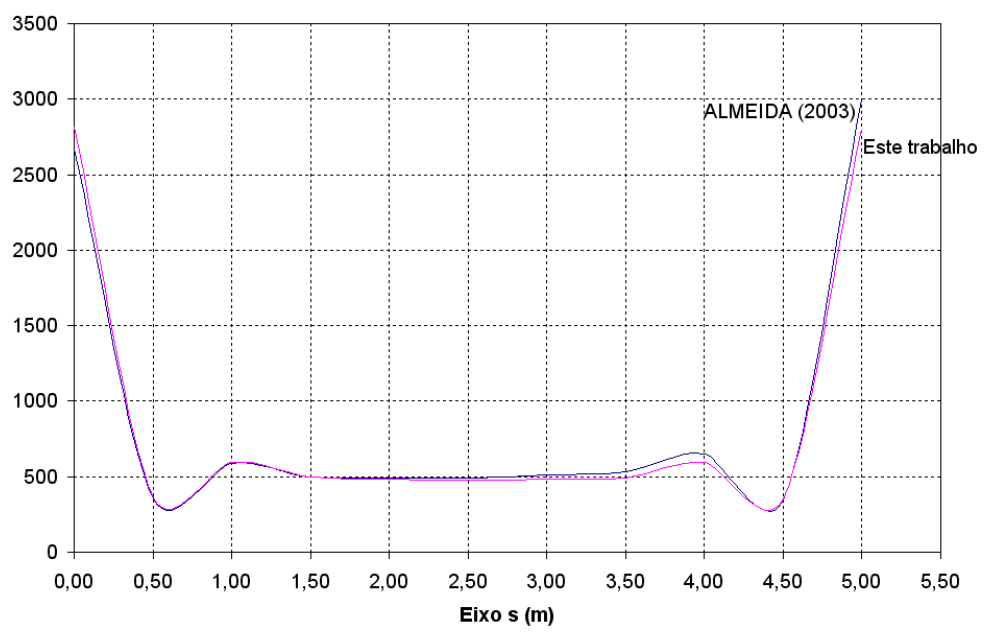

Figura 6.6: Tensões de contato

Neste gráfico, feito para pontos ao longo do eixo $s$ ilustrado na figura 6.1. pode-se perceber que tensões determinadas por cada modelo são muito próximas. Percebese de forma mais clara sua variação ao longo do contato, sendo quase constante na área central e atingindo valores elevados nas bordas. Pode ser observada também 
uma maior simetria no gráfico deste trabalho quando comparado aos valores de ALMEIDA (2003a).

É muito comum, em projetos reais de engenharia, a existência de uma superfície de deslocamentos nulos a uma certa distância da superfície. Assim sendo, é interessante estudar como a presença desta superfície influi na estrutura em estudo. Para avaliar-se esta influência, este exemplo será avaliado novamente prescrevendo uma superfície de deslocamentos nulos a uma profundidade de 10 metros. Isto pode ser visualizado na figura 6.7 .

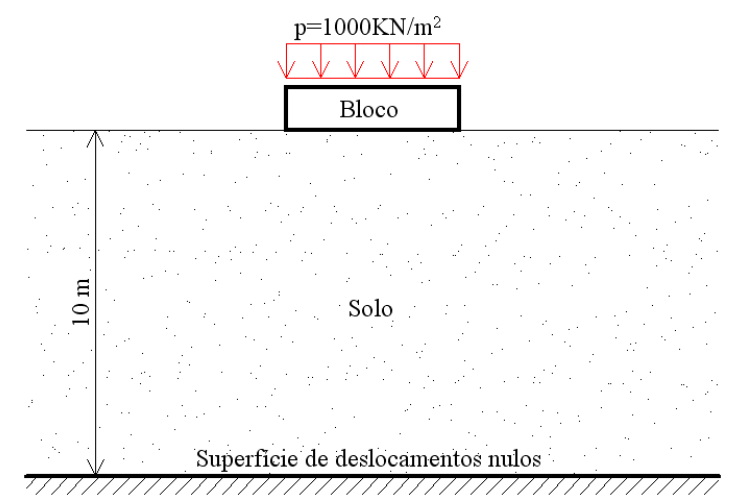

Figura 6.7: Superfície de deslocamentos nulos

As dimensões do bloco, propriedades físicas dos materiais e carregamento aplicado são os mesmos, sendo a única alteração a consideração da superfície de deslocamentos nulos. Com esta alteração, ocorreu uma sensível redução nos deslocamentos verticais ocorridos na superfície do solo, conforme pode ser visualizado na figura 6.8 quando comparada à figura 6.3. Os valores estão em metros.

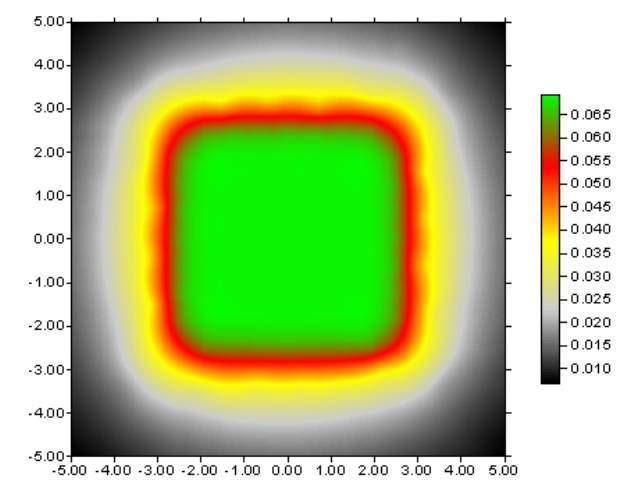

Figura 6.8: Deslocamentos verticais em planta considerando a superfície de deslocamentos nulos

Conforme pode ser observado na figura 6.8, os deslocamentos têm boa simetria. Seu comportamento é muito semelhante ao observado na figura 6.3 , sendo a única diferença relevante os valores atingidos em cada caso. O valor máximo de deslocamento caiu de 9,26 centímetros para 6,98 centímetros, valor 24,6\% inferior. Para 
uma melhor visualização desta diferença ao longo da base do bloco, foi feito o gráfico da figura 6.9 .

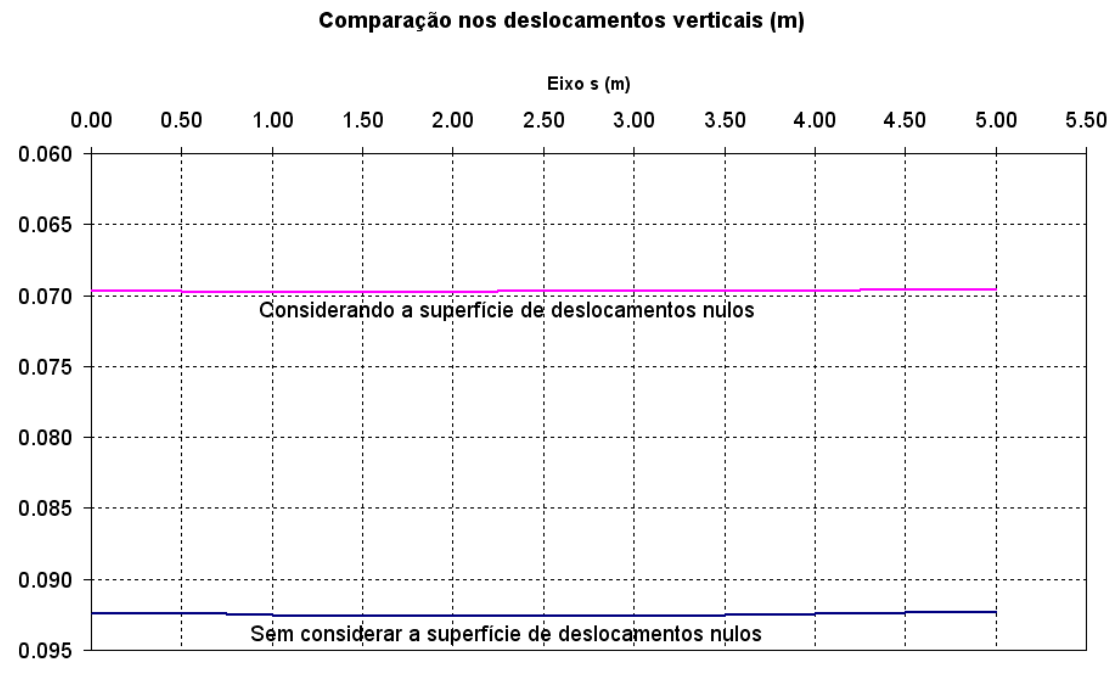

Figura 6.9: Comparação dos deslocamentos no contato

Neste gráfico estão representados os deslocamentos verticais em metros que ocorrem ao longo do eixo $s$, representado na figura 6.1. É possível observar a curvatura semelhante que ocorre nos dois casos, apesar da grande diferença nos valores.

A relevância desta diferença nos deslocamentos verticais pode ser melhor evidenciada imaginando que estes dois blocos analisados estejam servindo de apoio para uma edificação qualquer.

Simulando este caso com o Ansys5.4, obtém-se um deslocamento no centro do bloco de 6,81 centímetros. Isto resulta em uma diferença de $2,5 \%$ em relação ao valor de 6,98 centímetros encontrado utilizando o programa desenvolvido neste trabalho, indicando uma boa concordância entre os modelos.

As diferenças observadas nas tensões de contato não são tão significativas quanto as dos deslocamentos verticais. Os valores são tão próximos que nem é possível distingui-los com clareza, podendo-se concluir que a superfície de deslocamentos nulos não influi neste resultado.

Pelos resultados obtidos neste trabalho, quando comparados às demais formulações, pode-se concluir que o programa desenvolvido está coerente.

\subsection{Bloco sobre uma estaca}

O objetivo deste exemplo é estudar os efeitos decorrentes de uma estaca conectada à base de um bloco. A geometria do problema está ilustrada nas figuras 6.10 e 6.11. As dimensões do bloco são de 1 por 1 metro em planta e 1 metro de altura. A estaca, que é quadrada, tem 0,444 metro de diagonal e 4 metros de comprimento. 


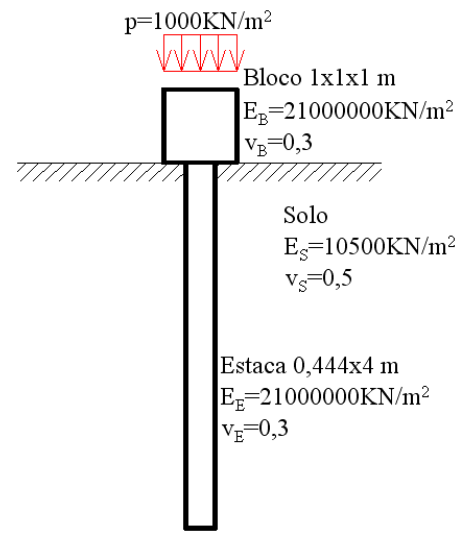

Figura 6.10: Vista em perfil

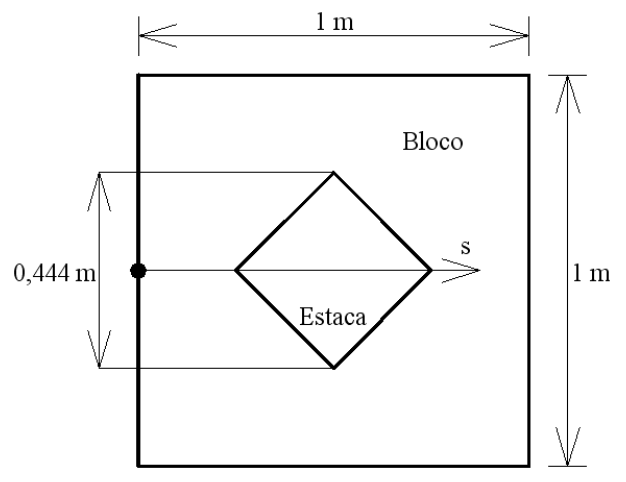

Figura 6.11: Vista em planta

O módulo de elasticidade adotado para o bloco e a estaca é de $21000000 k N / m^{2}$, e o coeficiente de Poisson para ambos é de 0,3 . O solo, assumido como um semi-espaço infinito, tem módulo de elasticidade igual a $10500 \mathrm{kN} / \mathrm{m}^{2}$ e coeficiente de Poisson igual a 0,5. A carga externa, aplicada verticalmente no topo do bloco de forma uniformemente distribuída, é de $1000 \mathrm{kN} / \mathrm{m}^{2}$. O eixo $s$, apresentado na figura 6.11 . tem origem no ponto médio da lateral da base do bloco.

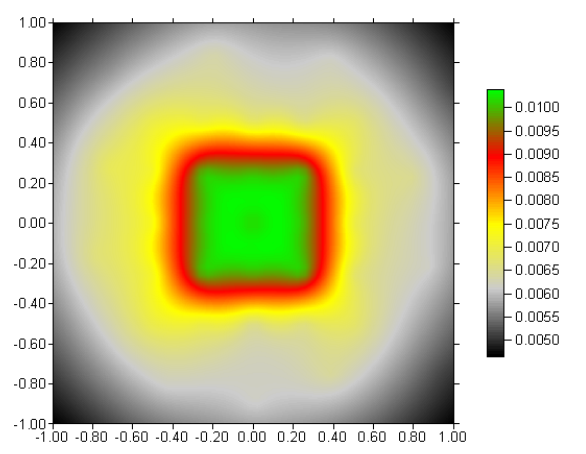

Figura 6.12: Vista em planta dos deslocamentos verticais

Os deslocamentos verticais calculados na superfície livre do solo pelo programa desenvolvido neste trabalho estão ilustrados, em metros, na figura 6.12. É possível observar pequenas assimetrias, as quais podem ser atribuídas à malha de elementos 
de contorno empregada na resolução do exemplo. Apesar da malha do bloco ser simétrica, a malha empregada para simular a superfície do solo, gerada no programa Ansys, resultou assimétrica devido à complexa geometria do problema. Para uma melhor análise do efeito causado nos deslocamentos pela presença da estaca, foi feito o gráfico da figura 6.13 .

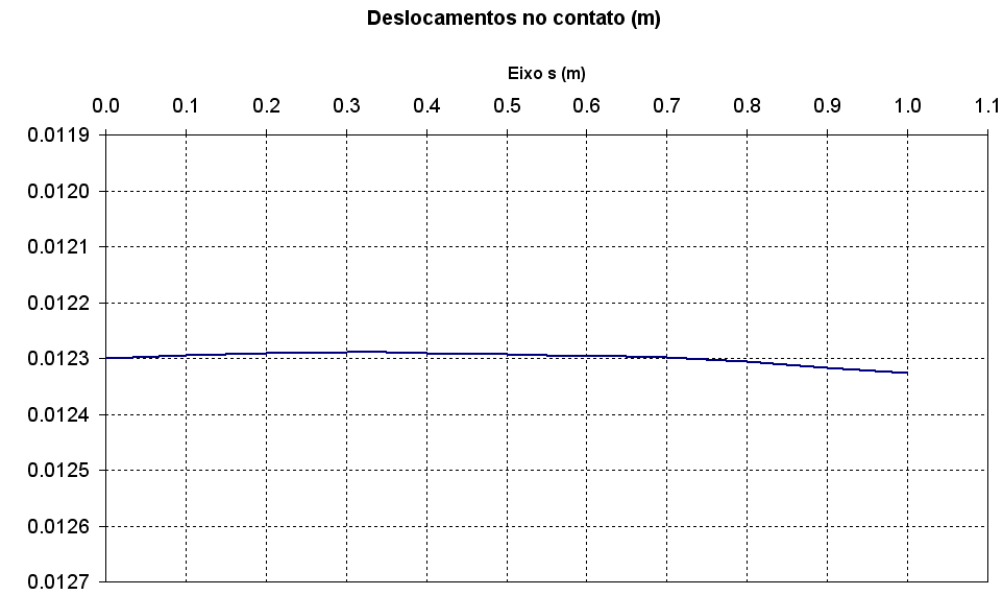

Figura 6.13: Deslocamentos no contato

Neste gráfico constam os valores do deslocamento vertical nos nós pertencentes ao eixo $s$, mostrado na figura 6.11. Comparando este gráfico com o gráfico da figura 6.4. no qual estão apresentados os deslocamentos em um bloco sem estacas, é possível perceber que a curvatura é invertida pela presença da estaca. A assimetria observada nos resultados pode ser desprezada, pois representa uma diferença de apenas $0,15 \%$ nos deslocamentos das bordas do bloco.

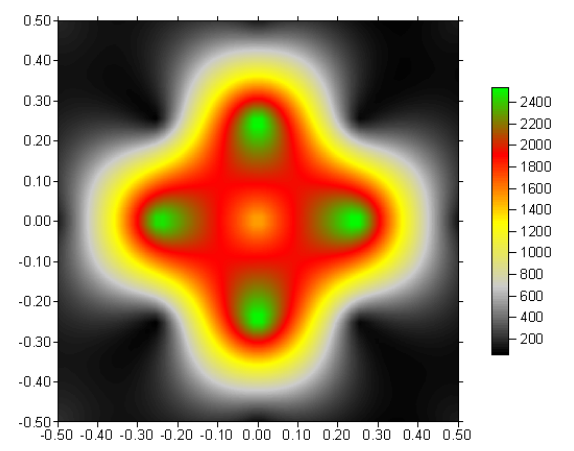

Figura 6.14: Vista em planta das tensões de contato

As tensões de contato calculadas pelo programa desenvolvido neste trabalho estão apresentadas, em $k N / m^{2}$, na figura 6.14. Conforme esperado, o valores mais elevados, indicados pela cor verde, ocorrem na parcela do contato onde está a estaca. Isto demonstra que a estaca absorve grande parte das cargas aplicadas no bloco, sendo o contato com o solo menos solicitado. Isto pode ser observado mais 
claramente no gráfico da figura 6.15. É possível notar que houve boa simetria neste resultado, e que as bordas da estaca absorvem mais carga que seu centro.

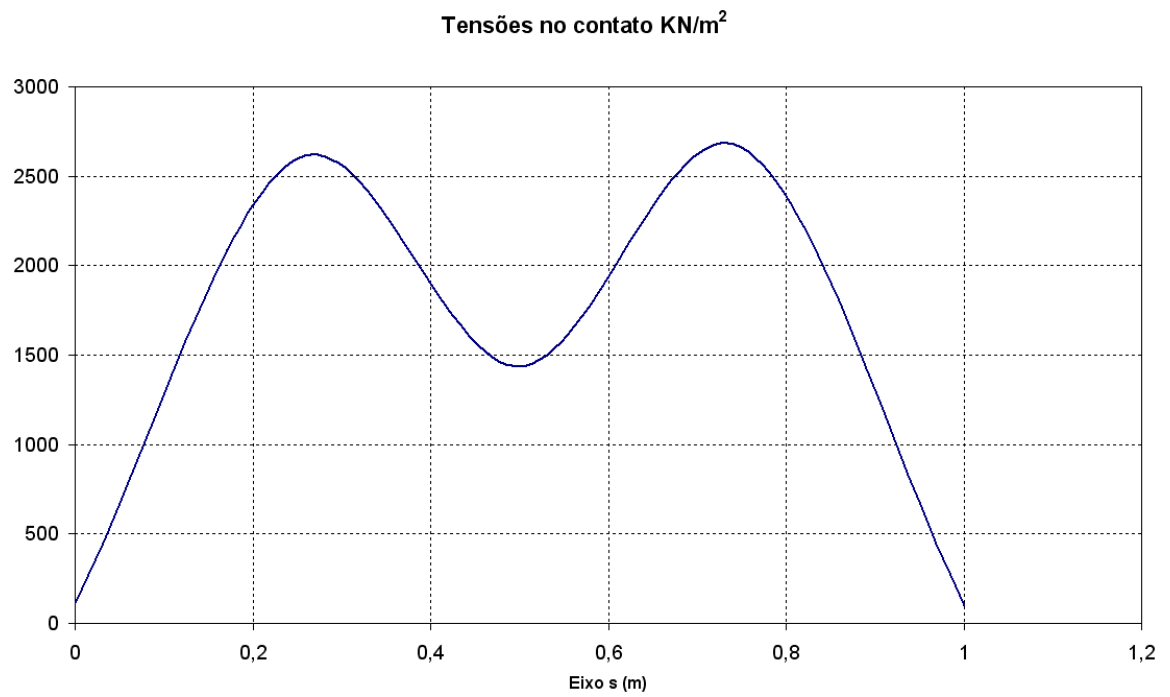

Figura 6.15: Tensões no contato

Para comprovar a consistência do programa serão comparados resultados com os publicados em BUTTERFIELD \& BANERJEE (1971), onde é analisada uma estaca conectada a uma lâmina rígida. Para simular esta lâmina empregou-se o bloco descrito no início deste item, pois este pode ser considerado equivalente à lâmina rígida, devido à sua espessura em conjunto com seu alto módulo de elasticidade em relação ao solo.

Em BUTTERFIELD \& BANERJEE (1971) são definidos alguns termos, os quais são explicados a seguir. O primeiro, denominado $\lambda$, é dado pela expressão:

$$
\lambda=\frac{E_{E}}{G_{S}}
$$

Na expressão 6.1. $E_{E}$ é o módulo de elasticidade longitudinal da estaca e $G_{S}$ é o módulo de elasticidade transversal do solo. O valor de $G_{S}$ é dado pela seguinte expressão:

$$
G_{S}=\frac{E_{S}}{2\left(1+\nu_{S}\right)}
$$

Como $E_{S}$ e $\nu_{S}$ são conhecidos, é possível substitui-los na expressão 6.2. Assim:

$$
G_{S}=\frac{10500}{2(1+0,5)}=3500 k N / m^{2}
$$

Substituindo os valores de $G_{S}$ e $E_{E}$ em 6.1, chega-se ao seguinte valor de $\lambda$ :

$$
\lambda=\frac{21000000}{3500}=6000
$$


Outras variáveis a serem definidas são o comprimento em planta do bloco:

$$
B=1 m
$$

a carga total $P$ aplicada ao sistema, igual à carga $p$ uniformemente distribuída vezes a área do topo do bloco:

$$
P=1000 \cdot 1 \cdot 1=1000 k N
$$

e o diâmetro $D$ da estaca. Em BUTTERFIELD \& BANERJEE (1971) o diâmetro $D$ é função do comprimento $B$ bloco, segundo a seguinte expressão:

$$
D=\frac{B}{2,5}
$$

Substituindo-se o valor de $B$ em 6.3, obtém-se o seguinte diâmetro de estaca:

$$
D=\frac{1}{2,5}=0,4 m
$$

Para compensar o fato de que a estaca considerada em BUTTERFIELD \& BANERJEE (1971) é de seção circular e a estaca considerada no programa desenvolvido neste trabalho é de seção quadrada escolheu-se, para a estaca quadrada, uma diagonal tal que seu perímetro seja igual ao de uma estaca circular de diâmetro igual a 0,4 metro. O perímetro $P_{c}$ de uma estaca circular de diâmetro igual a 0,4 metro pode ser calculado da seguinte forma:

$$
P_{c}=\pi D=0,4 \pi
$$

O perímetro $P_{q}$ de uma estaca quadrada de diagonal $D_{q}$ é:

$$
P_{q}=4 \frac{D_{q} \sqrt{2}}{2}=2 D_{q} \sqrt{2}
$$

Igualando $P_{c}$ a $P_{q}$, torna-se possível determinar a diagonal $D_{q}$ da estaca quadrada. Assim:

$$
\begin{gathered}
2 D_{q} \sqrt{2}=0,4 \pi \\
D_{q}=\frac{0,4 \pi}{2 \sqrt{2}} \approx 0,444 \mathrm{~m}
\end{gathered}
$$

Portanto, uma estaca circular de diâmetro igual a 0,4 metro pode ser aproximada por uma estaca quadrada de diagonal igual a 0,444 metro.

No estudo que é feito em BUTTERFIELD \& BANERJEE (1971), varia-se o comprimento da estaca e avalia-se este efeito no deslocamento ocorrido no topo da 
estaca. Com os resultados, monta-se um gráfico no qual as abcissas x correspondem ao cociente entre o comprimento $L$ da estaca e seu diâmetro $D$. Ou seja:

$$
x=\frac{L}{D}
$$

Os valores no eixo das coordenadas são calculados por meio da expressão:

$$
y=\frac{P}{G W D}
$$

Na expressão 6.5, $P$ é o valor da carga total aplicada ao sistema, $G$ é o módulo de elasticidade transversal $G_{S}$ do solo, $W$ é o deslocamento calculado no topo da estaca e $D$ é o diâmetro da estaca. Assim, variando o comprimento da estaca e calculando o valor de x e y pelas expressões 6.4 e 6.5, determinou-se o gráfico da figura 6.16.

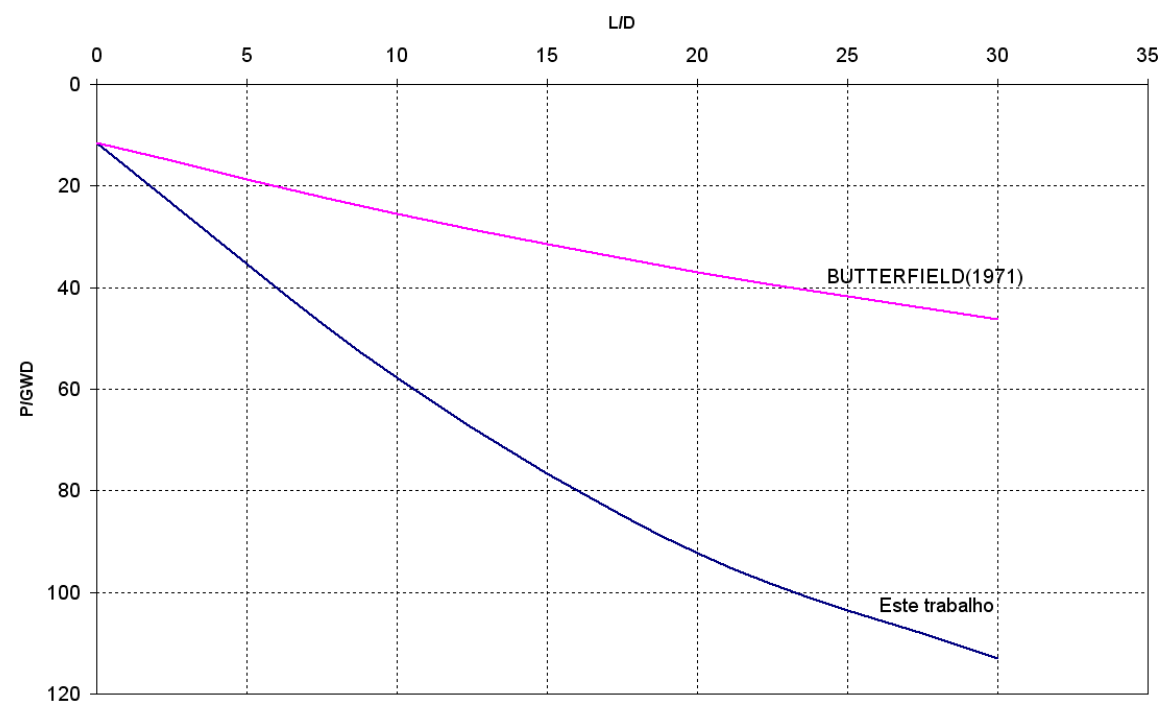

Figura 6.16: Deslocamento no topo da estaca

Conforme pode ser observado no gráfico desta figura, os valores coincidem no primeiro ponto da curva mas divergem com o aumento do comprimento da estaca. Como os valores de $y$, expressão 6.5, determinados pelo programa desenvolvido neste trabalho são maiores que os obtidos por BUTTERFIELD \& BANERJEE (1971), conclui-se que os deslocamentos obtidos por BUTTERFIELD \& BANERJEE (1971) resultaram maiores.

A diferença observada nas curvas pode ser atribuída às diversas diferenças existentes entre os modelos empregados neste trabalho e em BUTTERFIELD \& BANERJEE (1971). Algumas que podem ser citadas são a seção transversal considerada, a flexibilidade do bloco, a dimensão de cada modelo e a carga aplicada. Apesar do carregamento resultante ser o mesmo, no caso do bloco tridimensional este é aplicado em seu topo, enquanto que em BUTTERFIELD \& BANERJEE (1971) a carga é aplicada diretamente no topo da estaca. 
Foram analisados também outros exemplos envolvendo blocos sobre estacas. Foram também comparados com BUTTERFIELD \& BANERJEE (1971) os casos de um bloco sobre duas estacas e de um bloco sobre quatro estacas, sendo o gráfico resultante semelhante ao da figura 6.16. Como a conclusão é a mesma, estes exemplos não serão exibidos aqui.

\subsection{Interação entre dois blocos}

Neste exemplo são analisados dois blocos idênticos ao do item 6.2, para estudar a influência que ocorre entre eles. Assim sendo, as dimensões de cada bloco são de 5 por 5 metros em planta e 1,25 metros de altura. O módulo de elasticidade adotado para os blocos é de $21000000 \mathrm{kN} / \mathrm{m}^{2}$ e o coeficiente de Poisson de 0,3. O solo, assumido como um semi-espaço infinito, tem módulo de elasticidade igual a $40000 \mathrm{kN} / \mathrm{m}^{2}$ e coeficiente de Poisson igual a 0,3. A carga externa, aplicada verticalmente no topo de cada bloco de forma uniformemente distribuída, é de $1000 \mathrm{kN} / \mathrm{m}^{2}$. Estes blocos estão ilustrados nas figuras 6.17 e 6.18 .

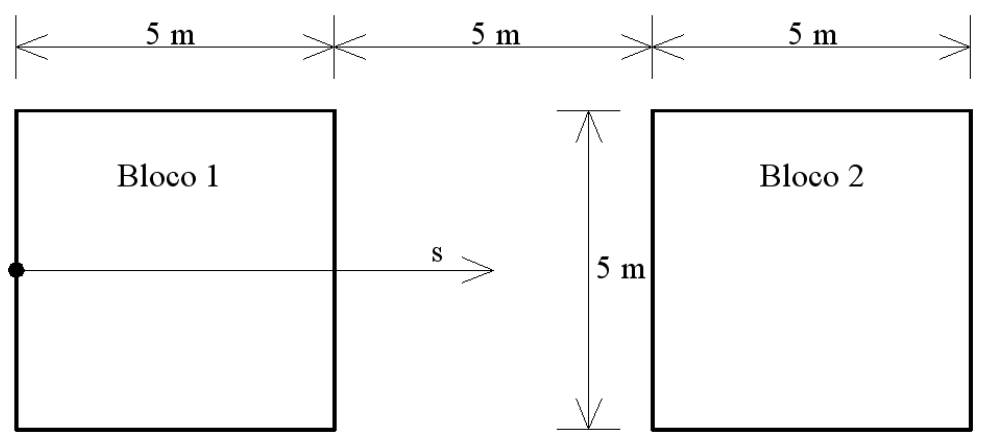

Figura 6.17: Vista em planta

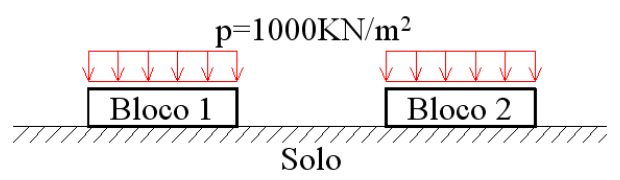

Figura 6.18: Vista em perfil

A distância adotada entre os blocos foi de 5 metros, conforme ilustrado na figura 6.17. O eixo $s$, com origem na face esquerda do bloco 1 , é utilizado como referência em alguns resultados. As unidades de todos os valores apresentados então em $k N$ e metro.

Na figura 6.19 estão ilustrados, em planta, os deslocamentos em metros ocorridos na superfície livre do solo na direção vertical. É possível observar que os maiores deslocamentos, indicados pela cor verde, ocorreram no contato entre os blocos e o solo. O valor máximo calculado foi de aproximadamente 9,88 centímetros, o que 


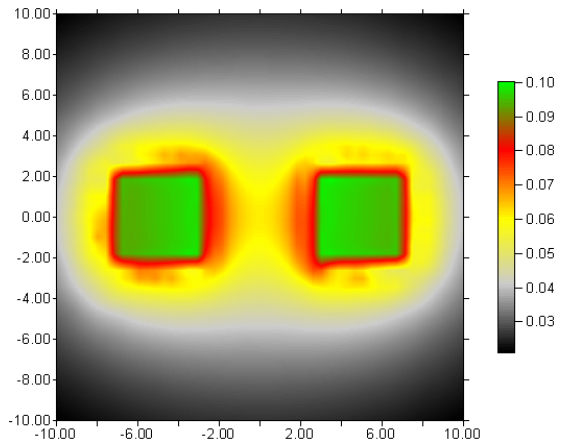

Figura 6.19: Vista em planta dos deslocamentos verticais

representa um aumento de aproximadamente $6,7 \%$ em relação ao bloco isolado calculado no item 6.2. Outra observação importante a ser feita na figura 6.19 é que os blocos se inclinaram para o centro, devido à influência de um sobre o outro. Mesmo pequena esta inclinação introduz excentricidades aos carregamentos aplicados nos blocos, e isto não seria detectado analisando cada bloco isoladamente.

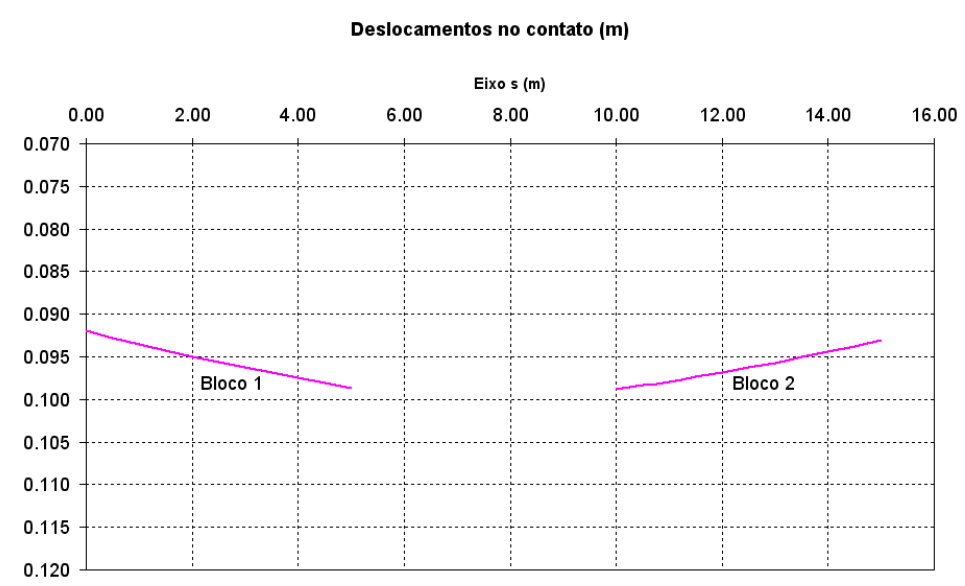

Figura 6.20: Deslocamento vertical na base dos blocos

Para melhor visualização da inclinação dos blocos, foi montado o gráfico da figura 6.20. Este gráfico, para melhor entendimento, contém apenas os nós dos blocos presentes no eixo $s$ (figura 6.17).

Outro resultado relevante é o das tensões de contato que ocorrem entre os blocos e o solo. No gráfico da figura 6.21, estão ilustrados os valores da tensão ao longo do eixo $s$ (figura 6.17). Comparando as tensões obtidas para dois blocos, figura 6.21. com as tensões obtidas no item 6.2 para um bloco, figura 6.6, pode-se concluir que não há diferenças significativas. A assimetria dos resultados, decorrente da malha não simétrica do solo , causa maiores perturbações que a influência dos blocos entre si.

Pode-se concluir que os resultados obtidos neste exemplo são coerentes. Não foi possível reproduzir este exemplo utilizando o programa Ansys, pois este programa é baseado no MEF. Para obter valores coerentes, seria necessário dividir os blocos 


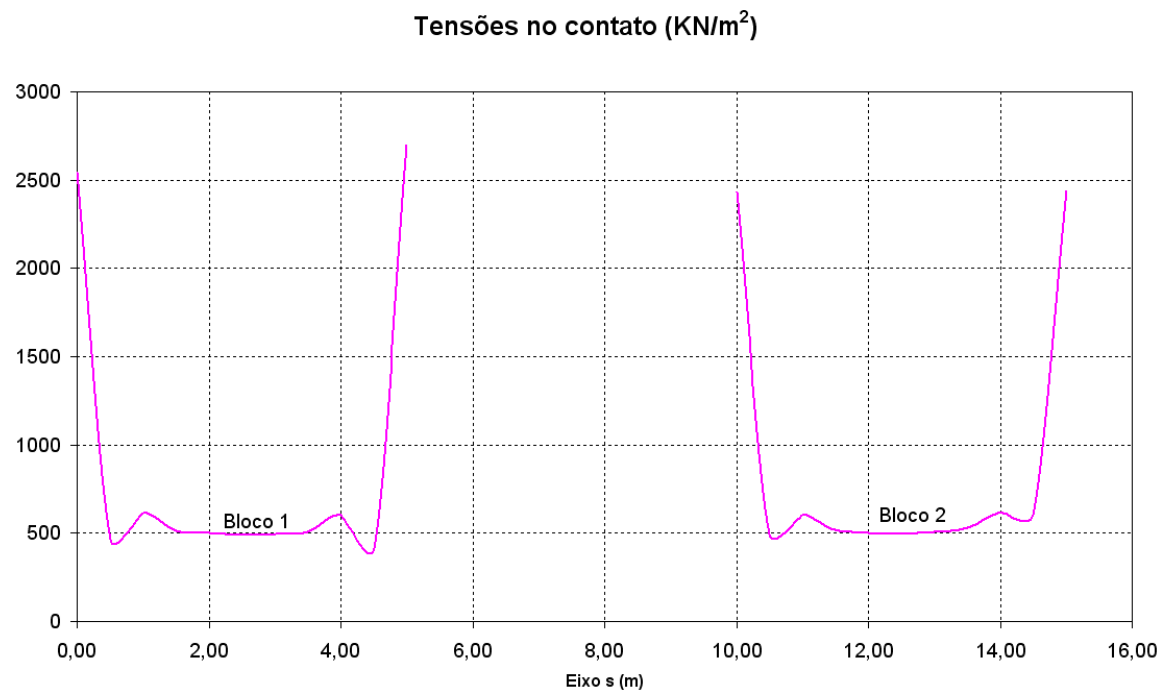

Figura 6.21: Tensão de contato na base dos blocos

e o solo em um grande número de elementos finitos tridimensionais. Isto resultaria em um sistema de equações demasiadamente grande, inviabilizando sua resolução.

\subsection{Interação entre quatro blocos}

O objetivo deste exemplo é avaliar a influência de quatro blocos entre si. Os blocos são iguais, sendo adotados para cada um deles valores iguais aos do item 6.2 . Portanto, as dimensões de cada bloco são de 5 por 5 metros em planta e 1,25 metros de altura. O módulo de elasticidade adotado para os blocos é de $21000000 k \mathrm{~N} / \mathrm{m}^{2}$ e o coeficiente de Poisson de 0,3. O solo, assumido como um semi-espaço infinito, tem módulo de elasticidade igual a $40000 \mathrm{kN} / \mathrm{m}^{2}$ e coeficiente de Poisson igual a 0,3. A carga externa, aplicada verticalmente no topo de cada bloco de forma uniformemente distribuída, é de $1000 \mathrm{kN} / \mathrm{m}^{2}$. A figura 6.22 ilustra a geometria do problema.

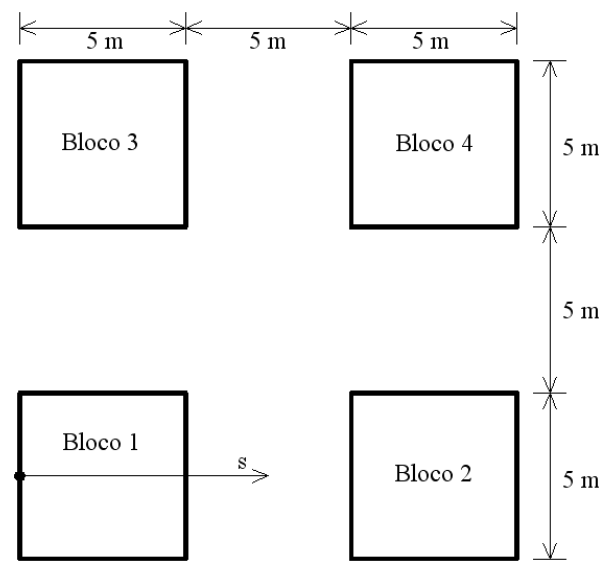

Figura 6.22: Vista em planta

Foi adotada uma distância de 5 metros entre os blocos, conforme ilustrado na 
figura 6.22. O eixo $s$ tem origem na face esquerda do bloco 1 , sendo utilizado como referência em alguns resultados. As unidades de todos os valores apresentados então em $k N$ e metro.

A figura 6.23 apresenta os deslocamentos verticais em metros ocorridos na superfície livre do solo. Pode-se visualizar, pela cor verde, a área do solo onde os blocos estão apoiados. O deslocamento máximo ocorreu nos cantos mais próximos do centro, com valor de aproximadamente 12,8 centímetros. Isto representa uma diferença de aproximadamente $36,1 \%$ em relação ao bloco isolado calculado no item 6.2 e de $27,5 \%$ em relação aos dois blocos calculados no item 6.4. A inclinação para o centro também se tornou mais evidente que aquela detectada no item 6.4. Isto implica em excentricidades mais sensíveis para os carregamentos aplicados nos blocos, tornando este efeito mais relevante.

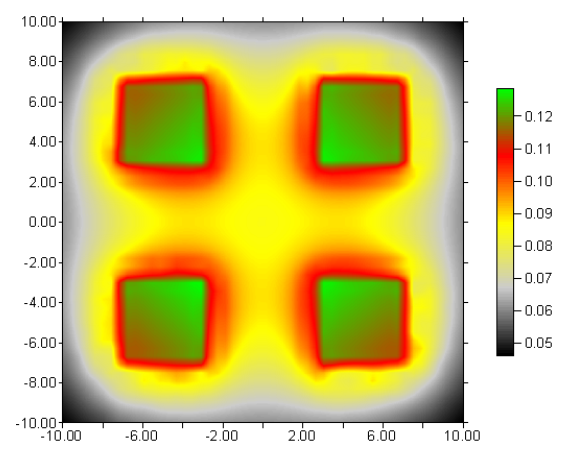

Figura 6.23: Vista em planta dos deslocamentos verticais

O gráfico da figura 6.24 permite visualizar com mais facilidade a inclinação dos blocos 1 e 2. Foram considerados somente os nós pertencentes ao eixo $s$ ilustrado na figura 6.22. Comparando as figuras 6.20 e 6.24, é possível notar uma inclinação mais acentuada ao se considerar quatro blocos. Enquanto a diferença nos deslocamentos das extremidades do bloco 1 da figura 6.20 é de aproximadamente 6,5 milímetros, na figura 6.24 esta diferença atinge 9,5 milímetros. Outra comparação importante a ser feita entre estas figuras se refere à diferença nos valores dos deslocamentos, claramente superiores ao se considerar quatro blocos.

As tensões de contato que ocorrem entre os blocos 1 e 2 e o solo estão ilustradas no gráfico da figura 6.25. São tomados os valores ao longo d eixo $s$ ilustrado na figura 6.22. Assim como ocorreu na comparação dos exemplos dos itens 6.2 e 6.4 , não houve mudanças significativas nas tensões de contato dos blocos com o solo. A influência dos blocos entre si não afeta este resultado sensivelmente. Novamente ocorrem pequenas assimetrias na resposta, o que pode ser justificado pela malha de elementos de contorno não simétrica que foi empregada no solo para a resolução do exemplo.

Pode-se concluir que os resultados obtidos neste exemplo são coerentes. Assim 


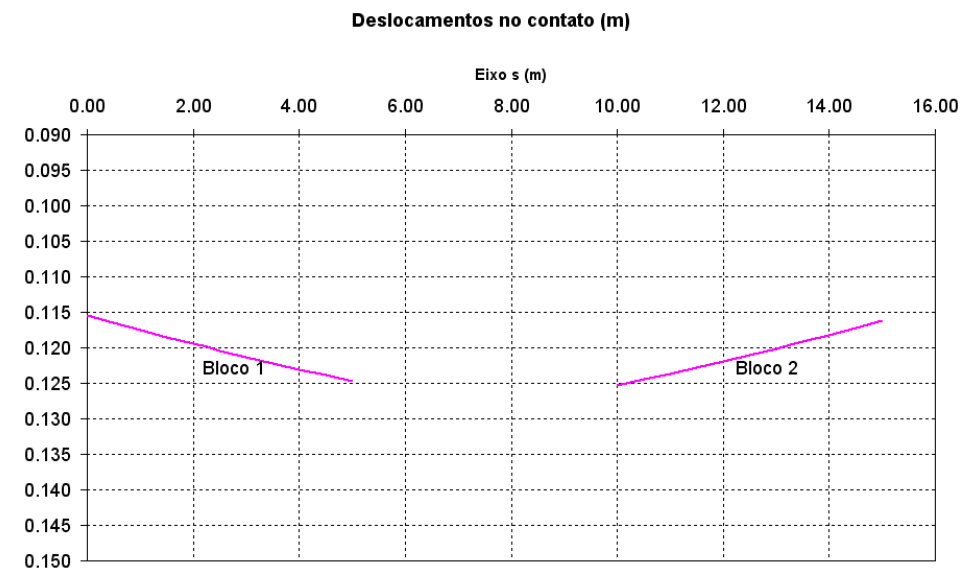

Figura 6.24: Deslocamento vertical na base dos blocos

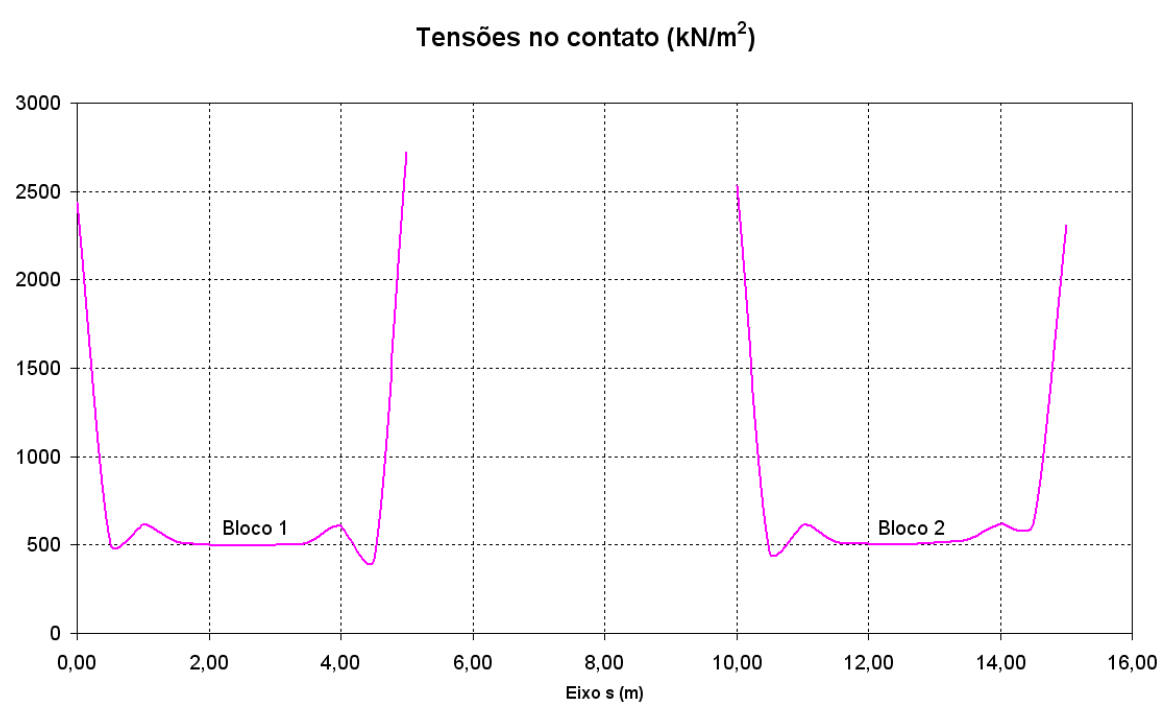

Figura 6.25: Tensão de contato na base dos blocos

como no exemplo do item 6.4 não foi possível reproduzir esta análise no programa Ansys devido ao número elevado de elementos finitos tridimensionais necessários para uma simulação adequada do problema.

\subsection{Edificação apoiada sobre blocos}

Neste exemplo é analisada uma edificação tridimensional de um pavimento apoiada em quatro blocos, conforme ilustrado nas figuras 6.26 e 6.27. São utilizados elementos de casca DKT/FF nas lajes e seis graus de liberade por nó nas barras, diferente da formulação empregada em ALMEIDA (2003a).

A edificação é composta por uma laje apoiada em quatro vigas, as quais são apoiadas nos quatro pilares. Todo o edifício é simulado pelo MEF, sendo a laje modelada pelo elemento de casca DKT/FF e os pilares e vigas por elementos de barra. A distância entre os pilares é de 10 metros, e a altura do pavimento é de 3 


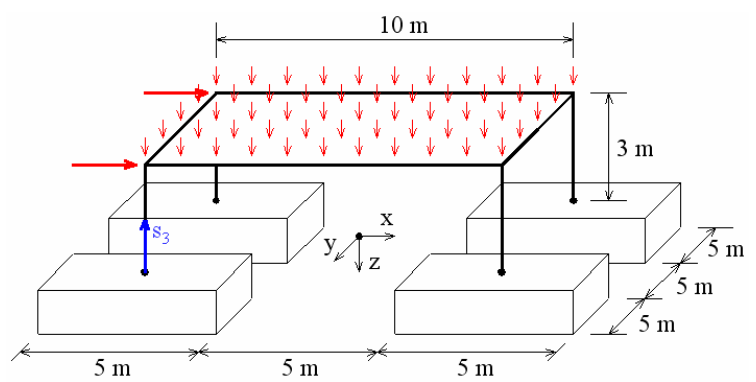

Figura 6.26: Edificação tridimensional em perspectiva

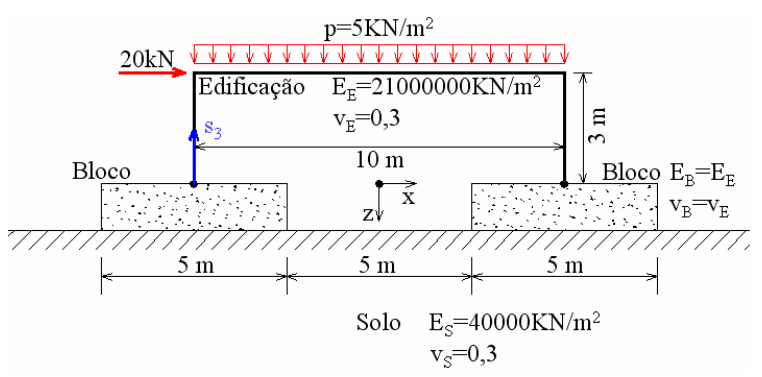

Figura 6.27: Edificação tridimensional em perfil

metros. O eixo $s_{3}$, indicado nas figuras 6.26 e 6.27, tem origem na base de um dos pilares e mesma direção do eixo cartesiano $z$, indicado nas mesmas figuras.

Os blocos utilizados para apoiar a edificação são idênticos aos da seção 6.5. Portanto, as dimensões de cada bloco são de 5 por 5 metros em planta e 1,25 metros de altura. As propriedades físicas adotadas para os blocos são módulo de elasticidade de $21000000 k N / m^{2}$ e coeficiente de Poisson 0,3. A distância entre os blocos é de 5 metros, tanto na direção $x$ como na direção $y$. O solo, assumido como um semiespaço infinito, tem módulo de elasticidade igual a $40000 \mathrm{kN} / \mathrm{m}^{2}$ e coeficiente de Poisson 0,3. Também neste exemplo será utilizado o eixo $s$, indicado na figura 6.28.

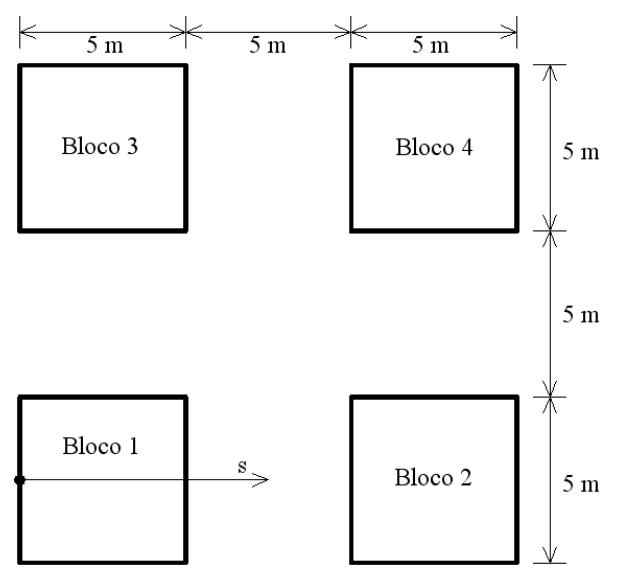

Figura 6.28: Vista em planta dos blocos

A espessura adotada para a laje foi de 0,3 metro e uma seção transversal quadrada 
com 0,5 metro de lado para todas as vigas e pilares, conforme ilustrado na figura 6.29. Os eixos $x$ e $y$ desta figura têm a mesma direção e orientação dos eixos $x$ e $y$ da figura 6.26 .

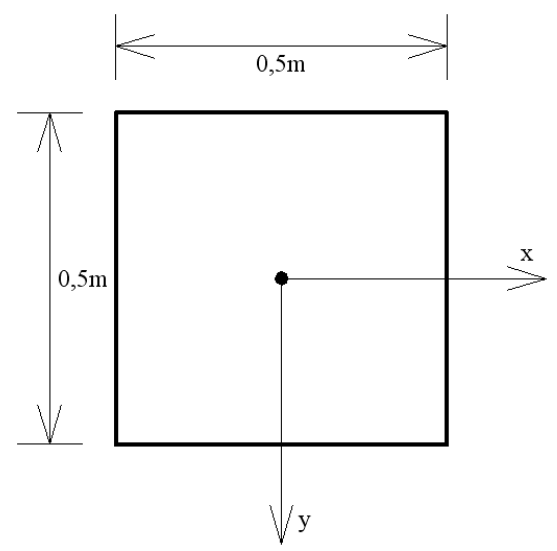

Figura 6.29: Seção transversal dos pilares e vigas

A geometria da laje pode ser melhor visualizada na figura 6.30. Nesta figura, o eixo $s_{1}$ é alinhado ao eixo $x$ da figura 6.26 e o eixo $s_{2}$ é alinhado ao eixo $y$. Foi adotado para a laje, os pilares e as vigas um módulo de elasticidade longitudinal igual a $21000000 \mathrm{kN} / \mathrm{m}^{2}$ e coeficiente de Poisson 0,3. Foi aplicado na laje um carregamento vertical uniformemente distribuído e igual a $5 \mathrm{kN} / \mathrm{m}^{2}$, além de duas cargas horizontais na direção do eixo $x$. Estas cargas horizontais podem ser visualizadas nas figuras 6.26 e 6.27, e têm o valor de $20 k N$ cada uma.

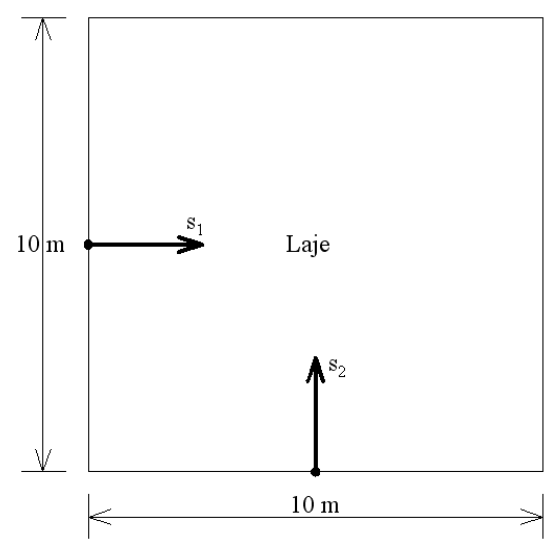

Figura 6.30: Geometria da laje

Em todos os resultados apresentados neste exemplo, as unidades estão em metros e $k N / m^{2}$.

A figura 6.31 apresenta os deslocamentos verticais em metros ocorridos na superfície livre do solo. A cor verde indica as áreas que mais se deslocaram, enquanto as cores escuras indicam as áreas que menos se deslocaram. É possível notar que a inclinação dos blocos é de dentro para fora, comportamento inverso ao observado 
na figura 6.23 do item 6.5. Este comportamento pode ser explicado pelo momento fletor que é transmitido da laje para os pilares e destes para os blocos. Outra observação que pode ser feita é que os blocos da direita afundam um pouco mais que os da esquerda. Isto é conseqüência do efeito do carregamento horizontal aplicado da esquerda para a direita, causando um pequeno alívio nos blocos da esquerda e uma pequena sobre-carga nos blocos da direita. O deslocamento máximo ocorreu nos cantos mais afastados do centro, com valor de aproximadamente 0,075 centímetros.

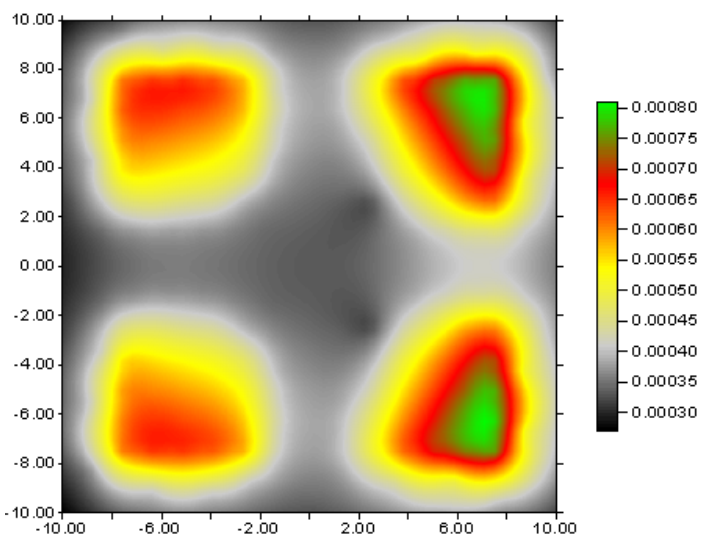

Figura 6.31: Vista em planta dos deslocamentos verticais

Para melhor visualizar a diferença nos deslocamentos dos blocos da direita e esquerda, foi montado o gráfico da figura 6.32. Este gráfico contém os deslocamentos verticais ocorridos nos blocos 1 e 2 ao longo do eixo $s$ (figura 6.28). Além da inclinação dos blocos, é possível notar que o bloco da direita se inclina sensivelmente mais que o bloco da esquerda. Esta diferença acontece devido à carga horizontal aplicada do lado esquerdo da edificação, causando uma maior solicitação dos blocos da direita.

As tensões de contato que ocorrem entre os blocos 1 e 2 e o solo estão ilustradas no gráfico da figura 6.33. São tomados os valores ao longo do eixo $s$ ilustrado na figura 6.28. É possível observar que as tensões de contato na base do bloco 1 não sofrem tanta variação quanto as tensões na base do bloco 2 . Isso pode ser considerado coerente, após observar na figura 6.32 que o bloco 2 se inclina mais que o bloco 1 .

Estão ilustrados, na figura 6.34, os deslocamentos calculados na direção z para os nós localizados sobre o eixo $s_{1}$ da laje da edificação. Além da edificação apoiada sobre o conjunto formado pelos blocos e o solo, também foi calculada esta mesma edificação com a base dos pilares restringida. Ou seja, restringiu-se os seis graus de liberdade existentes na extremidade inferior de cada pilar.

É possível observar que a edificação, quando apoiada no conjunto formado pelos blocos e o solo, se desloca mais do que quando tem sua base restringida. Isto é 


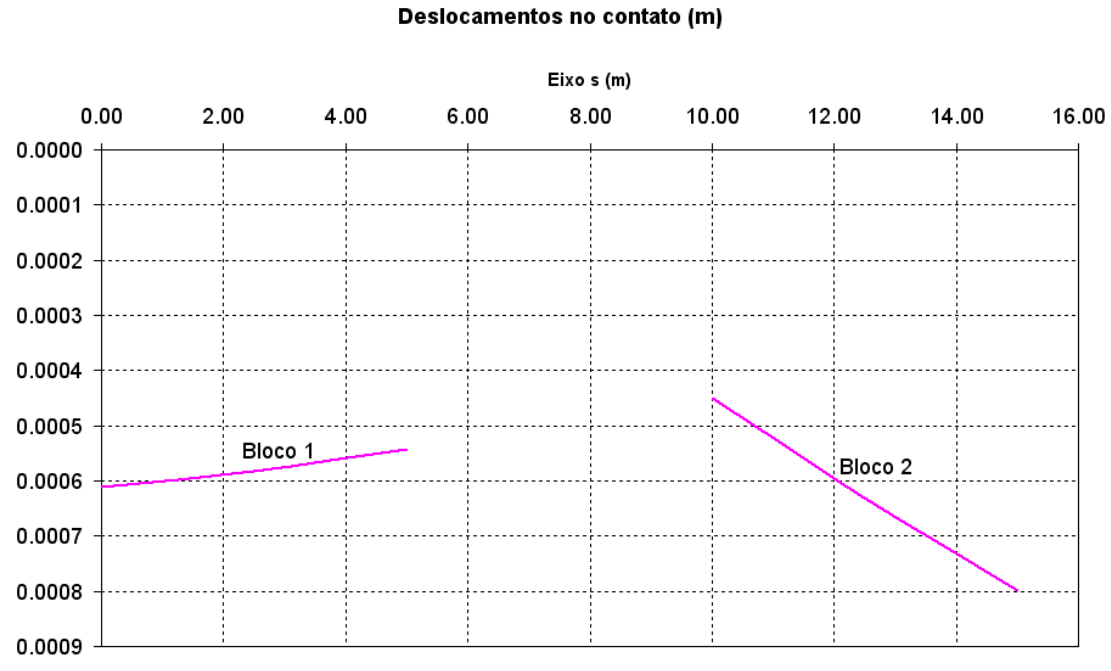

Figura 6.32: Deslocamento vertical na base dos blocos

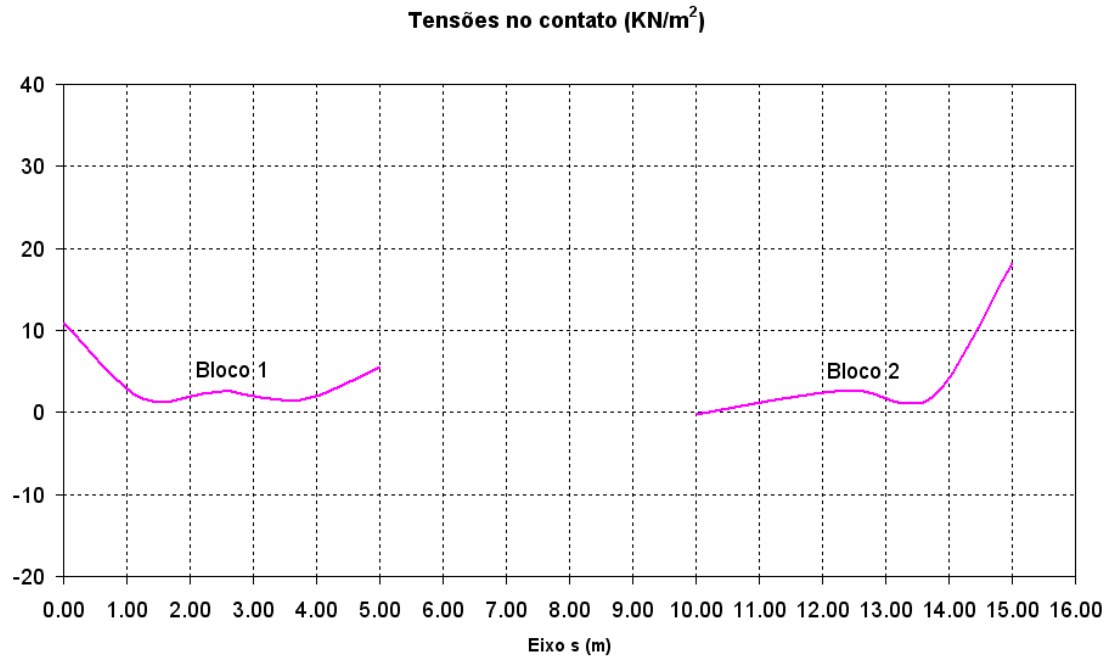

Figura 6.33: Tensão de contato na base dos blocos

previsível, uma vez que os deslocamentos ocorridos no solo e nos blocos se propagam para o edifício. Esta diferença, ao longo do gráfico, é de cerca de 1 milímetro.

O gráfico da figura 6.35 também contém os deslocamentos calculados na direção $z$ para os nós da laje, só que para o eixo $s_{2}$. Da mesma forma, foram colocados no mesmo gráfico os deslocamentos calculados com a base dos pilares restringida.

Nota-se novamente a diferença entre as curvas de aproximadamente 1 milímetro. Apesar de semelhantes, as figuras 6.34 e6.35não são iguais. O deslocamento máximo é o mesmo, ocorrido no centro da laje onde os eixos $s_{1}$ e $s_{2}$ se cruzam, no entanto, os deslocamentos mínimos ocorridos nas bordas da laje são diferentes. A variação no deslocamento ao longo do eixo $s_{1}$ é maior que no eixo $s_{2}$, o que permite concluir que a flexão da laje é mais intensa na direção do eixo $x$. Esta diferença pode ser explicada pelo efeito das cargas horizontais aplicadas na edificação na direção $x$.

O momento fletor na direção $x$ ao longo do eixo $s_{1}$ está representado na figura 


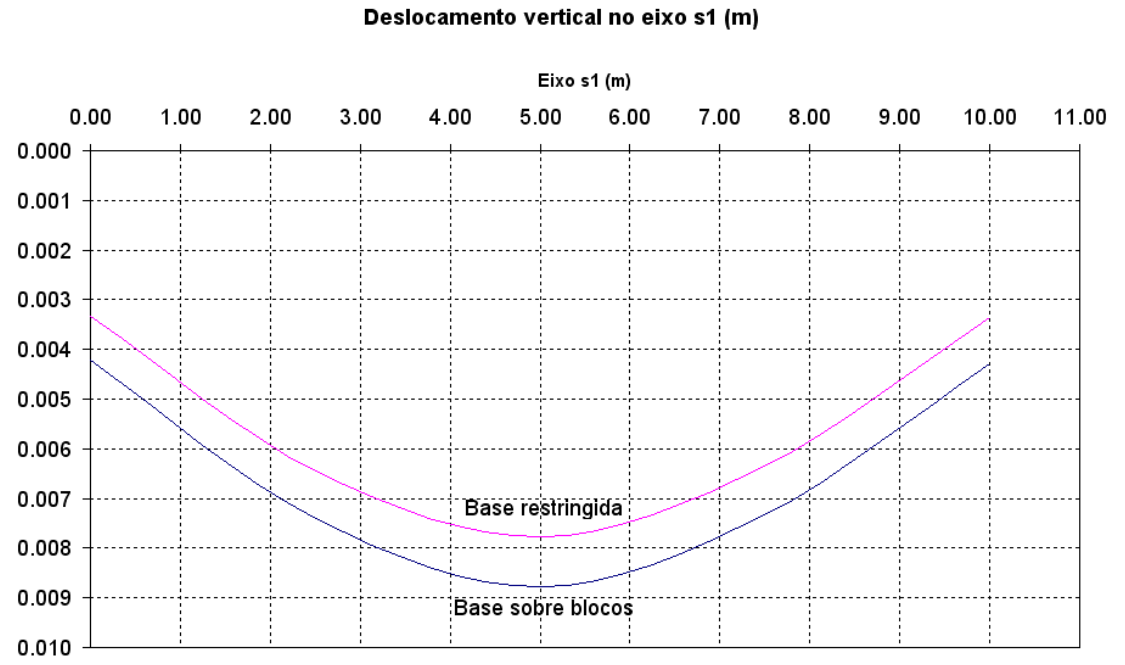

Figura 6.34: Deslocamento vertical no eixo $s_{1}$

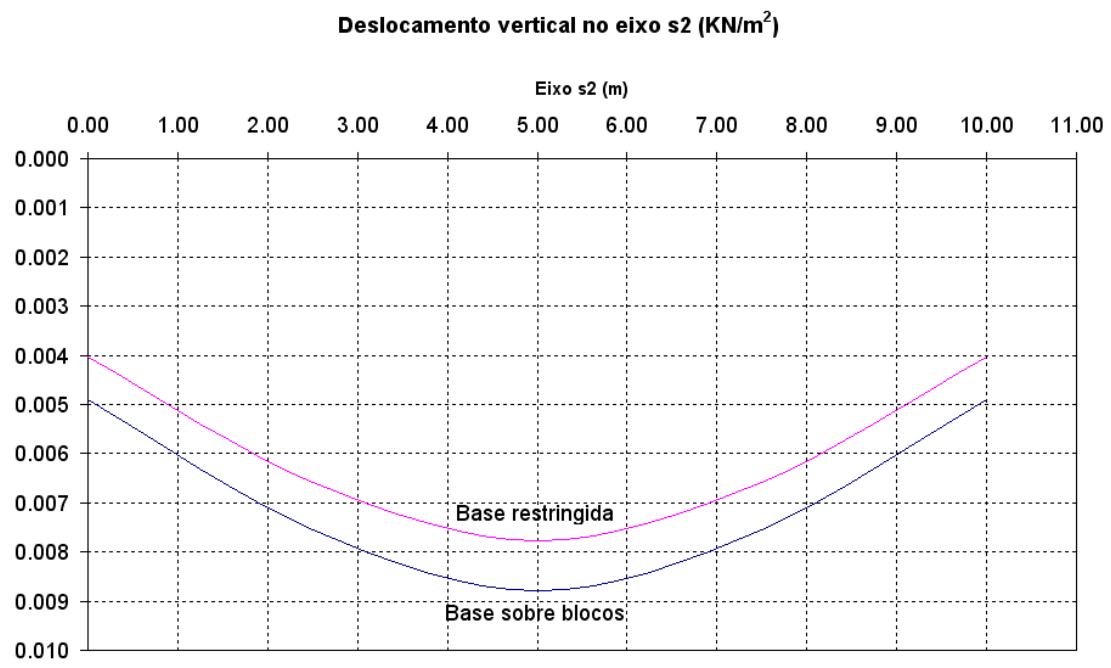

Figura 6.35: Deslocamento vertical no eixo $s_{2}$

6.36, sendo analisado também para os dois tipos de fundação.

Algo interessante de ser observado na figura 6.36 é que o momento fletor resultou maior quando foi considerada a interação do solo com a estrutura. Em outras palavras, quando a fundação é o conjunto solo/blocos o momento $M X$ resulta superior ao calculado quando a fundação está restringida. Com isto, conclui-se que é importante a consideração da interação do solo com a estrutura. Caso esta interação fosse ignorada, o esforço adicional decorrente da flexibilidade da fundação não seria detectado.

Pode-se chegar à mesma conclusão analisando-se o gráfico da figura 6.37, no qual estão apresentados os valores do momento fletor na direção $y$ ao longo do eixo $s_{1}$. A diferença entre os valores das curvas nesta figura ultrapassa $5 \%$ nos apoios, podendo ser considerada relevante. Caso o solo fosse mais flexível, esta diferença seria ainda maior. 
Momento MX no eixo s1 (KNm)

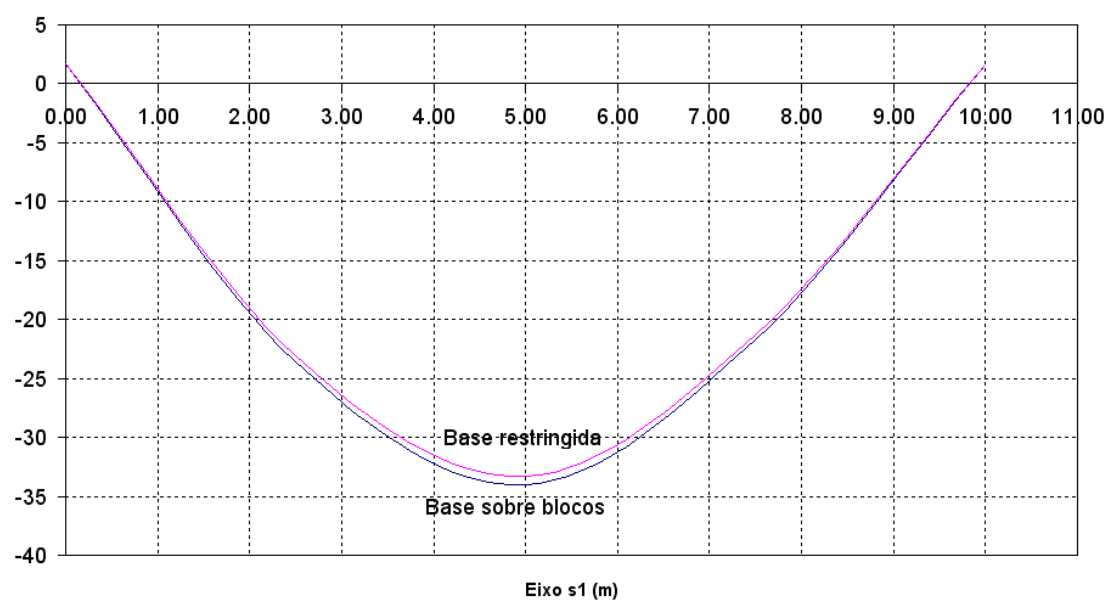

Figura 6.36: Momento MX no eixo $s_{1}$

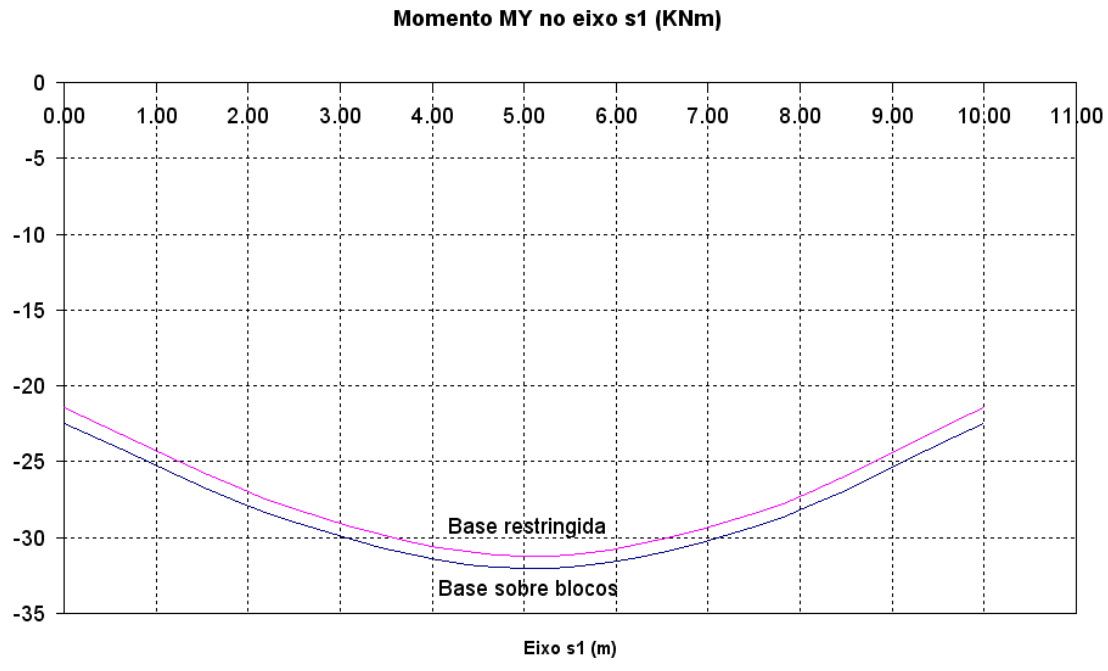

Figura 6.37: Momento MY no eixo $s_{1}$

Um outro dado a ser apresentado é o dos deslocamentos horizontais na direção $x$ que ocorrem ao longo de um dos pilares. O pilar escolhido é o que está apoiado sobre o bloco 1 , indicado na figura 6.28. Ao longo deste pilar foi definido o eixo $s_{3}$, ilustrado nas figuras 6.26 e 6.27. O deslocamento na direção $x$ ao longo do pilar está apresentado no gráfico da figura 6.38 .

Quando o pilar é conectado ao bloco, que por sua vez está apoiado no solo, nota-se que o deslocamento em sua base é diferente de zero. Já para o caso do pilar com base restringida, conforme previsto, a base tem deslocamento nulo. Mesmo com um deslocamento para a esquerda em sua base, o pilar conectado ao bloco apresenta um deslocamento para a direita no topo claramente superior ao caso do pilar com base restringida. Esta diferença é superior a 50\%, tomando como referência o deslocamento do pilar de base restringida. Observando este comportamento, conclui-se novamente que os efeitos da interação do solo com a estrutura não podem 


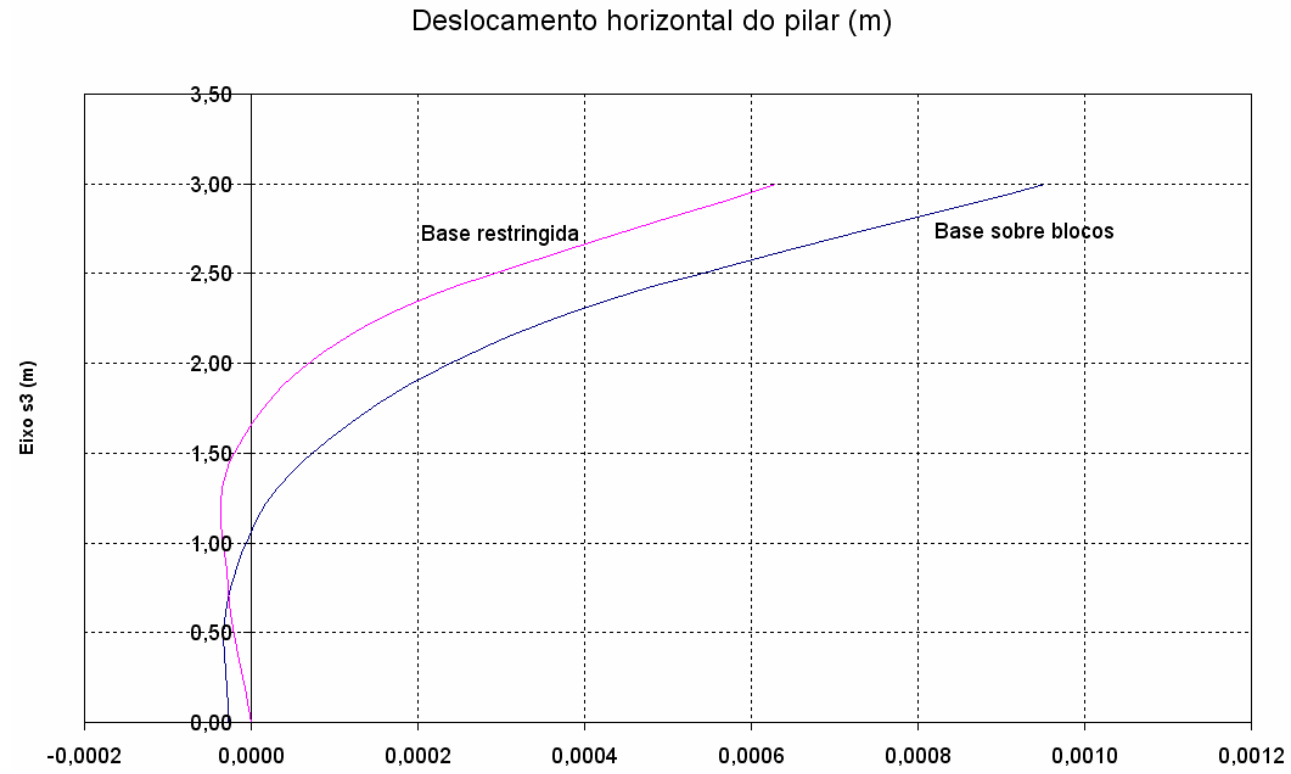

Figura 6.38: Deslocamento horizontal do pilar

ser desprezados. Como os deslocamentos são diferentes em cada caso, pode-se concluir que os esforços também são. Portanto, caso a interação solo/estrutura não seja considerada, não é possível calcular com exatidão os esforços que solicitam o pilar.

Este exemplo apresenta resultados coerentes, e a partir dele é possível perceber a importância da consideração da interação do solo com a estrutura. Não foram apresentadas comparações com outros autores devido à escassez de trabalhos que analisem a interação do solo com a estrutura em abordagem tridimensional.

\subsection{Considerações finais}

Este capítulo apresentou uma série de resultados obtidos a partir do programa desenvolvido por ALMEIDA (2003a) e das extensões desenvolvidas neste trabalho de mestrado. Grande parte dos resultados apresentados representam contribuições originais, devido à escassez na literatura de trabalhos que analisem a interação do solo com a estrutura de forma semalhante à realizada aqui. 


\section{Capítulo 7}

\section{Conclusões}

\subsection{Observações finais}

Para o desenvolvimento deste trabalho, foi feita uma revisão bibliográfica voltada aos trabalhos que tratam da interação do solo com a estrutura. A maior parte das publicações apresenta modelos com algumas limitações para a simulação do sistema fundação/solo, tais como a consideração de um meio homogêneo, isotrópico e semi-infinito. Além disto, a grande maioria dos trabalhos emprega formulações em duas dimensões, o que dificulta a investigação de casos reais de engenharia. Estas limitações podem ser justificadas pelo grande volume de dados que devem ser gerados em uma análise tridimensional de um problema tão complexo quanto a interação solo/estrutura.

A ferramenta numérica que se apresenta como a melhor opção para a simulação tridimensional do solo semi-infinito é o método dos elementos de contorno (MEC), devido às propriedades de suas funções ponderadoras que permitem a consideração de condições de contorno aplicadas a grandes distâncias. Além disto, pelo MEC, é possível representar um problema tridimensional por meio de um modelo em duas dimensões. Isto diminui consideravelmente o volume de dados gerado na análise.

Aplicando o MEC em análise tridimensional, este trabalho apresentou uma ferramenta computacional para simulação de problemas de interação do solo com a estrutura. O ponto de partida foi o programa desenvolvido por ALMEIDA (2003a), tendo sido revisada a teoria envolvida em sua implementação.

O programa original de ALMEIDA (2003a) emprega o método dos elementos de contorno (MEC) tridimensional, simulando um solo apoiado em uma superfície de deslocamento nulo e dividido em camadas, podendo estas ser enrijecidas por um número qualquer de estacas. Neste solo foi empregado o Método da Rigidez Sucessiva (MRS) apresentado por MAIER \& NOVATI (1987), aplicado a um meio tridimensional e enrigecido por elementos de fundação. 
A superestrutura, que pode ser até um edifício, é simulada por três tipos de modelos numéricos. O primeiro são elementos finitos triangulares DKT/FF, que podem ser empregados em uma fundação do tipo radier para o edifício. O segundo são elementos finitos reticulares, nos quais é desprezado o efeito da torção, empregados nas vigas e pilares do edifício. Por fim, as lajes do edifício são modeladas por membranas rígidas.

Combinando as formulações do MEC e do MEF, o programa de ALMEIDA (2003a) permite estudar a interação do solo com a estrutura resolvendo um único sistema de equações que representa toda a estrutura.

A primeira extensão implementada neste trabalho no programa de ALMEIDA (2003a) foi permitir a simulação de blocos tridimensionais e modelados pelo MEC apoiados sobre o solo. A formulação utilizada se baseia em técnicas de sub-regiões do MEC, aplicando condições de equilíbrio de forças e compatibilidade de deslocamentos nas superfícies de contato entre os diferentes meios.

Alguns exemplos envolvendo blocos sobre o solo foram resolvidos. Alguns dos resultados obtidos são contribuições originais, pois não há publicações na bibliografia nas quais são apresentadas as tensões de contato que ocorrem entre blocos tridimensionais e o solo. Outro dado interessante é a influência de um bloco no outro, impossível de ser detectada analisando-se cada bloco isoladamente.

Pode-se chegar a uma conclusão interessante a partir da diferença que ocorre nos deslocamentos do bloco ao considerar ou não uma superfíciede deslocamentos nulos. Caso dois blocos estivessem servindo de fundação para uma edificação, ambos idênticoa, submetidos à mesma carga e apoiadono mesmo tipo de solo, e sob apenas um deles houvesse a camada de deslocamentos nulos, o recalque diferencial que ocorreria entre estes blocos seria relevante. Esta diferença no recalque produziria esforços não previstos na edificação em questão, podendo prejudicar sua estrutura.

Foi verificada uma boa coerência nos exemplos nos quais são analisados blocos apoiados em um solo sem estacas. Quando é considerado um número maior de blocos, dois ao invés de quatro, torna-se mais evidente a influência entre eles. Este fato foi observado pela inclinação estabelecida em cada um deles, mais acentuada quando se aumenta o número de blocos. Assim, é possível concluir que a formulação empregada na análise de um número qualquer de blocos apoiados sobre o solo é adequada. Desta forma, o objetivo de acoplar ao solo um número qualquer de blocos tridimensionais foi cumprido.

$\mathrm{Na}$ parte final do trabalho, foi acoplado ao sistema composto por blocos e o solo um edifício tridimensional modelado pelo MEF. Esate edifício é diferente do considerado na formulação de ALMEIDA (2003a), pois as lajes são simuladas por elementos DKT/FF e os pilares são modelados por elementos reticulares com seis graus de liberdade por nó. A formulação utilizada para acoplar o edifício ao con- 
junto solo/blocos foi a mesma empregada em ALMEIDA (2003a) no acoplamento placa/solo. Para que isto fosse possível a conecção dos pilares com os blocos foi intermediada por uma placa flaxível modelada por elementos DKT/FF, tal como a placa considerada em ALMEIDA (2003a).

No último exemplo foi considerada uma edificação de um pavimento, apoiando seus pilares em blocos e apoiando os blocos no solo. Nete exemplo foi possível estudar de forma mais abrangente a interação do solo com a estrutura, apresentando resultados que comprovam sua importância. A inclinação estabelecida nos blocos devido aos momentos transmitidos da estrutura é um dado interessante, pois nos exemplos anteriores nos quais foram considerados blocos esta inclinação foi diferente. As diferenças detectadas entre dois blocos nos deslocamentos e tensões de seu contato com o solo foram explicadas pelo carregamento horizontal aplicado à edificação. Os resultados apresentados para a estrutura foram deslocamentos na laje, deslocamentos em um pilar e momentos fletores na laje, comparando os valores calculados para a fundação solo/blocos com valores calculados para uma fundação rígida. A análise das diferenças em cada caso levou à conclusão de que os efeitos da interação do solo com a estrutra não podem ser desprezados. Este estudo consiste em mais uma contribuição original.

De forma geral, os resultados apresentados demonstraram a consistência do programa com resultados coerentes. Alguns destes resultados foram comparados com o programa Ansys ou publicações de outros autores, validando a formulação empregada. Em alguns exemplos não foram apresentadas comparações devido à escassez existente na literatura de resultados semelhantes. Portanto, este trabalho preenche uma lacuna existente nas pesquisas sobre interação do solo com a estrutura, que é a simulação deste problema de forma mais completa e em abordagem tridimensional.

Por fim, este trabalho apresentou uma poderosa e versátil ferramenta para o estudo da interação do solo com a estrutura. Como principais contribuições deste trabalho, podem-se citar as análises das tensões de contato estabelecidas entre blocos tridimensionais e o solo, da influência entre blocos e dos resultados obtidos para um edifício apoiado sobre blocos.

\subsection{Propostas para trabalhos futuros}

A seguir, são enumeradas propostas para trabalhos futuros que podem dar continuidade ao programa computacional aqui desenvolvido.

1. Modelar o solo empregando uma formulação semi-analítica, eleminando o problema com os nós duplos;

2. Substituir as estacas tridimensionais por um modelo mais simplificado, de 
forma a reduzir o tempo de processamento necessário para calcular estruturas mais complexas;

3. Considerar o deslizamento das estacas em relação ao maciço;

4. Empregar modelos reológicos mais completos no solo considerando, por exemplo, não-linearidade física;

5. Empregar modelos de não-linearidade geométrica na superestrutura. 


\section{Referências Bibliográficas}

ALMEIDA, F. P. A (2003). Aplicação do acoplamento entre o MEC e o MEF para o estudo da interação dinâmica elastoplástica entre o solo e estruturas. 285p. Tese (Doutorado) - Escola de Engenharia de São Carlos, Universidade de São Paulo, São Carlos, Brasil. 2003.

ALMEIDA, V. S (2003). Análise da interação solo não-homogêneo/estrutura via acoplamento MEC/MEF. 192p. Tese (Doutorado) - Escola de Engenharia de São Carlos, Universidade de São Paulo, São Carlos, Brasil. 2003.

ALMEIDA, V. S.; PAIVA, J. B (2004). Análise da interação solo nãohomogêneo/fundação empregando o MEC juntamente com a técnica da rigidez sucessiva. In: XXXI JORNADAS SUD-AMERICANAS DE INGENIERÍA ESTRUCTURAL, 2004, Mendoza, Argentina. Anais... CD-ROM.

ANTUnES, H. M. C. C.; IWAMOTO, R. K (2000). Comparação entre resultados observados in situ e modelos numéricos para a interação solo-estrutura. In: SIMPÓSIO DE INTERAÇÃO ESTRUTURA-SOLO EM EDIFÍCIOS, 2000, São Carlos. Anais... SGS/EESC-USP, 2000.

AOKI, N.; LOPES, F. R (1975). Estimating stress and settlements due to deep foundation. In: CONF. PANAM, 1975, Buenos Aires. Anais... Buenos Aires: HUELLA - Estudio Grafico. v.5, p.377-386.

ASSAN, A. E (2003). Método dos elementos finitos primeiros passos. 2.ed. Campinas, Brasil: UNICAMP.

BANERJEE, P. K (1976). Integral equation methods for analysis of piece-wise nonhomogeneous three-dimensional elastic solids of arbitrary shape. International Journal of Mechanical Sciences, England, v.18, p.293-303.

BANERJEE, P. K (1978). Analysis of axially and laterally loaded pile groups. In: SCOTT, C. (Ed.). Developments in Soil Mechanics - I. U. K.: Applied Science Publishers. p.317-343. 
BANERJEE, P. K.; DAVIES, T. G (1977). Analysis of pile groups embedded in Gibson soil. In: INT. CONF. SOIL MECHS FDN ENGNG., 9., 1977, Tokyo. Proceedings. . v.1, p.381-386.

BARRETTO, S. F. A (1995). Análise elastodinâmica de placas através do método dos elementos de contorno interação solo-estrutura. 130p. Tese (Doutorado) - Escola de Engenharia de São Carlos, Universidade de São Paulo, São Carlos, Brasil. 1995.

BATHE, J. K (1982). Finite element procedures in engineering analysis. Englewood Cliffs: Prentice-Hall.

BATOZ, J. L (1980). A study of tree-node triangular plate bending elements. International Journal of Numerical Methods in Engineering, England, v.15, p.17711812.

BEER, G.; MEEK, J. L (1981). Coupled finite element-boudary element analysis of finite domain problems in geomecanics. In: HINTON, E.; BETTESS, P.; LEWIS, R. W. (Ed.). Numerical methods for coupled problems. Swansea: Pineridge Press. p.605-629.

BERGAN, P. G.; FELIPPA, C. A (1985). A triangular membrane element with rotacional degrees of freedom. Computer Methods in Applied Mechanics and Engineering, Switzerland, v.50, p.25-69.

BEZERRA, D. P (1995). Análise de estruturas tridimensionais de edifícios altos considerando a rigidez transversal à flexão das lajes. 138p. Dissertação (Mestrado) - Escola de Engenharia de São Carlos, Universidade de São Paulo, São Carlos, Brasil. 1995.

BOOKER, J. R.; CARTER, J. P.; SMALL, J. C (1989). Some recent applications of numerical methods to geotechnical analysis. Computers and Structures, England, v.31, n.1, p.81-92.

BOUSSINESQ, J (1885). Aplications des potentiels à l'études de l'équilibre et du mouviment des solides élastiques. Paris: Gauthier-Villars.

BREBBIA, C. A.; DOMINGUEZ, J (1989). Boundary elements an introductory course. London: Computational Mechanics Publications.

BREBBIA, C. A.; GEORGIOU, P (1979). Combination of boundary and finite elements in elastostatic. Applied Mathematical Modelling, New York, v.3, p.212220.

BURMISTER, D. M (1945). The general theory of stresses and displacements in layered systems 1. Journal of applied physics, New York, v.16, p.89-96. 
BURMISTER, D. M (1945). The general theory of stresses and displacements in layered systems 3. Journal of applied physics, New York, v.16, p.296-302.

BUTTERFIELD, R.; BANERJEE, P. K (1971). The problem of pile group-pile cap interaction. Géotechnique, London, England, v.21, n.2, p.135-142.

CHAN, K. S.; KARASUDHI, P.; LEE, S. L (1974). Force at a point in the interior of a layered elastic half-space. Int. J. Solids Structs., v.10, p.1179-1199.

CHEUNG, Y. K.; THAM, L. G.; GUO, D. J (1988). Analysis of pile group by infinite layer method. Géotechnique, London, England, v.38, n.3, p.415-431.

CHEUNG, Y. K.; ZIENKIEWICZ, O. C (1965). Plates and tanks on elastic foundations - an application of finite element method. Int. J. Solids Structs, v.1, p.451461.

CHIN, J. T.; CHOW, Y. K (1990). Numerical analysis of axially loaded vertical piles and pile groups. Computers and Geotechnics, England, v.9, p.273-290.

CHOW, Y. K.; TEH, C. I (1991). Pile-cap-pile-group interaction in nonhomogeneous soil. Journal of Geotechnical Engineering, New York, v.117, n.11, p.1655-1668.

DAVIES, T. G.; BANERJEE, P. K (1978). Displacement field due to a point load at the interface of a two-layer elastic half-space. Géotechnique, London, England, v.28, n.1, p.43-56.

DUARTE, C. A.; BABUSCA, I.; ODEN, J. T (2000). Generalized finite element methods for three-dimensional structural mechanics problems. Computers and Structures, England, v.77, p.215-232.

FERRO, N. C. P (1998). Uma combinação MEC/MEF para análise de fundações enrigecidas por estacas. Tese (Doutorado) - Escola de Engenharia de São Carlos, Universidade de São Paulo, São Carlos, Brasil. 1998.

FRASER, R. A.; WARDLE, L. J (1976). Numerical analysis of rectangular rafts on layered foundations. Géotechnique, London, England, v.26, p.613-630.

GUSMÃO, A. D (1990). Estudo da Interação Solo-estrutura e sua influência em recalques de edificações. 165p. Tese (Doutorado) - Universidade Federal do Rio de Janeiro, COPPE. 1990.

HETENYI, M. A (1950). A general solution for the bending of beams on an elastic foundations of arbitrary continuity. Journal of Applied Physics, New York, v.21, p. $55-58$. 
HOLANDA, O. G. J (1998). Interação solo-estrutura para edifícios de concreto armado sobre fundações diretas. 191p. Dissertação (Mestrado) - Escola de Engenharia de São Carlos, Universidade de São Paulo, São Carlos, Brasil. 1998.

IWAMOTO, R. K (2000). Alguns aspectos dos efeitos da interação solo-estrutura em edifícios de múltiplos andares com fundação profunda. 140p. Dissertação (Mestrado) - Escola de Engenharia de São Carlos, Universidade de São Paulo, São Carlos, Brasil. 2000.

JORDÃO, D. R (2003). Estabilidade global de edifícios sobre fundações profundas, considerando a interação estrutura - solo. 142p. Dissertação (Mestrado) - Escola de Engenharia de São Carlos, Universidade de São Paulo, São Carlos, Brasil. 2003.

KERR, A. D (1964). Elastic and viscoelastic foundation models. Journal Applied Mechanic Transactions, ASME, v.31, p.491-498.

KERR, A. D (1965). A study of a new foundation model. Acta Mechanic, v.1, p.135147.

LEE, C. (1993)Y. Pile group settlement analysis by hybrid layer approach. Journal of Geotechnical Engineering, New York, v.119, n.6, p.984-997.

LEE, C. Y.; SMALL, J. C (1991). Finite Layer Analysis of Laterally Loaded Piles in Cross-Anisotropic Soils. International Journal for Numerical Methods in Geomechanics, England, v.15, p.785-808.

LEITE, L. G. S.; CODA, H. B.; VENTURINI, W. S (2001). Interação entre domínios bidimensionais e barras através do método dos elementos de contorno. In: XXI CILAMCE- CONGRESSO ÍBERO LATINO DE MÉTODOS COMPUTACIONAIS EM ENGENHARIA, 2001, Campinas. Anais... UNICAMP. p.1-13.

LORENTZ, R. G (1985). Alguns aspectos na interação estrutura-solo-estrutura. 114p. Dissertação (Mestrado) - Pontifícia Universidade Católica do Rio de Janeiro, Rio de Janeiro, Brasil. 1985.

LOVE, A. E. H (1944). A treatise on the mathematical theory of elasticity. 4.ed. New York: Dover.

MAIER, G.; NOVATI, G (1987). Boundary element elastic analysis by a successive stiffness method. International Journal for Numerical and Analytical Methods in Geomechanics, England, v.11, p.435-447.

MATTES, N. S.; POUlOS, H. G (1969). Settlement of single compressible pile. Journal of the Soil Mechanics and Foundations Division, v.95, n.1, p.189-207. 
MEEK, J. L (1988). BECOUP - A program for coupled boundary and finite element analysis in 3D elastostatics. In: BREBBIA, C. A. (Ed.). Boundary elements X: stress analysis. Southampton: CML Publ., 1988. v.3, p.639-655.

MELLO, G. J. T (1984). Interação inercial solo-estrutura para subleitos estratificados. 163p. Dissertação (Mestrado) - Pontifícia Universidade Católica do Rio de Janeiro, Rio de Janeiro, Brasil. 1984.

MENDONÇA, A. V (1997). Análise da interação placa-estaca-solo via combinação do método dos elementos finitos com o método dos elementos de contorno. 151p. Dissertação (Mestrado) - Escola de Engenharia de São Carlos, Universidade de São Paulo, São Carlos, Brasil. 1997.

MENDONÇA, A. V.; PAIVA, J. B (2000). A boundary element method for the static analysis of raft foundations on piles. Engineering analysis with boundary elements, England, v.24, p.237-247.

MENDONÇA, A. V.; PAIVA, J. B (2003). A elastostatic FEM/BEM analysis of vertically loaded raft and piled raft foundation. Engineering analysis with boundary elements, England, v.27, p.919-933.

MESQUITA, A. D (1998). Uma formulação do método dos elementos finitos aplicada á analise elastoplástica de cascas. 144p. Dissertação (Mestrado) - Escola de Engenharia de São Carlos, Universidade de São Paulo, São Carlos, Brasil. 1998.

MESQUITA, A. D.; CODA, H. B (2000). Análise viscoelástica da interação solo-estrutura, representação bidimensional. In: SIMPÓSIO DE INTERAÇÃO ESTRUTURA-SOLO EM EDIFÍCIOS, 2000, São Carlos. Anais... SET/EESCUSP.

MINDLIN, R. D (1936). Force at a point in the interior of a semi-infinite solid. $J$. Physics, v.7, p.195-202.

MOURA, A. R. L. U (1995). Interação solo-estrutura em edifícios. 135p. Dissertação (Mestrado) - Universidade Federal de Pernambuco, Recife, Brasil. 1995.

MOURA, A. R. L. U (1999). Análise Tridimensional de Interação Solo - Estrutura em Edifícios. Solos e Rochas, v.22, n.2, p.87-100.

MYLONAKIS, G.; GAZETAS, G (1998). Settlement and additional internal forces of grouped piles in layered soil. Géotechnique, London, England, v.48, n.1, p.5572 . 
NAKAGUMA, R. K (1979). Three-dimensional elastostatics using the boundary element method. Tese (Doutorado) - University of Southampton, New York, USA. 1979.

NBR6118. Projeto e execução de obras de concreto armado. Associação Brasileira de Normas Técnicas.

NBR6123. Forças devidas ao vento em edificações. Associação Brasileira de Normas Técnicas.

OÑATE, E. I. N (1995). Calculo de estructuras por el metodo de elementos finitos. Barcelona, Espanha: Centro Internacional de Métodos Numéricos en Engeniería.

OSHIMA, S. T (2004). Uma combinação MEC/MEF para análise da interação de estacas inclinadas e o solo. 84 p. Dissertação (Mestrado) - Escola de Engenharia de São Carlos, Universidade de São Paulo, São Carlos, Brasil. 2004.

OTTAVIANI, M (1975). Three-dimensional finite element analysis of vertically loaded pile groups. Géotechnique, London, England, v.25, n.2, p.159-174.

PACCOLA, R. R (2004). Análise não linear física de placas e cascas anisotrópicas laminadas acopladas ou não com meio contínuo tridimensional viscoelástico através da combinação entre o MEC e o MEF. 192p. Tese (Doutorado) - Escola de Engenharia de São Carlos, Universidade de São Paulo, São Carlos, Brasil. 2004.

PAN, E (1997). Static Green's functions in multilayered half spaces. Applied Math. Modelling, New York, v.21, p.509-521.

PELETEIRO, S. C (1996). Utilização da formulação livre para desenvolvimento de um elemento de membrana com liberdades rotacionais. Dissertação (Mestrado) - Escola de Engenharia de São Carlos, Universidade de São Paulo, São Carlos, Brasil. 1996.

POULOS, H. G (1967). Stresses and displacements in an elastic layer underlain by rough rigid base. Géotechnique, London, England, v.17, p.378-410.

POULOS, H. G (1968). Analysis of the settlement of pile groups. Géotechnique, London, England, v.18, p.449-471.

POULOS, H. G. Behaviour of laterally loaded piles: 1 - single piles. Journal of the Soil Mechanics and Foundations Division, [S.1.], v.97, n.5, p.711-731, 1971.

POULOS, H. G. Behaviour of laterally loaded piles: 2 - single piles. Journal of the Soil Mechanics and Foundations Division, [S.1.], v.97, n.5, p.733-751, 1971. 
POULOS, H. G (1971). Behaviour of laterally loaded piles: 1 - single piles. Journal of the Soil Mechanics and Foundations Division, v.97, n.5, p.711-731.

POULOS, H. G (1971). Behaviour of laterally loaded piles: 2 - single piles. Journal of the Soil Mechanics and Foundations Division, v.97, n.5, p.733-751.

POULOS, H. G.; DAVIES, H. G (1968). The settlement behaviour of single axially loaded incompressible piles and piers. Géotechnique, London, England, v.18, p.351-371.

RANDOLPH, M. F.; WROTH, C. P (1979). An analysis of the vertical deformation of pile groups. Géotechnique, London, England, v.29, p.423-439.

REIS, J. H. C (2000). Interação solo-estrutura de grupos de edifícios com fundações superficiais em argila mole. 148p. Dissertação (Mestrado) - Escola de Engenharia de São Carlos, Universidade de São Paulo, São Carlos, Brasil. 2000.

RIBEIRO, D. B.; ALMEIDA, V. S.; PAIVA, J. B (2004). Uma formulação alternativa para analisar a interação solo não-homogêneo/ fundação/ superestrutura via acoplamento MEC-MEF. In: XXXI JORNADAS SUD-AMERICANAS DE INGENIERÍA ESTRUCTURAL, 2004, Mendoza, Argentina. Anais... CD-ROM.

RIOS, B. M. C (1991). Análise tridimensional e envoltória de esforços em edifícios altos sujeitos aações verticais e laterais. 247p. Dissertação (Mestrado) - Escola de Engenharia de São Carlos, Universidade de São Paulo, São Carlos, Brasil. 1991.

SADECKA, L (2000). A finite/infinite element analysis of thick plate on a layered foundation. Computers and Structures, England, v.76, p.603-610.

SILVA, M. T (1994). Interação solo-estrutura de fundação em estacas de solocimento e concreto. 101p. Dissertação (Mestrado) - UnB, Brasília, Brasil. 1994.

SMALL, J. C.; BOOKER, J. R (1984). Finite layer analysis of layered elastic materials using a flexibility approach. Part 1 - Strip loadings. International Journal for Numerical Methods in Engineering, England, v.20, p.1025-1037.

SOUTHCOTT, P. H.; SMALL, J. C (1996). Finite layer analysis of vertically loaded piles and pile groups. Computer and Geotechnics, England, v.18, n.1, p.47-63.

STEINBRENNER, W (1934). Tafeln zur Setzungberechnung. Strasse, v.1, p.221.

TA, L. D.; SMALL, J. C (1998). Analysis and performance of piled raft foundations on layered soils-case studies. Soil and Foundations, v.38, n.4, p.145-150. 
VEIGA, J (2000). Análise de problemas tridimensionais solo-estrutura pelo método dos elementos finitos no domínio de Fourier. 85p. Dissertação (Mestrado) - Pontifícia Universidade Católica do Rio de Janeiro, Rio de Janeiro, Brasil. 2000.

WANG, C. M.; IANG, Y. X.; WANG, Q (2001). Axisymmetric buckling of reddy circular plates on Pasternak foundation. Journal Engineering Mechanic, v.127, p.254-259.

WINKLER, E (1867). Die Lehre von Elastizität und Festigkeit. Prague, p.182.

WITT, M (1984). Solutions of plates on a heterogeneous elastic foundation. Computers and Structures, England, v.18, p.41-45. 


\section{Apêndice A}

\section{Integral singular}

O objetivo deste apêndice é expor as passagens matemáticas implícitas entre as igualdades 2.77 e 2.79, que são parte do processo dedutivo apresentado no capítulo 2 .

As hipóteses iniciais são contorno suave no ponto $i$ e uma semi-esfera de raio $\varepsilon$ envolvendo este ponto, conforme mostrado na figura 2.6. A expressão a ser analisada é a seguinte:

$$
\int_{\Gamma} p_{l k}^{*} \cdot u_{k} \cdot d \Gamma=\lim _{\varepsilon \rightarrow 0}\left\{\int_{\Gamma-\Gamma_{\varepsilon}} p_{l k}^{*} \cdot u_{k} \cdot d \Gamma\right\}+\lim _{\varepsilon \rightarrow 0}\left\{\int_{\Gamma_{\varepsilon}} p_{l k}^{*} \cdot u_{k} \cdot d \Gamma\right\}
$$

Considerando somente a integral em $\Gamma_{\varepsilon}$, tem-se que:

$$
I=\lim _{\varepsilon \rightarrow 0}\left\{\int_{\Gamma_{\varepsilon}} u_{k} \cdot p_{l k}^{*} \cdot d \Gamma\right\}=u_{k}^{i} \cdot \lim _{\varepsilon \rightarrow 0}\left\{\int_{\Gamma_{\varepsilon}} p_{l k}^{*} \cdot d \Gamma\right\}
$$

Substituindo a expressão 2.52 na equação A.2, obtém-se:

$$
\begin{gathered}
I=\lim _{\varepsilon \rightarrow 0}\left\{\int _ { \Gamma _ { \varepsilon } } u _ { k } \cdot ( \frac { - 1 } { 8 \cdot \pi \cdot ( 1 - \nu ) \cdot r ^ { 2 } } ) \cdot \left[\frac{\partial r}{\partial \eta} \cdot\left[(1-2 \cdot \nu) \cdot \delta_{l k}+3 \cdot \frac{\partial r}{\partial x_{l}} \cdot \frac{\partial r}{\partial x_{k}}\right]+\right.\right. \\
\left.\left.+(1-2 \cdot \nu) \cdot\left(\frac{\partial r}{\partial x_{l}} \cdot \eta_{k}-\frac{\partial r}{\partial x_{k}} \cdot \eta_{l}\right)\right] \cdot d \Gamma\right\}
\end{gathered}
$$

Como a integral está sendo feita no controno $\Gamma_{\varepsilon}$ a partir do ponto $i$, que se encontra no centro da semi-esfera, conclui-se que o raio $\varepsilon$ da esfera é igual à distância $r$ entre o ponto campo e o ponto fonte. Com isto, torna-se mais prático adotar um sistema de coordenadas esférico com centro no ponto $i$. Assim, tornam-se válidas as igualdades:

$$
\eta_{k}=\frac{\partial r}{\partial x_{k}}
$$


e

$$
\eta_{l}=\frac{\partial r}{\partial x_{l}}
$$

Portanto:

$$
\frac{\partial r}{\partial x_{l}} \cdot \eta_{k}-\frac{\partial r}{\partial x_{k}} \cdot \eta_{l}=\frac{\partial r}{\partial x_{l}} \cdot \frac{\partial r}{\partial x_{k}}-\frac{\partial r}{\partial x_{k}} \cdot \frac{\partial r}{\partial x_{l}}=0
$$

Assim, a expressão A.6 se reduz a:

$$
I=\lim _{\varepsilon \rightarrow 0}\left\{\int_{\Gamma_{\varepsilon}} u_{k} \cdot\left(\frac{-1}{8 \cdot \pi \cdot(1-\nu) \cdot r^{2}}\right) \cdot\left[\frac{\partial r}{\partial \eta} \cdot\left[(1-2 \cdot \nu) \cdot \delta_{l k}+3 \cdot \frac{\partial r}{\partial x_{l}} \cdot \frac{\partial r}{\partial x_{k}}\right]\right] \cdot d \Gamma\right\}
$$

Como se trata de uma semi-esfera, o termo $\frac{\partial r}{\partial \eta}$ é igual a um, pois a geometria da semi-esfera garante que a normal à sua superfície sempre esteja alinhada com seu raio. A próxima passagem utiliza propriedades do sistema de coordenadas esférico, ilustrado na figura A.1.

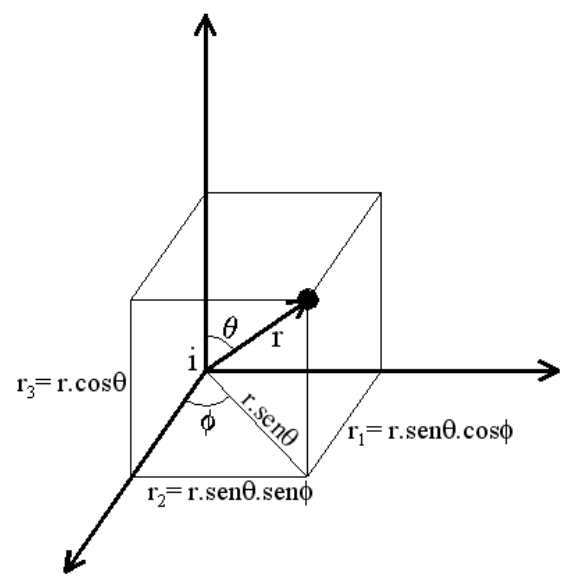

Figura A.1: Sistema de coordenadas esféricas

Fazendo o índice $l$ igual a um e considerando o sistema de coordenadas esférico, pode-se reescrever a expressão A.7 como:

$$
\begin{gathered}
I=\lim _{\varepsilon \rightarrow 0}\left\{-\int_{\Gamma_{\varepsilon}}\left\{u_{1}^{i} \cdot(1-2 \cdot \varepsilon)+3 \cdot u_{1}^{i} \cdot e_{1} \cdot e_{1}+3 \cdot u_{2}^{i} \cdot e_{1} \cdot e_{2}+3 \cdot u_{3}^{i} \cdot e_{1} \cdot e_{3}\right\} \cdot\right. \\
\left.\frac{\operatorname{sen} \theta \cdot d \theta \cdot d \phi}{8 \cdot \pi \cdot(1-\nu)}\right\}
\end{gathered}
$$

onde os termos $e_{i}$ são versores na direção $i$, tal que:

$$
e_{i}=\eta_{i}=\frac{\partial r}{\partial x_{i}}
$$

Após efetuar a integral de A.9 chega-se à seguinte expressão:

$$
I=\left(\frac{-1}{8 \cdot \pi \cdot(1-\nu) \cdot r^{2}}\right) \cdot[(1-2 \cdot \nu) \cdot 2 \cdot \pi+2 \cdot \pi] \cdot u_{1}^{i}
$$




$$
\begin{gathered}
I=\frac{-4 \cdot(1-\nu)}{8 \cdot(1-\nu)} \cdot u_{1}^{i} \\
I=-\frac{1}{2} \cdot u_{1}^{i}
\end{gathered}
$$

Pode-se constatar que o mesmo ocorre quando se considera $l$ igual a dois ou $l$ igual a três. Assim, conclui-se com a expressão geral:

$$
\lim _{\varepsilon \rightarrow 0}\left\{\int_{\Gamma_{\varepsilon}} u_{k} \cdot p_{l k}^{*} \cdot d \Gamma\right\}=-\frac{1}{2} \cdot u_{l}^{i}
$$

A partir da expressão A.13, torna-se possível chegar à expressão final para a equação de contorno em um ponto onde $\Gamma$ é suave. Esta é:

$$
\frac{1}{2} \cdot u_{l}^{i}+\int_{\Gamma} u_{k} \cdot p_{l k}^{*} \cdot d \Gamma=\int_{\Gamma} p_{k} \cdot u_{l k}^{*} \cdot d \Gamma=\int_{\Omega} b_{k} \cdot u_{l k}^{*} \cdot d \Omega
$$

